\title{
Verticillium dahliae transcription factors Som1 and Vta3 control microsclerotia formation and sequential steps of plant root penetration and colonisation to induce disease
}

\author{
Dissertation \\ for the award of the degree \\ "Doctor rerum naturalium" \\ of the Georg-August Universität Göttingen \\ within the doctoral program Biology \\ of the Georg-August University School of Science
}

submitted by

Thuc Tri Bui

from Thai Nguyen, Vietnam

Göttingen 2017 


\section{Thesis Committee}

Prof. Dr. Gerhard H. Braus

Department of Molecular Microbiology and Genetics Institute of Microbiology and Genetics

Georg-August Universität Göttingen

\section{Prof. Dr. Stefanie Pöggeler}

Department of Genetics of Eukaryotic Microorganisms Institute of Microbiology and Genetics

Georg-August Universität Göttingen

\section{Members of the Examination Board}

Reviewer I

Prof. Dr. Gerhard H. Braus

Department of Molecular Microbiology and Genetics Institute of Microbiology and Genetics

Georg-August Universität Göttingen

Reviewer II

\section{Prof. Dr. Stefanie Pöggeler}

Department of Genetics of Eukaryotic Microorganisms Institute of Microbiology and Genetics

Georg-August Universität Göttingen

\section{Further members of the Examination Board}

Prof. Dr. Ivo Feussner

Department of Biochemistry of the Plant

Albrecht-von-Haller-Institute of Plant Sciences

Prof. Dr. Kai Heimel

Department of Molecular Microbiology and Genetics Institute of Microbiology and Genetics

\section{PD Dr. Michael Hoppert}

Department General Microbiology Institute of Microbiology and Genetics

\section{Prof. Dr. Rolf Daniel}

Department of Genomic and Applied Microbiology Institute of Microbiology and Genetics 


\section{Affirmation}

I hereby declare that this thesis was written independently and with no other sources and aids than quoted

Göttingen, 3.10.2017

Thuc Tri Bui 
This work was accomplished in the group of Prof. Dr. Gerhard H. Braus, at the Department of Molecular Microbiology and Genetics at the Institute of Microbiology and Genetics, Georg-August Universität Göttingen.

Parts of my work will be published in:

Tri-Thuc Bui, Rebekka Harting, Susanna A. Braus-Stromeyer, Van-Tuan Tran, Oliver Valerius, Rabea Schlüter, Claire E. Stanley, Alinne Ambrósio, Gerhard H. Braus (2017). Verticillium dahliae transcription factors Som1 and Vta3 control microsclerotia formation and sequential steps of plant root penetration and colonisation to induce disease. Submitted for publication. 


\section{Table of contents}

Summary 1

Zusammenfassung 2

1. Introduction 3

$1.1 \quad$ Verticillium dahliae - a pathogen of wilt diseases 3

1.1.1 V. dahliae is a threatening plant pathogenic fungus 3

1.1.2 Verticillium morphology 5

1.1.3 Disease symptoms of $V$. dahliae on tomatoes 7

$1.1 .4 \quad$ V. dahliae disease cycle 8

1.2 Adhesion is essential for fungal pathogens 9

1.2.1 Adhesion in yeasts 10

1.2.2 Adhesion and virulence in filamentous fungi 13

1.2.3 Adhesion and virulence in V. dahliae 16

1.2.4 Wing helix-turn-helix DNA binding proteins 19

1.3 Regulation of conidia and microsclerotia formation 20

1.3.1 Regulation of conidation 20

1.3.2 Regulation of microsclerotia formation 21

$\begin{array}{lll}1.4 & \text { Aim of this work } & 22\end{array}$

$2 \quad$ Materials and Methods $\quad 24$

$2.1 \quad$ Materials $\quad 24$

2.1.1 Chemicals 24

2.1.2 Primers 24

2.1.3 Plasmids 29

2.1.4 Organisms 30

2.1.4.1 Bacterial strains and their cultivation 30

2.1.4.2 Fungal strains and their cultivation 30

2.2 Methods 33

2.2.1 Bioinformatic analysis 33

2.2.2 Gene deletion, complementation, and overexpression 33

2.2.2.1 Gene deletion 33

2.2.2.2 Gene complementation 35

2.2.2.3 Gene overexpression 36

$\begin{array}{lll}2.2 .3 & \text { Genetic manipulations } & 37\end{array}$ 
2.2.3.2 A. tumefaciens transformation 38

2.2.3.3 S. cerevisiae transformation 38

2.2.3.4 V. dahliae transformation 39

2.2.4 Confirmation of transformation 39

2.2.4.1 DNA purification 39

2.2.4.2 PCR amplification $\quad 41$

2.2.4.3 Southern hybridization 42

2.2.5 Phenotypical analyses 42

2.2.5.1 Microsclerotia counting 42

2.2.5.2 Conidia examination 42

2.2.5.3 Hyphal branching test 43

2.2.5.4 Localisation study 43

2.2.5.5 Oxidative stress test 43

2.2.5.6 Adhesion examination 44

2.2.6 Plant infection test 44

2.2.6.1 Tomato infection study 44

2.2.6.2 Arabidopsis root infection test 45

2.2.6.3 Scan electron microscopy $\quad 45$

2.2.7 Protein methods 46

2.2.7.1 Protein isolation 46

2.2.7.2 Proteomic analysis 46

2.2.7.3 Western hybridization $\quad 47$

2.2.7.4 GFP trap assay $\quad 47$

$\begin{array}{lll}2.2 .8 & \text { Gene expression quantification } & 47\end{array}$

3 Results 49

3.1 The transcription factors SOM1 and VTA3 can reprogram $\begin{array}{ll}\text { non-adhesive yeast strain } & 49\end{array}$

3.1.1 SOM1 and VTA3 genes encode proteins comprising a LisH or a wing helix-turn-helix DNA binding domain 49

3.1.2 Som1 and Vta3 are nuclear proteins 51

3.1.3 Som1 and Vta3 can rescue adhesion of FLO8-defective S. cerevisiae strains 
3.1.4 Low expression of SOM1 can activate flocculation genes

3.1.5 Activation of VTA3 can stimulate expression of flocculation genes

3.2 Transcription factors SOM1 and VTA3 are required for morphology and virulence in V. dahliae

3.2.1 Deletion and complementation of SOM1 and VTA3 in V. dahliae 56

3.2.2 Som1 promotes adhesion in $V$. dahliae 58

3.2.2.1 Som1 is necessary for hyphal clumping and suppresses biomass formation

3.2.2.2 Som1 is needed for adherence on abiotic surfaces

3.2.3 Som 1 and $\mathrm{Vta} 3$ are required for pathogenicity

3.2.3.1 Som1 and Vta3 are involved in fungal pathogenicity

3.2.3.2 Fungal Som1 and Vta3 are required for sequential steps of plant root penetration and colonisation

3.2.4 Som1 and Vta3 support conidia and microsclerotia formation

3.2.4.1 Som1 and Vta3 promote conidia formation

3.2.4.2 Som1 and Vta3 control microsclerotia formation

3.2.5 Som 1 and $V t a 3$ antagonise in oxidative stress response

3.2.6 Som1 and Vta3 are needed for hyphal growth of $V$. dahliae on agar plates

3.2.7 Som1 is essential for hyphal development in $V$. dahliae

3.2.8 Som1 and VTA3 regulate the expression of VTA genes and related adhesion, conidia and microsclerotia formation, and virulence genes

3.2.8.1 Som1 and Vta3 regulate the expression of VTA genes

3.2.8.2 Som1 control expression of genes involved in adhesion

3.2.8.3 Som1 and Vta3 control expression of genes involved in conidia and microsclerotia formation, oxidative stress response and virulence

3.2.8.4 Som1 interacts with protein Ptab while Vta3 interacts with the transcriptional co-repressor Ssn6

\subsection{A. fumigatus SOMA can rescue the deletion of SOM1 in V. dahliae 86}

\begin{tabular}{lll}
\hline 4. & Discussion & 89
\end{tabular}

4.1 The transcription factors Som1 and Vta3 support adhesion of S. cerevisiae

4.1.1 Som1 presumably binds to promoter regions of flocculation genes in S. cerevisiae for activation 
4.1.2 Vta3 might activate adhesion through repressing the negatively acting SFL1 in S. cerevisiae

4.2 The Transcription factors Som1 and Vta3 promote fungal development and virulence

4.2.1 Som1 and Vta3 control transcription factors for adhesion

4.2.2 Som 1 controls adhesion and penetration in $V$. dahliae

4.2.3 Som1 and Vta3 promote pathogenicity

4.2.4 Som1 and Vta3 are essential for conidia and microsclerotia formation 96

4.2.5 Som1 and Vta3 antagonise the oxidative stress response

4.2.6 Som1 and Vta3 are required for hyphal development

4.3 AfSom1 and VdSom1 fulfil similar functions in plant and human pathogens

4.4 Outlook

References

Abbreviations

List of Figures

List of Tables 125

Acknowledgements 126

Curriculum vitae 


\section{Summary}

Verticillium dahliae belongs to the soil-borne ascomycete fungi. It causes wilt diseases and early senescence in more than 200 plant species including economically important crops. It can exist in the soil without a host for a decade by forming microsclerotia. Root exudates induce germination of microsclerotia. $V$. dahliae enters its hosts through root infection, colonises the root cortex and invades the xylem vessels. The host infection of pathogenic fungi requires penetration and colonisation processes. The penetration of the root surface needs adhesive proteins at several stages during the host-parasite interaction. Adhesion proteins are not well described in $V$. dahliae whereas they are well studied in Saccharomyces cerevisiae. S. cerevisiae Flo8 is a transcription factor of adhesion, which regulates the expression of flocculation genes such as FLO1 and FLO11. The defective FLO8 strain is unable to adhere to agar plates or to flocculate in liquid medium. $V$. dahliae nuclear transcription factors Som1 and Vta3 can rescue adhesion in a FLO8-deficient $S$. cerevisiae strain. Som1 and Vta3 induce the expression of FLO1 and FLO11 genes encoding adhesins. The SOM1 and VTA3 genes were deleted and their function in fungal induced plant pathogenesis was studied by genetic, cell biological, proteomic and plant pathogenicity experiments. $V$. dahliae Som1 and Vta3 are sequentially required for root penetration and colonisation of the plant host. Som1 supports fungal adhesion and root penetration and is required earlier than Vta3 in the colonisation of plant root surfaces and tomato plant infection. Som1 controls septa positioning, the size of vacuoles, and subsequently hyphal development including aerial hyphae formation and normal hyphal branching. Som1 and Vta3 control conidia and microsclerotia formation and antagonise in oxidative stress response. The molecular function of Som1 is conserved between the plant pathogen $V$. dahliae and the opportunistic human pathogen Aspergillus fumigatus. Som1 controls the expression of genes for adhesion and oxidative stress response. Som1, as well as Vta3, regulate a genetic network for conidia and microsclerotia formation and pathogenicity of $V$. dahliae. 


\section{Zusammenfassung}

Verticillium dahliae gehört zu den bodenbürtigen Askomyceten. Dieser Pilz verursacht Welke-Erkrankungen und verfrühtes Altern in mehr als 200 verschiedenen, auch ökonomisch wichtigen Pflanzen. Verticillium kann im Boden ohne Wirtspflanze durch die Bildung von Mikrosklerotien bis zu 10 Jahre überleben. Wurzelexsudate induzieren die Auskeimung der Mikrosklerotien. $V$. dahliae infiziert seinen Wirt durch die Wurzel, besiedelt den Wurzelkortex und dringt dann in die Xylemgefäße ein. Die Infektion des Wirts durch pathogene Pilze erfordert Penetrations- und Kolonisierungsprozesse. Am Eindringen durch die Wurzeloberfläche sind adhäsive Proteine an verschiedenen Stellen der WirtParasit-Interaktion beteiligt. Adhäsive Proteine sind in S. cerevisiae gut untersucht, während nur wenig über sie in $V$. dahliae bekannt ist. Der AdhäsionsTranskriptionsfaktor Flo8 aus Hefe reguliert die Expression der sogenannten „Flocculation"-Gene wie zum Beispiel FLO1 und FLO11. Ein Stamm ohne FLO8 ist nicht in der Lage an Agarmedium zu haften und in Flüssigmedium auszuflocken. Die im Zellkern lokalisierten Transkriptionsfaktoren Som1 und Vta3 können die Adhäsion in einem S. cerevisiae Stamm, welchem FLO8 fehlt, wiederherstellen. Som1 und Vta3 induzieren die Expression von FLO1 und FLO11, welche Adhäsine kodieren. Die SOM1 und VTA3 Gene wurden deletiert und ihre Funktion in der durch Pilze verursachten Pflanzenpathogenese wurde durch genetische, zellbiologische, Proteom- und Pflanzenpathogenitätsexperimente untersucht. V. dahliae Som1 und Vta3 sind sequenziell für die Penetration und Kolonisation des Pflanzenwirts erforderlich. Som1 unterstützt die pilzliche Adhäsion sowie das Eindringen in die Wurzel. Somit wird es früher für die Besiedlung der Pflanzenwurzeloberfläche und die Tomateninfektion benötigt als Vta3. Som1 kontrolliert darüber hinaus die Positionierung von Septen und die Größe von Vakuolen und folglich auch die Entwicklung von Hyphen inklusive der Bildung von Lufthyphen und normalen Hyphenverzweigungen. Som1 und Vta3 beeinflussen die Bildung von Konidien und Mikrosklerotien und wirken sich in der Antwort auf oxidativen Stress entgegen. Die molekulare Funktion von Som1 ist zwischen dem Pflanzenpathogen V. dahliae und dem opportunistischen Humanpathogen Aspergillus fumigatus konserviert. Som1 kontrolliert die Expression von Genen welche für Adhäsion und die Antwort auf oxidativen Stress benötigt werden. Sowohl Som1 als auch Vta3 regulieren ein genetisches Netzwerk für die Bildung von Konidien und Mikrosklerotien sowie die Pathogenität von V. dahliae. 


\section{Introduction}

\subsection{Verticillium dahliae - a pathogen of wilt diseases}

Verticillium species are soil-borne plant pathogenic fungi which cause high losses of crops (Pegg \& Brady, 2002; Berlanger \& Powelson, 2005). The name Verticillium was given because of the phialide arrangement in verticillate shape around the conidiophores (Pegg \& Brady, 2002; Berlanger \& Powelson, 2005). There are three different species of Verticillium including Verticillium albo-atrum, Verticillium dahliae, and Verticillium longisporum (Pegg \& Brady, 2002). However, the first Verticillium strain was detected in 1879 (Reinke \& Berthold, 1879) until 1913 V. dahliae which causes wilt on dahlia (Asteraceae family) was first described (Isaac, 1947).

\subsubsection{V. dahliae is a threatening plant pathogenic fungus}

V. dahliae strains cause wilting diseases and early senescence in more than 200 plant species of economically important crops including tomato, potato, cotton, cabbages, and strawberries (Pegg \& Brady, 2002). This species is causing significant loss in crop yield and are widely spread.

A $V$. dahliae strain can enter the root and develop resting structures not only in more than 200 plant host species but also in non-host plants (Pegg \& Brady, 2002; Berlanger \& Powelson, 2005). After entering the plant roots, this fungus produces conidia which are transported in the transpiration stream to any part of the plant. In which, conidia germinate and colonise the plant (Pegg \& Brady, 2002; Berlanger \& Powelson, 2005). V. dahliae produces resting structures which are called microsclerotia to survive in the soil without host plants. Microsclerotia are formed in dry tissues when the plant dies. Thick cell walls with dense melanin deposits of microsclerotia protect the fungus against extreme temperatures, enzymatic lysis, and UV light. They still can recover from animal feces after staying two days in the stomach (Pegg \& Brady, 2002; Berlanger \& Powelson, 2005). Therefore, microsclerotia can survive without hosts for a decade in the soil (Pegg \& Brady, 2002). Additionally, V. dahliae can enter non-host plants and produce microsclerotia, which cause no symptoms or diseases (Pegg \& Brady, 2002; 
Berlanger \& Powelson, 2005). Because of these reasons, it is not easy to treat $V$. dahliae even when using crop rotations or fungicides (Pegg \& Brady, 2002). Therefore, $V$. dahliae causes a loss of billions of dollars in annual crops worldwide (Pegg \& Brady, 2002; Berlanger \& Powelson, 2005).

Conidia and microsclerotia of $V$. dahliae are easily transported worldwide by different ways. For instance, conidia are tiny cells which can be transported through xylem vessel systems with the transpiration stream from the root vessels into the shoot and thereby distributing the fungus to the whole plant. Therefore it is easy transported with crop products (Pegg \& Brady, 2002; Berlanger \& Powelson, 2005; Inderbitzin et al., 2011b). Furthermore, they are easily transported by air for 20 feet or by water stream such as rivers and irrigation canals when the water was re-used. $V$. dahliae may also be distributed by contaminated seeds, insects, vegetative cutting, transplant, hand tools or farm machinery (Pegg \& Brady, 2002; Berlanger \& Powelson, 2005). Microsclerotia can also be found in seeds of infected plants, therefore, they are easily transported worldwide when crop products are exported or imported (Pegg \& Brady, 2002). Nowadays, this fungus can be found worldwide in countries with cool or warm climate (Figure 1).

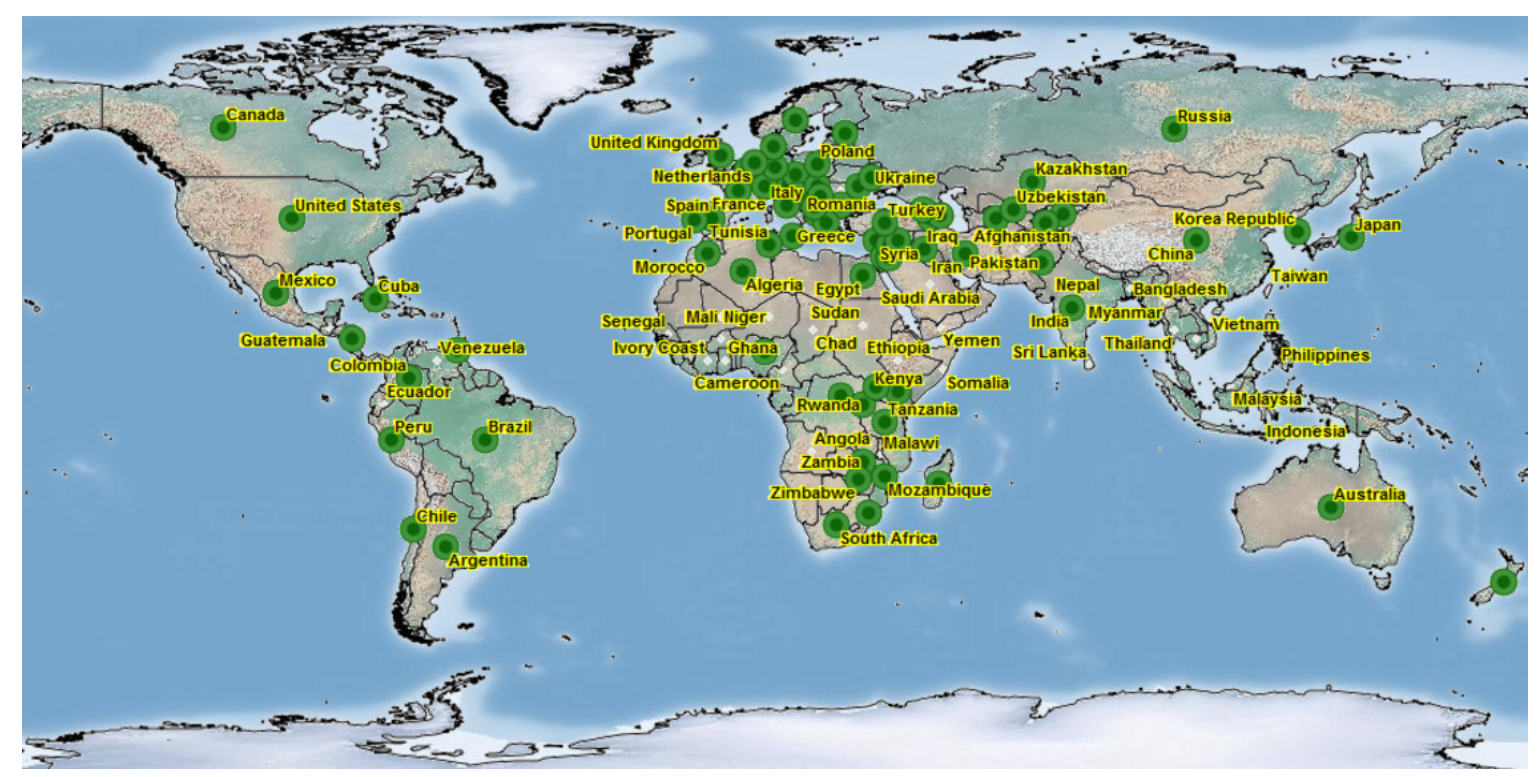

Figure 1. V. dahliae distribution. $V$. dahliae species were found worldwide from cool to warm climate. They are more common in Europe, America, and Africa than in Asia and Australia. Figure taken from http://www.plantwise.org/KnowledgeBank/Datasheet. aspx?dsid=56275. 


\subsubsection{Verticillium morphology}

Hyphae of $V$. dahliae are mostly haploid, but hyphal tips may be multinucleate. They are hyaline, simple or branched, septated and multinucleated. Hyphal septa are perforated, but nuclei have not been reported to traverse the pore (Pegg \& Brady, 2002; Berlanger \& Powelson, 2005). The hyphal extension is directly proportional to the availability of growth capabilities. Diffusible morphogenic factors in $V$. dahliae inhibit hyphal elongation and conidiation and induce lateral branching (Brandt, 1967). Conidia are single cells which are born on phialides (Figure 2a, b, c). These phialides are arranged in whorls around conidiophores which are branched aerial hyphae(Pegg \& Brady, 2002; Berlanger \& Powelson, 2005). Each phialide carries a mass of conidia which are named conidiospore cluster in the following (Figure 2c). Conidia are hyaline and ovoid to an elongated shape. They have thin cell walls without melanin deposits (Figure $2 \mathrm{~d}$ ). Conidia are very small (3.5-5.5 $\mu \mathrm{m}$ ) and are transported easily with the transpiration stream in plants (Pegg \& Brady, 2002). Fungal materials such as microsclerotia can be therefore found in whole plants including seeds.

The resting structures, which are formed by hyphal welting, are usually found in the dead tissue of infected plants. They have thick cell walls with dense melanin layers. Black microsclerotia are found in the dead plant as black dots (Figure $2 \mathrm{e}, \mathrm{f}, \mathrm{g}$ ) (Pegg \& Brady, 2002; Berlanger \& Powelson, 2005; Inderbitzin et al., 2011b). These structures help $V$. dahliae to survive in the dead plants and the soil up to 15 years or after going through the animal stomach (Pegg \& Brady, 2002). 


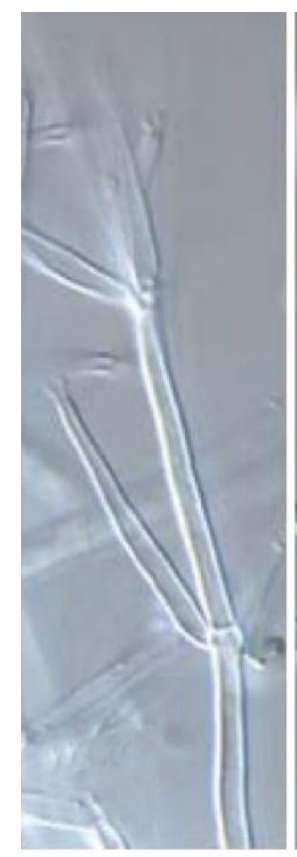

(a)

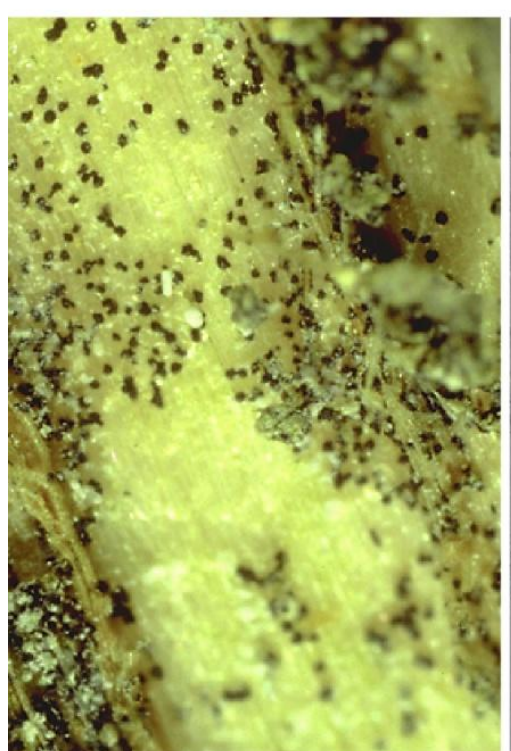

(e)

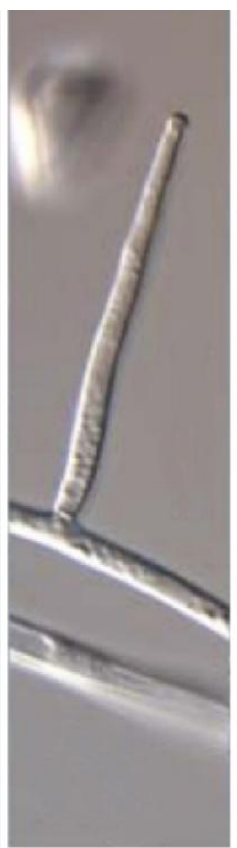

(b)

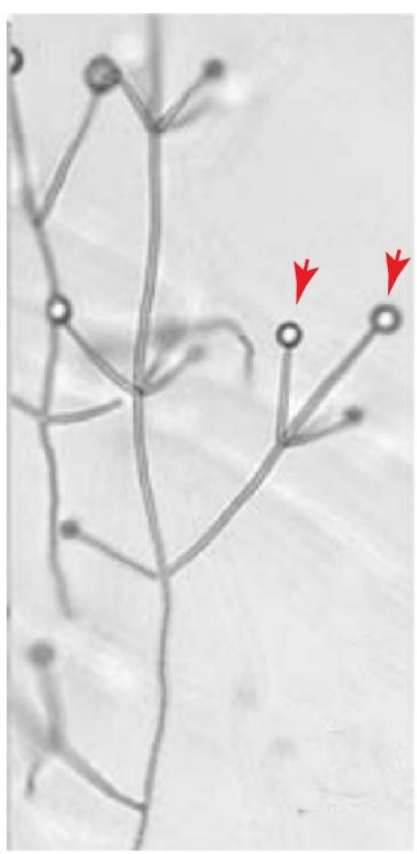

(c)

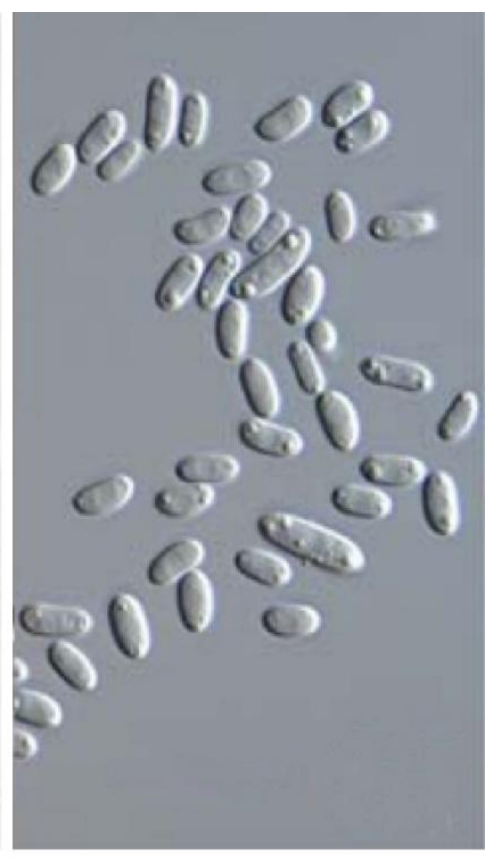

(d)

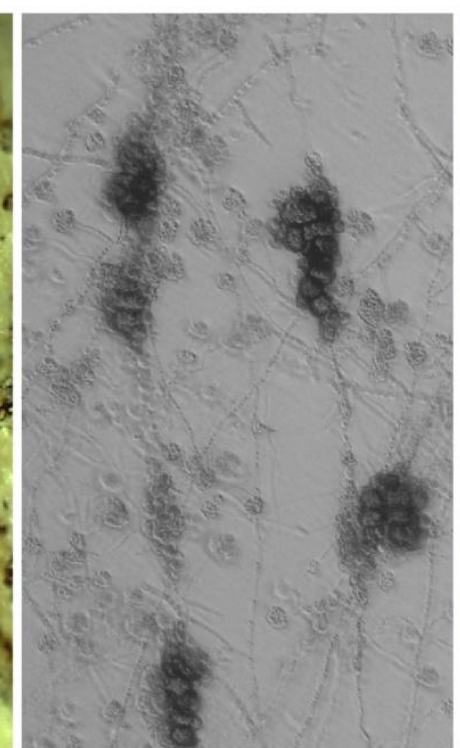

(f)

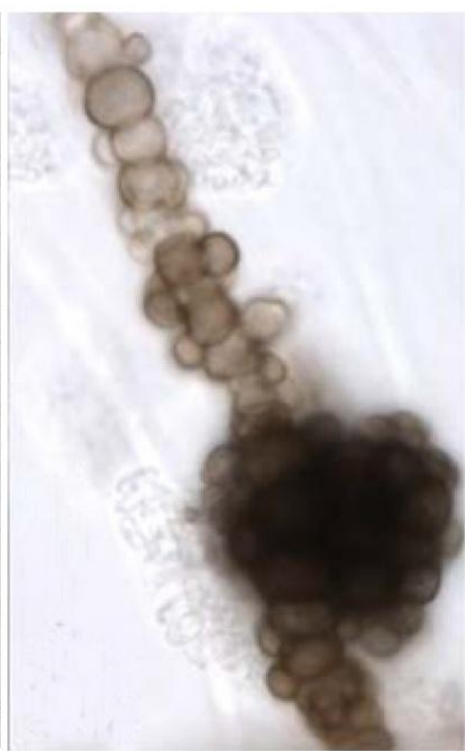

(g)

Figure 2. Conidia and microsclerotia of $\boldsymbol{V}$. dahliae. (a) Whorl phialide. (b) Solitary phialide. (c) Branched conidiophore. (d) Conidia. (e) Microsclerotia in planta. (f) Microsclerotia on agar plates. (g) The structure of a single microsclerotium. The arrowhead shows a conidiospore cluster. This figure is modified from Inderbitzin et al. (2011a). 


\subsubsection{Disease symptoms of $V$. dahliae on tomatoes}

Disease symptoms caused by $V$. dahliae are quite similar to Fusarium wilt symptoms which are difficult to distinguish in the field (Pegg \& Brady, 2002; Berlanger \& Powelson, 2005). Tomato infected stems have vascular discoloration consisting of dark-colored, elongated, necrotic tissue. The vascular discoloration may be accompanied by external symptoms such as wilting, yellowing, slow growth, abnormally heavy fruits or seeds, and death of leaves and plants (Pegg \& Brady, 2002; Berlanger \& Powelson, 2005). The lower and older leaves turn yellow earlier, wilt and dry later on. Infected plants are usually wilted in the midday and recovered in the evening. Most of the infected plants are stunted (Figure 3). Disease symptoms are more pronounced when plants are under drought stress (Pegg \& Brady, 2002; Berlanger \& Powelson, 2005).

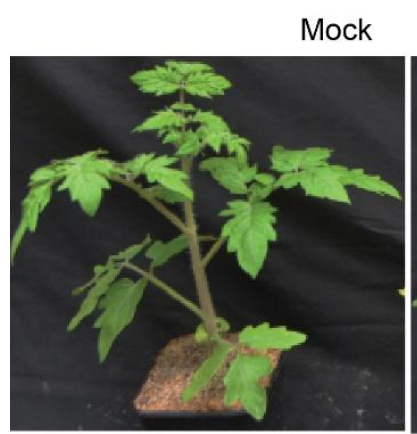

(a)

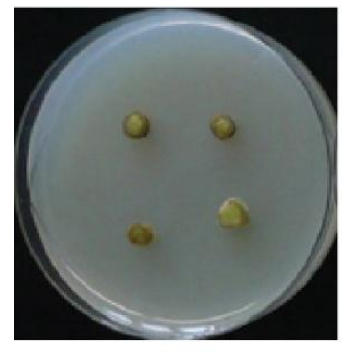

(c)

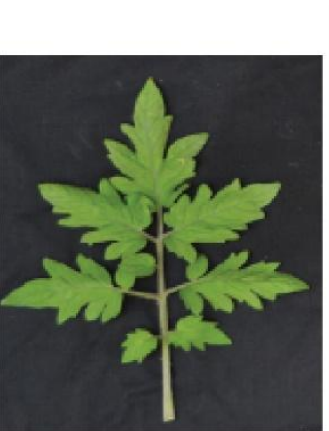

(b)

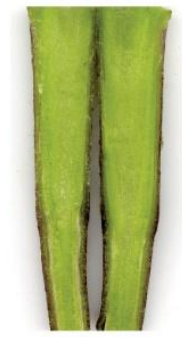

(d)

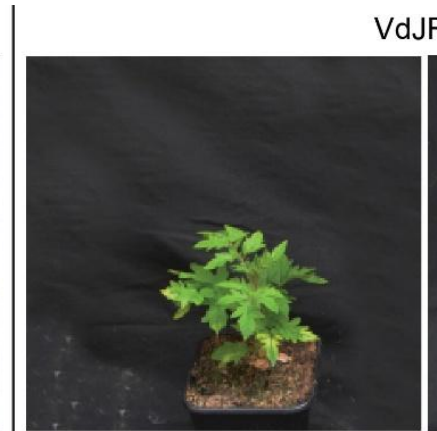

(a)

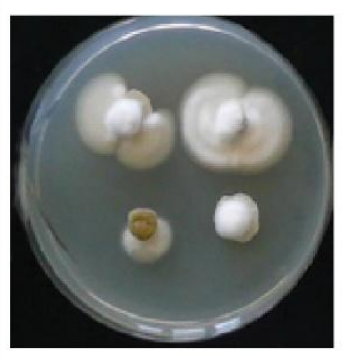

(c)
VdJR2

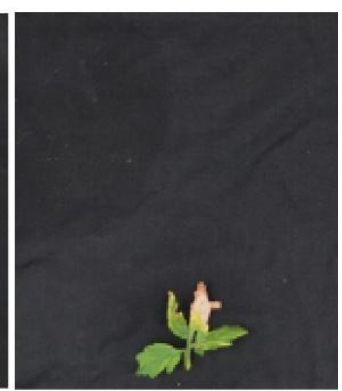

(b)

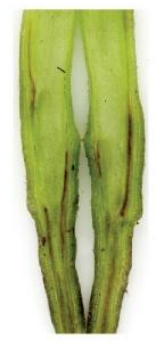

(d)

Figure 3. Wilt disease symptoms of $\boldsymbol{V}$. dahliae. $V$. dahliae causes wilt diseases and early senescence in tomato plants (a) Height of tomato plants. (b) Length and disease symptoms in the first leaves. (c) Outgrowth of fungi from stems. (d) Disease symptoms in hypocotyls. Figure is modified from Tran et al. (2014). 


\subsubsection{V. dahliae disease cycle}

The life cycle of $V$. dahliae is monocyclic. It has only one disease cycle as in other Verticillium species (Figure 4). The life cycle of $V$. dahliae can be divided into 2 steps root entering and developing in the plant. The root entering starts when microsclerotia are stimulated by root exudates or nutrients to germinate and grow in the soil toward the root surface (Berlanger \& Powelson, 2005; Eynck et al., 2007). The mycelium can reach up to one $\mathrm{cm}$ into the soil without nutrient supply before attaching to the plant root surface. Hyphae can infect and colonise root tips or follow root hairs to the root surface for penetration (Pegg \& Brady, 2002; Berlanger \& Powelson, 2005).

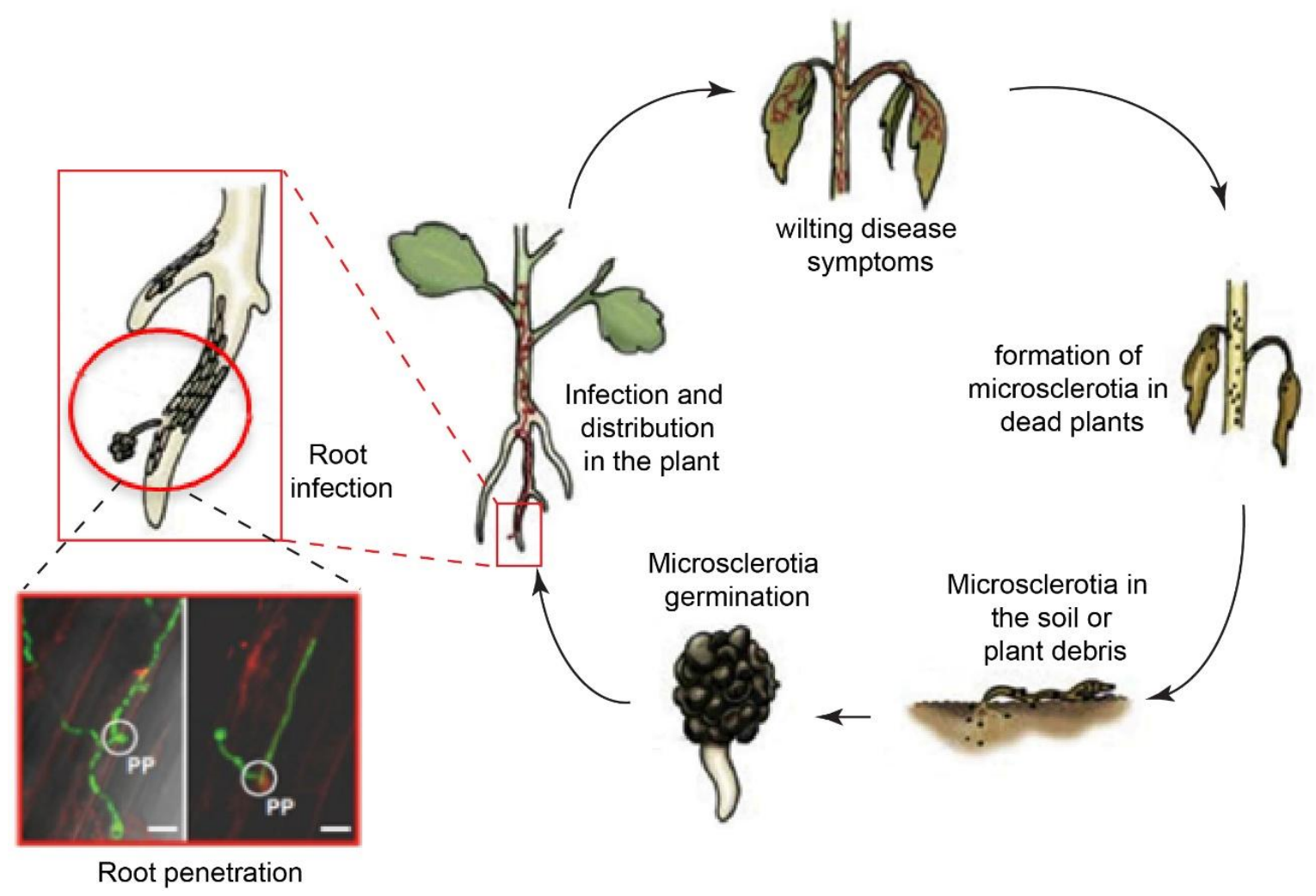

Figure 4. V. dahliae life cycle. The life cycle of $V$. dahliae starts when microsclerotia are stimulated by root exudates and germinate. Hyphae grow towards roots and penetrate root tips by forming penetration points (PP). Hyphae colonise the root cortex and enter the xylem vessels before forming asexual conidia and distributing in whole the plant. Symptoms like wilting, chlorosis and necrosis appear early in old leaves. Microsclerotia are formed in dead leaves, stems or in the soil until new plants are available. This figure is modified from Berlanger \& Powelson (2005) and Tran et al. (2014). 
The second step starts when the pathogenic fungi are already in the plant. Pathogenic fungal hyphae grow through cortical tissues toward vascular tissues and xylem vessels. The pathogen produces conidia which are transported from the root to the shoot with the xylem sap stream (Pegg \& Brady, 2002; Berlanger \& Powelson, 2005). During the transition, conidia germinate and develop inside the xylem vessels and enter the neighboring cells. Wherein, fungal hyphae produce more conidia to repeat this distribution process. Consequently, the pathogenic fungus occupies the cortical tissues, vascular cells, and the xylem vessels from roots to shoots. The disease symptoms such as wilting, chlorosis and necrosis start with appearing in lower and older parts of infected plants (Pegg \& Brady, 2002; Berlanger \& Powelson, 2005). Conidia have thin cell walls without melanin which cannot protect them against stress (Eynck et al., 2007). Therefore, microsclerotia are formed in the whole dying plant including roots, leaves, seeds, branches, and stems. If dead plants are incorporated into the soil, microsclerotia are gradually released during decomposition of the tissues and new disease cycles will start when new plants are cropped (Pegg \& Brady, 2002; Berlanger \& Powelson, 2005).

\subsection{Adhesion is essential for fungal pathogens}

Adhesion of fungi to host surfaces is an important pathogenicity factor for both, plant and animal pathogenic fungi (Hostetter, 2000). Adhesive proteins are glues known as the substratum adhesion of fungi. They promote fungi to stay on surfaces under effects of blowing or washing (Yan et al., 2011; Lin et al., 2015; Epstein \& Nicholson, 2016). Additionally, they increase the contact surface area between hosts and fungi which supports the penetration process of pathogens. Fungal mycelia require adhesion proteins for binding on root surfaces. Fungi may lose virulence if adhesion proteins are disrupted (Wang \& St Leger, 2007; Yan et al., 2011; Zhao et al., 2016). Mad1 and Mad2 were shown to be required for adhesion. Metarhizium anisopliae strains lacking MAD1 or MAD2 are not able to infect insects (Wang \& St Leger, 2007). Som1, a homolog of Flo8 in Magnaporthe oryzae, is essential for adhesion on rice leaves. The deletion strain of SOM1 is avirulent (Wang \& St Leger, 2007; Yan et al., 2011). Therefore, anti-adhesions can block adhesion protein functions like an antigen/antibody model without uptake into cells (Epstein \& Nicholson, 2016). Consequently, anti-adhesins might be used as a new strategy to control diseases. 


\subsubsection{Adhesion in yeasts}

Yeasts such as Saccharomyces cerevisiae or Candida albicans are unicellular fungi and exist in single vegetative cells, which can change to multi-cellular growth with the promotion of adherent proteins (Dranginis et al., 2007). Multi-cellular growth of yeasts can be found in flocculation, biofilm formation, and pseudohyphal processes (Braus et al., 2003; Verstrepen et al., 2003; Fichtner et al., 2007). These procedures require expression of specific cell wall associated adhesins which regulate cell-cell or cell-surface adhesion (Kobayashi et al., 1998; Braus et al., 2003; Verstrepen et al., 2003; Fichtner et al., 2007). Adhesive proteins are usually located on the surface of cell walls to facilitate the interaction of cell-cell or cell-surface adhesion (Rigden et al., 2004; Fichtner et al., 2007; Wang \& St Leger, 2007). Adhesive proteins in S. cerevisiae are well known. A family of flocculation genes (FLOs) was reported to play key roles in adherence (Kobayashi et al., 1996). There are two groups of flocculation genes. One group harbors a PA14 conserved domain including FLO1, FLO5, FLO9, and FLO10 (Kobayashi et al., 1996; Kobayashi et al., 1998). The PA14 domain sequence possesses a carbohydrate-binding function (Rigden et al., 2004). The FLO1, FLO5, and FLO9 genes share high sequence similarity (Dranginis et al., 2007). Flo1 is required for both, adhesion and flocculation (Kobayashi et al., 1998). Flocculation is useful in the brewing industry because flocculated cells are easily separated from the culture medium at the end of fermentation (Verstrepen et al., 2003). Therefore, one does not need a complicated filter system to take out cells from the culture medium.

The other group of flocculation genes contains FLO11 which is only responsible for initial surface adhesion providing only some cell layers (Fichtner et al., 2007). There are several transcription factors which bind to the promoter of FLO11 for activation or repression (Figure 5). Flo8 and Ste12 are found in the promoter region of FLO11 and required for expression of FLO11. Lack of either FLO8 or STE12 causes inactivation of FLO11. Sfl1 was reported to repress the expression of flocculation genes. It was found in the promoter region of FLO11. The Sfl1 binding site overlaps with that of Flo8 (Figure 5) (Octavio, 2009). 


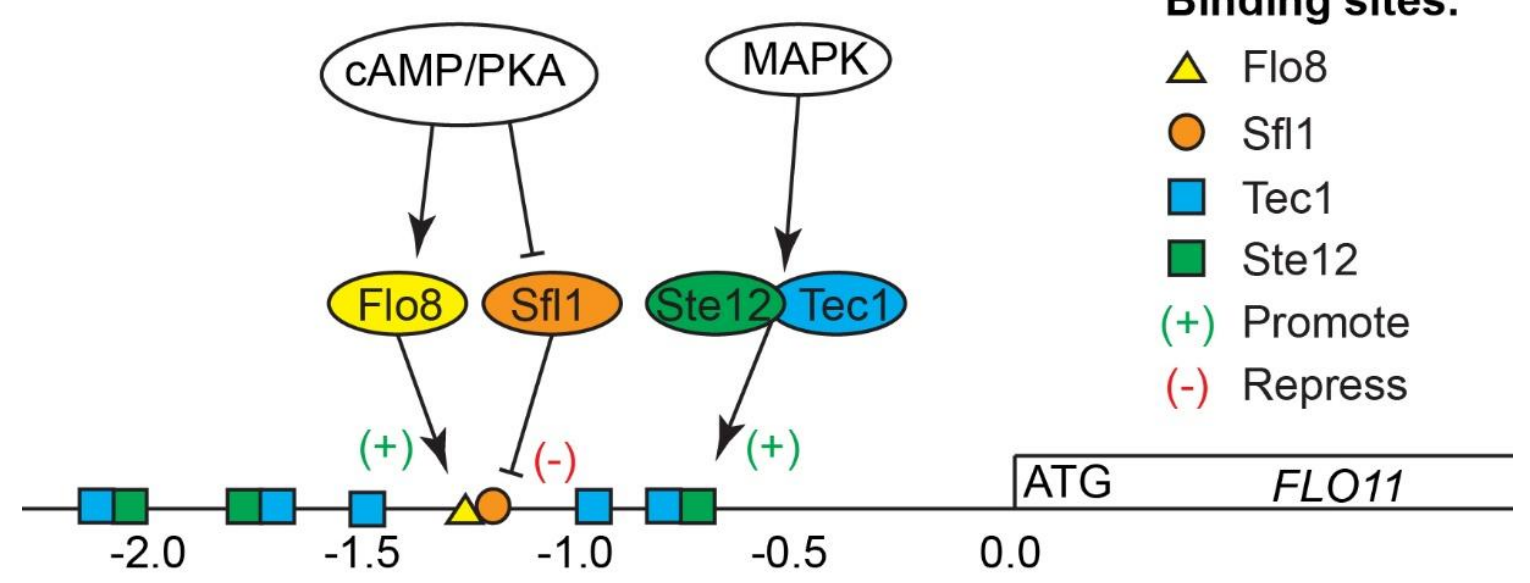

Binding sites:

$\triangle$ Flo8

Sfl1

Ste12

Promote

(-) Repress

Figure 5. Expression of FLO11 is controlled by cAMP/PKA and MAPK pathways. Mitogen activated protein kinase (MAPK) activates FLO11 via the Ste12 and Tec1 complex, whereas the cyclic AMP dependent protein kinase A cascade (cAMP/PKA) controls FLO11 through Flo8 and Sfl1. Flo8 activates expression of FLO11, whereas Sfl1 represses its expression. Flo8 activation and Sfl1 repressor binding sites are overlaping. This figure is modified from Octavio et al. (2009).

Flo8 is known as a transcription factor which is in downstream of the cyclic AMP dependent protein kinase A (cAMP/PKA) pathway, which was reported to control fungal development or pathogenicity in yeast $S$. cerevisiae or plant pathogenic fungus $M$. oryzae. The defect of FLO8 causes an inactivation of FLO1 and FLO11 genes in yeast. The point mutant of FLO8 gene shows a non-adhesive S. cerevisiae on agar plates and in liquid medium (Figure 6).

The primary colonisation of Candida albicans to host surfaces requires adherent proteins (Sundstrom, 2002). Several adhesive proteins are found such as enzymes, agglutinin-like sequence protein, integrins, and lectin-like protein (Cotter \& Kavanagh, 2000; Sundstrom, 2002; Bonfim-Mendonca et al., 2015). The outer cell wall layer is rich in glycosylated mannoproteins, which may contribute to host-fungus interactions. The protein mannosyltransferases (Pmt) can be found in the endoplasmic reticulum and mannosyltransferases (Mnt) in the Golgi (Timpel et al., 2000; Munro et al., 2005). Pmt is required for adherence to endothelial cells, whereas Mnt1 and Mnt2 are essential for human buccal epithelial cells. Both of them are involved in the glycosylation process (Timpel et al., 2000; Munro et al., 2005). A putative glycosidase (Csf4p) has a major role in adherence. Deletion of CSF4 reduces adhesion to mammalian cells (Alberti-Segui et al., 2004). CAMP65 encodes a putative $\beta$-glucanase, which is essential for adhesion to plastics (Sandini et al., 2007). Secreted aspartate proteinase such as Sap1, Sap2, Sap3, Sap4, and Sap6 may also contribute to adhesion (Naglik et al., 2004). 
(a)
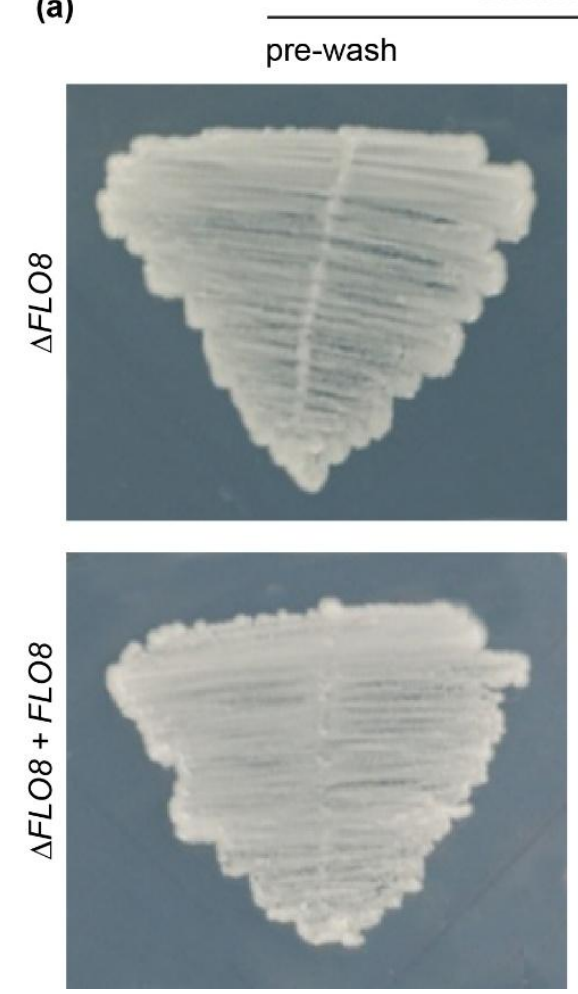

Biofilm formation

post-washed
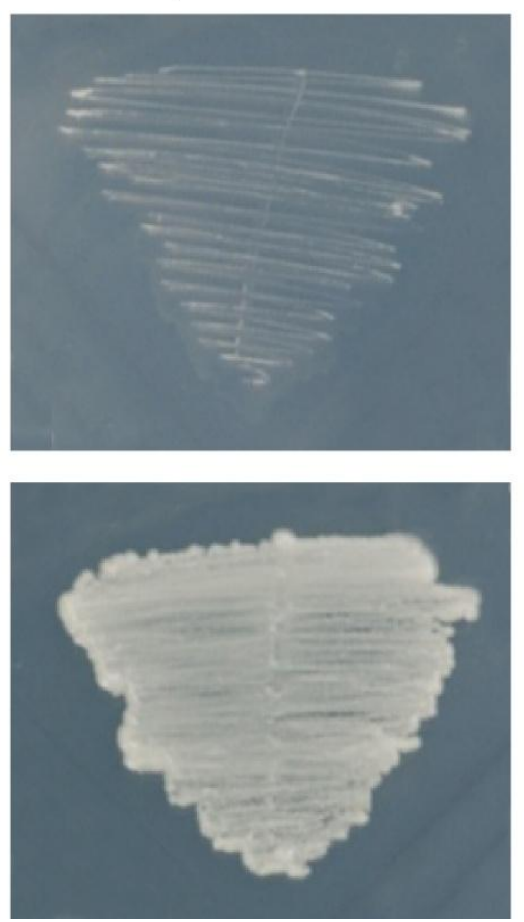

(b)

Flocculation
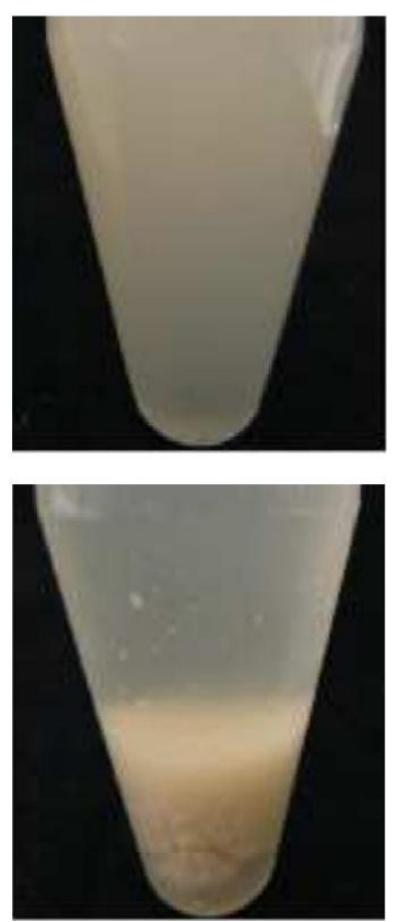

Figure 6. Adhesion of $\mathbf{S}$. cerevisiae on agar plates and in a liquid medium. (a) Biofilm formation on agar plates. The strain expressing FLO8 can produce biofilm on an agar plate, which is unable to be washed off, whereas the deletion strain of FLO8 is removed by washing. (b) Flocculation of a strain expressing FLO8 gene. This figure is modified from Tran et al. (2014) and from Lin et al. (2015).

The agglutinin-like sequence (Als) protein family was reported to be essential for adhesion to human epithelial cells (Fu et al., 2002; Sundstrom, 2002). The ALS gene family encodes a group of GPI-anchored proteins which are necessary for adhesion (Hoyer et al., 1998; Hoyer, 2001; Fu et al., 2002). There are nine ALS genes (ALS1-ALS9) in the $C$. albicans genome. Among Als proteins Als1p, Als3p, and Als5p are essential for adherence (Hoyer et al., 1998; Hoyer, 2001; Fu et al., 2002; Sundstrom, 2002). Als1p and Als3p are important for binding to endothelial and epithelial cells, whereas Als5p binds to extracellular matrix proteins (Hoyer et al., 1998; Fu et al., 2002; Epstein \& Nicholson, 2016). Structure and functions of Als1p are similar to Flo11 of S. cerevisiae (Fu et al., 2002; Sundstrom, 2002). Expression of $A L S 1$ is controlled by the transcription factor Efg1p, which is a key regulator of filamentation in C. albicans (Fu et al., 2002). Als1 and Hwp1 protein promote the biofilm formation process (Fan et al., 2013).

The hyphal wall protein 1 (Hwp1) is necessary for adhesion on the surface layer of the stratified squamous epithelium, which is found exclusively at the germ 
tube surface (Sharkey et al., 1999; Sundstrom, 2002). Hwp1 is required for biofilm formation in C. albicans. Deletion of HWP1 was defective in biofilm formation and effective human infection (Sharkey et al., 1999; Padovan et al., 2009). C. albicans binds to extracellular matrix proteins which might need the presence of integrin-like receptors (Int1) at the host surfaces (Calderone, 1998). Expression of the INT1 encoding integrin-like protein of $C$. albicans can reprogram non-adherent yeast S. cerevisiae to adhere to human epithelial cells (Calderone, 1998; Gale et al., 1998). The deletion of INT1 in C. albicans represses adhesion to human epithelial cells (Gale et al., 1998).

The epithelial adhesion protein family (EPA) contains seven proteins (Epa1Epa7) which harbor the PA14 conserved domain. Although there are seven members of the Epa family, only Epa1 is essential for binding to the epithelial cell during infection (Sundstrom, 2002; Dranginis et al., 2007; Zupancic et al., 2008). Epa1 is a $\mathrm{Ca}^{2+}$-dependent lectin which shows homology to Flo1 of $S$. cerevisiae and binds to $\mathrm{N}$-acetyllactosamine-containing glycoconjugates. Adhesion of the EPA1 deletion strain was reduced by 95\% compared to the wild-type strain (Sundstrom, 2002; Li \& Palecek, 2003). Other EPA genes are low expressed. For instance, EPA6 is not expressed in vitro, whereas its expression is enhanced during experimental urinary infection (Vitenshtein et al., 2016; Zajac et al., 2016).

\subsubsection{Adhesion and virulence in filamentous fungi}

Adhesion of filamentous fungi is not as well studied as adherence in yeast and is usually associated with virulence. Appressorium formation in filamentous fungi was reported to play important roles in adhesion and virulence (Figure 7) (Clergeot et al., 2001; Xue et al., 2002; Gourgues et al., 2004; Jeong et al., 2007; Yan et al., 2011). There are several transcription factors and cell wall proteins which were reported to have important roles in appressoria formation and promotion of adhesion and virulence (Clergeot et al., 2001; Xue et al., 2002; Gourgues et al., 2004; Jeong et al., 2007; Yan et al., 2011; Li et al., 2015a). The cAMP/PKA pathway and Pmk1 MAPK cascade are required for appressoria development and pathogenicity in the rice blast fungus (Lee \& Dean, 1993). The cAMP signaling pathway responds to an inductive signal from rice leaves such as hydrophobic surface and wax monomers, which is required for appressoria formation in M. oryzae (Zhou et al., 2012; Li et al., 2015a). Deletion of MAC1, a gene 
encoding an adenylate cyclase, cannot form appressoria, aerial hyphae, and conidia (Zhou et al., 2012; Li et al., 2015a). MoSOM1, a homolog of FLO8 in M. oryzae, is located downstream of the CAMP/PKA signaling pathway, which is regulated by MoMac1 (Yan et al., 2011). MoSom1 physically interacts with MoCdf1. Disruptions of MoSOM1 and MoCDF1 show defects in adhesion, appressoria formation and virulence (Yan et al., 2011). MoMPG1, a gene encoding a hydrophobin protein, is controlled by MoSom1, which is essential for fungal binding to plant leaves during plant infection (Beckerman \& Ebbole, 1996; Pham et al., 2016). Aspergillus fumigatus AfSomA, a homolog of Flo8, physically interacts with Ptab. Both of them play important roles in biofilm formation and adhesion (Lin et al., 2015). AfSomA is essential for virulence, whereas AfPtab is not (Lin et al., 2015). Adhesion of the fungi to the host surface and virulence require formation and function of appressoria (Braun \& Howard, 1994; Beckerman \& Ebbole, 1996; Xue et al., 2002; Li et al., 2015a).

Genes specifically expressed in appressoria of $M$. grisea were examined. There are 72 genes only expressed in mature appressoria including GAS1 and GAS2 (Lu et al., 2005). Gas1 and Gas2 are not essential for appressoria formation, but they are required for appressorium penetration and lesion development (Xue et al., 2002; Lu et al., 2005). A main polysaccharide component of the cell wall, which was reported to play important roles in appressoria formation is chitosan. Chitosan can be formed from chitin under the catalysis of chitin deacetylases (CDAs) (Pochanavanich \& Suntornsuk, 2002). Chitosan is reported to localise in the germ tube and appressoria. Deletion strains of CDA genes showed loss of chitin deacetylation, reduced adherence and appressoria formation on an artificial hydrophobic surface (Geoghegan \& Gurr, 2016). 


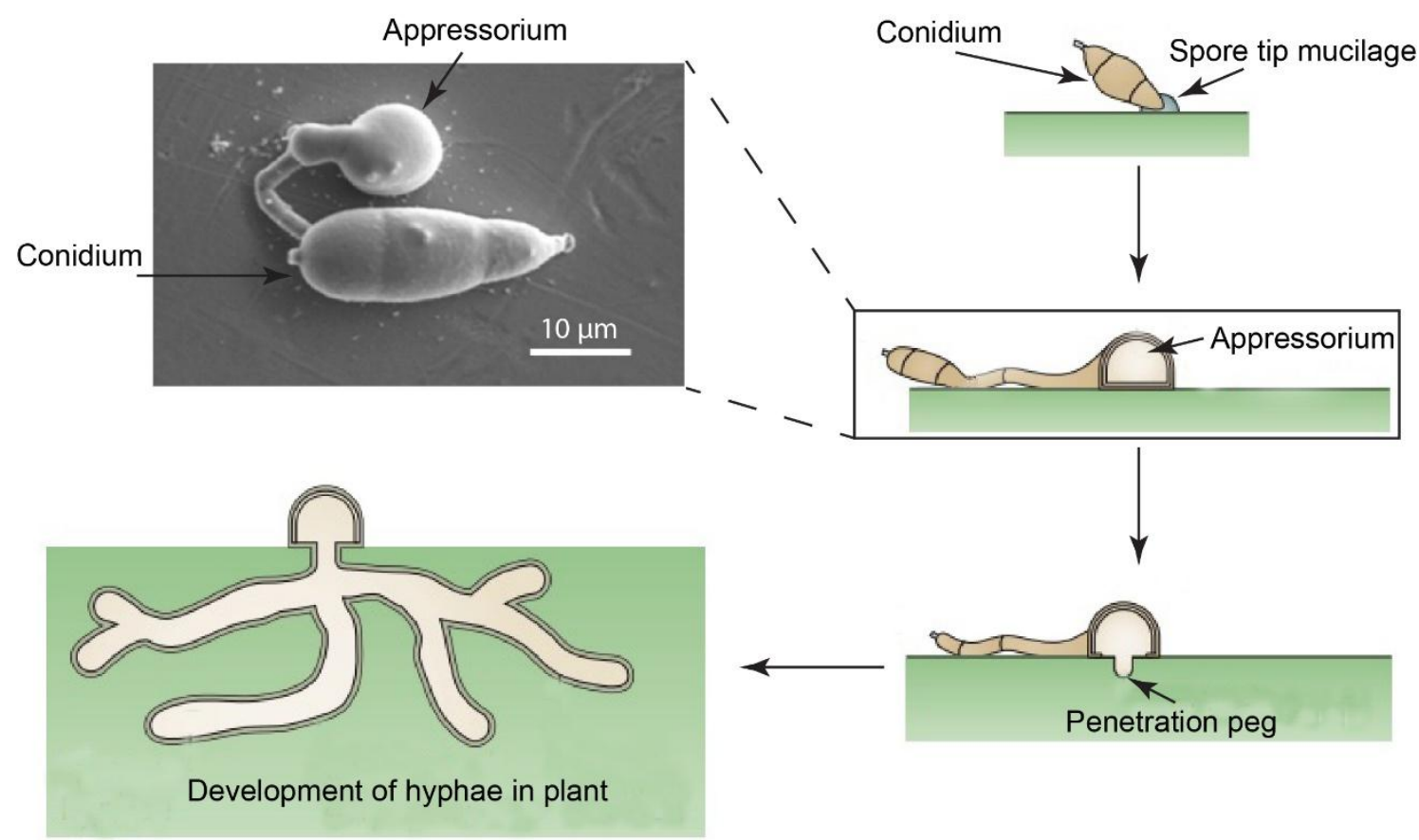

Figure 7. Appressoria are required for the rice blast fungus Magnaporthe oryzae infection. Spores attach to the hydrophobic cuticle by spore tip mucilage, germinate and produce a narrow germ tube. Appressoria are formed in the tip of the germ tube. Appressoria become melanised and substantial turgor before forming a narrow penetration peg at the base. The penetration peg punctures the cuticle and enters the rice epidermis. Hyphae continue developing, moving from cell to cell, and inducing disease symptoms in plant leaves. This figure is modified from Skamnioti \& Gurr (2007) and from Wilson \& Talbot (2009).

The tetraspanin protein family was reported to join membrane signalling complexes controlling cell differentiation, motility, and adhesion (Clergeot et al., 2001). The PLS1 gene encodes a putative integral membrane protein and is related to the tetraspanin family. It is localised in plasma membranes and vacuoles. Pls1 plays an appressorial function. This protein is essential for penetration of $M$. grisea into host leaves (Clergeot et al., 2001). BcPls1, a homolog of Pls1 in Botrytis cinerea, was also reported to be involved in the penetration process into host plant leaves (Gourgues et al., 2004). Hydrophobin proteins were shown to play crucial roles in cell morphogenesis and pathogenicity in several plant pathogenic fungi including M. oryzae (Linder et al., 2005; Pham et al., 2016). Mpg1 and Mhp1 are hydrophobin proteins, which are highly expressed during rice blast infection. They promote spore adhesion and penetration into the host plant (Beckerman \& Ebbole, 1996; Pham et al., 2016). The fasciclin family protein MoFlp1 contains a glycosylphosphatidylinositol (GPI) anchor and is localised on the vacuole membrane. The deletion strain of MoFLP1 showed defects in adhesion, 
appressorium turgor, and virulence (Liu et al., 2009). Adhesion protein and appressoria formation are also studied in other pathogenic fungi (Hwang et al., 1995; Wang \& St Leger, 2007; Zhang et al., 2009).

In Colletotrichum gloeosporioides, CAP20 is known to be expressed during appressoria formation. The CAP20 deletion strain does not show defects in appressoria formation but its function might be unfulfilled. The deletion strain showed a drastic decrease in virulence on avocado and tomato fruits (Hwang et al., 1995). Metarhizium anisopliae adherence proteins Mad1 and Mad2 are produced on the conidial surface. Expression of MAD1 and MAD2 caused yeast to adhere to insect cuticle or a plant surface. Mad1 and Mad2 are required for adhesion in $M$. anisoplia (Voegele et al., 2005). The disruption of MAD1 caused delayed germination, suppressed blastospore formation, and greatly reduced virulence to the caterpillar. The deletion of Mad2 blocked adhesion of $M$. anisopia to plant epidermis (Wang \& St Leger, 2007). BbHog1 encodes a functional homolog of yeast high-osmolarity glycerol (HOG), which regulates expression of hydrophobin genes such as HYD1 and HYD2 in Beauveria bassiana (Zhang et al., 2009).

\subsubsection{Adhesion and virulence in $V$. dahliae}

The plant pathogen $V$. dahliae enters the plant roots without forming appressoria. Its hyphae can directly go into or form hyphopodia before entering the roots (Figure 8 ). There are several genes which have been reported to be related to adhesion and virulence in $V$. dahliae. The VdPLS1 gene encoding a tetraspanin protein was specifically expressed in hyphopodia, which was shown to function as an adapter protein for the recruitment and activation of VdNoxB. Both, VdPIs1 and VdNoxB, were required for hyphopodia peg formation (Figure 8). Deletion strains of VdPLS1 and VdNOXB were unable to colonise cotton plant roots and to cause disease symptoms. 
(a)

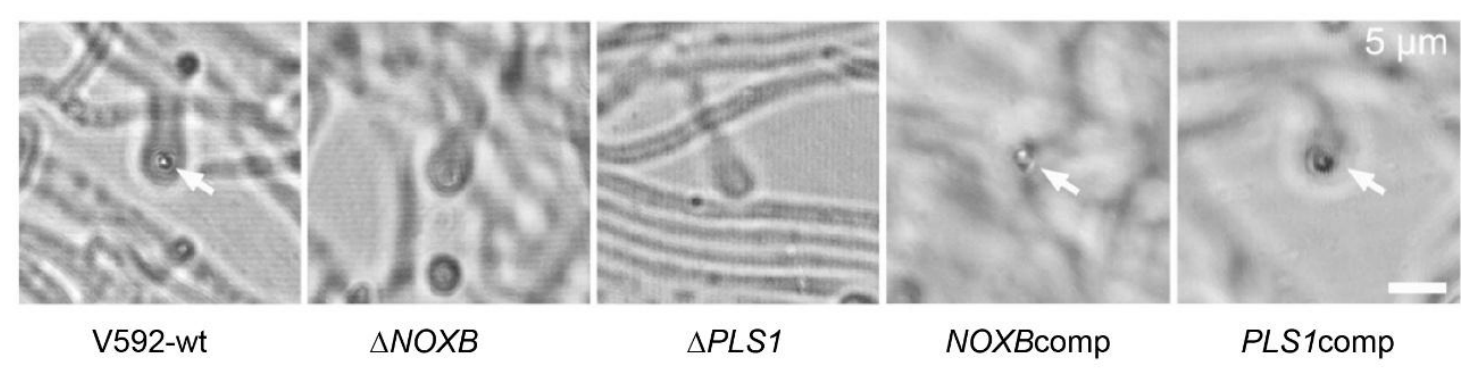

(b)

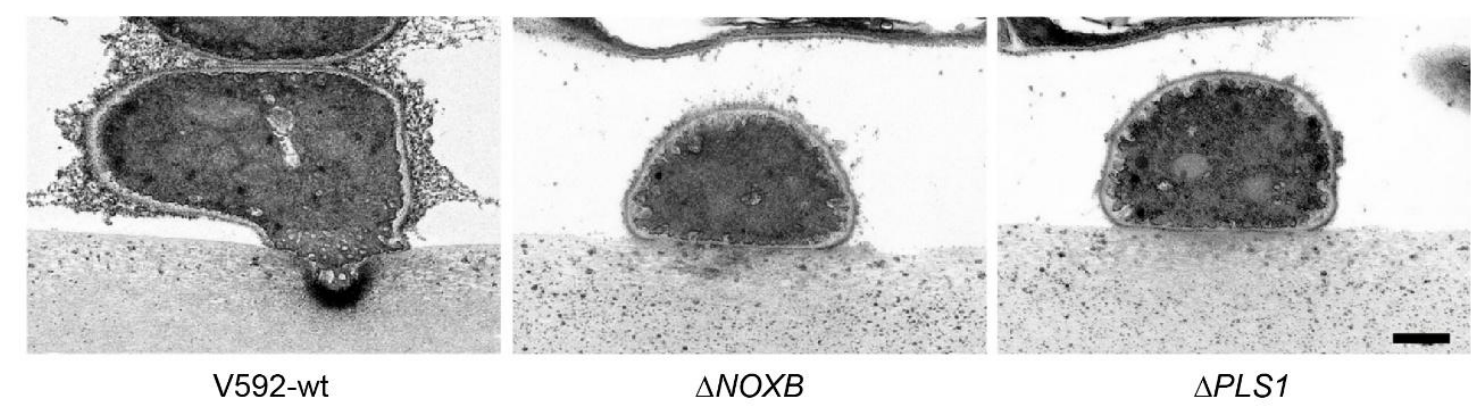

Figure 8. NoxB and Pls1 are essential for hyphopodia peg formation. (a) Hyphopodia peg development on cellophane membrane at 2 dpi. Hyphopodia pegs indicated by the arrowhead were only observed in V592-wt and complementation strains. They are not found in neither of the deletion strains of NOXB nor PLS1. (b) Cerium deposits were observed at the tip of hyphopodia peg in V592-wt, whereas they were not detected in both deletion strains of NOXB and PLS1. Scales bars are $5 \mu \mathrm{m}$. This figure is modified from Zhao et al. (2016).

Transcription factor Mcm1 belongs to the SRF subfamily of MADs-box family (Xiong et al., 2016). Mcm1 is known as a key regulator of conidiation, microsclerotia formation, and virulence. Conidia production in the MCM1 deletion strain was reduced by $80 \%$ compared with the wild-type strain. No microsclerotium was detected in the deletion strain of MCM1, whereas they were detectable in the wild-type strain. Deletion of VdMCM1 showed defects in adhesion of $V$. dahliae to abiotic surfaces and virulence on tomatoes. This deletion strain also failed to adhere to plant roots and to infect smoke trees. It promotes, however, hyphal growth and cell wall integrity (Xiong et al., 2016). Putative adhesion genes from Verticillium were also screened using defective FLO8 S. cerevisiae (Tran et al., 2014).

S. cerevisiae with a defect $F L O 8$ gene was used as a tool to screen for adhesion genes from Verticillium longisporum. In total twenty-two genes from $V$. longisporum were found to be able to reprogram the non-adhesive yeast to adherence (Figure 9a). However, only nineteen genes had predicted cellular functions and homologs to these genes could also been identified in $V$. dahliae. The predicted cellular 
functions differ and include transcription regulation, signaling, protein trafficking, morphogenesis, pigmentation, and unknown functions (Figure 9b). None of them revealed any typical adhesion characteristics like GPI anchor or signal peptides (Tran et al., 2014). Six out of the nine are putative transcription regulatory genes, which were named $\underline{V} e r t i c i l l i u m$ transcription activator of adhesion (VTA1 - VTA6). Vta2 encodes a $\mathrm{C}_{2} \mathrm{H}_{2}$ zinc finger protein, which is the homolog of the $M$. grisea Con-7 regulator. This gene is required for colonisation on plant roots and virulence of $V$. dahliae (Tran et al., 2014). The deletion of VTA2 resulted in a strain which was unable to colonise roots and cause disease symptoms. Additionally, it was defective in conidia formation in SXM (Tran et al., 2014). Vta3 is a putative suppressor of A-kinase (Sak1), encoding a Winged helix-turn-helix DNA binding protein.

(a)
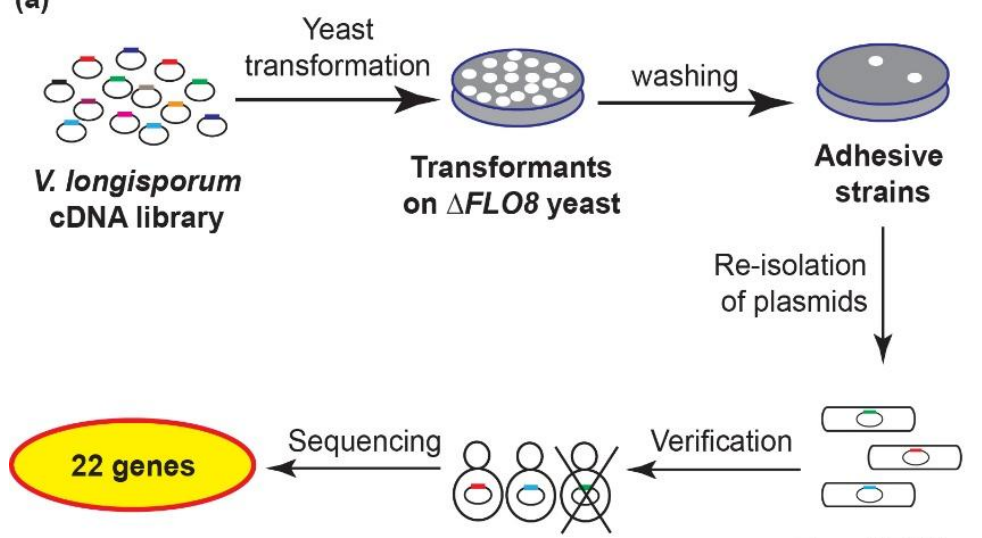

Adhesive clones (b) Functional groups

\begin{tabular}{|l|c|}
\hline Predicted functions & Number \\
\hline $\begin{array}{l}\text { Morphogenesis and } \\
\text { pathogenicity }\end{array}$ & 2 \\
Pigmentation & 1 \\
$\begin{array}{l}\text { Regulation of transcription } \\
\text { and development }\end{array}$ & 9 \\
$\begin{array}{l}\text { Signaling and protein } \\
\text { trafficking }\end{array}$ & 3 \\
Nucleic acid binding & 2 \\
Unknown functions & $\mathbf{5}$ \\
\hline
\end{tabular}

E. coli DH5a

Figure 9. A FLO8 defective $S$. cerevisiae strain was used as a tool to screen for adhesion genes in Verticillium. (a) V. longisporum cDNA library under the control of the yeast specific galactokinase GAL1 promoter was transferred into the non-adherent yeast deletion strain of FLO8. The transformants were grown on agar plates containing galactose and were gently washed with water. Plasmids from adhesive transformants were re-isolated, transformed into $E$. coli, and followed by verifying adhesion of yeast. The confirmed genes were sequenced and analysed. Twenty-two genes were found during the screen. (b) Predicted gene functions with Pfam/interProScan or BLAST search. Nine genes were putative transcription factors. This figure is modified from Tran et al. (2014). 


\subsubsection{Wing helix-turn-helix DNA binding proteins}

Wing helix-turn-helix DNA binding proteins harbor a related winged helixturn-helix DNA binding motif which consists of two wings (W1, W2), three alpha helices $(\mathrm{H} 1, \mathrm{H} 2, \mathrm{H} 3)$ and three beta-sheets $(\mathrm{S} 1, \mathrm{~S} 2, \mathrm{~S} 3)$ arranged in the order $\mathrm{H} 1$ S1-H2-H3-W1-S3-W2 (Figure 10) (Gajiwala \& Burley, 2000). The wing W1 and helix $\mathrm{H} 3$ response DNA binding. Wing W1 of Rfx1 bind to major groove whereas helix $\mathrm{H} 3$ attach to minor groove of DNA. The wing helix-turn-helix DNA binding motif is highly conserved in fungi.

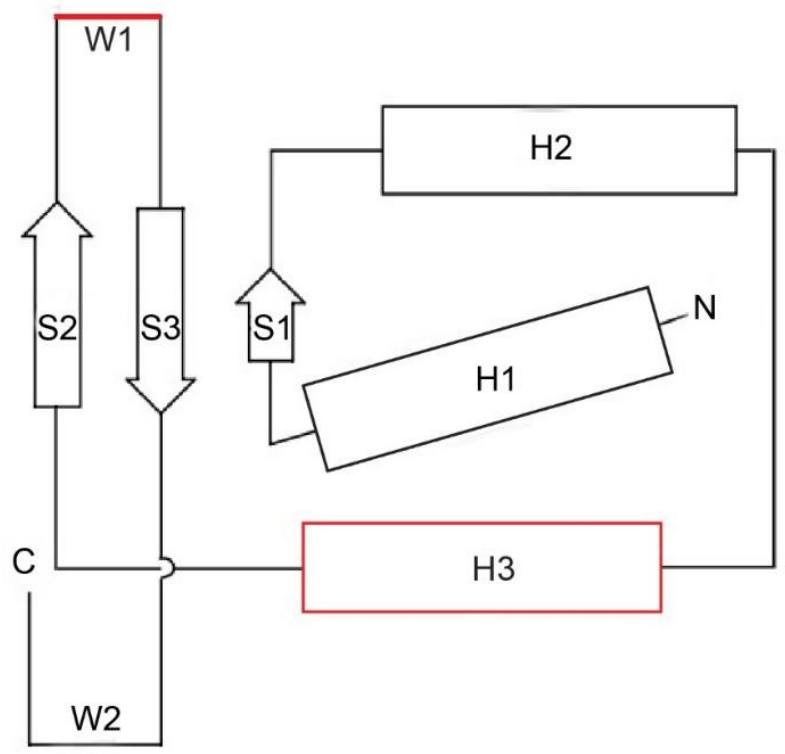

Figure 10. Topology of the winged helix fold. The winged helix motif contains two wings (W1 and $\mathrm{W} 2)$, three $\alpha$-helices $(\mathrm{H} 1, \mathrm{H} 2$, and $\mathrm{H} 3)$, and three $\beta$-strands (S1, S2, and $\mathrm{S} 3$ ), which are arranged in order H1-S1-H2-H3-S2-W1-S3-W2. The wing W1 and helix H3 of $\mathrm{Rfx} 1$ are essential for DNA binding. The wing $\mathrm{W} 1$ binds to major groove whereas helix $\mathrm{H} 3$ overlies the minor groove of the X-box of DNA. The N-terminus is largely helical, whereas the C-terminus is composed of two strands and two large loops of wings. Red colour indicates the binding site of this motif to the X-box of DNA. This figure is modified from Gajiwala \& Burley (2000).

The winged helix-turn-helix DNA binding domain is well studied in yeasts (Min et al., 2014). In S. cerevisiae, the ScRfx1 transcription factor is reported to bind to the promoters of target genes and is involved in DNA repairing (Emery et al., 1996). ScRfx1p recruits the repressors Tup1p and Cyc8p to inhibit the transcription of target genes (Emery et al., 1996). This complex directly interacts with Sfl1, a suppressor gene for flocculation, which plays a major role in suppressing the expression of the flocculation gene FLO11 (Emery et al., 1996). The winged helix- 
turn-helix DNA binding domain is also studied in filamentous fungi. In Penicillium chrysogenum, PcRfx1 binds to the promoter region of penicillin biosynthetic genes. Lack of PCRFX1 caused a reduction of penicillin production (Bugeja et al., 2010). In Fusarium graminearum, FgRfx1 is required for aerial hyphal formation, conidia formation, and virulence. In V. dahliae, the function of Vta3, a homolog of ScRfx1, has not been studied yet, whereas its putative interaction partner VdCyc8 was reported to play important roles in conidia and microsclerotia formation, as well as in virulence. Vta3 can rescue defective FLO8 in S. cerevisiae (Tran et al., 2014) and thereby might play important roles in $V$. dahliae adhesion.

\subsection{Regulation of conidia and microsclerotia formation}

Gene expression during microsclerotia formation was examined (Neumann \& Dobinson, 2003; Duressa et al., 2013), however, the mechanism of that process in Verticillium has not been described yet. Genes especially required for conidiation are well studied in N. crassa, A. nidulans, and A. fumigatus (Roberts \& Yanofsky, 1989; Tao \& Yu, 2011; Son et al., 2013), whereas they have not been explained in Verticillium yet. Some factors of conidia and microsclerotia formation were described in V. dahliae (Klimes \& Dobinson, 2006; Tran et al., 2014; Zhao et al., 2016).

\subsubsection{Regulation of conidiation}

Conidia play a role in distribution of the pathogen in planta or in the environment through water or the air (Pegg \& Brady, 2002; Berlanger \& Powelson, 2005). In Neurospora crassa, conidia formation requires the expression of CON family genes CON-1 to CON-13. Con-6, Con-8, Con-10, Con-11, and Con-13 play a unique role during early conidia formation. Con- 6 and Con-8 are expressed early during early conidia formation (Roberts \& Yanofsky, 1989). Deletion of MgCON-6 from M. grisea blocked conidia formation (Shi \& Leung, 1995). MgCon-7 from M. grisea and Vta2, a homolog of Con-7 in V. dahliae, were shown to play an important role in conidia formation (Shi \& Leung, 1995; Tran et al., 2014).

Transcriptional factors $\mathrm{Brl} A, \mathrm{AbaA}$, and WetA are reported to play a central role in asexual development in Aspergillus. BrlA is essential for vesicle formation at the tip of aerial hyphae and controls the expression of ABAA and WETA (Tao and $Y u, 2011) . A b a A$ is needed for phialide differentiation from vegetative hyphae and it 
activates WETA which is required for conidiophore maturation (Figure 11). The deletion strains of $A b a A$ can produce metulae, but they were unable to generate phialides (Tao \& Yu, 2011; Son et al., 2013). AbaA and WetA promote VOSA which is required for trehalose accumulations and conidia maturation. VosA was reported to repress the expression of $B R L A$ (Tao and $Y u, 2011)$. In Verticillium, the homologs of $\mathrm{BrlA}, \mathrm{AbaA}$, and WetA are found. But their function in conidia formation has not been studied yet, but some proteins are known to be related to conidia formation such as Pls1, Vta2, Mcm1, Vdh1, and Vmk1 (Rauyaree et al., 2005; Klimes \& Dobinson, 2006; Tran et al., 2014; Xiong et al., 2016; Zhao et al., 2016).

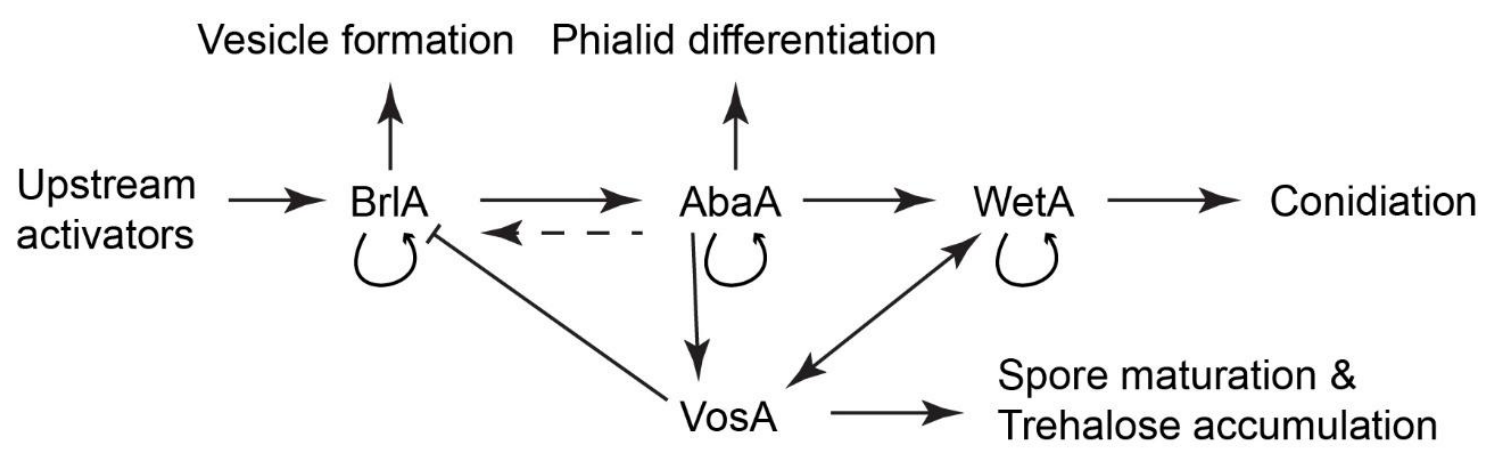

Figure 11. The central developmental pathway of conidia formation in $\boldsymbol{A}$. nidulans. $\mathrm{BrlA}$ is essential for vesicle formation and regulates $A B A A$. AbaA is required for phialide differentiation and control WETA which is necessary for conidia formation. VOSA is regulated by $A b a A$ and WetA and represses $B R L A$ expression during vegetative growth. Arrow heads indicate activating effects. Dashed line shows proposed interaction. This figure is modified from Tao \& Yu (2011).

\subsubsection{Regulation of microsclerotia formation}

Microsclerotia have thick cell walls with dense melanin layers, which can survive up to 15 years in the soil without the presence of the hosts (Pegg \& Brady, 2002; Berlanger \& Powelson, 2005; Fradin et al., 2009). In previous studies, genes specifically expressed during microsclerotia formation were examined (Neumann \& Dobinson, 2003; Duressa et al., 2013). 153 genes were only expressed in induction medium for microsclerotia production. Approximately $50 \%$ of them were hypothetical proteins with unknown function (Duressa et al., 2013). The others are putatively involved in pigmentation and transcription factors associated with pigment production, secondary metabolic enzymes, cell growth, morphogenesis, signaling, carbohydrate active enzymes and transport proteins (Neumann \& Dobinson, 2003; Duressa et al., 2013). Additionally, there are several genes which 
were reported to control the formation of microsclerotia such as the Cyc8 glucose repression mediator protein (VdCyc8) (Li et al., 2015b), the mitogen-activated protein kinase protein (VdVmk1) (Rauyaree et al., 2005), the mitogen-activated protein kinase (VdPbs2) (Tian et al., 2016), the transfer membrane mucin (VdMsb) (Tian et al., 2014), the homolog of a high osmolarity glycerol (VdHog1) (Wang et al., 2016), the calcineurin-responsive zinc finger transcription factor (VdCrz1) (Xiong et al., 2015), the MADs-box transcription factor (VdMcm1) (Xiong et al., 2016), and the hydrophobin protein (Vdh1) (Klimes \& Dobinson, 2006). Especially, the hydrophobin protein Vdh1 is known as a key factor for microsclerotia formation. It is expressed during microsclerotia formation. Deletion of $\mathrm{VDH} 1$ does not affect growth or virulence, but the deletion strain is unable to form microsclerotia (Klimes \& Dobinson, 2006).

\subsection{Aim of this work}

The infection of the host by pathogenic fungi requires penetration and colonisation processes (Tran et al., 2014; Zhao et al., 2016). Defects in the penetration or colonisation step blocks plant infection by pathogenic fungi (Yan et al., 2011; Tran et al., 2014; Zhao et al., 2016). To penetrate the root surface, adhesive proteins are needed at several stages during the host-parasite interaction (Braun \& Howard, 1994; Hostetter, 2000). However, the understanding of the adhesion process of Verticillium is still limited. Adhesion is well studied in S. cerevisiae. The flocculation genes (FLOs) encode cell wall-associated adhesions. FLO1 and FLO11 promote flocculation or substrate adhesion, respectively (Liu et al., 1996; Verstrepen \& Klis, 2006; Fichtner et al., 2007; Van Mulders et al., 2009). Flo1 is required for both, adhesion and flocculation, whereas Flo11 is only responsible for initial surface adhesion providing only some cell layers (Fichtner et al., 2007). Both of them are activated by Flo8 which is located downstream of the cAMP/PKA pathway (Rupp et al., 1999; Fichtner et al., 2007).

The FLO8 deletion strain BY4742 (Euroscarf, Frankfurt, Germany) does not produce a functional Flo8 protein and is therefore unable to adhere to agar plates or between cells in liquid medium (Liu et al., 1996; Kim et al., 2014). This strain was used to dissect adhesion in Verticillium and to isolate specific genes required for adherence which might also control early plant infection. Twenty-two genes were reported by Tran et al. to be able to reprogram non-adhesive yeasts to adhere to 
agar plates. Nine teen out of the twenty-two genes were also identified in V. dahliae (Tran et al., 2014). Six of them are supposed to be transcription activator genes, which were named as Verticillium transcription activator of adhesion (VTA) (Tran et al., 2014). The homolog of Vta3 in Fusarium graminearum is reported to play key roles in DNA damage repair, conidiation, fungal growth, and pathogenicity (Huang et al., 1998; Min et al., 2014), whereas the function in adhesion has not been studied yet. Low expression of SOM1 and expression of VTA3 can rescue adhesion in nonadhesive yeast strains.

The first aim of this study was to analyse the function of Som1 and Vta3 in adhesion of $S$. cerevisiae by testing the expression of related adhesion genes. The function of Som1 and Vta3 in adhesion and virulence in $V$. dahliae was the second part of this work. Genetical, cell biological, proteomic assay and plant pathogenicity approaches were applied. Gene deletion and complementation strains were generated. Som 1 and Vta3 functions were investigated by comparing the deletion strain to the wild-type and complementation strains. The wild type and deletion strain were labelled with GFP to test root infection. Root infection was examined with scanning electron microscopy. Proteins, which are down regulated in the deletion of SOM1 were compared by a proteomic assay between cell lysates of the wild-type and deletion strains. The expression of relevant genes for adhesion, conidiation, microsclerotia formation, oxidative stress response and virulence was tested by real-time PCR. Interaction partners of Som 1 and Vta3 were identified by a pull-down assay using the GFP-trap. The AfSom1 in A. fumigatus is required for adhesion and virulence (Lin et al., 2015). The third part of this study was to test whether AfSomA and Som1 share similar functions in plant and human pathogenic fungi. The functional complementation study by expressing AfSOMA in the SOM1 deletion strain of $V$. dahliae was performed to examine the change of adhesion, growth, and the formation of aerial hyphae, conidia, and microsclerotia. 


\section{Materials and Methods}

\subsection{Materials}

\subsubsection{Chemicals}

Chemicals used in this study were purchased from Sigma-Aldrich (Deisenheim, Germany), Merck (Darmstadt, Germany), Roche Diagnostics GmbH (Mannheim, Germany), Invitrogen GmbH (Karlsruhe, Germany), AppliChem GmbH (Darmstadt, Germany), and Serva Electrophoresis GmbH (Heidelberg, Germany).

Enzymes used in this study were supported by Thermo Fisher Scientific GmbH (St. Leon-Rot/Schwerte, Germany) and Biolab inc. (Massachusetts, USA).

\subsubsection{Primers}

Primers used in this study were synthesized by Eurofins MWG GmbH (Ebersberg, Germany) and are listed in Table 1.

\section{Table 1. Primers used in this study}

q: RT-PCR primers, F: forward primer, R: reverse primer, restriction sites and overhang were underlined.

\begin{tabular}{|c|c|c|}
\hline Primer & 5'-3' direction & Reference \\
\hline \multicolumn{3}{|l|}{ For knockout } \\
\hline gdpA-NAT-F (Sacl) & GGG GAG CTC ACT AGT GGT ACC AGC GC & \multirow{2}{*}{ This study } \\
\hline gdpA-NAT-R (Apal) & GGG GGG CCC GGA TCC TCA GGG GCA GGG & \\
\hline SOM1-P1 (Pacl) & GGG TTA ATT AAC GGA GGA TGA TTG AGT AGC C & \multirow{2}{*}{ This study } \\
\hline SOM1-P2 (Spel) & GGG ACT AGT GAG ACA AGG TGG GAG TGG AA & \\
\hline SOM1-P3 (Xbal) & GGG TCT AGA CAA TGT GCC ATG ACG CTA TC & \multirow{2}{*}{ This study } \\
\hline SOM1-P4 (Sbfl) & 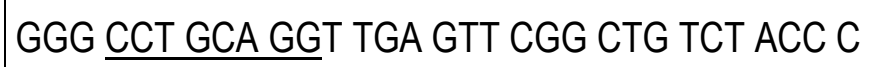 & \\
\hline VTA3-P1 (Pacl) & GGG TTA ATT AAT CAG CTG AAA TGT GGA ATC G & \multirow{2}{*}{ This study } \\
\hline VTA3-P2 (Spel) & GGG ACT AGT CCG GCT TGT GAT GAA GCT AT & \\
\hline VTA3-P3 (Xbal) & GGG TCT AGA CTT TTC GCT TTA GGG GGT TC & \multirow{2}{*}{ This study } \\
\hline VTA3-P4 (Sbfl) & GGG CCT GCA GGG CGA GTT TTC GGT AGG TGA G & \\
\hline
\end{tabular}


Table 1. (Continued)

q: RT-PCR primers, F: forward primer, R: reverse primer, restriction sites and overhang were underlined.

\begin{tabular}{|c|c|c|}
\hline Primer & 5'-3' direction & Reference \\
\hline \multicolumn{3}{|c|}{ For complementation } \\
\hline $\begin{array}{l}\text { SOM1comp-F } \\
\text { (Xbal) }\end{array}$ & GGG TCT AGA GGG GCA TTG TGT GTA CCT CT & \multirow{2}{*}{ This study } \\
\hline $\begin{array}{l}\text { SOM1comp-R } \\
\text { (Sbfl) }\end{array}$ & 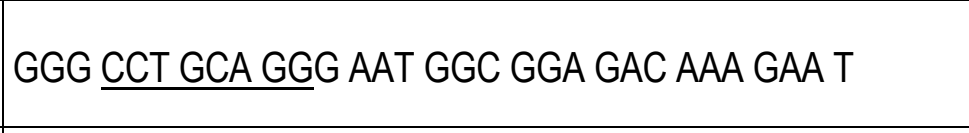 & \\
\hline $\begin{array}{l}\text { SOM1-Y-F } \\
\text { (Xhol) }\end{array}$ & 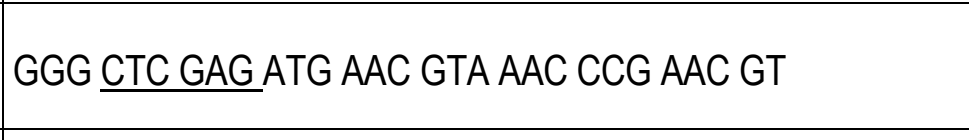 & \multirow[t]{2}{*}{ This study } \\
\hline $\begin{array}{l}\text { SOM1-Y-R } \\
\text { (HindIII) }\end{array}$ & 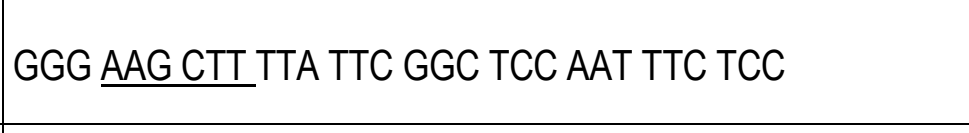 & \\
\hline ScFLO8-F (Xbal) & 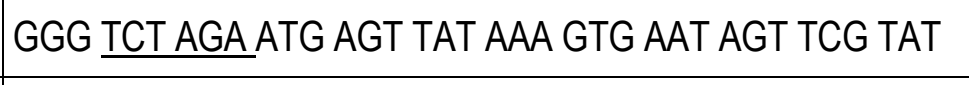 & \multirow[t]{2}{*}{ This study } \\
\hline ScFLO8-R (Sbfl) & 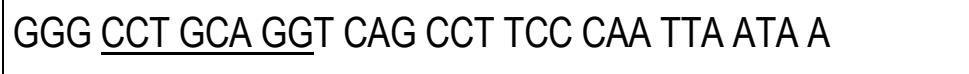 & \\
\hline $\begin{array}{l}\text { VTA3comp-F } \\
\text { (Xbal) }\end{array}$ & GGG TCT AGA GCC ATG AAG TGA CCG ACT TT & \multirow[t]{2}{*}{ This study } \\
\hline $\begin{array}{l}\text { VTA3comp-R } \\
\text { (Sbfl) }\end{array}$ & 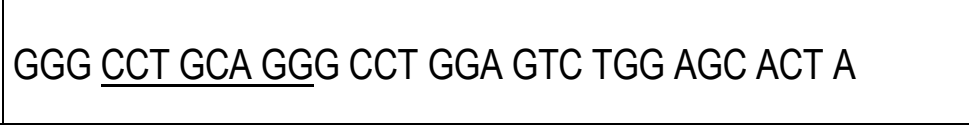 & \\
\hline \multicolumn{3}{|l|}{ For localisation } \\
\hline SOM1GFP-F & ATC CTT CTT TCT AGC AGG GGC ATT GTG TGT ACC TCT & \multirow{2}{*}{ This study } \\
\hline SOM1GFP-R & $\begin{array}{l}\text { GCC CTT GCT CAC CAT ACC ACC GCT ACC ACC TTC GGC } \\
\text { TCC AAT TTC TCC }\end{array}$ & \\
\hline FLO8GFP-F & $\begin{array}{l}\text { TGA CAT CAC CCT CGA GAT GAG TTA TAA AGT GAA TAG } \\
\text { TTC GTA TCC AG }\end{array}$ & \multirow{2}{*}{ This study } \\
\hline FLO8GFP-R & $\begin{array}{l}\text { GCC CTT GCT CAC CAT ACC ACC GCT ACC ACC GCC TTC } \\
\text { CCA ATT AAT AAA ATT GAA A }\end{array}$ & \\
\hline SOMAGFP-F & $\begin{array}{l}\text { TGA CAT CAC CCT CGA GAT GAA TCA GAT GAA TGT GAC } \\
\text { GGG }\end{array}$ & \multirow[t]{2}{*}{ This study } \\
\hline SOMAGFP-R & $\begin{array}{l}\text { GCC CTT GCT CAC CAT ACC ACC GCT ACC ACC TAA GCC } \\
\text { ATC TCC GGC GC }\end{array}$ & \\
\hline VTA3GFP-F & ATC CTT CTT TCT AGC AGC CAT GAA GTG ACC GAC TTT & \multirow{2}{*}{ This study } \\
\hline VTA3GFP-R & $\begin{array}{l}\text { GCC CTT GCT CAC CAT ACC ACC GCT ACC ACC TTG CTC } \\
\text { GAT GCC CTG CC }\end{array}$ & \\
\hline
\end{tabular}


Table 1. (Continued)

q: RT-PCR primers, F: forward primer, $R$ : reverse primer, restriction sites and overhang were underlined.

\begin{tabular}{|c|c|c|}
\hline Primer & 5'-3' direction & Reference \\
\hline \multicolumn{3}{|c|}{ For sequencing } \\
\hline SOM1seq 1 & CCC AAA ACC TTC TCA AGC AA & \multirow{2}{*}{ This study } \\
\hline SOM1seq 2 & CTG ATT CTG CTT TGC CTG GT & \\
\hline SOM1seq 3 & CAT GGC CAA CAA TCA GAA CA & \multirow{2}{*}{ This study } \\
\hline SOM1seq 4 & AAA GTT TGG TGC CAT AGA AGG A & \\
\hline SOM1seq 5 & CAA TCT CGC GGC TCT CCT A & \multirow{2}{*}{ This study } \\
\hline SOM1seq 6 & CCC GGA TTC TAG AAC TAG TGG A & \\
\hline VTA3seq 1 & CCC AAA CCT GTT CTG GAC AC & \multirow{2}{*}{ This study } \\
\hline VTA3seq 2 & GAG CGA CAG CTC AAT GAC AA & \\
\hline VTA3seq 3 & TTC TCA ACC CAG CGA GTT TC & \multirow{2}{*}{ This study } \\
\hline VTA3seq 4 & GGT CTT CAG TCC TGG GAA CA & \\
\hline VTA3seq 5 & GTC ATC CGT GCC AAA GAA G & \multirow{2}{*}{ This study } \\
\hline VTA3seq 6 & ACG GGA ACC ATA GTG ATC CA & \\
\hline \multicolumn{3}{|l|}{ For RT-PCR } \\
\hline$q A B A A-F$ & CGA GAA GAA CCT CAA CAA GGA G & \multirow{2}{*}{ This study } \\
\hline$q A B A A-R$ & CTA CGG TGA AGA GAC GGG AAA C & \\
\hline qALG9-F & TTG CCG TTT TCA CTC AAC AA & \multirow{2}{*}{ This study } \\
\hline qALG9-R & CCA AAG CCA CTA TCC GTG AC & \\
\hline qCAP20-F & TAT CTC CAG AAG GCC GAC AC & \multirow{2}{*}{ This study } \\
\hline qCAP20-R & GCC ATT GCC AAG TTT CTT GT & \\
\hline qCON-6-F & CGA GGA GAG CAA GCA GCA CT & \multirow{2}{*}{ This study } \\
\hline qCON-6-R & GAC GTT GCC AGG GTT CTT GT & \\
\hline qCON-8-F & ACG CAG CTC CAA CAC CCT CT & \multirow{2}{*}{ This study } \\
\hline qCON-8-R & GTG AAA TTG CGC CAC ATC TTG & \\
\hline qCPX2-F & ATG TCG CCT CGC TGA AGA AGA & \multirow{2}{*}{ This study } \\
\hline $\mathrm{qCPX2-R}$ & ACG CTT GTC TGA GTT GCG GTA G & \\
\hline $\mathrm{qFAS1-F}$ & CTG CTG ACC AAC GAG ACG TA & \multirow{2}{*}{ This study } \\
\hline $\mathrm{qFAS1-R}$ & GCT AAT GTT GAG GGG CAG AG & \\
\hline
\end{tabular}


Table 1. (Continued)

q: RT-PCR primers, F: forward primer, R: reverse primer, restriction sites and overhang were underlined.

\begin{tabular}{|c|c|c|}
\hline Primer & 5'-3' direction & Reference \\
\hline qGAPDH-F & AAC GTC TCC GTT GTT GAC CTG A & \multirow{2}{*}{ This study } \\
\hline qGAPDH-R & GTC CTC GGT GTA GGC CAG AAT G & \\
\hline q/N01-F & GAC ACC AAG ACC AGG AAG GA & \multirow{2}{*}{ This study } \\
\hline$q / N 01-R$ & GAA GGC AGA GGC ACA ATC TG & \\
\hline qMAD1-F & CGA CCT CGA GTT CCA CTA CG & \multirow{2}{*}{ This study } \\
\hline $\mathrm{qMAD1-R}$ & CGA AGC CGA TCT TCC AGA TA & \\
\hline qMAD2-F & GGA GGA TGA TGA CTG GCA CT & \multirow{2}{*}{ This study } \\
\hline qMAD2-R & GGC AGA TAG TGG TGG TCT GG & \\
\hline $\mathrm{qNLP2-F}$ & CCG TCT CTC ATC AGC ATC GT & \multirow{2}{*}{ This study } \\
\hline $\mathrm{qNLP2-R}$ & CGT TGA ACA CCT TGA GGT ACG & \\
\hline qOLG 70 & CAG CGA AAC GCG ATA TGT AG & \multirow{2}{*}{$\begin{array}{c}\text { Eynck et al. } \\
(2007)\end{array}$} \\
\hline qOLG 71 & GGC TTG TAG GGG GTT TAG A & \\
\hline $\mathrm{qPLS1-F}$ & GGC TGC GAG AAA CTT GAT CT & \multirow{2}{*}{ This study } \\
\hline $\mathrm{qPLS1-R}$ & AGG TAG ACA CCC ACG CAA AG & \\
\hline $\mathrm{qPP} 2 A 1-\mathrm{F}$ & CCC GAG AAG GAG GTT GAA GT & \multirow{2}{*}{ This study } \\
\hline $\mathrm{qPP} 2 A 1-\mathrm{R}$ & CAT CCG TCT CCT TAC GCA TT & \\
\hline $\mathrm{q} P P 2 A 2-\mathrm{F}$ & ACA TTC GCT TCA ACG TAG CC & \multirow{2}{*}{ This study } \\
\hline $\mathrm{qPP} 2 A 2-\mathrm{R}$ & AAC ATC CAC ATC GTC GTC CT & \\
\hline $\mathrm{q} P R Y 1-\mathrm{F}$ & GCT GCC ATT CTC ACA ACA CA & \multirow{2}{*}{ This study } \\
\hline $\mathrm{q} P R Y 1-\mathrm{R}$ & TCC AAT GGC CAG ATT CTC TC & \\
\hline qScFL011-F & GGT GTC ACT GGT CCA AAA GG & \multirow{2}{*}{ This study } \\
\hline qScFL011-R & TTG CAT ATT GAG CGG CAC TA & \\
\hline qScFL01-F & GAA CGC TGT TTC TTG GGG TA & \multirow{2}{*}{ This study } \\
\hline qScFLO1-R & TGA AAG TAC CGG TCC ATG GTT & \\
\hline qScFLO8-F & CAG CAG CCT TTG CTC AAG AT & \multirow{2}{*}{ This study } \\
\hline qScFLO8-R & CTC TGA GCC ACC TCT GGA AG & \\
\hline qSFL1-F & CGA ATC GCT ACA CGA CTT GA & \multirow{2}{*}{ This study } \\
\hline qSFL1-R & TTA GCG TCG TTG CTG CTA TG & \\
\hline
\end{tabular}


Table 1. (Continued)

q: RT-PCR primers, F: forward primer, R: reverse primer, restriction sites and overhang were underlined.

\begin{tabular}{|c|c|c|}
\hline Primer & 5'-3' direction & Reference \\
\hline qSNOD1-F & CCC AAA AGC AGG TCA AGA AG & \multirow{2}{*}{ This study } \\
\hline qSNOD1-R & ATG GCG AGG ACA TTG ATG GT & \\
\hline qSOD3-F & GAA AAC ACG GCT TCG TTG AGT C & \multirow{2}{*}{ This study } \\
\hline qSOD3-R & GAG GTT GCT GCT GAA GTG AAG G & \\
\hline qSOM1-F & CCA ACA AGC AGA TGC CTA ATG C & This study \\
\hline qSOM1-R & CTT GCA GCG TGG TTA CTT C & This study \\
\hline qSOMA-F & AAT GCA GTT GAT GCT GCT TG & This study \\
\hline qSOMA-R & CCT CGT TTC ATC TGC TCG TT & This study \\
\hline qTAF10-F & CCA GGA TCA GGT CTT CCG TA & This study \\
\hline qTAF10-R & TCA GCA ACA GCG CTA CTG AG & This study \\
\hline qVDH1-F & ACG ATT GCT CTG TTT GCT GGA G & This study \\
\hline$q V D H 1-R$ & CCT GGC ACT CTT TGG GGT AGA & This study \\
\hline qVTA1-F & CAC AGG GGC GAG TCT AGG TA & This study \\
\hline qVTA1-R & CCC GAG GTA CCC GAT CAT AG & This study \\
\hline qVTA2-F & TAC TCC TTC GTT CCG ATT CCT G & This study \\
\hline qVTA2-R & GCG CAT TGA GAT GGT TCA GAG T & This study \\
\hline qVTA3-F: & GGA TGG CAA AGT CAA CGT CT & This study \\
\hline qVTA3-R: & CGA ACA GAC CGA ATT GAT CC & This study \\
\hline qVTA4-F & ATC CCA AGC TCC AGG CTA CT & This study \\
\hline qVTA4-R & GTA CGA GGC GGT CTG TGA AG & This study \\
\hline qVTA5-F & CCC CTC ATC CCA GTC TCA AT & This study \\
\hline qVTA5-R & CAA CGC TCG TGG AAC TCT TC & This study \\
\hline qVTA6-F & CAA GAA GTT CTC CCG CTC TG & This study \\
\hline qVTA6-R & GAG AGA CGT TGG GCG AAG AC & This study \\
\hline
\end{tabular}




\subsubsection{Plasmids}

Plasmids used in this study are listed in Table 2.

Table 2. Plasmids used in this study

p: promoter, t: terminator, R: resistance, NAT: nourseothricin, $H P H$ : hygromycin.

\begin{tabular}{|c|c|c|}
\hline Plasmid & Description & Reference \\
\hline pJET1.2/blunt & Cloning vector with blunt ends & (Fermentas) \\
\hline pME4548 & pgpdA::NAT1R & This study \\
\hline pME4549 & pSOM1::pgpdA::NAT1R::SOM1t & This study \\
\hline pPK2 & pgpdA::HPHR::trpC $C^{t}$ & Covert (2001) \\
\hline pME4550 & pSOM1:SOM1::SOM1t, pgpdA:::HPH::trpC ${ }^{t}$ & This study \\
\hline pGreen2 & pgpdA::GFP::trpC ${ }^{t}$ & Tran (2014) \\
\hline pME4551 & pSOM1::SOM1::GFP::trpCt & This study \\
\hline pME4552 & pgpdA::ScFLO8::GFP:::trpC ${ }^{t}$ & This study \\
\hline pME4553 & pgpdA::AfSOMA::GFP::trpC ${ }^{t}$ & This study \\
\hline pME4554 & pVTA3::pgpdA::NAT1R::VTA3t & This study \\
\hline pME4555 & pVTA3:VTA3::VTA3, ${ }^{\text {pgpdA:::HPHR:::trpC }}{ }^{t}$ & This study \\
\hline pME4556 & pVTA3::VTA3::GFP::trpC ${ }^{t}$ & This study \\
\hline pME4557 & pgpdA::SOM1::GFP::trpC ${ }^{t}$ & This study \\
\hline pME2795 & pGAL1, CYC1t, URA3, Amp ${ }^{R}$ & Mumberg (1994) \\
\hline pME4558 & PGAL1::ScFLO8:: CYC1t, URA3, Amp ${ }^{R}$ & This study \\
\hline pME4559 & pGAL1::SOM1:: CYC1t, URA3, Amp ${ }^{R}$ & This study \\
\hline pME2787 & pMET25, CYC1t, URA3, Amp ${ }^{R}$ & Mumberg (1994) \\
\hline pME4560 & pMET25::ScFLO8::CYC1t, URA3, Amp ${ }^{R}$ & This study \\
\hline pME4561 & pMET25::SOM1::CYC1t, URA3, Amp & This study \\
\hline pME4561 & pGAL1::VTA3::CYC1t, URA3, Amp ${ }^{R}$ & This study \\
\hline pNAT1 & pgpdA::NAT1 & Janus (2007) \\
\hline
\end{tabular}




\subsubsection{Organisms}

\subsubsection{Bacterial strains}

Escherichia coli strain DH5a (Invitrogen, Karlsruhe, Germany) and Agrobacterium tumefaciens strain AGL1 (Lazo et al., 1991) were used for transformation procedures in this study. They were cultivated in Luria-Bertani (LB) medium $(0.5 \%$ yeast extract, $1 \%$ bacto-tryptone and $1 \% \mathrm{NaCl})$ at $37^{\circ} \mathrm{C}$ for $E$. coli or $25^{\circ} \mathrm{C}$ for $A$. tumefaciens.

\subsubsection{Fungal strains}

Saccharomyces cerevisiae strains are derived from BY4741 and BY4742, the non-adhesive S288c genetic background, carrying a truncated FLO8 gene. The S288c background has a nonsense mutation in the open reading frame (ORF) of the FLO8 gene encoding a transcriptional regulator of FLO1 and FLO11 adherent genes (Liu et al., 1996). Therefore, the expression of FLO1, as well as FLO11, is blocked. All S. cerevisiae strains were incubated in YPD or SC-Ura medium at $30^{\circ} \mathrm{C}$.

Verticillium dahliae strain JR2 wild-type was provided by Bart Thomma, Laboratory of Phytopathology in Wageningen, Netherlands (Fradin et al., 2009). All strains were inoculated at $25^{\circ} \mathrm{C}$ in potato dextrose broth (PDA) (Sigma-Aldrich Chemie $\mathrm{GmbH}$, Munich, Germany), Minimal medium (MM) (Bennett \& Lasure, 1991), Czapek-Dox medium (CDM) (Smith, 1948) or a modified simulated xylem medium (SXM) (Neumann \& Dobinson, 2003) which is composed of $0.2 \%$ pectin from citrus peel (Sigma-Aldrich Chemie $\mathrm{GmbH}$, Munich, Germany), $0.4 \%$ casein hydrolysate (OXOID Ltd, Basingstoke, Hampshire, England), $2 \mathrm{mM} \mathrm{MgSO}_{4}, 1 \times$ ASPA, and $1 \mathrm{x}$ trace elements.

The Verticillium strains were grown in SXM on a shaker at $120 \mathrm{rpm}$ at $25^{\circ} \mathrm{C}$ for seven days. Conidia were harvested by filtration of the culture through a miracloth membrane (Calbiochem, Darmstadt, Germany), the filtrate was washed twice with sterile water before resuspending in the solution containing $0.96 \% \mathrm{NaCl}$ and $0.05 \%$ Tween 80 . The number of spores was counted in a counting chamber under a binocular microscope and the spore density was adjusted to $10^{7}$ spores $/ \mathrm{ml}$. Aliquots of spore suspension containing $25 \%$ of glycerol were frozen in liquid nitrogen and stored at $-80^{\circ} \mathrm{C}$. Fungal strains used in this study are listed in Table 3. 
Table 3. Fungal strains used in this study

Amp: Ampicillin, p: promoter, t: terminator, R: resistance, NAT: nourseothricin, HPH: hygromycin.

\begin{tabular}{|c|c|c|}
\hline Strain & Description & Reference \\
\hline \multicolumn{3}{|l|}{ Verticillium } \\
\hline VGB0001 & $\triangle S O M 1:: N A T 1 R$ & This study \\
\hline VGB0002 & $\triangle S O M 1:: N A T 1^{R}$ & This study \\
\hline VGB0003 & 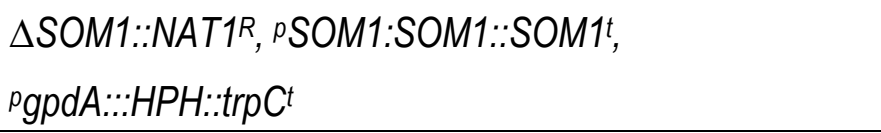 & This study \\
\hline VGB0004 & $\begin{array}{l}\triangle S O M 1:: N A T 1 R, \text { pSOM1:SOM1::SOM1t, } \\
\text { pgpdA:::HPH::trpC }{ }^{t}\end{array}$ & This study \\
\hline VGB0009 & $\triangle V T A 3:: N A T 1^{R}$ & This study \\
\hline \multirow[t]{2}{*}{ VGB0010 } & $\triangle V T A 3:: N A T 1^{R}$ & This study \\
\hline & JR2-wt & Fradin et al. (2009) \\
\hline VGB0074 & $\triangle S O M 1:: N A T 1 R$, pgpdA:GFP::trpC ${ }^{t}$, pgpdA:.:.HPH::trpCt & This study \\
\hline VGB0075 & $\triangle S O M 1:: N A T 1^{R},{ }^{p} g p d A:: G F P::$ trpC $^{t},{ }^{p} g p d A::: H P H:: \operatorname{trp} C^{t}$ & This study \\
\hline VGB0079 & $\triangle V T A 3:: N A T 1 R, p V T A 3: V T A 3:: V T A 3^{t}$, pgpdA:::HPH:::trpC ${ }^{t}$ & This study \\
\hline VGB0080 & $\triangle V T A 3:: N A T 1 R, p V T A 3: V T A 3:: V T A 3 t$, pgpdA:::HPH:::trpC ${ }^{t}$ & This study \\
\hline VGB0084 & $\begin{array}{l}\triangle S O M 1:: N A T 1 R \text {, pSOM1::SOM1::GFP::trpC }{ }^{t} \\
\text { pgpdA:::HPH::trpC }\end{array}$ & This study \\
\hline VGB0085 & $\begin{array}{l}\triangle S O M 1:: N A T 1^{R}, \text { pSOM1::SOM1::GFP::trpC }{ }^{t} \\
\operatorname{pgpdA:::HPH::trpC} C^{t}\end{array}$ & This study \\
\hline VGB0090 & JR2, pgpdA::GFP::trpC ${ }^{t}$, pgpdA::.:HPH::trpC ${ }^{t}$ & This study \\
\hline VGB0172 & 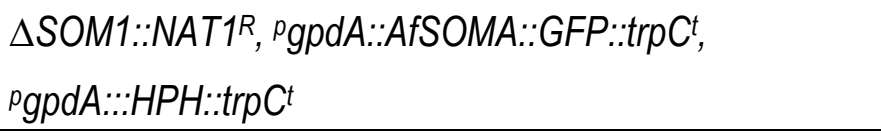 & This study \\
\hline VGB0173 & $\begin{array}{l}\triangle S O M 1:: N A T 1 R, \text { pgpdA::AfSOMA::GFP::trpC }{ }^{t}, \\
\text { pgpdA:::HPH::trpC }{ }^{t}\end{array}$ & This study \\
\hline
\end{tabular}


Table 3. (Continued)

Amp: Ampicillin, p: promoter, t: terminator, R: resistance, NAT: nourseothricin, HPH: hygromycin.

\begin{tabular}{|c|c|c|}
\hline Strain & Description & Reference \\
\hline VGB0174 & JR2, pgpdA::SOM1::GFP::trpC $C^{t}$, pgpdA:::HPH::trpC ${ }^{t}$ & This study \\
\hline VGB0175 & JR2, pgpdA::SOM1::GFP::trpC $C^{t}$, pgpdA:::HPH::trpC ${ }^{t}$ & This study \\
\hline VGB0178 & $\triangle S O M 1:: N A T 1 R$, pgpdA::ScFLO8::GFP:::trpC', pgpdA:::HPH::trpCt & This study \\
\hline VGB0179 & $\triangle S O M 1:: N A T 1^{R},{ }^{p} g p d A:: S c F L O 8:: G F P::$ trpC ${ }^{t},{ }^{p} g p d A::: H P H:: t r p C^{t}$ & This study \\
\hline VGB0184 & $\triangle V T A 3:: N A T 1^{R}$, pgpdA:GFP::trpC ${ }^{t}$, pgpdA:::HPH::trpC ${ }^{t}$ & This study \\
\hline VGB0185 & $\triangle V T A 3:: N A T 1^{R}$, pgpdA:GFP::trpC ${ }^{t}$, pgpdA:::HPH::trpC ${ }^{t}$ & This study \\
\hline VGB0281 & $\triangle V T A 3::$ NAT1R, pVTA3::VTA3::GFP::trpC', pgpdA:::HPH::trpC ${ }^{t}$ & This study \\
\hline VGB0282 & $\triangle V T A 3:: N A T 1 R, p V T A 3:: V T A 3:: G F P:: \operatorname{trpC} C^{t}$, pgpdA:::HPH::trpC ${ }^{t}$ & This study \\
\hline \multicolumn{3}{|c|}{ S. cerevisiae } \\
\hline BY4742 & MATa, $\triangle F L O 8, \triangle H I S 3, \triangle L E U 2, \triangle U R A 3$ & Euroscarf \\
\hline BY4741 & MATa, $\triangle F L O 8, \Delta H I S 3, \Delta L E U 2, \triangle M E T 25, \triangle U R A 3$ & Euroscarf \\
\hline $\mathrm{RH} 3647$ & BY4742, pMET25, CYC1t, URA3, Amp ${ }^{R}$ & This study \\
\hline RH3648 & BY4741, pGAL1, CYC1t, URA3, Amp $R$ & This study \\
\hline RH3649 & BY4742, pMET25::ScFLO8::CYC1t, URA3, Amp ${ }^{R}$ & This study \\
\hline $\mathrm{RH} 3650$ & BY4741, pGAL1::ScFLO8::CYC1t, URA3, Amp ${ }^{R}$ & This study \\
\hline $\mathrm{RH} 3651$ & BY4742, pMET25::SOM1::CYC1t, URA3, Amp ${ }^{R}$ & This study \\
\hline $\mathrm{RH} 3652$ & BY4741, pGAL1::SOM1::CYC1t, URA3, Amp R & This study \\
\hline RH3653 & BY4741, pGAL1::VTA3::CYC1t, URA3, Amp ${ }^{R}$ & This study \\
\hline
\end{tabular}




\subsection{Methods}

\subsubsection{Bioinformatic analysis}

Predicted functions of deduced proteins and conserved domains/motifs were examined with InterProScan (Mulder \& Apweiler, 2007), Pfam (Finn et al., 2016), and BLAST searches at the National Center for Biotechnology Information (http://www.ncbi.nlm.nih.gov) (Mittler et al., 2010). Nuclear localisation signals (NLS) were predicted at SeqNLS: Nuclear localisation signal prediction based on frequent pattern mining and linear motif scoring (http://mleg.cse.sc.edu/seqNLS/) (Lin \& Hu, 2013). Gene numbers and gene sequences of $V$. dahlia are according to the VertiBase (http://www.vertibase.org). Protein alignments were performed by Clustal Omega at European Molecular Biology Laboratory-European Bioinformatics Institute (http://www.ebi.ac.uk) (Sievers \& Higgins, 2014).

\subsubsection{Gene deletion, complementation, and overexpression}

\subsubsection{Gene deletion}

The vector pPK2 (Covert et al., 2001) was digested with enzymes Sacl and Apal. In parallel, the nourseothricin resistance cassette $(1.45 \mathrm{~kb})$ was amplified by PCR from the pNAT1 vector (Janus et al., 2007) with the primer pair gdpA-NAT-F/ gdpA-NAT-R (Table 1). This cassette was digested with Sacl and Apal and ligated into pPK2 to generate the pME4548 vector (Table 2). 


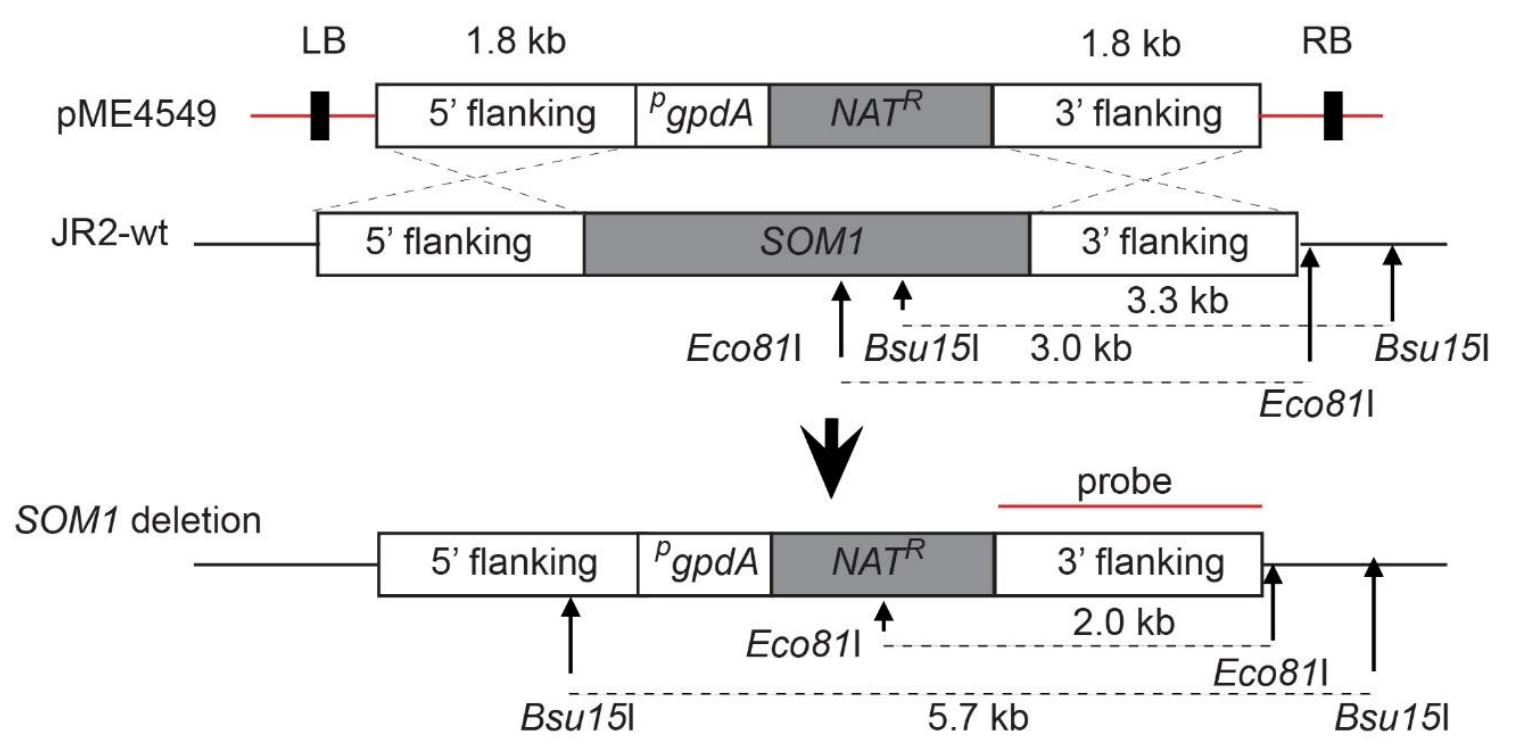

Figure 12. Strategies of SOM1 deletion and confirmation in V. dahliae. SOM1 was knocked out by using a strategy via homologous recombination between the deletion construct and wild-type gene locus. 5' and 3' flanking region (FR) are around $1.8 \mathrm{~kb}$; p: promoter; R: resistance. The restriction enzyme site and predicted sizes are indicated.

Fragments of $1.9 \mathrm{~kb}$ upstream and downstream of SOM1 (Figure 12) or the $1.1 \mathrm{~kb}$ of VTA3 (Figure 13) were amplified by Phusion ${ }^{\mathrm{TM}}$ high-fidelity DNA polymerase (Thermo Fisher Scientific $\mathrm{GmbH}$, St. Leon-Rot, Germany) with primer pairs SOM1-P1/SOM1-P2, SOM1-P3/SOM1-P4, VTA3-P1/VTA3-P2, or VTA3-P3/ VTA3-P4 (Table 1). The fragments were processed by restriction enzymes (Table 1). Finally, they were individually cloned into pME4548 digested with the same enzymes to produce pME4549 and pME4554 (Figure 12, 13, Table 2). The results were confirmed by PCR and digestion with restriction enzymes before introducing into JR2-wt strain via A. tumefaciens AGL-1 strain (Lazo et al., 1991; Timpner et al., 2013) to generate VGB0001, VGB0002, VGB0009, and VGB0010 (Table 3). The fungal transformants were selected on potato dextrose agar (PDA) containing nourseothricin $(50 \mu \mathrm{g} / \mathrm{ml})$ and cefotaxime $(100 \mu \mathrm{g} / \mathrm{ml})$. The deletion strains were confirmed by Southern hybridization. 


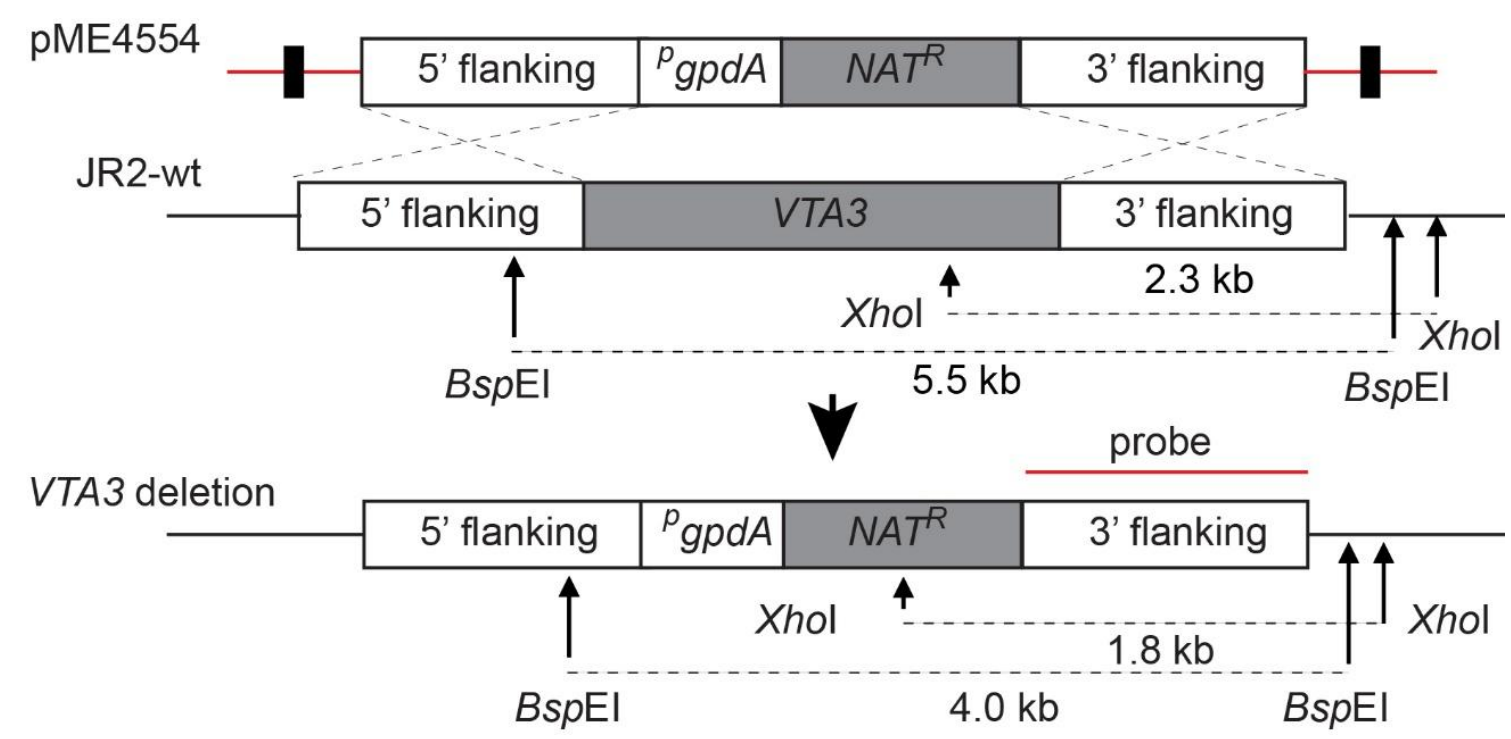

Figure 13. Deletion and confirmation strategies of VTA3 gene in V. dahliae. VTA3 was knocked-out via homologous recombination between the deletion construct and wild-type gene locus. 5' and 3' flanking region are around $1.0 \mathrm{~kb}$; p: promoter; R: resistance. The restriction enzyme site and predicted sizes are indicated.

\subsubsection{Gene complementation}

For complementation, the fragments including $1.2 \mathrm{~kb}$ upstream, the ORF and $0.8 \mathrm{~kb}$ downstream of SOM1 and VTA3 were amplified from genomic DNA of JR2-wt using the primer pairs SOM1comp-F/SOM1comp-R or VTA3comp-F/VTA3comp-R (Table 1). They were individually cloned into pPK2 (Covert et al., 2001) between $X$ bal and Sbfl to generate pME4550 and pME4555 (Figure 14, Table 2). This construct was transformed into the VGB0001 or VGB0009 strain, respectively, via $A$. tumefaciens to generate VGB0003, VGB0004, VGB0079, and VGB0080 (Table 3). The transformants were selected on PDA plates containing both, hygromycin $(50 \mu \mathrm{g} / \mathrm{ml})$, and nourseothricin $(50 \mu \mathrm{g} / \mathrm{ml})$ and confirmed by Southern hybridization. 
(a)
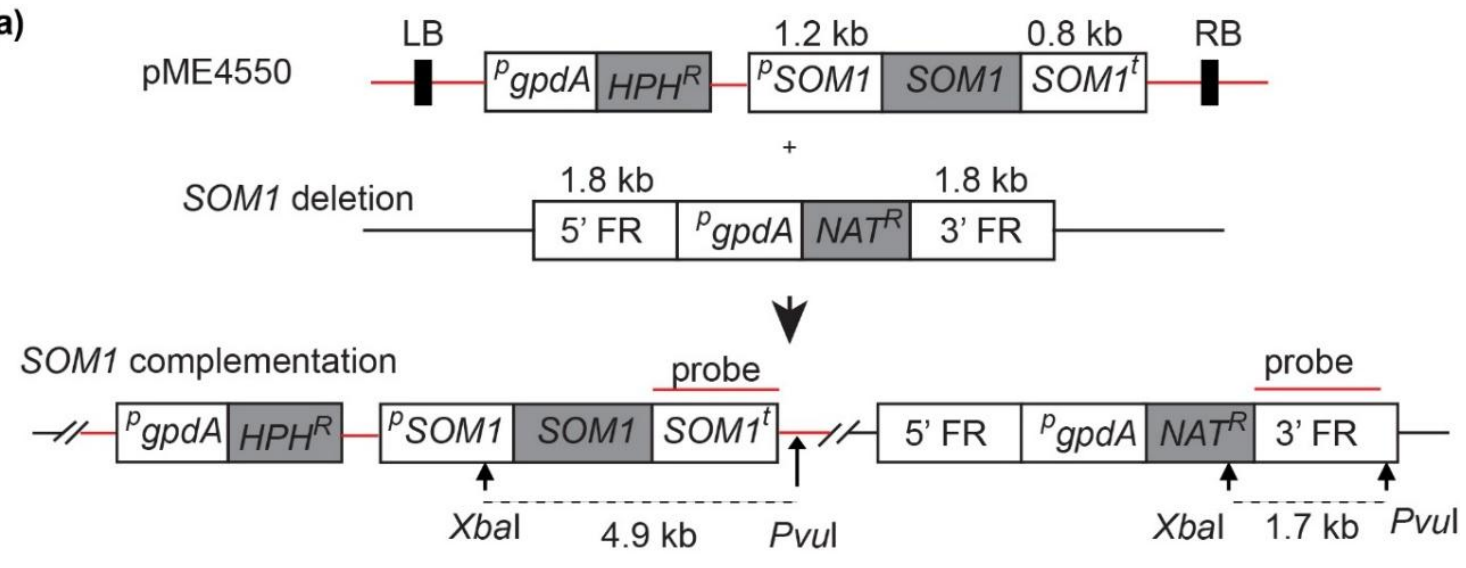

(b)

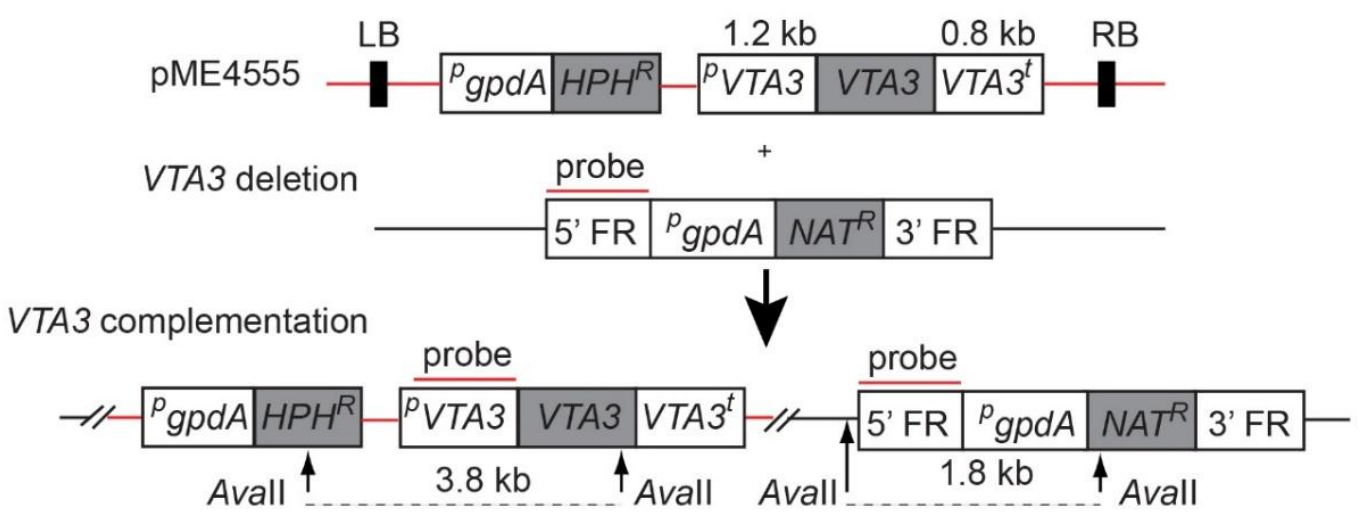

Figure 14. Complementation of SOM1 and VTA3 in V. dahliae and confirmation strategies. The construct for creating complementation strains contains the selection marker of hygromycin under the supervision of the gpdA promoter and a gene under its native promoter $(1.2 \mathrm{~kb})$ and the native terminator $(0.8 \mathrm{~kb})$. This construct was ectopically transformed into the deletion strain to generate the complementation strains. (a) Complementation of SOM1 and verification strategies. (b) Complementation of VTA3 and confirmation strategies. $\mathrm{p}$ : promoter; R: resistance. The name of strains, restriction enzyme site, and predicted sizes are indicated.

\subsubsection{Gene overexpression}

The ORF of SOM1 without the stop codon was fused to the N-terminal end of the GFP gene in the pGreen2 backbone between Xhol and Kpnl to generate the pME4557 plasmid (Table 2). This combination gene is under the control of gpdA promoter. This construct was transformed into the $V$. dahliae JR2 wild-type to create VGB0174 and VGB0175 (Table 3) via A. tumefaciens (Timpner et al., 2013). The transformed strains were selected on PDA plates and under a fluorescence microscope. The SOM1 transcript levels was examined by RT- PCR. The growth, conidiation, and microsclerotia formation were tested. 


\subsubsection{Genetic manipulations}

\subsubsection{E. coli transformation}

Preparation of E. coli competent cells using DMSO and TB buffer and transformation methods were modified from (Inoue et al., 1990).

Preparation of E. coli competent cells: One fresh colony of $E$. coli was incubated in $5 \mathrm{ml} \mathrm{LB}$ medium ( $0.5 \%$ yeast extract, $1 \%$ bacto-tryptone, and $1 \% \mathrm{NaCl}$ ) at $37^{\circ} \mathrm{C}$ on a rotator overnight. $500 \mu \mathrm{l}$ of overnight cultures was added into $5 \mathrm{ml}$ LB and grown for 4-5 $\mathrm{h}$ in the same condition as above. $1 \mathrm{ml}$ of this culture was added to $250 \mathrm{ml} \mathrm{SOB}$ medium ( $2 \%$ tryptone, $0.5 \%$ yeast extract, $10 \mathrm{mM} \mathrm{NaCl}, 2.5 \mathrm{mM} \mathrm{KCl}$, $10 \mathrm{mM} \mathrm{MgCl}_{2}$, and $10 \mathrm{mM} \mathrm{MgSO}_{4}$ ) in a one liter flask and incubated on a shaker at $120 \mathrm{rpm}$ at $20^{\circ} \mathrm{C}$ overnight until the $\mathrm{OD}_{600}$ of culture is around 0.6. The culture was kept in ice for $10 \mathrm{~min}$ and centrifuged at $2500 \mathrm{rpm}$ for $10 \mathrm{~min}$ at $4^{\circ} \mathrm{C}$. The pellet was washed with $80 \mathrm{ml}$ of cool TB buffer (10 mM HEPES, $15 \mathrm{mM} \mathrm{CaCl}_{2} .2 \mathrm{H}_{2} \mathrm{O}, 250 \mathrm{mM}$ $\mathrm{KCl}, 55 \mathrm{mM} \mathrm{MnCl}_{2}, \mathrm{pH}$ 6.7) for $10 \mathrm{~min}$. The pellet was collected by centrifugation at the same condition as above and resuspended in $20 \mathrm{ml}$ of TB buffer. DMSO was slowly added to suspension by gently swirling to a final concentration of $7 \%$. This mixture was incubated for $10 \mathrm{~min}$ on ice before distributing $400 \mu$ l aliquot into $1.5 \mathrm{ml}$ eppendorf tubes, frozen in liquid nitrogen and stored at $-80^{\circ} \mathrm{C}$.

E. coli transformation: $10 \mu \mathrm{l}$ of a ligation or $50 \mathrm{ng}$ of a plasmid was added into an eppendorf tube containing $200 \mu$ of thawed competent cells. This tube was kept on ice for $20 \mathrm{~min}$ and at $42^{\circ} \mathrm{C}$ on a heating block for $40 \mathrm{~s} .800 \mu \mathrm{l}$ of SOC medium, SOB addition $20 \mathrm{mM}$ glucose, was added into suspension. This mixture was incubated at $37^{\circ} \mathrm{C}$ on a shaker for $1 \mathrm{~h}$. The cell pellet was collected by centrifugation at $10000 \mathrm{rpm}$ for $1 \mathrm{~min}$ and spread on LB agar plates containing an appropriate antibiotic for selection (ampicillin or kanamycin $100 \mu \mathrm{g} / \mathrm{ml}$ ). The positive colonies were confirmed by colony PCR with specific primer pairs and digested with suitable restriction enzymes. 


\subsubsection{A. tumefaciens transformation}

Preparation of $A$. tumefaciens competent cells and transformation were modified from (Jyothishwaran et al., 2007).

Preparation of competent cells: A. tumefaciens cells were grown in $100 \mathrm{ml}$ LB medium containing $50 \mu \mathrm{g} / \mathrm{ml}$ carbercillin in a one liter flask at $25^{\circ} \mathrm{C}$ on a shaker overnight until the $\mathrm{OD}_{600}$ is around 0.6. Overnight cultures were kept on ice for $15 \mathrm{~min}$ and cell pellets were collected by centrifugation at $5000 \mathrm{rpm}$ for $10 \mathrm{~min}$ at $4^{\circ} \mathrm{C}$. Pellets were sequentially washed with $20 \mathrm{ml} \mathrm{MgCl}_{2} 100 \mathrm{mM}$ for $1 \mathrm{~h}$ and dissolved with $20 \mathrm{ml} \mathrm{CaCl}_{2} 20 \mathrm{mM}$ for $4 \mathrm{~h}$. Glycerol was added into resuspension to a final concentration of $25 \%$. The mixture was distributed $200 \mu \mathrm{l}$ aliquot to $1.5 \mathrm{ml}$ eppendorf tubes, frozen in liquid nitrogen and store at $-80^{\circ} \mathrm{C}$.

A. tumefaciens transformation: $1000 \mathrm{ng}$ of a binary vector was added into an eppendorf tube containing $200 \mu$ of thawed competent cells. The tube was incubated $10 \mathrm{~min}$ on ice, $10 \mathrm{~min}$ in liquid nitrogen, and $10 \mathrm{~min}$ at $37^{\circ} \mathrm{C}$ in a heating block. The tube was added with $800 \mu$ of SOC medium and incubate at $28^{\circ} \mathrm{C}$ on a shaker at $120 \mathrm{rpm}$ for one hour. Cell pellets were collected by centrifugation at $13000 \mathrm{rpm}$ for $1 \mathrm{~min}$ and spread on LB plates containing $100 \mu \mathrm{g} / \mathrm{ml} \mathrm{kanamycin}$. Plates were grown at $25^{\circ} \mathrm{C}$ for $72 \mathrm{~h}$ to gain colonies. Positive colonies were confirmed by PCR reaction with specific primer pairs.

\subsubsection{S. cerevisiae transformation}

The ORFs of SOM1 and VTA3 were cloned into pME2795 or pME2787 (Mumberg et al., 1994) plasmids between Xhol and Hindll to create pME4559, pME4561, and pME4562 (Table 2). The recombinant plasmids were verified by PCR and enzymatic digestion and transformed into a non-adhesive yeast strain BY4741 or BY4742 to generate $\mathrm{RH} 3651, \mathrm{RH} 3652$, and $\mathrm{RH} 3653$ strains (Table 3). The yeast transformation was performed following the lithium acetate/singlestranded carrier DNA/polyethylene glycol (LiAc/SS carrier DNA/PEG) method (Gietz \& Schiestl, 2007). Adhesion and flocculation tests were previously described (Tran et al., 2014; Lin et al., 2015). 


\subsubsection{V. dahliae transformation}

Verticillium transformation method was modified from (Timpner et al., 2013; Tran et al., 2014). A positive colony of $A$. tumefaciens was grown in $5 \mathrm{ml}$ of LB medium containing appropriate antibiotic overnight. $500 \mu$ of overnight cultures were added to $5 \mathrm{ml}$ of induction medium including acetosyringone (IMAS) (1X MM salts, $0.5 \%$ glycerol and $10 \mathrm{mM}$ glucose) and grown at $25^{\circ} \mathrm{C}$ for $5 \mathrm{~h}$ on a shaker at $120 \mathrm{rpm}$. This culture was mixed with the same volume of the spore solution $\left(10^{6} \mathrm{spores} / \mathrm{ml}\right)$. This mixture was spread onto the filter paper (Sartorius, Göttingen, Germany) on an agar plate of the IMAS medium that is similar to liquid medium excepting to contain $5 \mathrm{mM}$ glucose instead of $10 \mathrm{mM}$. The plate was co-cultivated in the dark at $25^{\circ} \mathrm{C}$ for $72 \mathrm{~h}$. The filter paper was transferred to a PDA (potato dextrose agar) plate supplied with suitable antibiotics to select transformation and cefotaxime to kill all agrobacteria. Deletion strains were selected by nourseothricin $(50 \mu \mathrm{g} / \mathrm{ml})$ and complementation by hygromycin $(50 \mu \mathrm{g} / \mathrm{ml})$. The plate was grown for two weeks. Colonies were separately cultivated on selection medium and confirmed by Southern hybridization.

\subsubsection{Confirmation of transformation}

\subsubsection{DNA purification}

Plasmid purification from E. coli: A single colony of E. coli containing a plasmid of interest was incubated in $5 \mathrm{ml}$ LB medium supplemented suitable antibiotic on a rotator at $37^{\circ} \mathrm{C}$ overnight. The plasmid was isolated using Plasmid Miniprep Kits (Qiagen, Hilden, Germany) according to the manufacturer's protocol.

DNA isolation from yeast: Total genomic DNA isolation method was modified from (Amberg et al., 2005). The yeast strain was grown in $10 \mathrm{ml}$ of YEP medium ( $1 \%$ yeast extract, $2 \%$ peptone, and $2 \%$ glucose) at $30^{\circ} \mathrm{C}$ on a rotatory shaker overnight. The cell pellet was collected by centrifuging at $3000 \mathrm{rpm}$ for $10 \mathrm{~min}$. The pellet was ground to fine powder by a Mixer Mill MM 400 (Retsch $\mathrm{GmbH}$, Haan, Germany) using two small marble in a $2 \mathrm{ml}$ centrifugation tube in liquid nitrogen. $300 \mu \mathrm{l}$ of Smash-Grab lysis buffer (10 mM Tris pH 8.0, $1 \mathrm{mM}$ EDTA, $100 \mathrm{mM} \mathrm{NaCl}, 1 \%$ SDS, $2 \%$ Triton $\mathrm{X}-100$ ) was added to a fine powder and mixed well by vortexing for $2 \mathrm{~min} .300 \mu \mathrm{l}$ of phenol/chloroform was added to the tube and inverted 10 times. The tube was centrifuged at $13000 \mathrm{rpm}$ for $10 \mathrm{~min}$. 
The supernatant was transferred to a new $1.5 \mathrm{ml}$ tube containing the same volume of isopropanol and kept in -20 for 30 min. The pellet of DNA was harvested by centrifugation at $13000 \mathrm{rpm}$ for $10 \mathrm{~min}$ and washed twice with ethanol $75 \%$. The pellet was dried at $37^{\circ} \mathrm{C}$ for $30 \mathrm{~min}$ and dissolved in $100 \mu \mathrm{l}$ TE buffer $(10 \mathrm{mM}$ Tris $\mathrm{pH} 8.0$ and $1 \mathrm{mM}$ EDTA). The RNA was eliminated by $4 \mu \mathrm{RNase}(10 \mathrm{mg} / \mathrm{ml})$ at $65^{\circ} \mathrm{C}$ for $30 \mathrm{~min}$. The quality of isolated DNA was checked by running a gel and the concentration of DNA was measured by a Nanodrop ND 1000 spectrophotometer (Peqlab Biotechnologies GmbH, Erlangen, Germany).

DNA isolation from Verticillium: Fungal spores (2 $\mathrm{ml}$ of $10^{7} \mathrm{spores} / \mathrm{ml}$ ) were added into $50 \mathrm{ml} \mathrm{PDB}$ (potato dextrose broth) medium and grown for seven days. Mycelia were harvested by filtering with a miracloth membrane (Calbiochem, Darmstadt, Germany) and ground to fine powder in liquid nitrogen using a mortar and pestle. The fungal powder was used directly for genomic DNA extraction. The extraction method was modified from (Kolar et al., 1988). About $1.0 \mathrm{~g}$ of fungal powder was transferred into $2 \mathrm{ml}$ centrifugation tube. One milliliter of pre-warmed lysis buffer (50 mM Tris pH 7.2, 50 mM EDTA, 3\% SDS, and 1\% 2-Mercaptoethanol) was added into fungal powder, mixed at maximum speed for 30 seconds, and incubated at $65^{\circ} \mathrm{C}$ for $1 \mathrm{~h}$. The mixture was centrifuged at maximum speed for $5 \mathrm{~min} .800 \mu \mathrm{l}$ of the supernatant was transferred to a new $2.0 \mathrm{ml}$ tube. $800 \mu \mathrm{l}$ of phenol:chloroform (24:1) was added to the supernatant and mixed well by inverting ten times. This tube was centrifuged at $13000 \mathrm{rpm}$ for $20 \mathrm{~min} .400 \mu \mathrm{l}$ of supernatant was transferred to a new $1.5 \mathrm{ml}$ tube containing $0.6 \mathrm{ml}$ isopropanol and inverted ten times. The tube was centrifuged at maximum speed for $10 \mathrm{~min}$. The pellet was dried at $37^{\circ} \mathrm{C}$ for $30 \mathrm{~min}$ and dissolved in TE buffer. The RNA was eliminated by adding $4 \mu \mathrm{l}$ of RNase $\mathrm{A}(10 \mathrm{mg} / \mathrm{ml})$ at $65^{\circ} \mathrm{C}$ for $30 \mathrm{~min}$. DNA quality and concentration were checked by running a gel and measuring with a Nanodrop spectrophotometer.

Total DNA isolation from tomato materials: Plant roots and hypocotyls were separately ground to fine powder in liquid nitrogen using a mortar and pestle. The fine powder was directly used for DNA extraction by using a DNeasy Plant Mini Kit (Qiagen, Hilden, Germany) with some modification. $1 \mathrm{~g}$ of fine powder was added to a $2 \mathrm{ml}$ tube (two tubes for each repetition). $1000 \mu \mathrm{l}$ of pre-warmed AP1 buffer at $65^{\circ} \mathrm{C}$ and $4 \mu \mathrm{R}$ Rase $\mathrm{A}$ were added to the fine powder and mixed at maximum speed for 20 seconds. The sample was incubated at $65^{\circ} \mathrm{C}$ for $15 \mathrm{~min}$ and inverted 
two to three times during incubation. $250 \mu \mathrm{l}$ of P3 buffer was added to the lysate and mixed well by inverting the tube. The sample was further incubated for $5 \mathrm{~min}$ on ice and centrifuged at the maximum speed for $5 \mathrm{~min}$. The lysate was transferred into a Qiagen Shredder Spin column and centrifuged for $2 \mathrm{~min}$. The flow-through of two tubes of the same sample was collected into a $10 \mathrm{ml}$ falcon. A 1.5 volume of the AW1 buffer was added in that tube and mixed by pipetting. $650 \mu \mathrm{l}$ of the mixture was transferred to a DNeasy Mini Spin column and centrifuged at $8000 \mathrm{rpm}$ for 15 seconds. This step was repeated with the remaining sample. The column was dried by centrifuging for $1 \mathrm{~min}$ and washed twice with $500 \mu \mathrm{l}$ of the AW2 buffer. The column was dried again by centrifuging for $1 \mathrm{~min}$ at $13000 \mathrm{rpm}$ before transferring to a new $1.5 \mathrm{ml}$ tube. The total DNA was eluted by adding $30 \mu \mathrm{l}$ of $\mathrm{AE}$ buffer into the column and collected by centrifuging at maximum speed for $1 \mathrm{~min}$. The concentration of DNA was measured by a Nanodrop spectrophotometer.

RNA isolation: Yeast strains were grown in $10 \mathrm{ml}$ of an activation or part repression media on a rotatory shaker at $30^{\circ} \mathrm{C}$ overnight. The pellet was harvested by centrifugation. $2 \mathrm{ml}$ of spore solution $\left(10^{7} \mathrm{spores} / \mathrm{ml}\right)$ of $V$. dahliae strains was added in $50 \mathrm{ml} \mathrm{SXM} \mathrm{and} \mathrm{grown} \mathrm{on} \mathrm{a} \mathrm{shaker} \mathrm{at} 120 \mathrm{rpm}$ at $25^{\circ} \mathrm{C}$ for three days. Mycelium was harvested using a miracloth membrane. The cell pellet and the mycelium were directly used for RNA isolation. The cell pellet and the mycelium were ground by a Mixer Mill MM 400 (Retsch GmbH, Haan, Germany) with two iron marbles in a $2 \mathrm{ml}$ tube in liquid nitrogen. RNA was extracted by using the RNeasy Plant Mini Kit (Qiagen, Hilden, Germany) according to the manufacturer's protocol.

\subsubsection{PCR amplification}

The Phusion DNA polymerase (Thermo Fisher Scientific GmbH, St. LeonRot, Germany) was used for cloning, checking and confirmation purposes. PCR cycling including an initial denaturation at $98^{\circ} \mathrm{C}$ for 30 seconds -1 min followed by 40 cycles of denaturation at $98^{\circ} \mathrm{C}$ for 10 seconds, annealing at $55^{\circ} \mathrm{C}-68^{\circ} \mathrm{C}$ for 20 seconds, and extension at $72^{\circ} \mathrm{C}$ for 15 seconds $/ \mathrm{kb}$; a final extension at $72^{\circ} \mathrm{C}$ for $5 \mathrm{~min}$ and storage at $8^{\circ} \mathrm{C}$ until used. PCR products were run on a $1 \%$ agarose gel. DNA fragments were isolated and purified with a Qiagen quick gel extraction kit (Qiagen, Hilden, Germany). 


\subsubsection{Southern hybridization}

All deletion and complementation strains were confirmed by Southern hybridization. Fungal genomic DNA was isolated from seven days old mycelium which was grown in liquid PDM on a shaker at $25^{\circ} \mathrm{C}$. The genomic DNA was digested overnight with specific restriction enzymes. Digested DNAs were loaded and processed on $1 \%$ agarose gel for $2 \mathrm{~h}$ at 90 voltage $(\mathrm{V})$. The DNA was denatured before being transferred to an Amersham hybond-N membrane (GE Healthcare, Braunschweig, Germany) by blotting overnight. DNA molecules and the membrane were cross-linked under ultraviolet (UV) light for 3 min for each side. The probes were prepared using the Amersham Alkphos Direct labeling Reagent Kit (GE Healthcare) according to the user's manual and hybridized to the denatured DNA on the membrane at $60^{\circ} \mathrm{C}$ overnight. The probes were detected with the CDP-Star Detection reagent (GE Healthcare) following the user's manual.

\subsubsection{Phenotypical analyses}

\subsubsection{Microsclerotia counting}

The spores of the JR2-wt, the deletion, and complementation strains were dropped on CDM plates containing $3 \%$ cellulose $(\mathrm{w} / \mathrm{v})$ and grown at $25^{\circ} \mathrm{C}$ for seven days. The number of microsclerotia on the surface and in agar was counted under a binocular microscope (Leica M165 FC). The mean value was calculated from three independent replicates.

\subsubsection{Conidia examination}

The formation of spores in liquid medium and conidiospore clusters on agar plates was examined. The same number of spores of indicated strains was grown in $50 \mathrm{ml} \mathrm{SXM}$ on a shaker at $120 \mathrm{rpm}$ to test spore formation. The number of spores in culture media was counted at seven dpi using a Thoma counting chamber (Paul Marienfeld GmbH\&Co.KG, Lauda-Königshofen, Germany). The experiment was performed in triplicate. The formation of conidiospore clusters was observed on CDM plates. The same number of spores of indicated strains was dropped on CDM plates and grown in the dark for three days. The formation of conidiospore cluster was observed with a binocular microscope. 


\subsubsection{Hyphal branching test}

The same number of spores of the indicated strains was dropped on agar plates and grown in the dark at $25^{\circ} \mathrm{C}$ for two days. The hyphal branching was observed under the microscope, and the number of branches was further quantified by multiple micro channels (Stanley et al., 2014). A piece of agar medium containing fungal mycelium was cut and placed next to the device opening. The growth direction of the hyphal tips was orientated toward the micro channels. In order to count the number of hyphal branches, the fungi were grown in the dark for two days. The number of hyphal branches was counted in $750 \mu \mathrm{m}$ length of hyphae from the tip. The experiments were performed in triplicate with 20 hyphae per each repetition.

\subsubsection{Localisation study}

Fragments harboring $1.2 \mathrm{~kb}$ upstream and the ORF without stop codon of SOM1 and VTA3 were fused to the N-terminal of a GFP gene in the pGreen2 (Tran et al., 2014) background between Spel and Kpnl to generate pME4551 and pME4556 plasmids. These plasmids were transformed into the deletion strain VGB0001 or VGB0009 to produce VGB0084, VGB0085, VGB0284, and VGB0285 via the A. tumefaciens (Timpner et al., 2013). The transformants were selected on PDA plates containing hygromycin $(50 \mu \mathrm{g} / \mathrm{ml})$ and cefotaxime $(100 \mu \mathrm{g} / \mathrm{ml})$ and screened under a fluorescent microscope with the green filter. The positive clones were further examined by Southern hybridisation. Localisation was examined by fluorescence microscopy. Fungal nuclei were stained with 4',6-diamidino-2phenylindole (DAPI).

\subsubsection{Oxidative stress test}

One volume of $100 \mu \mathrm{l}$ of spore suspension $\left(10^{7}\right.$ spores/ $\mathrm{ml}$ ) of JR2-wt, the deletion or complementation strains was spread on PDA plates containing cefotaxime $(100 \mu \mathrm{g} / \mathrm{ml})$ with glass beads. A paper disc with a diameter of $6-8 \mathrm{~mm}$ containing $10 \mu \mathrm{l}$ of $10 \% \mathrm{H}_{2} \mathrm{O}_{2}$ or menadione $(100 \mathrm{mg} / \mathrm{ml})$ was placed in the center of each plate. Plates were incubated in the dark at $25^{\circ} \mathrm{C}$ for four days to show growth inhibition effects. These experiments were performed in triplicate. 


\subsubsection{Adhesion examination}

Yeast adhesion test

The GAL1 promoter is activated in SC-Uracil containing $2 \%$ galactose and the MET25 promoter is part repressed in SC-Uracil medium containing $2 \%$ glucose. Yeast strains were grown on induction or part repression medium for three days at $30^{\circ} \mathrm{C}$. The plates were washed under water stream until negative strain was washed off. The plates were photographed before and after washing.

For flocculation in liquid medium, S. cerevisiae strains were cultivated in 10 $\mathrm{ml}$ induction or part repression media on a rotator for one day at $30^{\circ} \mathrm{C}$. The flocculation part and non-flocculation part were separately dried and measured. The experiment was performed in triplicate.

Verticillium adhesion test

Adhesion of $V$. dahliae on abiotic surfaces was examined with two different surfaces polystyrene and hydrophobic GelBond film. The JR2-wt, SOM1 deletion, and SOM1 complementation strains were grown on a polystyrene plate containing SXM for seven days at $25^{\circ} \mathrm{C}$. The wells were washed with a water stream. The polystyrene plate was photographed before and after washing. On hydrophobic GelBond film surfaces, the same number of spores of indicated strains in 1/10 liquid minimal medium (MM) were placed on the surface and incubated at $25^{\circ} \mathrm{C}$ in the dark for $24 \mathrm{~h}$. The GelBond film surfaces were washed with water and further processed under a microscope. All experiments were performed in triplicate.

\subsubsection{Plant infection test}

\subsubsection{Tomato infection study}

The tomato infection assay was modified from (Tran et al., 2014). Ten-day-old tomato seedlings "MoneyMaker" (Bruno, Nebelung GmbH, Everswinkel, Germany) were infected with $10 \mathrm{ml}$ spore solution $\left(2 \times 10^{6}\right.$ spores $\left./ \mathrm{ml}\right)$ of the wild-type, deletion, and complementation strains or non-infected with tap water for $30 \mathrm{~min}$ by root dipping. Then, they were transferred into pots containing a mixture of sand and soil $(1: 1)$. Tomato plants were kept in the dark for $24 \mathrm{~h}$ to reduce stress effects. The infected plants were cultivated in a climate chamber with $16 \mathrm{~h}$ light and $8 \mathrm{~h}$ dark 
at $22-25^{\circ} \mathrm{C}$. The soil humidity was controlled at a minimum level to increase pathogenic symptoms. The plant heights were measured weekly until 35 days post infection (dpi). The fungus re-isolation from infected plants was modified from (Tran et al., 2014). Hypocotyls of infected plants were sterilized in the solution containing $6 \%$ hypochlorite and $0.05 \%$ Tween 80 for $7 \mathrm{~min}$, ethanol $70 \%$ for $5 \mathrm{~min}$ and washed twice with distilled water for $5 \mathrm{~min}$. The sterilized hypocotyl was placed on PDA plates containing chloramphenicol $(100 \mu \mathrm{g} / \mathrm{ml})$ and cefotaxime $(100 \mu \mathrm{g} / \mathrm{ml})$ and cultivated at $25^{\circ} \mathrm{C}$ for seven days. The total DNA was extracted from roots and hypocotyls. The fungal DNA in total DNA was quantified by real-time PCR with OLG primers (Eynck et al., 2007; Timpner et al., 2013). The experiments were repeated twice with 16 plants per each treatment.

\subsubsection{Arabidopsis root infection test}

Root infection test was modified from (Tran et al., 2014). The Arabidopsis thaliana seeds were sterilized in a solution containing $70 \% \mathrm{EtOH}$ and $0.1 \%$ Tween 80 for $5 \mathrm{~min}$ and in $\mathrm{EtOH} 99 \%$ for $5 \mathrm{~min}$ and dried at $37^{\circ} \mathrm{C}$ for $1 \mathrm{~h}$. The sterilized seeds were grown on Murashige and Skoog plates (Duchefa, Haarlem, Netherlands), which include 1\% sucrose, 0.05\% MES (Carl Roth, Karlsruhe, Germany), and $1.5 \%$ plant agar (Duchefa, Haarlem, Netherlands). The $\mathrm{pH}$ was adjusted to 5.7. Arabidopsis plants were cultivated in a climate chamber under $16 \mathrm{~h}$ light and $8 \mathrm{~h}$ dark at $22-25^{\circ} \mathrm{C}$. Three week old plants were transferred to $1 \%$ agarose plates and incubated for $24 \mathrm{~h}$. The plant roots were dipped in the spore solution $\left(10^{5} \mathrm{spores} / \mathrm{ml}\right)$ of the wild-type or the deletion strain expressing GFP for $35 \mathrm{~min}$. Infected plants were put back to agarose plates and co-inoculated in a climate chamber. The root area was covered with aluminum foil for shading. Before microscopy, plant roots were stained with a solution containing propidium iodide $0.0025 \%$ and silvet $0.0005 \%$. Confocal laser scanning microscope (Leica SP5) pictures were taken at different time points.

\subsubsection{Scan electron microscopy}

After a fixation step (2.5\% glutaraldehyde, $2 \%$ paraformaldehyde, $1 \mathrm{mM} \mathrm{CaCl}_{2}$, $1 \mathrm{mM} \mathrm{MgCl}_{2}$, Tween 20, $50 \mathrm{mM} \mathrm{NaN}_{3}$ in $100 \mathrm{mM}$ HEPES pH 7.5), samples were treated with amino acid-sucrose-solution (2\% arginine, $2 \%$ glycine, $2 \%$ glutamate, $2 \%$ sucrose) for one hour, guanidine-tannin-solution ( $2 \%$ guanidine, $2 \%$ tannin) for $30 \mathrm{~min}$, and $1 \%$ osmium tetroxide in cacodylate buffer $(\mathrm{pH} \mathrm{7}$; supplemented with $1 \mathrm{mM} \mathrm{CaCl}_{2}, 1 \mathrm{mM} \mathrm{MgCl}_{2}, 50 \mathrm{mM} \mathrm{NaNO}_{3}$ ) for $30 \mathrm{~min}$ at room temperature with 
washing steps in between. The samples were dehydrated in a graded series of aqueous ethanol solutions (10-100\%) and then critical point-dried. Finally, samples were mounted on aluminum stubs, sputtered with gold/palladium and examined with a scanning electron microscope EVO LS10 (Carl Zeiss Microscopy GmbH, Oberkochen, Germany).

\subsubsection{Protein methods}

\subsubsection{Protein isolation}

The cell lysate proteins were isolated from three days old mycelium which was grown in $100 \mathrm{ml}$ liquid $\mathrm{MM}$ on a shaker at $120 \mathrm{rpm}$ at $25^{\circ} \mathrm{C}$. The mycelium was ground with liquid nitrogen and proteins were extracted with extraction buffer $B$ (Schinke et al., 2016).

\subsubsection{Proteomic analysis}

The same amount of cell lysate proteins $(40 \mu \mathrm{g})$ of JR2-wt, SOM1 deletion, and SOM1 complementation strains were loaded in a $12 \%$ SDS-PAGE and run at $150 \mathrm{~V}$ for $30 \mathrm{~min}$. Proteins in gels were stained with coomassie blue following the staining method (Neuhoff et al., 1985; Neuhoff et al., 1988). Each lane was cut into eight pieces. They were individually digested with trypsin in gels and peptides were treated with C18 stage tips as described by (Rappsilber et al., 2007). Peptide from each piece was independently analysed by mass spectrometry and examined by the MaxQuant program. The list of proteins was filtered by the following workflow: (1) remove all contaminant including "only identified by site", "reverse", and "potential contaminant"; (2) transfer LFQ intensity to Log2 (LFQ intensity) and replace missing values by 1 ; (3) create group 1 base on Log2 (LFQ intensity) containing +SOM1 (JR2-wt and SOM1 complementation strains) and -SOM1 (SOM1 deletion strain) groups; (4) run two-sample tests between + SOM1 and -SOM1 groups. Test type: student's t-test, use for truncation: permutation base FDR, FDA = 0.05; (5) filter to remove all non-significant different; (6) filter and keep all protein with three times Log2 $(\mathrm{LFQ}+S O M 1)>20$; (7) filter and keep all protein with t-test different $>10$. 


\subsubsection{Western hybridization}

Extracted proteins $(40 \mu \mathrm{g})$ in the SDS loading dye were added to a $12 \%$ SDS-PAGE after heating at $95^{\circ} \mathrm{C}$ for $5 \mathrm{~min}$, run for one hour at $150 \mathrm{~V}$ and transferred to a nitrocellulose membrane (GE Healthcare Europe $\mathrm{GmbH}$, Freiburg, Germany). The GFP signal was detected by a mouse a-GFP antibody (Santa Cruz Biotechnology, Cat no: Sc-9996) (1:1000) and HRP conjugated goat mouse secondary antibody (Jackson Immunoresearch, Cat no: 115-035-003) (1:2000). Antibodies were diluted in TBST with $5 \%(\mathrm{w} / \mathrm{v})$ non-fat dry milk.

\subsubsection{GFP trap assay}

Proteins of the fungal strains VGB0084, VGB0085, VGB0281, and VGB0282 were isolated as described above. Total protein extract was processed with $25 \mu$ l GFP-Trap beads (Chromo Tek GmbH, Planegg-Martinsried, Germany) according to user's manual. Eluted proteins were applied to a $12 \%$ SDS-PAGE and run for $13 \mathrm{~min}$ to check the quality of purified proteins. Gel pieces were isolated and digested with trypsin in gels according to Shevchenko's protocol (Shevchenko et al., 1996). Peptides digested by trypsin were treated with C18 StageTips (Rappsilber et al., 2007) before performing Mass spectrometry analysis (Tran et al., 2014). Orbitrap raw files were analysed with the MaxQuant program (version 1.4.1.2) and protein list was filtered by Perseus (version 1.5.5.3).

The list of protein was sequentially filtered by the following workflow: (1) remove all contaminant including "only identified by site", "reverse", and "potential contaminant"; (2) transfer LFQ intensity to Log2 (LFQ intensity) and replace missing values by 1 ; (3) create group 1 containing of free GFP strain and group 2 comprising log2 (LFQ intensity) of SOM1:GFP strains; (4) remove rows if the group 1 is higher than 1; and (5) remove rows if 3 times of group 2 smaller than 10 . The free GFP in VdJR2 wild-type was used as a negative control. The experiments were performed with three biological and three technical replicates in two independent strains.

\subsubsection{Gene expression quantification}

The gene expression was quantified with qPCR MasterMix (5 PRIMER, Hamburg, Germany) using the CFX Connect Real-Time PCR system (Bio-Rad Laboratories $\mathrm{GmbH}$, München, Germany). Total RNAs were extracted from mycelium of $V$. dahliae which is grown in liquid SXM for three days or cells of $S$. cerevisiae 
incubated overnight using the RNeasy Plant Mini Kit (Qiagen). The cDNA was synthesized using $0.8 \mu \mathrm{g}$ of RNA with the Quantitech ${ }^{\mathrm{R}}$ Reverse Transcription Kit (Qiagen, Hilden, Germany). The relative expression of the genes was calculated using the $\triangle \triangle \mathrm{Cq}$ method (Haimes \& Kelley, 2014). GAPDH, PP2A1, and PP2A2 were used as housekeeping genes for Verticillium or TAF10 and ALG9 for S. cerevisiae. Real-time PCR reactions contained $4 \mu$ of the 2.5x MasterMix included SYBR, $5 \mathrm{pmol}$ for forward and reversed primers, $100 \mathrm{ng}$ cDNA and water in a final volume of $10 \mu \mathrm{l}$. All primers used for quantification are listed in Table 1. The experiment was performed with two independent deletion and complementation strains in triplicates. 


\section{$3 \quad$ Results}

\subsection{The transcription factors SOM1 and VTA3 can reprogram non-adhesive yeast strain}

Vta3 is an ortholog of transcriptional repressor Rfx1, whereas Som1 is a potential homolog of Flo8 in S. cerevisiae (Tran et al., 2014). In this study, the effect of Som1 and Vta3 in adhesion was examined in a non-adhesive yeast strain. The mechanism of Som1 and Vta3 in adhesion in yeast model were investigated. The genomic DNA and amino acid sequences of transcription factors SOM1 and VTA3 were analysed by bioinformatics programs and encoded protein domains were compared to the homologs in other fungi. The expression of relevant genes for adhesion in yeast was tested by real-time PCR.

\subsubsection{SOM1 and VTA3 genes encode proteins comprising a LisH or a wing helix-turn-helix DNA binding domain}

The SOM1 and VTA3 sequences were analysed with NCBI blast, Pfam, 9aaTAD, and SeqNLS to find homologs in other fungi and to predict the domains and the nuclear localisation signal (Figure 15, 16). V. dahliae Som1 (XP_009650808) shows similarity to S. cerevisiae Flo8 (23\%), AfSomA from A. fumigatus (40\%), and MoSom 1 from M. oryzae (51\%).

Alignments of the CDNA and the gDNA sequence of SOM1 shows that the gene structure consists of six exons and five introns resulting in an open reading frame of 822 codons for a protein with a predicted molecular weight of $86.6 \mathrm{kDa}$ (Figure 15). The results of the bioinformatic analysis indicated that the Som1 sequence harbors a LisH domain at its $\mathrm{N}$-terminus, a SnAPC domain at the C-terminus, an SSDP domain in the middle and two putative nuclear localisation signals (NLS). Orthologs to the LisH domain were found in Aspergillus fumigatus, Aspergillus nidulans, and Magnaporthe oryzae with 50\%, 54\%, and $60 \%$ amino acid similarity, respectively. Moreover, it also displayed high comparability to orthologs in pathogens Colletotrichum gloeosporioides, Diaporthe ampelina, Ustilaginoidea virens, Fusarium oxysporum, and Metarhizium rileyi: $71 \%, 73 \%, 79 \%, 79 \%$, and $74 \%$ amino acid similarity, respectively (Figure 15 ). 


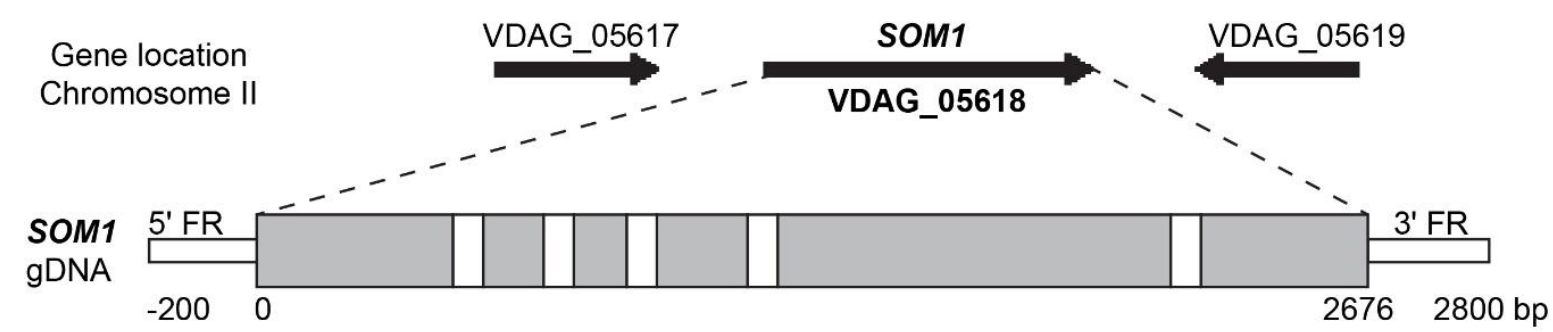

SOM1 mRNA
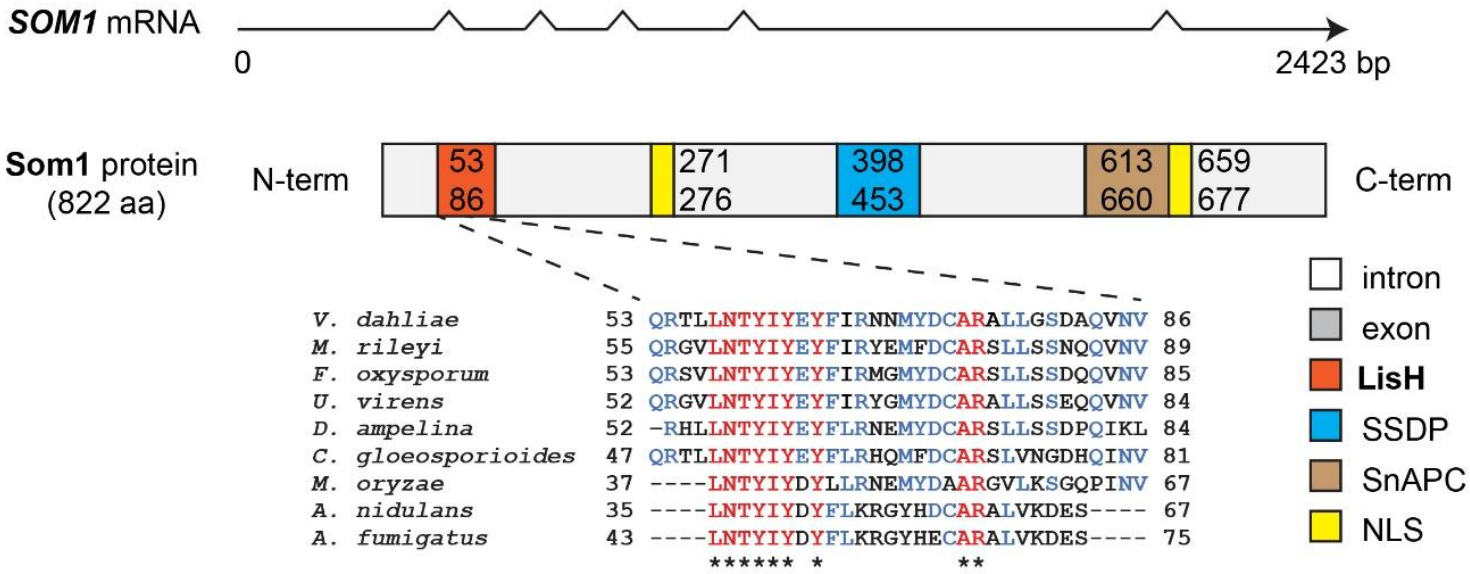

Figure 15. Gene locus and structure of SOM1. Genomic DNAs were compared with cDNA using MultAlin. Introns and exons were marked. The nuclear localisation signals (NLS) predicted by the SeqNLS software are displayed. The protein domains were predicted by three different programs: Pfam, NCBI blast, and 9aaTAD. Sequence alignments of the LisH domain of Som1 and related proteins from other organisms including Metarhizium rileyi (OAA39058), Fusarium oxysporum (XP_018248456), Ustilaginoidea virens (KDB12219), Diaporthe ampelina (KKY33496), Colletotrichum gloeosporioides ((XP_007286727), Magnaporthe oryzae (XP_003710835), Aspergillus nidulans (XP_682356), and Aspergillus fumigatus (XP_746706) are shown. Asterisks: identical residues; Red: high (90\%), blue: low (50\%) consensus values; FR: flanking region; N-term: N-terminus; C-term: C-terminus.

The VTA3 gene contains two exons and one intron; the ORF encodes a 795 aa protein with predicted molecular weight of $87.4 \mathrm{kDa}$ (Figure 16). The Vta3 protein sequence was analysed by bioinformatics programs and the data showed that it contains a wing helix-turn-helix DNA binding domain (WDBD), three different nine amino acid transactivator domains (9aaTAD), and one putative NLS (Figure 16). The WDBD domain showed little similarities to the ortholog in Candida albicans (48\%), but similarities to the orthologs in A. fumigatus, A. nidulans, Penicillium chrysogenum, and Acremonium chrysogenum: 64\%, 64\%, 64\% and $71 \%$, respectively. It also showed high similarity to the orthologs in F. graminearum, U. virens, and Metarhizium brunneum: 95\%, 99\%, and 99\%, respectively (Figure 16). 


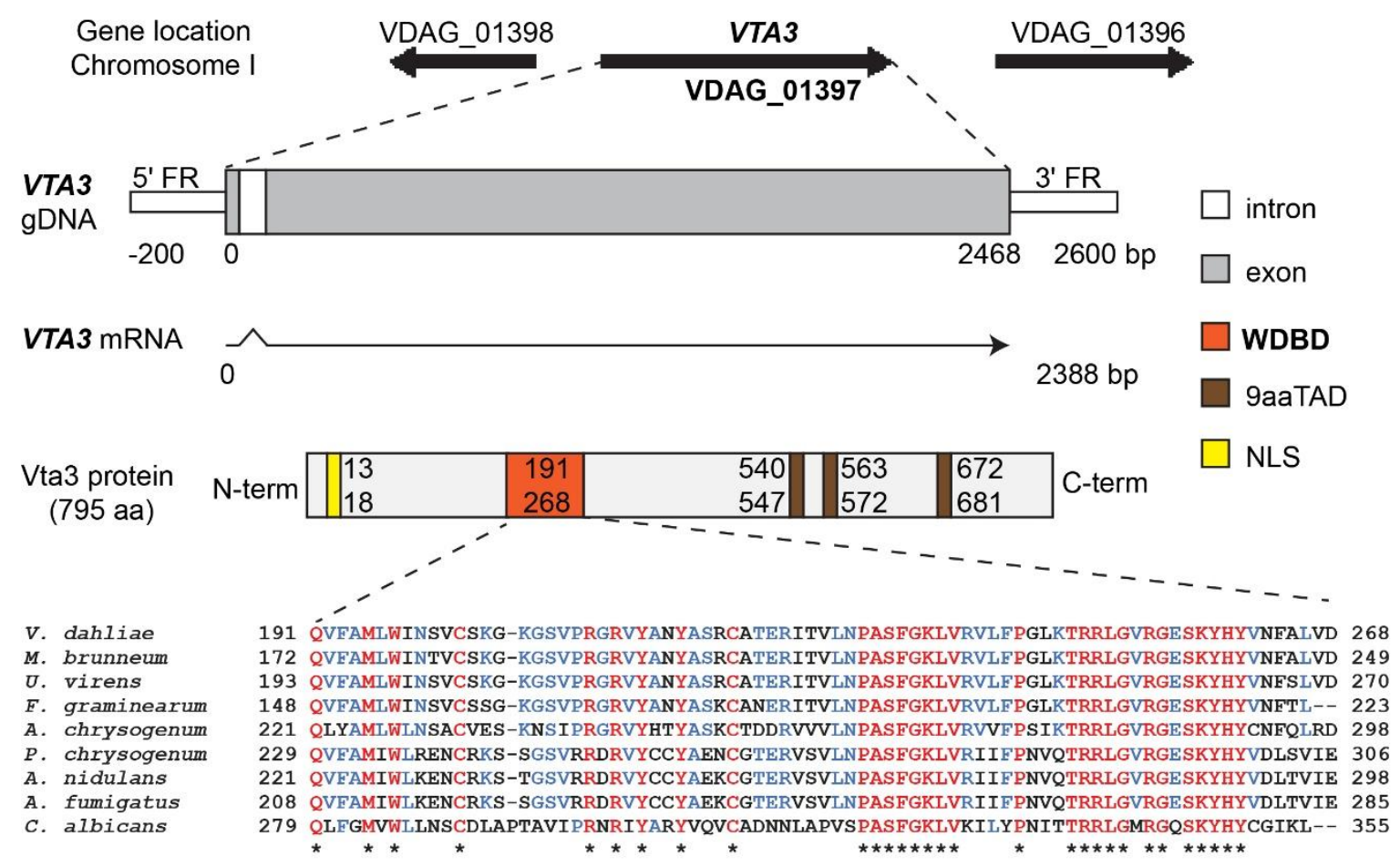

Figure 16. Gene locus and structure of VTA3. Introns and exons are marked. The predicted NLS and putative protein domains are indicated. Multiple alignments of (WDBD) of Vta3 and the corresponding proteins from other fungi include Metarhizium brunneum (XP_014546736), Ustilaginoidea virens (GAO17021), Fusarium graminearum (XP_011327190), Acremonium chrysogenum (CAB85587), Penicillium chrysogenum (CAB85619), Aspergillus nidulans (XP_659900), Aspergillus fumigatus (XP_754020), and Candida albicans (XP_713492). Asterisks: identical residues; Red: high (90\%), blue: low $(50 \%)$ consensus values; FR: flanking region; N-term: N-terminus; C-term: C-terminus.

\subsubsection{Som1 and Vta3 are nuclear proteins}

Both, SOM1 and VTA3, sequences contain a putative NLS, therefore, the localisation of these proteins in the cell was studied. Consequently, SOM1 and VTA3 genes under the control of their native promoter were fused to the GFP gene in pGreen2 (Tran et al., 2014) to examine the subcellular localisation of Som1 and Vta3. Each construct was transferred into the corresponding deletion strain of SOM1 and VTA3, respectively. The GFP signal of the transformation strains was observed by fluorescence microscopy after $24 \mathrm{~h}$ growth in SXM. Fungal nuclei were stained by 4',6-diamidin-2-phenylindol (DAPI).

The GFP signal was strongly detected in nuclei of the strains expressing SOM1::GFP or VTA3::GFP as shown in Figure 17. The GFP signal co-localised with the DAPI stained nuclei (Figure 17). Contrastingly, the GFP signal was identified throughout the whole hyphae in control cells expressing only free GFP (JR2-GFP) and it was not found in the wild-type strain (JR2-wt). The results indicate that both, Som1 and Vta3, are nuclear proteins. 
$\mathrm{DIC}$
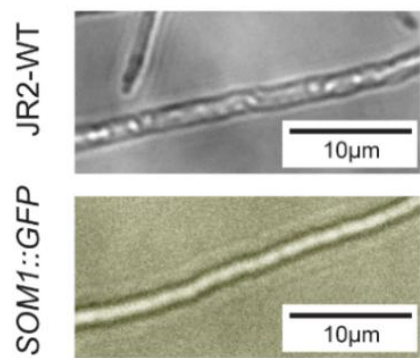

号
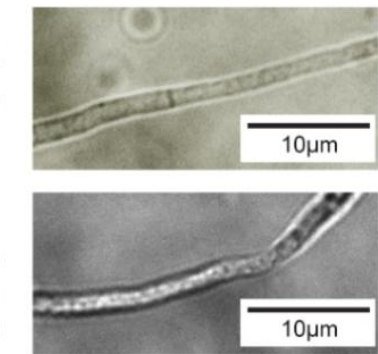

GFP
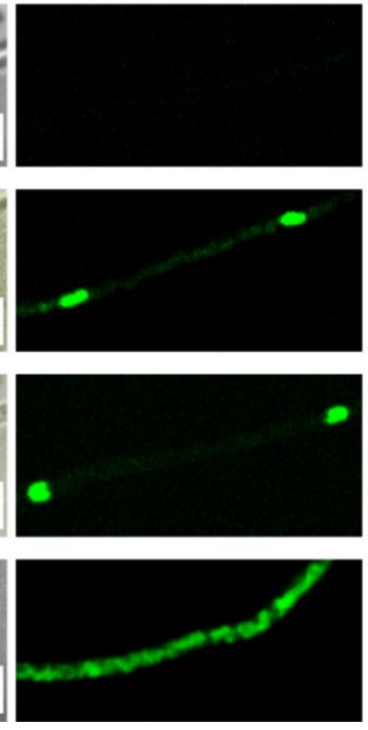

DAPI
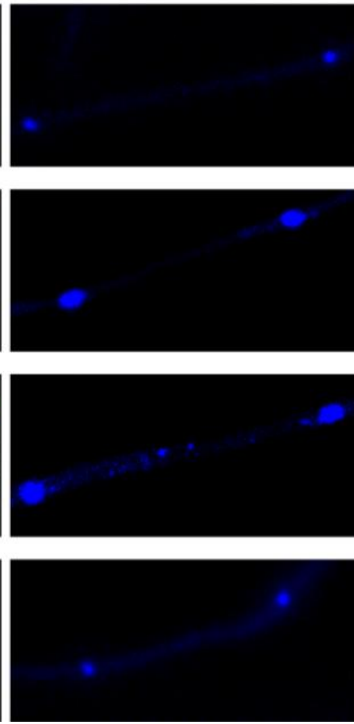

merged
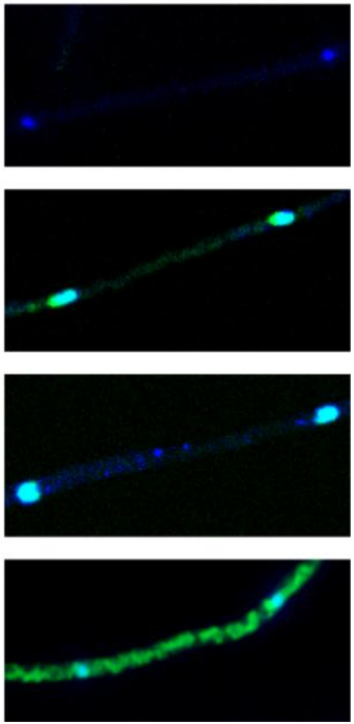

Figure 17. Som 1 and Vta3 are nuclear proteins. The SOM1 and VTA3 genes under the control of the native promoter were fused to GFP separated by the GGSGG linker and ectopically transformed into the respective strains. Transformants were screened on selective medium containing hygromycin $50 \mu \mathrm{g} / \mathrm{ml}$ and examined by fluorescence microscopy. The positive clones with a strong GFP signal were grown in liquid SXM for $24 \mathrm{~h}$. The localisation of proteins was observed by green fluorescence. Fungal nuclei were stained with 4',6-diamidin-2-phenylindol (DAPI). The strain names and filter are shown. Scale bars are represented.

\subsubsection{Som1 and Vta3 can rescue FLO8-defective S. cerevisiae strains}

To examine whether SOM1 and VTA3 can complement the FLO8 deletion strain, adhesion experiments were performed on agar plates and in liquid medium. Strains harbouring the respective ORFs of SOM1 and VTA3 were under the control of either the GAL1 promoter or MET25, and subsequently transformed to a yeast FLO8-deletion strain. The low expression of SOM1 was achieved in SC-uracil medium where the MET25 promoter is partially repressed whereas overexpression of SOM1 was attained in SC-uracil + galactose or SC-uracil-methionine where the GAL1 and the MET25 promoters are induced. The adherence test to agar plates shown in Figure 18 clearly demonstrates that the yeast strain expressing SOM1 under the control of either the GAL1 or the MET25 promoter is unable to bind to agar plates. It was easily washed off as seen also for the negative control ((-) control). However, yeast strains with low expression of SOM1 under the control of the MET25 promoter in SC-uracil medium or high expression of VTA3 under the control of the GAL1 promoter can reprogram non-adhesive FLO8-defective yeasts to adhesion. They still bind on agar plates after washing (Figure 18). 
(a)
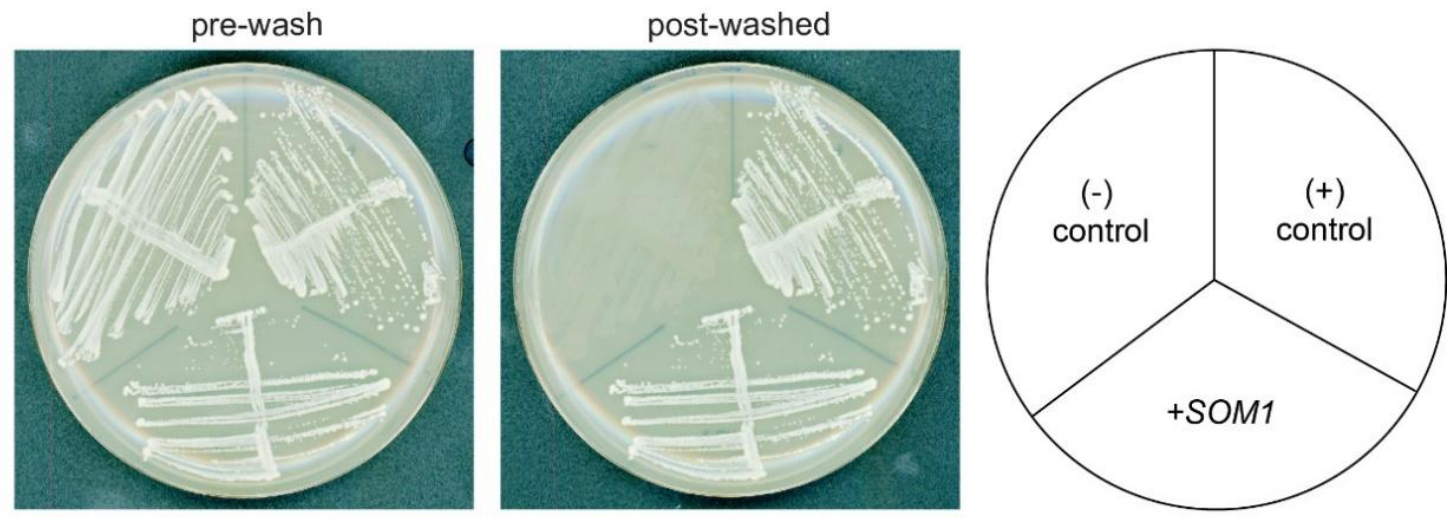

(b)
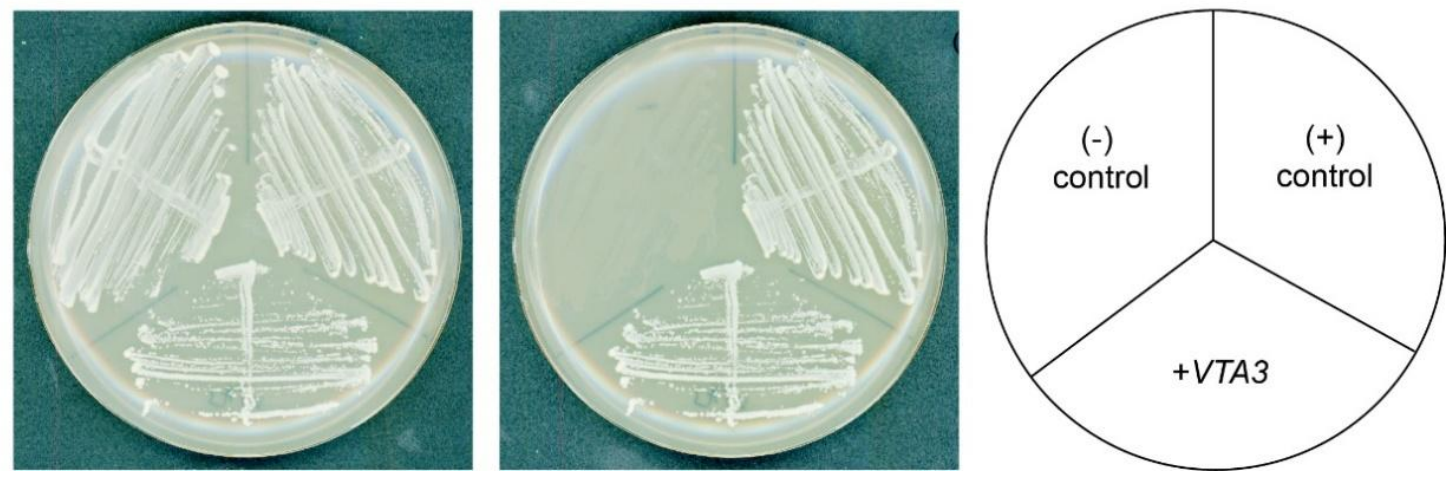

Figure 18. SOM1 and VTA3 can reprogram non-adhesive FLO8-deficient S. cerevisiae strains to adhesion on agar plates. Haploid $S$. cerevisiae strain BY4741 expressing SOM1 or BY4742 expressing VTA3. The S. cerevisiae FLO8 mutant strain with empty vector served as negative (-) control and the strain with a vector harboring FLO8 was used as a positive (+) control. (a) Adhesion of the BY4741 strain with low expression of V. dahliae SOM1 in SC-Ura+glucose medium. (b) Adherence of the BY4742 strain with high expression of $V$. dahliae VTA3 in the SC-Ura + galactose medium were examined. The adhesion test was performed after two days of growth on agar plates. The plates were washed with water in the sink until the negative control was washed off. All experiments were performed in triplicates.

The flocculation experiment in liquid medium indicates that the negative control and the strains overexpressing SOM1 are unable to flocculate, whereas the positive control and strains with low expression of SOM1 or high expression of VTA3 can flocculate well (Figure 19b). The ratio of flocculation of low expression of SOM1 and high expression of VTA3 is 0.42 and 0.35 , respectively, whereas that of positive and negative controls is 0.55 and 0.00 , respectively (Figure 19a). The expression of genes related to adhesion was further examined. 
(a)

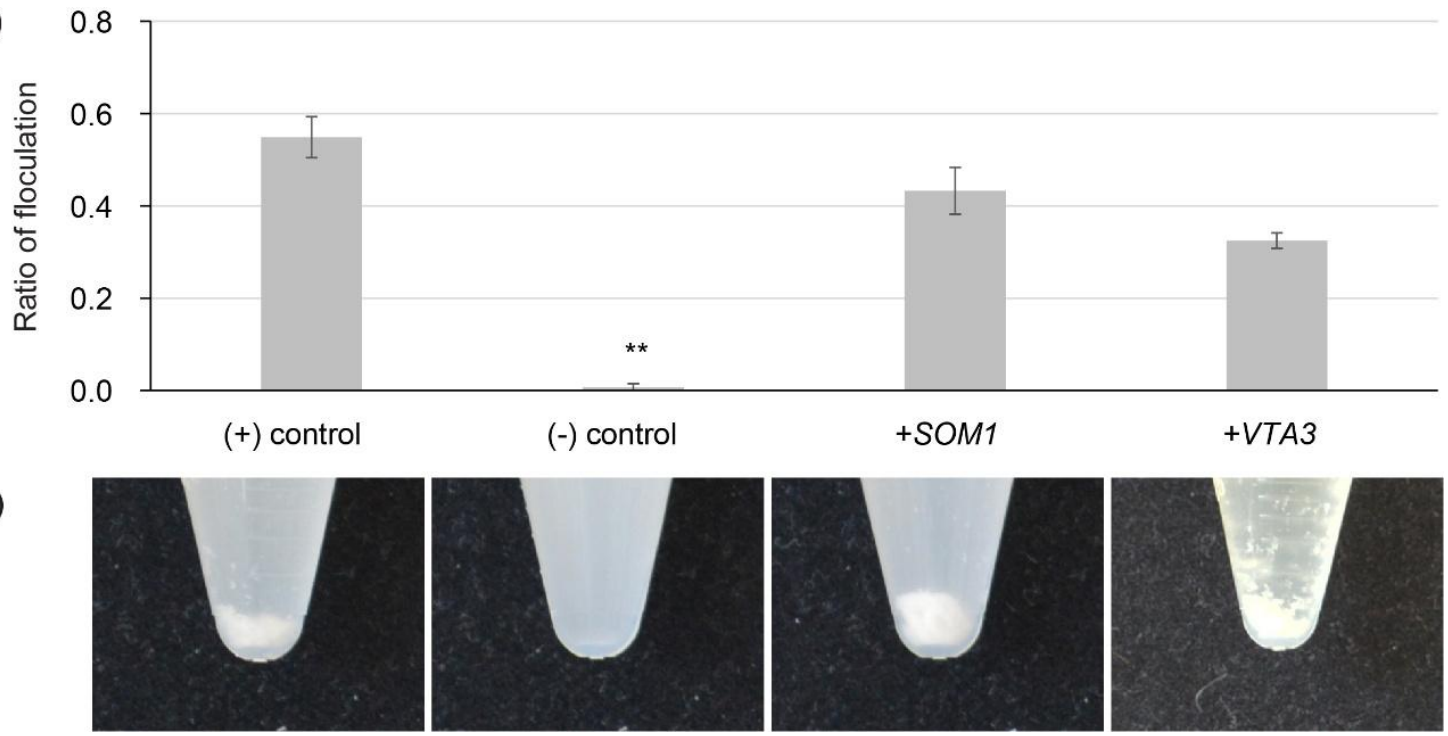

Figure 19. Som1 and Vta3 can activate flocculation of FLO8-defective S. cerevisiae in liquid medium. The BY4741 strain with low expression of $V$. dahliae SOM1 in SC-Ura + glucose medium and the BY4742 strain with high expression of $V$. dahliae VTA3 in the SCUra + galactose medium were examined. The $S$. cerevisiae FLO8 mutant strain with empty vector served as negative (-) control and the strain with a vector harboring FLO8 was used as a positive (+) control. Flocculation tests were carried out by growing indicated strains in $10 \mathrm{ml}$ medium on a rotator for one day at $30^{\circ} \mathrm{C}$. (a) Diagram of ratio of flocculation. The flocculation part and non-flocculation were separately dried and measured. Ratio of flocculation was calculated based on dry weight of flocculation part in total dry mass. (b) Flocculation in liquid medium. Pictures of the cultures with precipitated cells are represented. All experiments were performed in triplicates. Mean values and standard deviation are shown. The asterisk $\left(^{* *}\right)$ indicates a significant decrease when compared to a positive control (Student's t-test, $p<0.01$ ).

\subsubsection{Low expression of SOM1 can activate flocculation genes}

Expression of relevant genes for adhesion in yeast strains expressing SOM1 was further analysed by real-time PCR. The results show that FLO1 and FLO11 are not activated neither in the strain overexpressing SOM1 nor in the negative control, whereas their expression is significantly increased either by the expression of FLO8 or by low expression of SOM1 (Figure 20). Additionally, the overexpression of SOM1 in S. cerevisiae causes the repressive expression of STE12. Expression of STE12 is significantly decreased in this case (Figure 20). Taken together, low expression of SOM1 can activate FLO1 and FLO11, whereas high expression does not. 


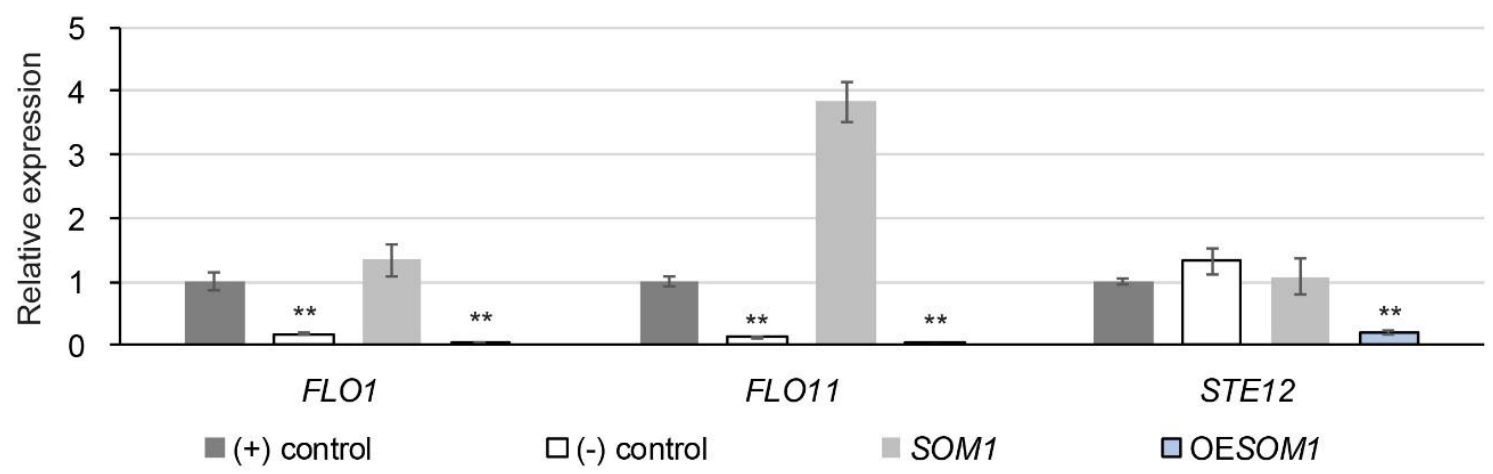

Figure 20. Som1 promotes the expression of FLO1 and FLO11 in FLO8-deficient S. cerevisiae. The RNAs of indicated strains were isolated from the cells which were cultivated in liquid medium for $24 \mathrm{~h}$. The relative expression of genes related to adhesion was normalized to expression of the TAF10 reference gene and the positive control. All experiments were performed in triplicates. Mean values and standard deviation are shown. The asterisk $\left(^{* *}\right)$ indicates a significant decrease when compared to a positive control (Student's t-test, $p<0.01$ ).

\subsubsection{Activation of VTA3 can stimulate expression of flocculation genes}

As expression of VTA3 under the control of the GAL1 promoter can rescue adhesion of non-adhesive yeasts (Figure 18), the effect of Vta3 on the expression of relevant genes for adherence was also examined by RT-PCR (Figure 21). The expression of VTA3 under the control of the GAL1 promoter stimulates the expression of FLO1 and FLO11. Their expression is significantly increased compared to the negative control (Figure 21). Interestingly, the expression of other adhesion-related genes such as STE12, TEC1, PHD1, FLO10, and TPK2 does not change (data not shown), whereas the expression of gene for the repressor Sfl1 is significantly reduced (Figure 21). This means, Vta3 represses the expression of SFL1 to de-repress the expression of flocculation genes including FLO1 and FLO11 and the yeast can adhere on a surface. 


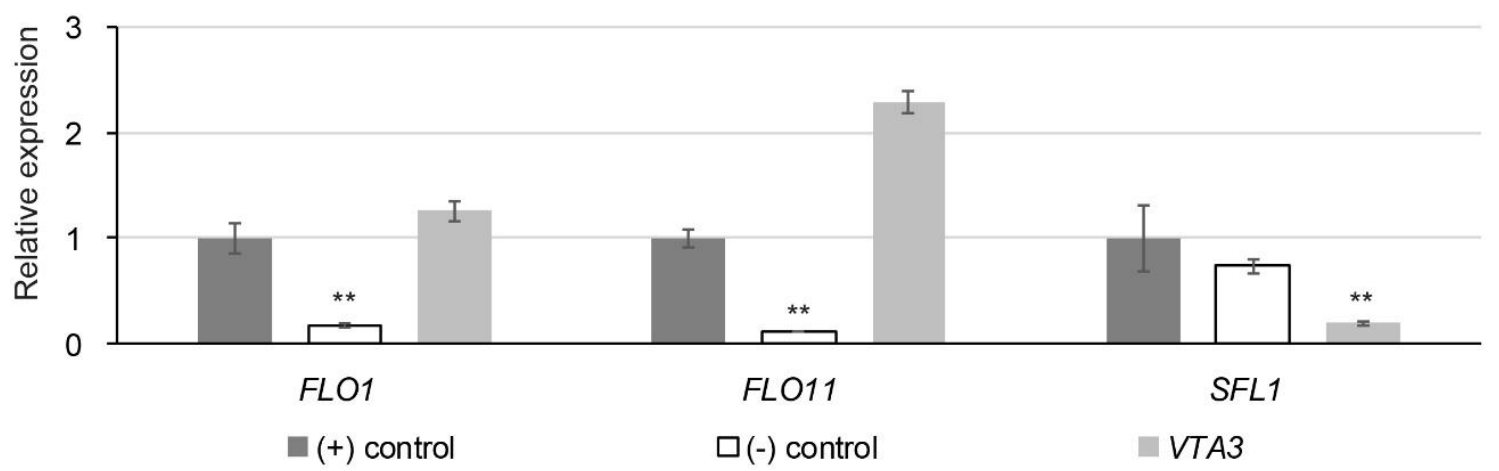

Figure 21. Vta3 stimulates the expression of FLO1 and FLO11 in FLO8-defective S. cerevisiae. The RNAs of indicated strains were isolated from the cells which were cultivated in liquid medium for $24 \mathrm{~h}$. The relative expression of genes related to adhesion was normalized to the expression of the TAF10 reference gene and the positive control. All experiments were performed in triplicates. Mean values and standard deviation are shown. The asterisk $\left({ }^{* *}\right)$ indicates a significant decrease when compared to a positive control (Student's t-test, $p<0.01$ ).

\subsection{Transcription factors SOM1 and VTA3 are required for morphology and virulence in $V$. dahliae}

The functions of Som 1 and Vta3 in V. dahliae were examined by using genetics, cell biology, proteomics, and plant infection experiments. The single deletion of SOM1 and $V T A 3$, and ectopic complementation strains were generated. The phenotypes of deletion strain were compared to that of the wild-type and complementation to address the function of genes.

\subsubsection{Deletion and complementation of SOM1 and VTA3 in V. dahliae}

Deletion and complementation strains of SOM1 and VTA3 were confirmed by Southern hybridization before functional analysis. The deletion of SOM1 was confirmed with two different restriction enzymes, Bsu15l and Eco81l. The wild-type genomic DNA was used as a control. The expected bands of $5.7 \mathrm{~kb}$ and $1.9 \mathrm{~kb}$ were observed in the deletion strain, and 3.0 and $3.3 \mathrm{~kb}$ for the wild-type, respectively (Figure 22). The ectopic complementation of SOM1 was confirmed with Xbal/Pvul enzymes, using the genomic DNA of the SOM1 deletion strain as a control. The expected bands for the complementation $(1.7 \mathrm{~kb}$ and $4.9 \mathrm{~kb})$ and the control strain $1.7 \mathrm{~kb}$ were observed as seen in Figure 22. 


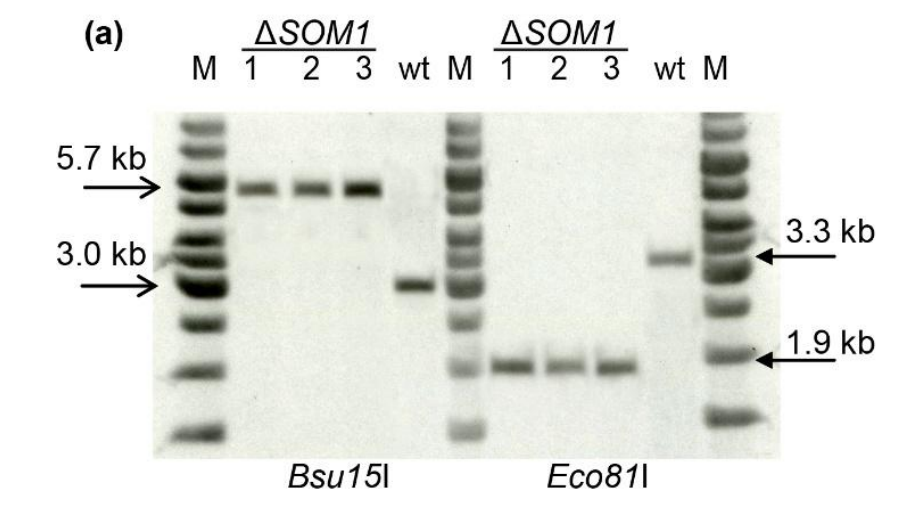

(b)
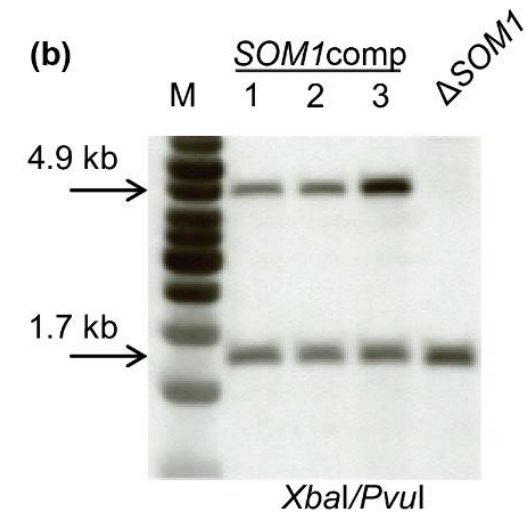

Figure 22. Confirmation of deletion and complementation strains of $V$. dahliae SOM1. (a) Confirmation of deletion strains by Southern hybridization with two different restriction enzymes. The probe is the 3' flanking region. Genomic DNA of the wild-type was used as a control. (b) Confirmation of complementation strains by Southern hybridization with Xbal and Pvul. Genomic DNA of the SOM1 deletion strain was used as a control. The probe is the terminator of SOM1 of $0.8 \mathrm{~kb}$. Sizes of expected fragments are indicated. Restriction enzymes and predicted sizes are shown.

Next, deletion and complementation strains of VTA3 were constructed and confirmed by Southern hybridization (Figure 23). The VTA3 deletion strain was confirmed by Southern hybridization with two different enzymes BspEl and Xhol. Genomic DNA of the wild-type was used as a control. The expected bands of $1.8 \mathrm{~kb}$ and $4.0 \mathrm{~kb}$ were observed in the genome of the VTA3 deletion strain, and $5 \mathrm{~kb}$ and $2.3 \mathrm{~kb}$ bands for the genome of the wild-type. The VTA3 complementation strain was confirmed with the restriction enzyme Avall, using the genomic DNA of the VTA3 deletion strain as a control. The expected bands for the complementation are $1.8 \mathrm{~kb}$ and $3.8 \mathrm{~kb}$, respectively, whereas that for the control strain is $1.8 \mathrm{~kb}$ (Figure 23). 
(a)

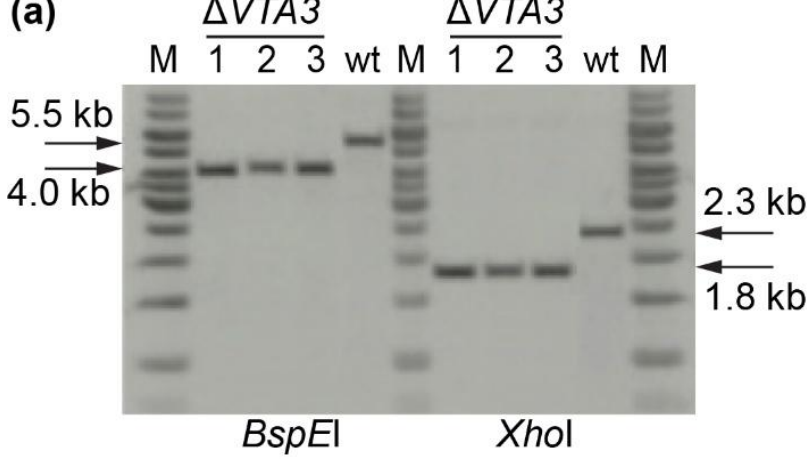

(b)

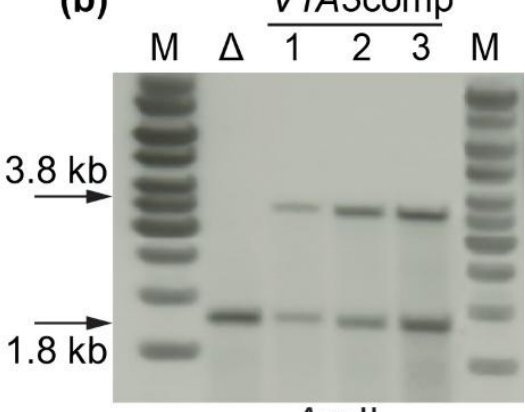

Avall

Figure 23. Confirmation of deletion and complementation strain of VTA3 in V. dahliae.

(a) Confirmation of deletion strains by Southern hybridization with two different restriction enzymes. The probe is the 3' flanking region. Genomic DNA of the wild-type was used as a control. (b) Confirmation of complementation strains by Southern hybridization with two different restriction enzymes. Genomic DNA of the VTA3 deletion strain was used as a control. The probe and sizes of expected fragments are indicated. The name of strains, restriction enzymes, and putative sizes are shown.

\subsubsection{Som1 promotes adhesion in V. dahliae}

The functions of Som1 and Vta3 in V. dahliae was studied by comparing the phenotype of deletion strains with that of wild-type and complementation strains. Specifically, the function of Som1 and Vta3 in hyphal clumping, biomass formation, adhesion, virulence, root penetration, conidia and microsclerotia formation, hyphal development, and oxidative stress response was investigated.

\subsubsection{Som1 is necessary for hyphal clumping and suppresses biomass formation}

The biomass of filamentous fungi in liquid medium is antagonistic to flocculation or hyphal clumping (Guebel \& Nudel, 1994). Our results show that Som1 is required for adhesion in $V$. dahliae (Figure 24). Therefore, the hyphal clumping and biomass of the SOM1 deletion strain might be affected. To test this hypothesis, the same number of spores of the wild-type, SOM1 deletion, and complementation strains was grown in $50 \mathrm{ml}$ liquid PDM on a shaker at $120 \mathrm{rpm}$ at $25^{\circ} \mathrm{C}$ for seven days. Hyphal ball formation and biomass production were observed. As seen in Figure 24, the SOM1 deletion strain produced smaller hyphal balls compared to JR2-wt and complementation strains. The size of hyphal balls of the SOM1 deletion strain was $80 \%$ smaller compared to the wild-type and complementation (Figure 24). The size of hyphal balls in deletion strain of SOM1 
did not increase during growth, whereas that of wild-type and complementation strain was continuously enlarged. Additionally, more hyphal balls were observed in the SOM1 deletion strain compared to the wild-type and complementation strains (Figure 24).

(a)
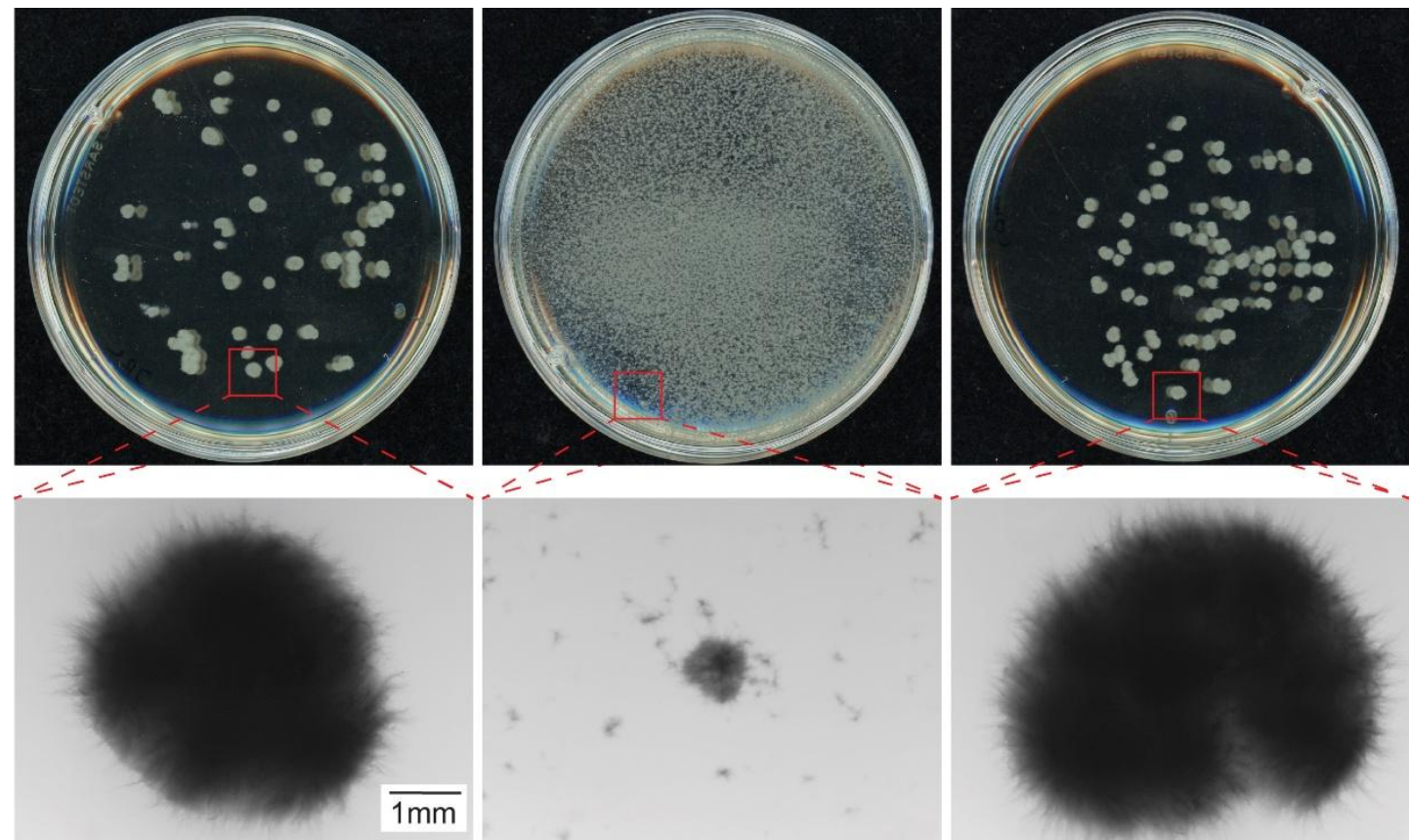

(b)

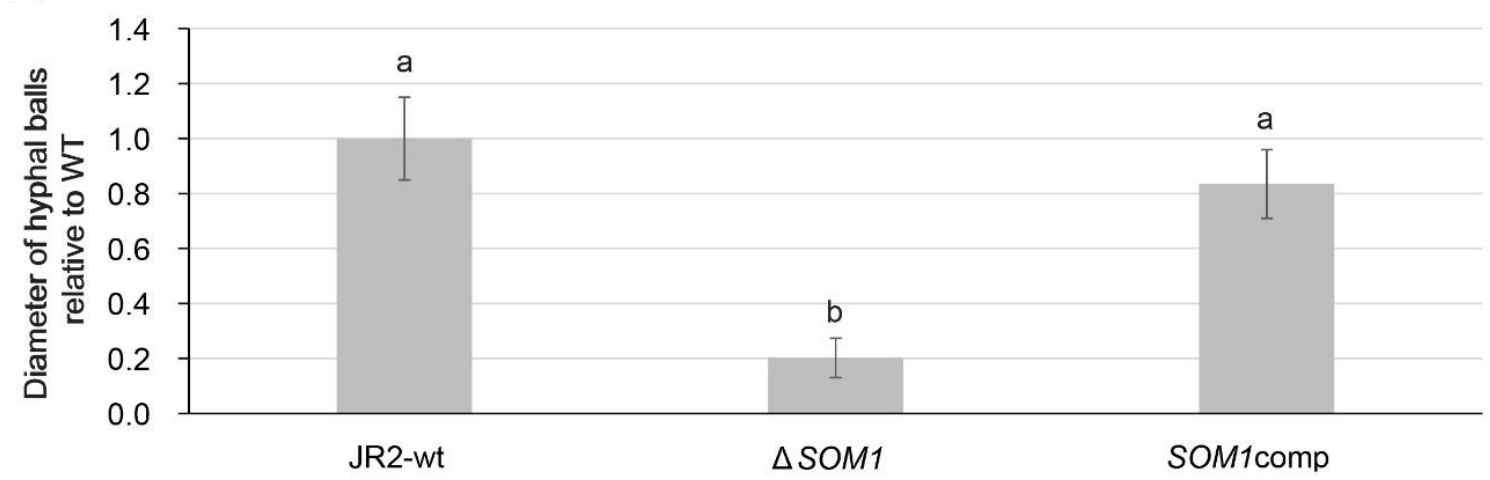

Figure 24. Som 1 is required for $\boldsymbol{V}$. dahliae hyphal clumping. (a) The same number of spores of JR2-wt, SOM1 deletion and complementation strains was grown in liquid PDM on a shaker at $25^{\circ} \mathrm{C}$ for seven days. The diameter of hyphal balls was observed. (b) Diagram of hyphal ball diameter. Experiments were performed in triplicate. Twenty hyphal balls were observed for each repetition. Mean values and standard deviation are presented. The letters $a$ and $b$ indicate groups which are significantly different as calculated by Tukey-Kramer multiple comparison procedures, $\alpha=0.01$. The scale bar is shown.

Next, the biomass of the SOM1 deletion strain was measured (Figure 25). As shown in Figure 25, the biomass of the SOM1 deletion strain was dramatically increased by $50 \%$ when compared to the wild-type and complementation strains 
after 7 dpi (Figure 25). The biomass of the deletion strain of SOM1 is continuously increased whereas that of the wild-type and complementation strains is unchanged after seven days. Taken together, the data suggest that Som1 is required for hyphal clumping and suppression of biomass production in $\mathrm{V}$. dahliae.

(a)

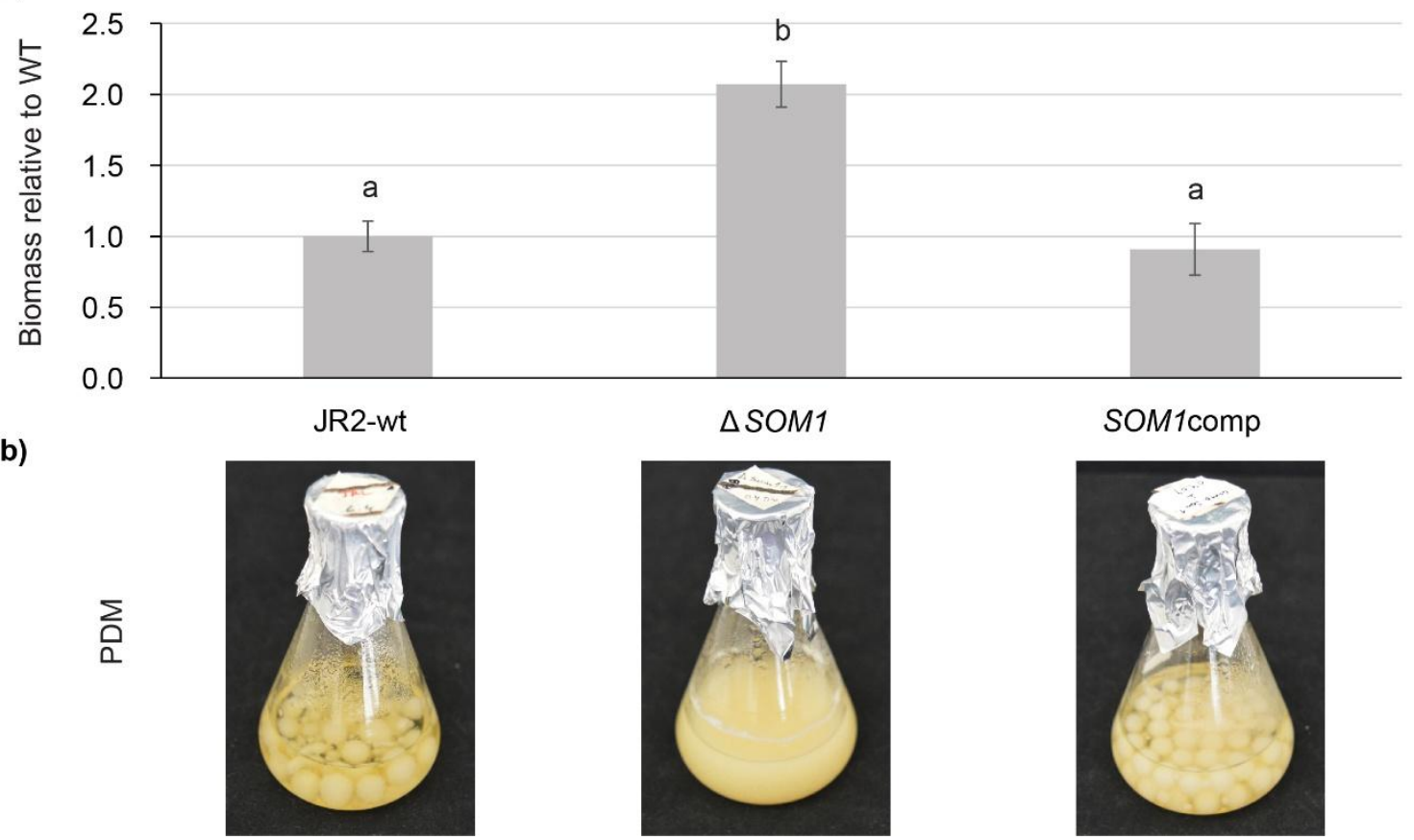

Figure 25. Som1 suppresses $\boldsymbol{V}$. dahliae biomass formation. The same number of spores of JR2-wt, SOM1 deletion and complementation strains was grown in liquid PDM on a shaker at $25^{\circ} \mathrm{C}$ for seven days. (a) The diagram of biomasses relative to the wildtype. (b) Picture of the culture after seven days of growth. Experiments were performed in triplicate. Mean values and standard deviation are presented. The letter $a$ and $b$ indicate groups which are significant different as calculated by Tukey-Kramer multiple comparison procedures, $\alpha=0.01$.

\subsubsection{Som 1 is needed for adherence on abiotic surfaces}

As Som1 rescued the non-adhesive phenotype of yeast. It might also play an important role in adhesion of Verticillium. Adhesion was tested using two different surfaces polystyrene plates and GelBond films.

To test adhesion to polystyrene plates, the wild-type, SOM1 deletion and complementation strains were incubated in liquid SXM in a polystyrene plate for seven days at $25^{\circ} \mathrm{C}$ and then the plate was washed with water. As seen in Figure 26, the SOM1 deletion strain was easily washed off the polystyrene, whereas the wild-type and complementation strains remained bound in the plates (Figure 26). 


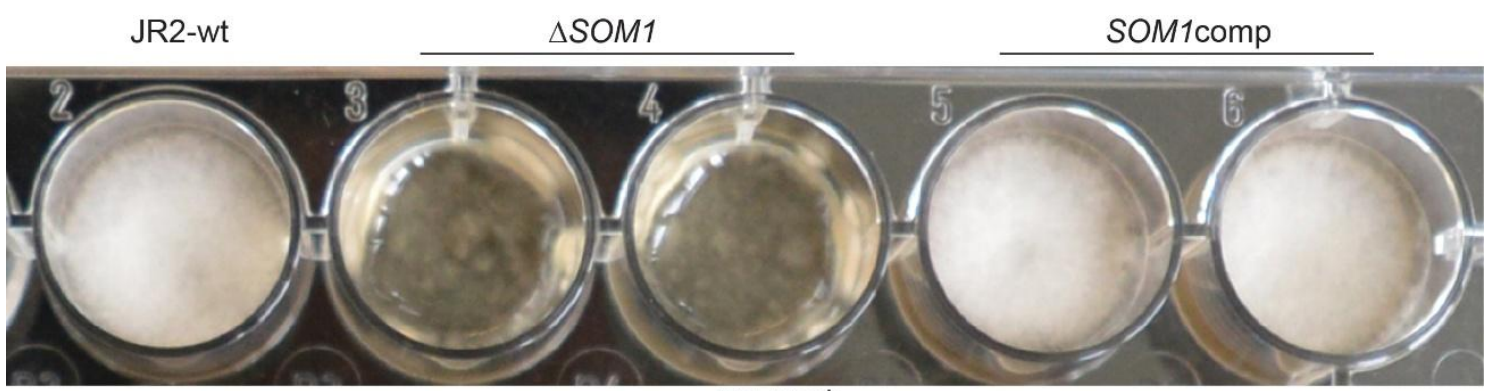

pre-wash

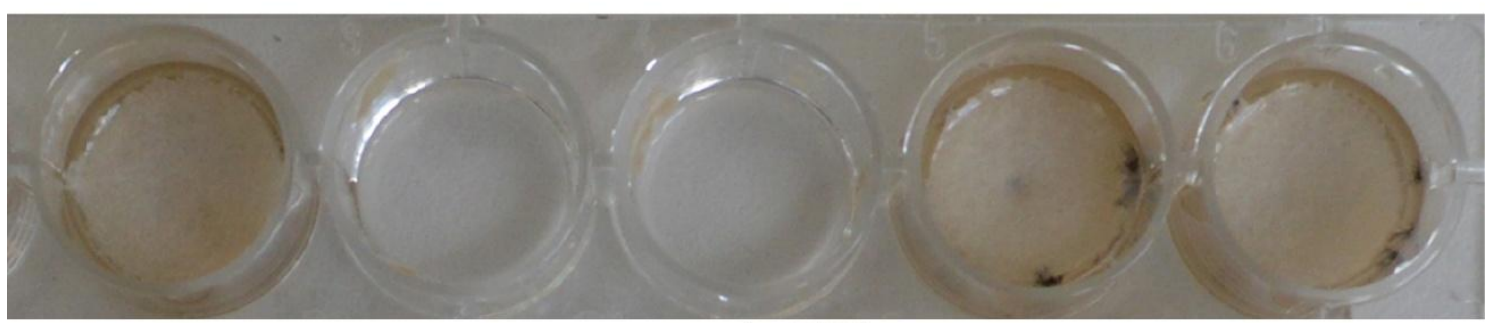

post-washed

Figure 26. Som 1 is necessary for adhesion of $V$. dahliae on polystyrene plates. The JR2-wt, SOM1 deletion and complementation strains were grown on polystyrene plates containing liquid SXM for seven days at $25^{\circ} \mathrm{C}$. The wells were washed with water. All experiments were performed in triplicates.

The adhesion of Verticillium to GelBond films was tested. The same number of spores was inoculated on Gelbond films and then incubated the membrane in a humid chamber. After two days, the GelBond was washed with water and adhering strains were observed by microscopy. The results in Figure 27 show that the SOM1 deletion strain can grow like the wild-type, but it is unable to adhere to the GelBond film. It was easily washed off when adding some drops of water. Many hyphae were detected in the wild-type and complementation strains but rarely any hyphae were seen in the SOM1 deletion strain (Figure 27). 

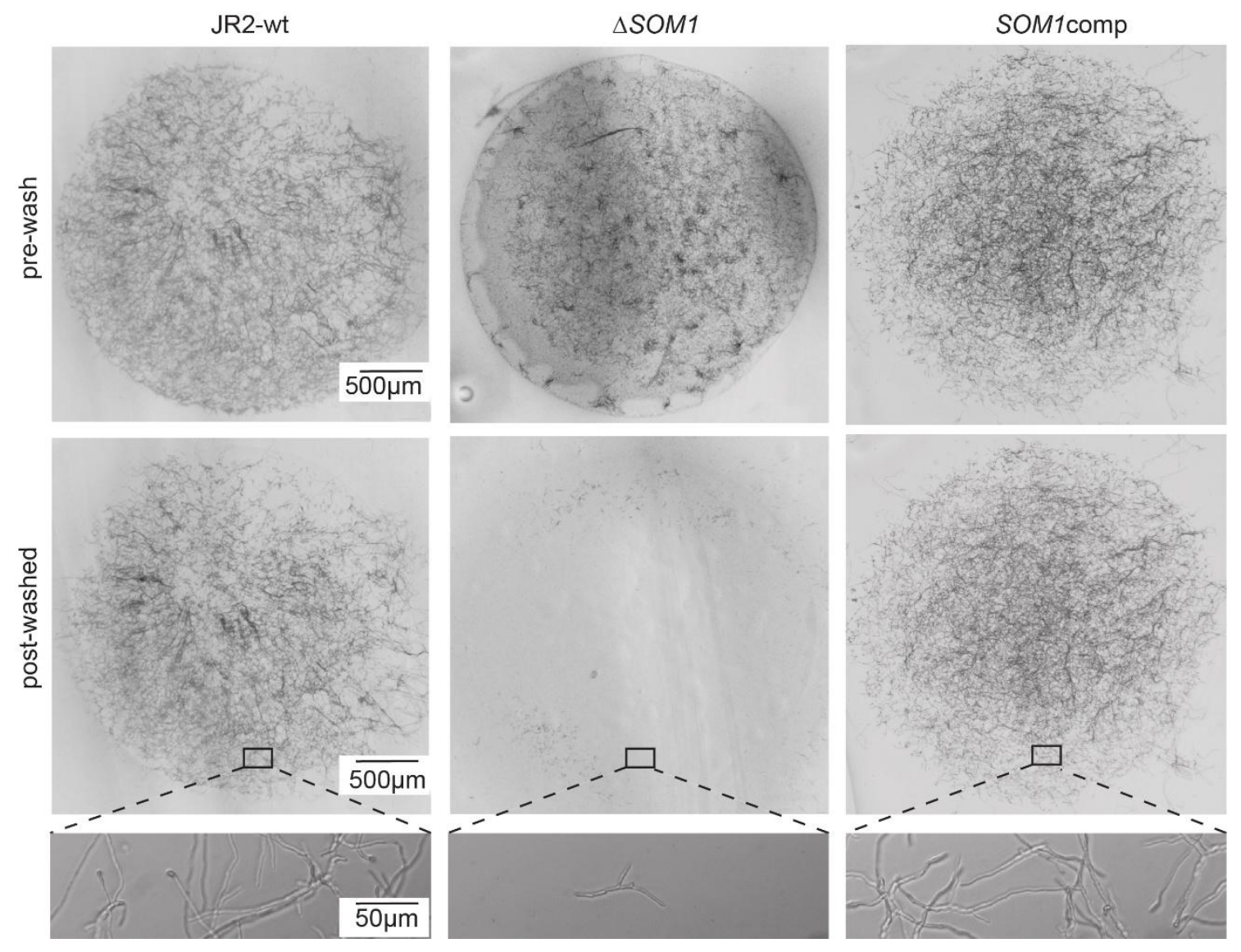

Figure 27. Som 1 is necessary for $\boldsymbol{V}$. dahliae adhesion to GelBond film. The same number of spores of the JR2-wt, SOM1 deletion and complementation strains in liquid MM (1/10 of concentration) were placed on hydrophobic surfaces of the GelBond film and incubated at $25^{\circ} \mathrm{C}$ in the dark for two days. Hydrophobic surfaces of the GelBond film were washed with water and further processed under the microscope. All experiments were performed in triplicates. Scale bars are shown.

\subsubsection{Som 1 and $V t a 3$ are required for pathogenicity}

Verticillium dahliae is known to cause wilting disease in more than 200 plant species including tomato plants (Pegg \& Brady, 2002; Berlanger \& Powelson, 2005). It was reported to cause also wilt disease in Arabidopsis thaliana (Wilhelm, 1955; Pegg \& Brady, 2002). V. dahliae infects plants via the roots, which requires root penetration and colonisation as initial steps of infection. Therefore, the effect of Som1 and $\mathrm{Vta} 3$ on virulence was examined in tomato plants and on $A$. thaliana roots. 


\subsubsection{Som1 and Vta3 are involved in fungal pathogenicity}

Ten days old tomato plants were used to test the virulence of Som1 and Vta3. The disease symptoms of plants infected by the deletion strains of SOM1 and VTA3 were compared to the wild-type and their complementation strains up to $35 \mathrm{dpi}$ (Figure 28).

(a)
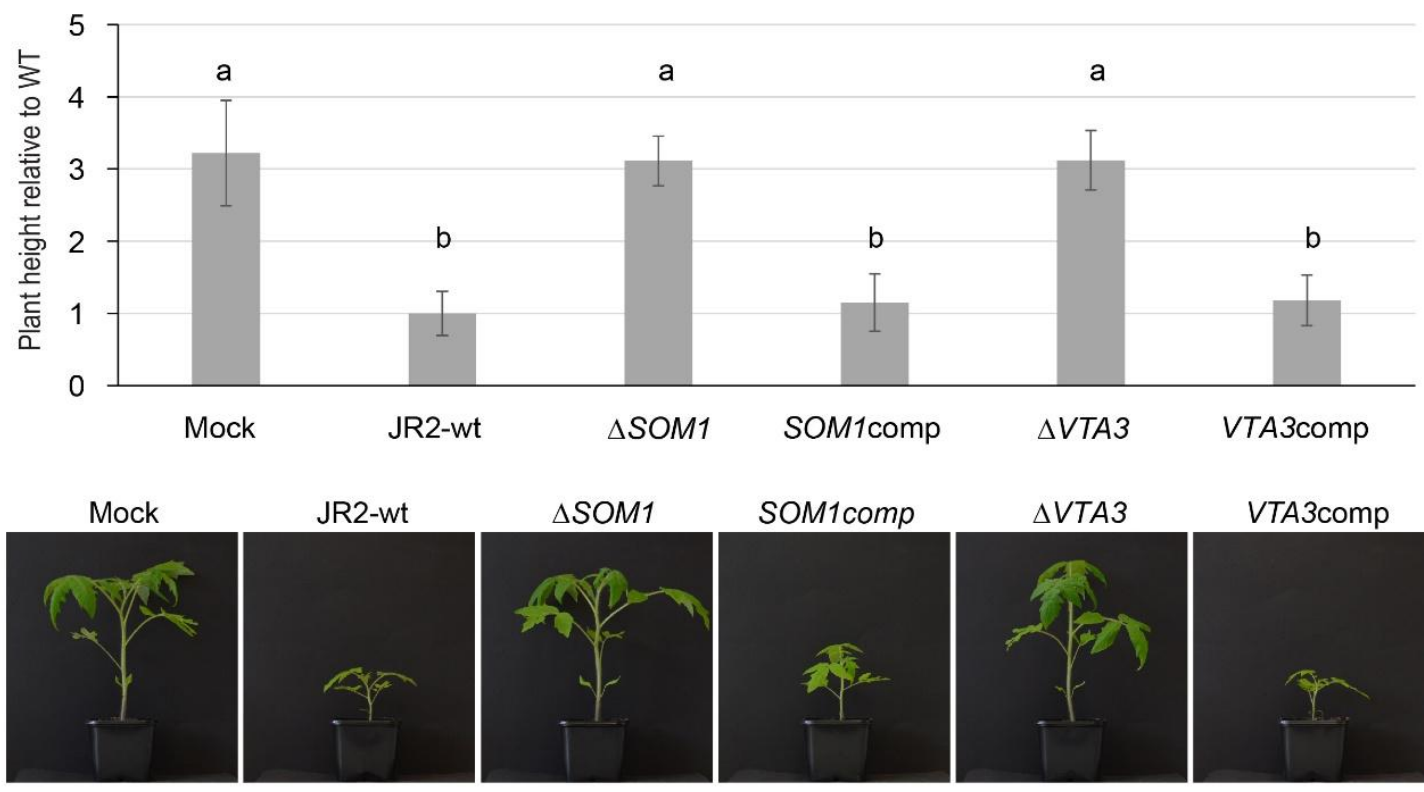

(b)

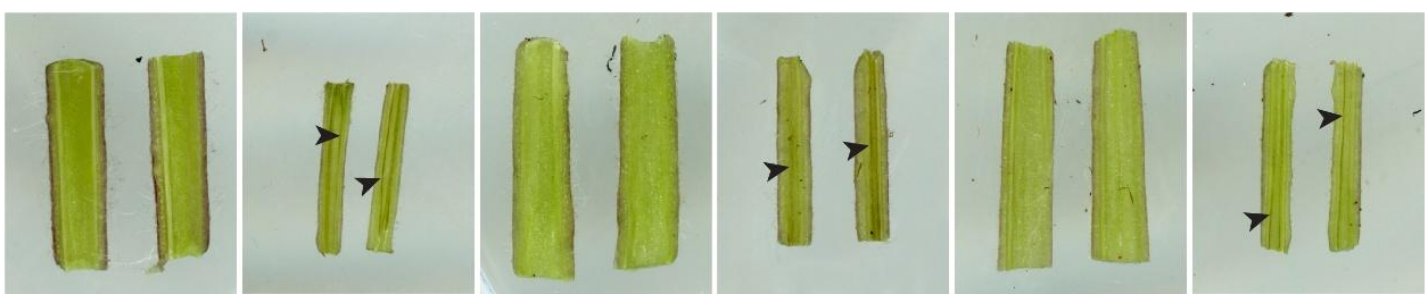

Figure 28. Som 1 and Vta3 are required for introduction of disease symptoms in tomatoes. Ten days old tomatoes were infected with the same number of spores of indicated strains or remained uninfected (mock). The plants were incubated in the climate chamber under $16 \mathrm{~h}$ light: $8 \mathrm{~h}$ dark at $22-25^{\circ} \mathrm{C}$. The disease symptoms were assessed at 35 days post infection (dpi). Infection experiments were performed with 16 single plants for each fungal strain and independently repeated twice. (a) The plant height was measured. The mean values and standard deviations are indicated. The letters $a$ and $b$ show groups which are significantly different as calculated by Tukey-Kramer multiple comparison procedure $\alpha=0.01$. Representative plants are shown. (b) Black threads in infected stems were observed. The arrowhead indicates black veins.

The wild-type and the complementation strains initiated severe stunting and leaf chlorosis in infected tomato plants which were accompanied by discoloration of the vascular system (Figure 28). In contrast, plants infected with the SOM1 or VTA3 
deletion strains showed no disease symptoms and they were indistinguishable from uninfected plants (Figure 28). The height of plants infected by the deletion strains of SOM1 and VTA3 is significantly higher than that of plants infected by the wild-type and complementation strains (Figure 28a). No black veins in stems were found in the plants infected by deletion strains (Figure 28b).

The presence of the pathogenic fungus in the plant was examined by its re-isolation from hypocotyls and quantification of the fungal DNA from the total plant DNA by real-time PCR with OLG primer pairs (Eynck et al., 2007; Timpner et al., 2013). The fungal pathogen was successfully re-isolated from hypocotyls of the plants infected with the wild-type and complementation strains, but not from the plants infected by SOM1 and VTA3 deletion strains (Figure 29a). The concentration of fungal DNA in the total plant DNA was also examined (Figure 29b). As demonstrated in Figure 29b, fungal DNA was not detected in plants infected with the SOM1 deletion strain, whereas fungal DNA was reduced by $82 \%$ in plants infected with the VTA3 deletion strain when compared to plants infected with wild-type. Similar results were obtained for fungal DNA extracted either from the root or the hypocotyls.

(a)

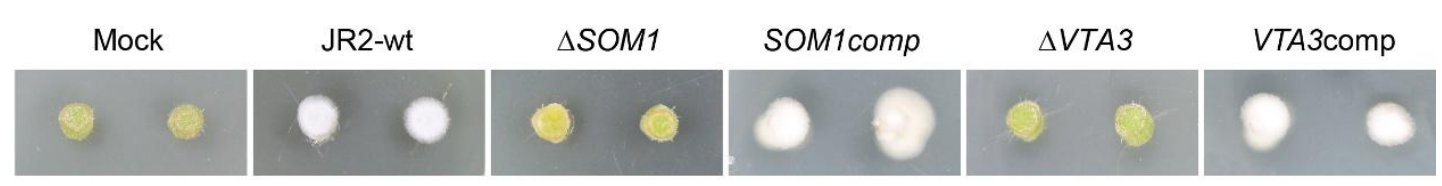

(b)

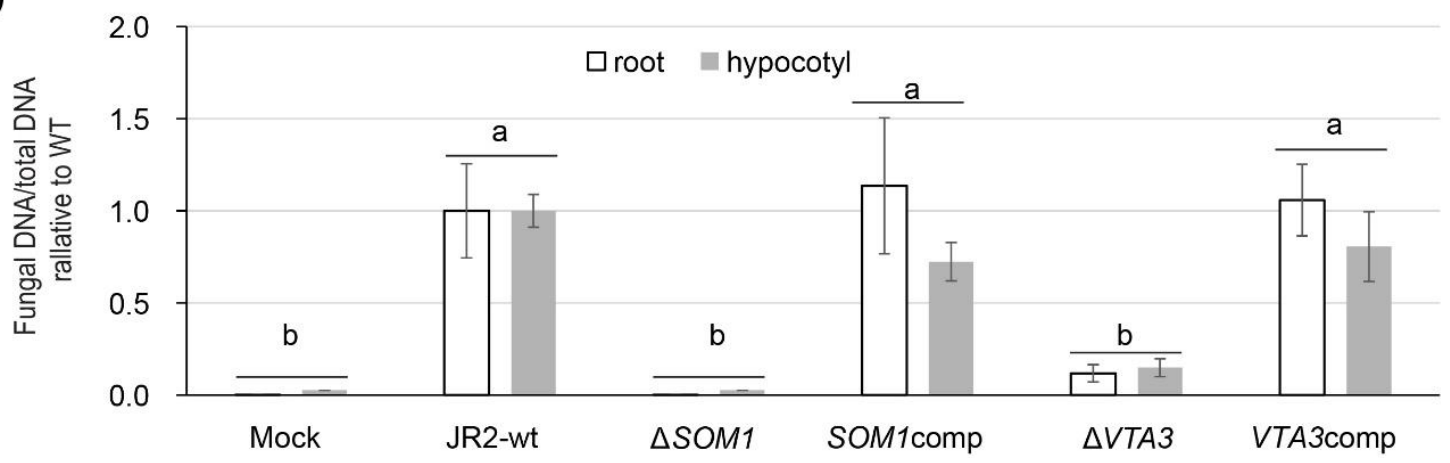

Figure 29. V. dahliae Som 1 and Vta3 are essential for plant infection. (a) Fungal reisolation from hypocotyl was examined. The surface-sterilized hypocotyls were placed on PDM plates containing cefotaxime and chloramphenicol and incubated for seven days. (b) The total DNA from roots and stems was purified. The relative amount of fungal DNA from the total plant DNA was quantified by RT-PCR using the OLG primer pairs (Eynck et al., 2007). This experiment was performed with four repetitions. The mean values and standard deviations are indicated. The letters $a$ and $b$ show groups which are significantly different as calculated by Tukey-Kramer multiple comparison procedure $\alpha=0.01$. 


\subsubsection{Fungal Som1 and Vta3 are required for sequential steps of plant root penetration and colonisation}

Verticillium species belong to soil-borne ascomycete fungi which enter the host plant via the roots. Both, the SOM1 and VTA3, deletion strains are avirulent strains, therefore, Arabidopsis root infection was examined to find out why the deletion strains of SOM1 and VTA3 are avirulent. Three weeks old $A$. thaliana roots were infected with the same number of spores of the wild-type, SOM1, or VTA3 deletion strains expressing free GFP under the control of the gdpA promoter. The results shown in Figure 30 , indicate that $24 \mathrm{~h}$ post inoculation (hpi), the germination of indicated strains is indistinguishable. The VTA3 deletion strain shows the same potential as wild-type for the initial invasion of plant roots until $48 \mathrm{hpi}$, whereas the SOM1 deletion strain is unable to form hyphopodia on the root surface. The plant root infection at $120 \mathrm{hpi}$ revealed that the SOM1 deletion strain is unable to penetrate the host and proliferate on the root surface. Hyphae of the SOM1 deletion strain grow between root hairs but not on the root surface, whereas the VTA3 deletion strain penetrates but is unable to proliferate to the same extend as the wild-type strain (Figure 30).

Hyphal-root interactions were further investigated by scanning electron microscopy at $72 \mathrm{hpi}$. The results shown in Figure 31, indicate that hyphae of the JR2-wt well colonise and can tightly bind to the root surface. Hyphae of the JR2wt either enter plant roots directly or form hyphopodia, whereas SOM1 and VTA3 deletion strains are impaired in colonisation of root surfaces. The strains, carrying the deletion of VTA3, can still develop on root surfaces and forms hyphopodia, but its colonisation is reduced when compared to the wild-type strain (Figure 31). 

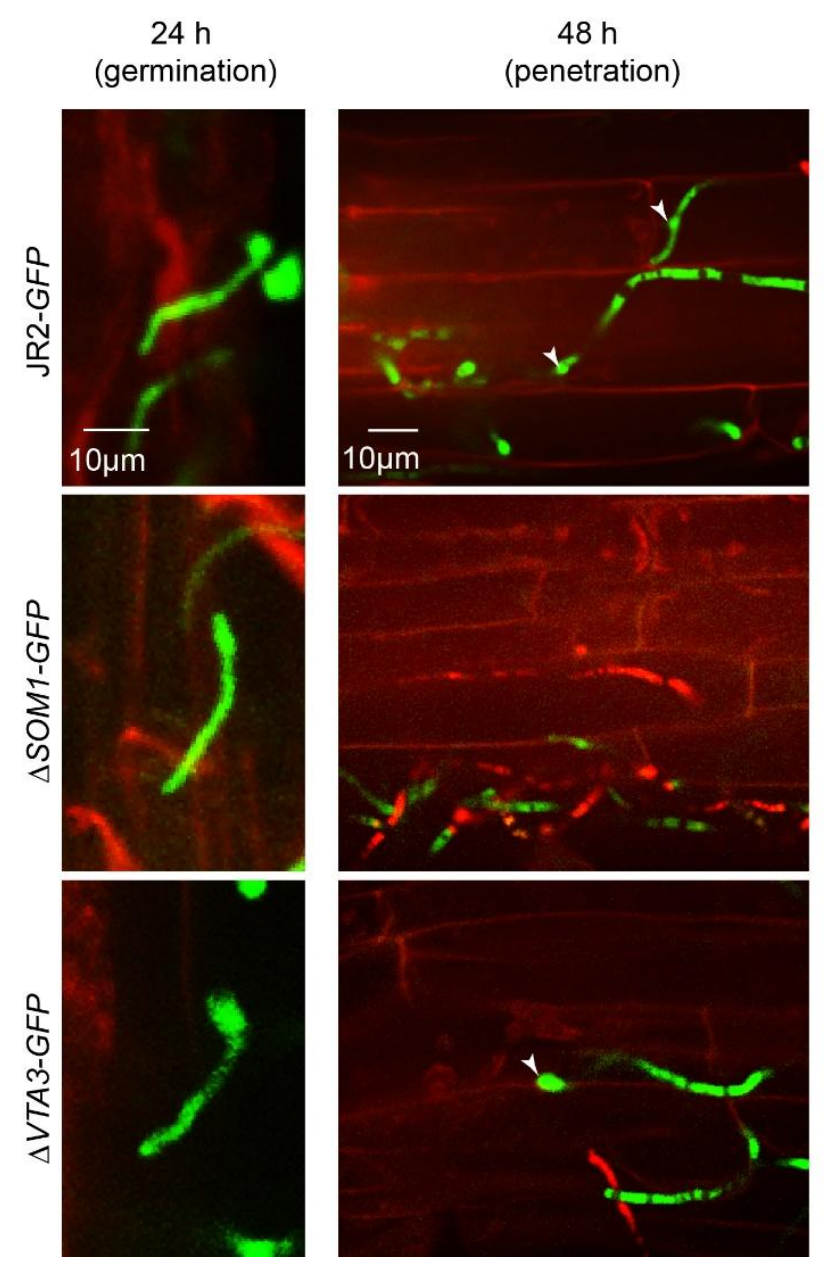

$48 \mathrm{~h}$ (penetration)

Figure 30
$120 \mathrm{~h}$ (colonization)

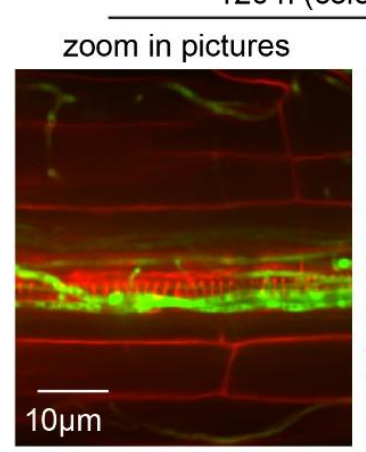

overview pictures
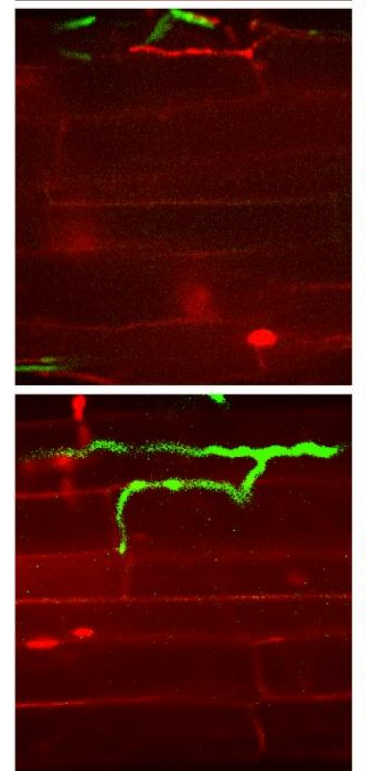

$10 \mu \mathrm{m}$
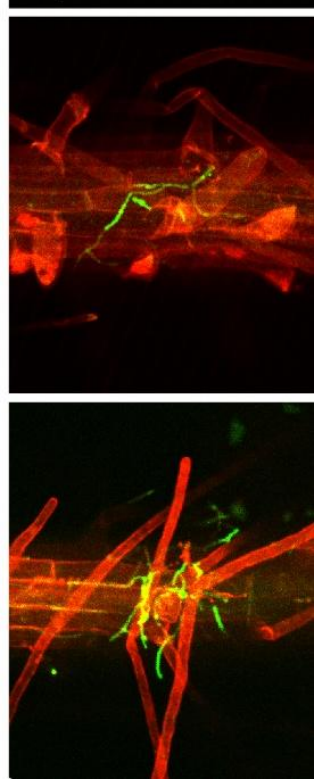

penetration and root colonisation. Three weeks old Arabidopsis thaliana plants were infected with the same number of spores of JR2-wt (JR2-GFP), SOM1 deletion ( $\triangle S O M 1$ GFP), and VTA3 deletion ( $\triangle V T A 3-G F P$ ) strains expressing free GFP. The germination, penetration, and colonisation of fungal hyphae on the root surface were observed after $24 \mathrm{~h}$, $48 \mathrm{~h}$, and $120 \mathrm{~h}$. The experiment was performed with five plants for each fungal strain. The arrowheads indicate the penetration points. Scale bars are represented. The SOM1 deletion strain is hardly detectable on plant roots. Only short fragments of hyphae could be detected on plant roots which were infected with the SOM1 deletion strain (Figure 31). In summary, Som1 and Vta3 represent control factors for sequential steps of fungal penetration and colonisation on plant root surfaces. 


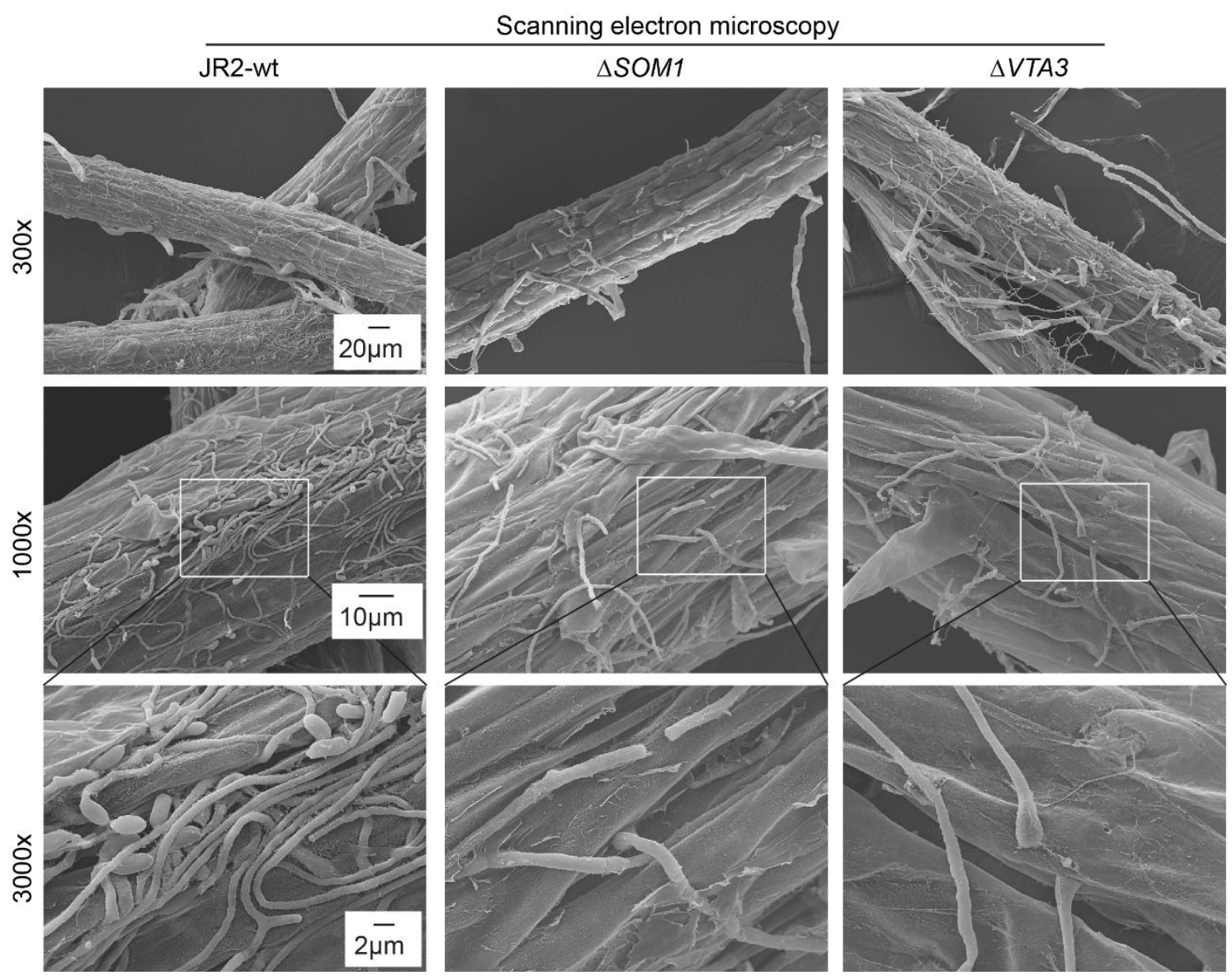

Figure 31. Som1 and Vta3 are required for successful root colonisation. Three weeks old Arabidopsis thaliana plants were infected with the same number of spores of JR2-wt, SOM1 deletion, and VTA3 deletion strains. The root infection was analysed by scanning electron microscopy at $72 \mathrm{hpi}$. Scale bars are represented.

\subsubsection{Som1 and Vta3 support conidia and microsclerotia formation}

Conidia and microsclerotia are important structures for the plant pathogen V. dahliae. Conidia are developed on forming phialides (Wilhelm, 1955; Schnathorst, 1982; Xiao et al., 1998; Pegg \& Brady, 2002). Each phialide has a mass of spores which is called a conidiospore cluster in the following. Conidia play a major role in the distribution of the pathogen in all plant cells and spreading fungus through water, whereas microsclerotia are important for the distribution of $V$. dahliae worldwide and for survival of fungi in the soil for up to 15 years. The effect of Som1 and Vta3 on conidia and microsclerotia formation was therefore further analysed. 


\subsubsection{Som1 and Vta3 promote conidia formation}

The formation of spores in liquid medium and conidiospore clusters on agar plates was examined. Liquid medium was inoculated with the same number of spores and grown for seven days. The spores of each strain were separately counted and plated in Figure 32. The number of spores in the SOM1 and VTA3 deletion strains was significantly decreased by $92 \%$ and $59 \%$, respectively (Figure $32 a$ ).

Conidia are formed at a specialised structure, called the conidiophore. Each conidiophore produces several phialides in which a number of conidia develop. This unit of conidia on a phialide, we called conidiospore cluster. The formation of these structures was tested on CDM plates (Figure 32b). The same number of spores of indicated strains was dropped on CDM plates and grown in the dark for three days. The formation of conidiospore cluster was observed with a binocular microscope. As seen in Figure 32b, the SOM1 deletion strain has fewer conidiospore clusters than JR2-wt and complementation strains, whereas the number of conidiospore clusters was indistinguishable between the VTA3 deletion strain and the wild-type. However, $60 \%$ of the conidiospore clusters in VTA3 deletion strain had oval shapes (data not shown), whereas they were round in the wild-type and complementation strains (Figure 32b).

(a)

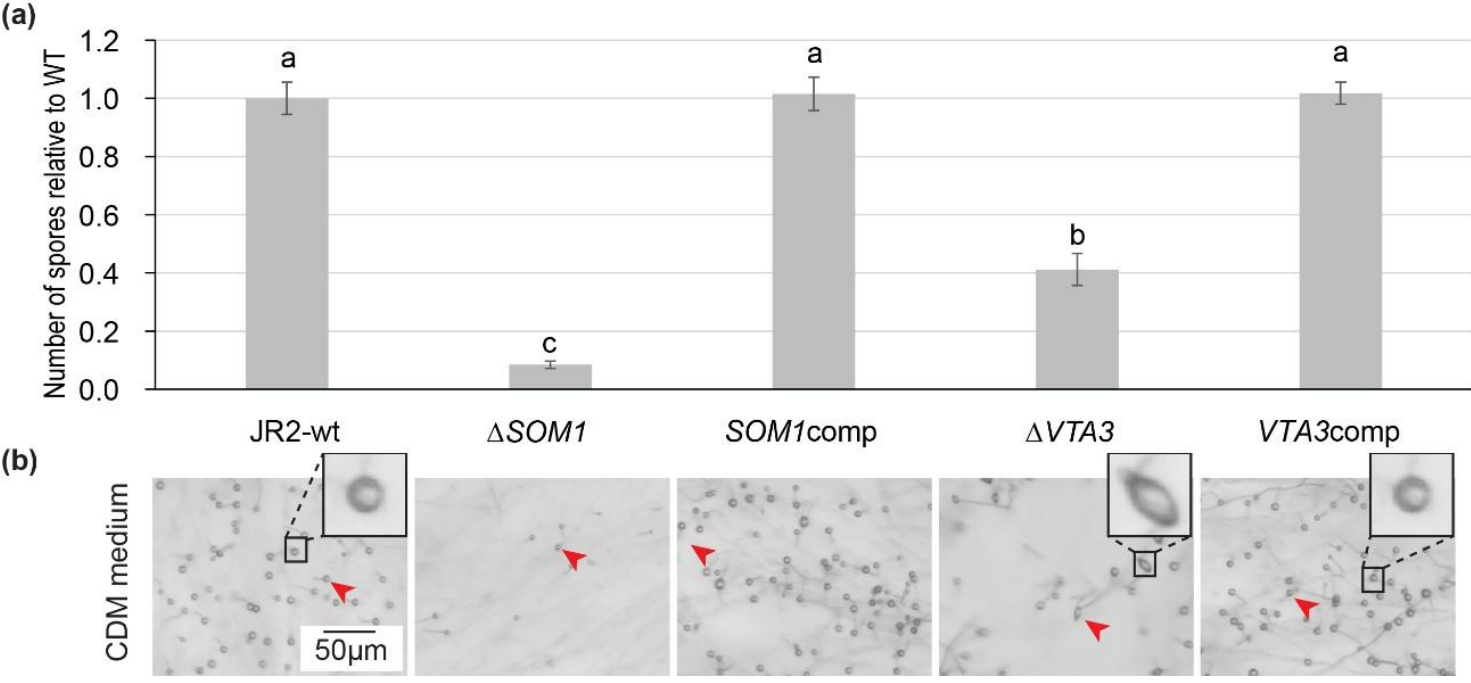

Figure 32. Som1 and Vta3 promote conidia formation. (a) The same number of spores of JR2-wt, SOM1 deletion and complementation, and VTA3 deletion and complementation strains were cultivated in $50 \mathrm{ml}$ liquid SXM on a shaker at $25^{\circ} \mathrm{C}$ for seven days. The spore numbers were counted. The mean values and standard deviations of triplicates are shown. (b) The formation of conidiospore clusters on agar plates was examined. The same spore numbers of indicated strains were dropped on CDM plates and grown at $25^{\circ} \mathrm{C}$ for seven days. The conidiospore cluster density was investigated. The arrowhead indicates a conidiophore. Scale bars are shown. 


\subsubsection{Som1 and Vta3 control microsclerotia formation}

Verticillium pathogens develop resting structures as melanized microsclerotia which help pathogens to persist in the soil for a decade even in the absence of plant hosts (Wilhelm, 1955; Tran et al., 2013). The effects of Som1 and Vta3 on microsclerotia formation were examined on CDM plates containing $3 \%$ cellulose (Figure 33 ). The number of microsclerotia on the surface and in the agar was counted. The data presented in Figure 33a suggest that the VTA3 deletion strain produces $60 \%$ less microsclerotia than the wild-type and the complementation strains, whereas the SOM1 deletion strain is unable to form microsclerotia after seven days (Figure 33a).

Microsclerotia formation of JR2-wt and SOM1 deletion strains was further investigated by scanning electron microscopy (Figure 33b). No microsclerotia structures were detected in the SOM1 deletion strain, whereas they are easily found in the JR2-wt strain. 
(a)
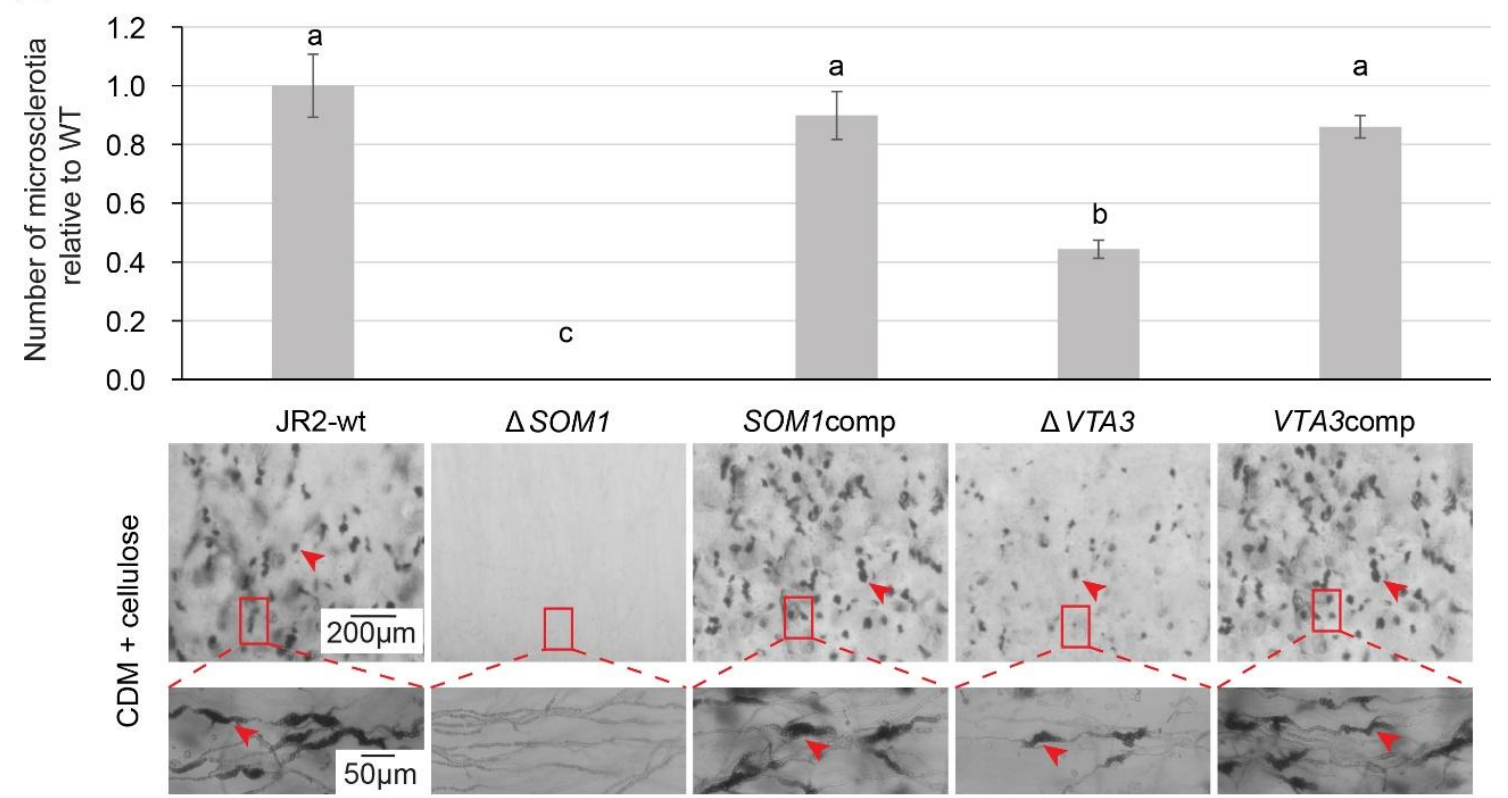

(b)

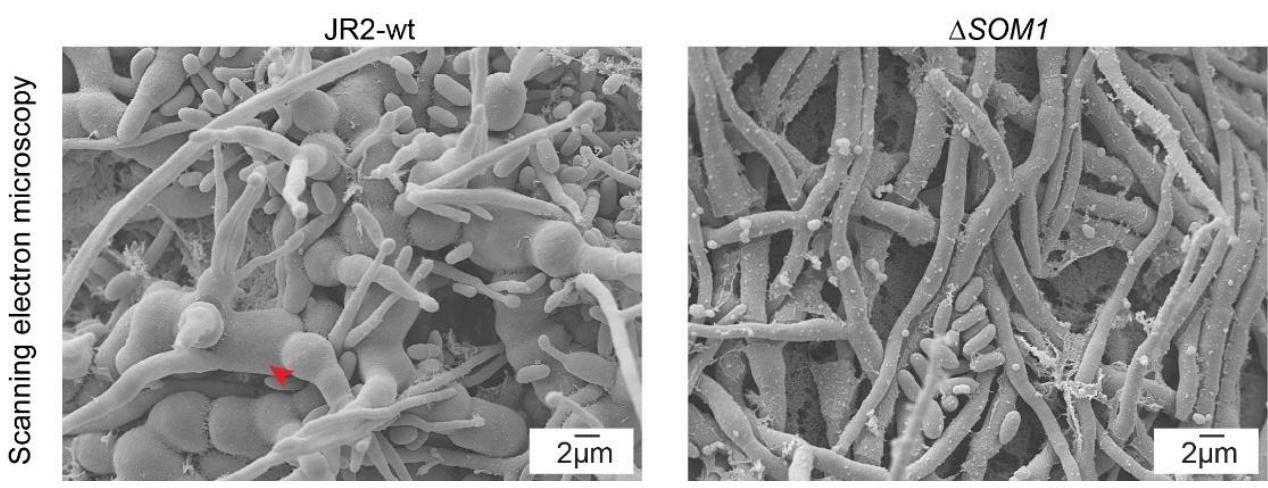

Figure 33. Som 1 and Vta3 control microsclerotia formation. (a) Spores of the indicated strains were dropped on CDM plates containing 3\% cellulose and incubated in the dark. The microsclerotia formation was observed by binocular microscopy. The arrowhead shows a microsclerotium. The number of microsclerotia was counted. Experiments were performed in triplicate. The mean values and standard deviation are shown. The letters a-c indicate the groups which are significant different as calculated by Tukey-Kramer multiple comparison procedures, $\alpha=0.01$. (b) The formation of microsclerotia of JR2-wt and the SOM1 deletion strain at five days on SXM plates was examined by scanning electron microscopy. Scale bars are shown.

The formation of microsclerotia in the overexpression strain of SOM1 was examined, as seen in Figure 34. The data indicate that the $V$. dahliae overexpression strain of SOM1 produces microsclerotia earlier than the wild-type strain. The overexpression strain of SOM1 produced microsclerotia and melanin, seen black on PDA plates after seven days while the colony of the wild-type strain was white (Figure 34a). The number of microsclerotia formed on CDM plate by the SOM1 overexpression strain was also quantified. As seen in Figure 34b, the number of microsclerotia in this strain was significantly higher compared to the wild-type strain by 1.5 fold (Figure $34 b$ ). 
(a)

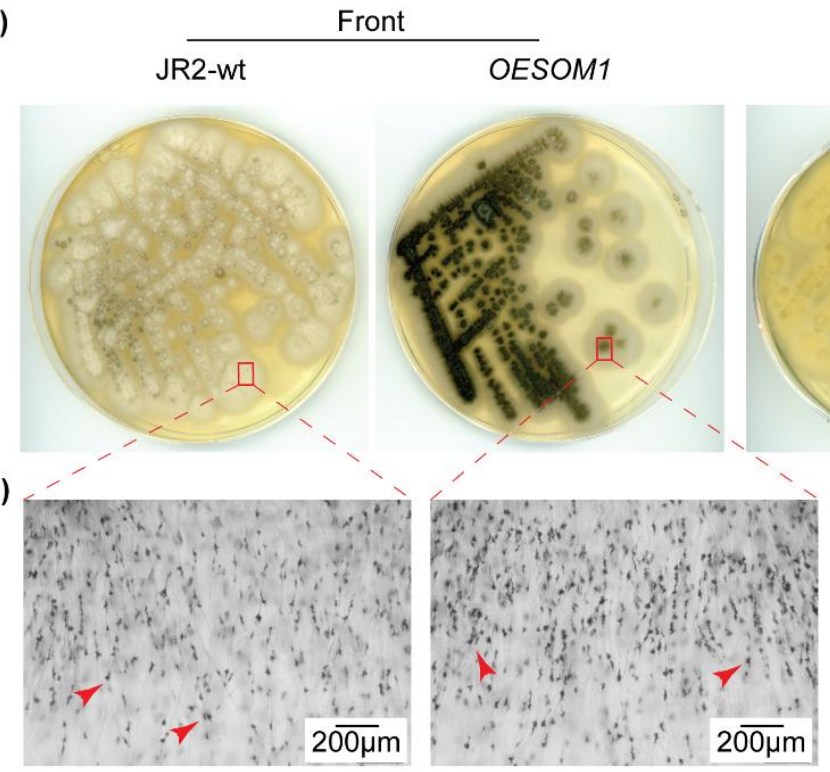

\begin{tabular}{ll}
\multicolumn{3}{c}{ Back } \\
JR2-wt & OESOM1
\end{tabular}

$200 \mu \mathrm{m}$

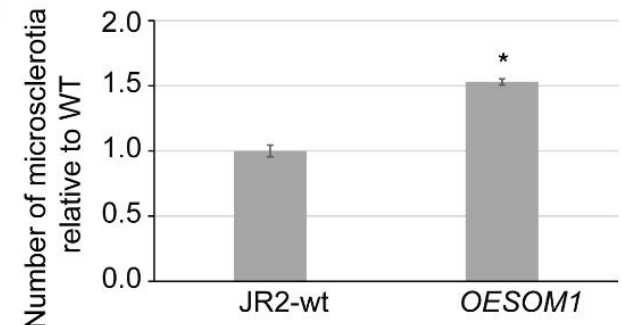

Figure 34. The overexpression of SOM1 enhances the number of microsclerotia. (a) The overexpression of SOM1 in V. dahliae JR2 shows earlier melanin production on PDA plates. The spores of the JR2-wt and the overexpression of SOM1 strains were spread on PDA plates and grown in the dark at $25^{\circ} \mathrm{C}$ for seven days. The formation of microsclerotia of the SOM1 overexpression was examined. (b) The same spore number of indicated strains was dropped on CDM plates containing $3 \%$ cellulose and grown in the dark at $25^{\circ} \mathrm{C}$ for seven days. The experiment was performed in triplicate. The microsclerotia number was counted after seven days. The mean values and standard deviations are shown. The asterisk (*) indicates a significant increase of microsclerotia in the SOM1 overexpression when compared to wild-type strains (Student's t-test, $p<0.05$ ). The arrowhead indicates a microsclerotium. Scale bars are displayed.

\subsubsection{Som1 and Vta3 antagonise in oxidative stress response}

Plants can generate reactive oxygen species (ROS) as hydrogen peroxide $\left(\mathrm{H}_{2} \mathrm{O}_{2}\right)$ to ward off invading microbial pathogens. Pathogenic fungi need to develop defense systems against the plant immune system. The ability of the SOM1 and VTA3 deletion strains to grow in the presence of exogenous $\mathrm{H}_{2} \mathrm{O}_{2}$ and menadione-induced stress was analysed by measuring the diameter of the inhibition zone around filter papers containing oxidative stress inducing agents (Figure 35). The inhibition zone of the SOM1 deletion strain was significantly increased, whereas that of the VTA3 deletion strain decreased when compared to wild-type in both stress tests (Figure 35). This result suggests that Som1 is required for an appropriate stress response, whereas Vta3 inhibits this reaction. 

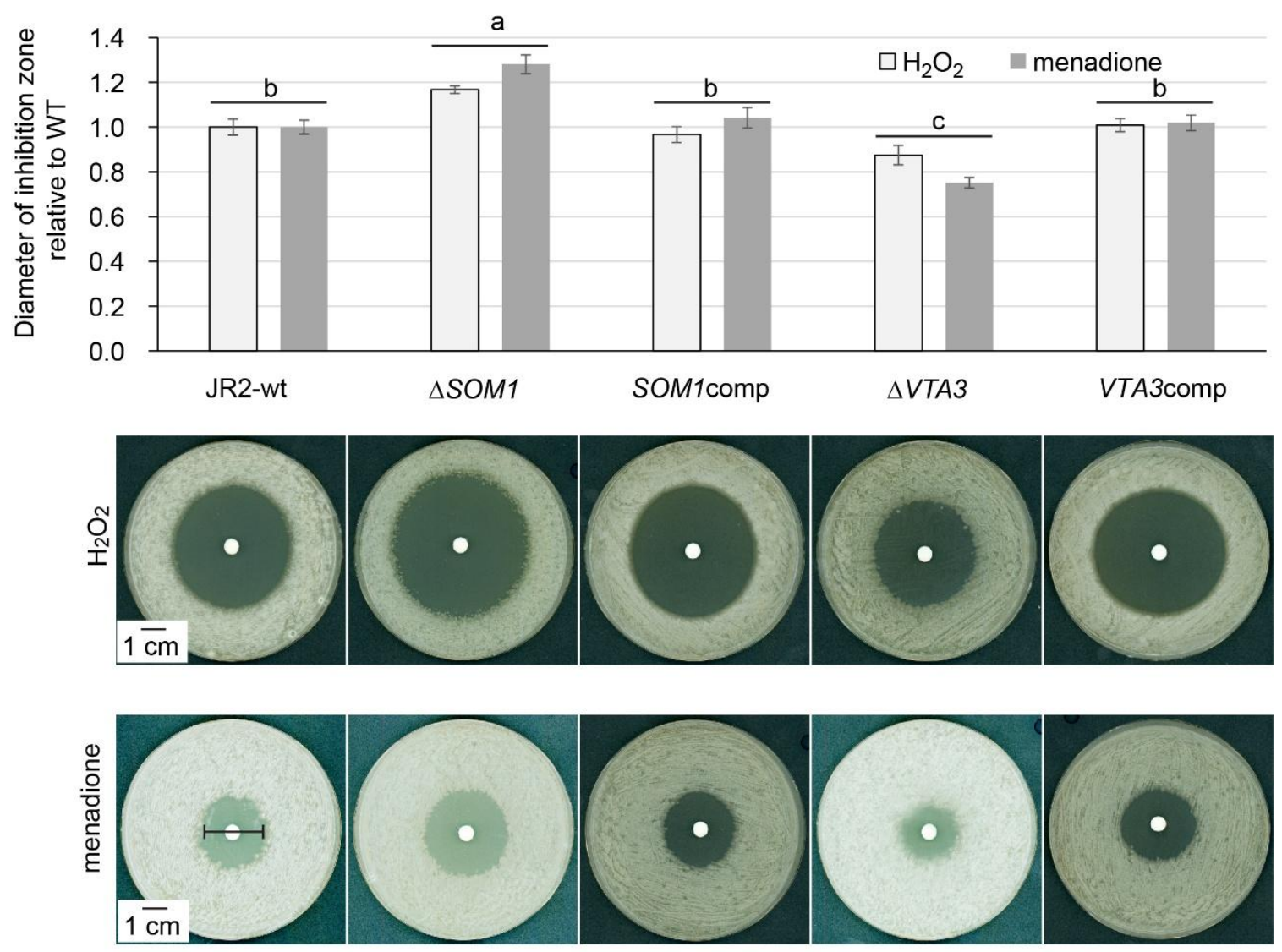

$\longmapsto$ indicates the diameter of the inhibition zone

Figure 35. Som1 and Vta3 antagonise in oxidative stress response. The same number of spores of JR2-wt, SOM1 deletion and complementation, and VTA3 deletion and complementation strains was spread on PDM plates and a filter paper disc containing $10 \mu \mathrm{H}_{2} \mathrm{O}_{2} 10 \%$ or menadione $100 \mu \mathrm{g} / \mu \mathrm{l}$ were placed in the center of plates. Plates were incubated in the dark for four days at $25^{\circ} \mathrm{C}$. The diameter of the inhibition zone was measured. The experiment was performed in triplicate. Mean values and standard deviations are shown. The letters a-c indicate groups which are significant different as calculated by Tukey-Kramer multiple comparison procedures, $\alpha=0.01$. Scale bars are represented.

\subsubsection{Som1 and Vta3 are needed for hyphal growth of $V$. dahliae on agar plates}

The growth of SOM1 and VTA3 deletion strains was examined in various media including different carbon source media (Figure 36). In general, both deletion strains of SOM1 and VTA3 grow significantly slower than the wild-type and complementation strains in all tested media (Figure 36). However, the growth of SOM1 and VTA3 deletion strain was similarly slower on glucose-containing medium, whereas significant differences in the growth of SOM1 deletion strain compared to VTA3 deletion strain was observed in all other carbon sources. Additionally, 
the growth of the SOM1 deletion strain depends on the carbon source. The deletion of SOM1 grows faster in glucose-containing medium, whereas it develops very slowly in the medium containing galactose (Figure 36). In summary, Som1 and Vta3 are essential for hyphal growth on agar plates and Som1 is required for growth in galactose-containing medium. 

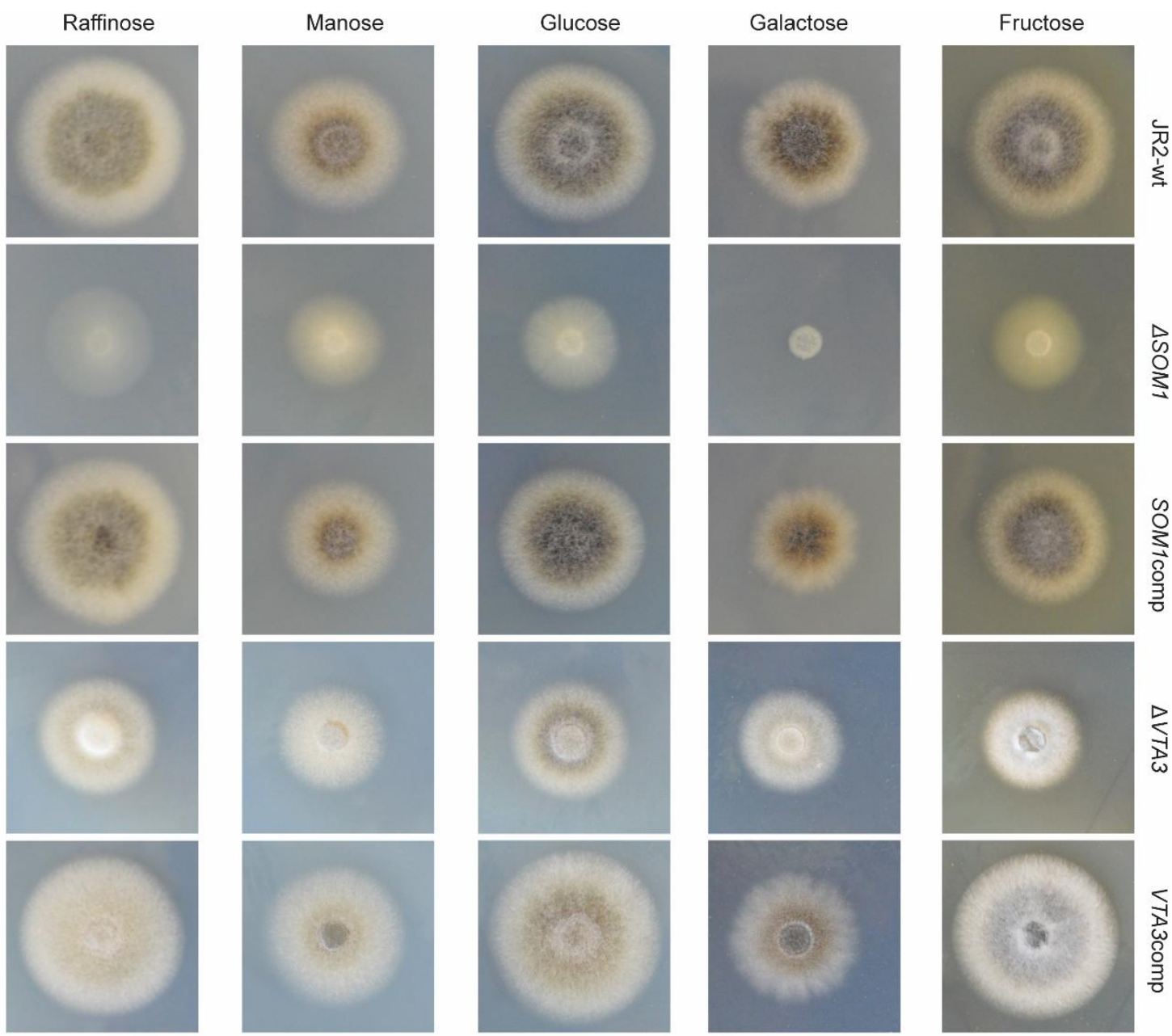

$\varnothing$ colony growth relative to WT

$\varnothing$ colony growth relative to WT

$\varnothing$ colony growth relative to WT

$\varnothing$ colony growth

$\varnothing$ colony growth relative to WT
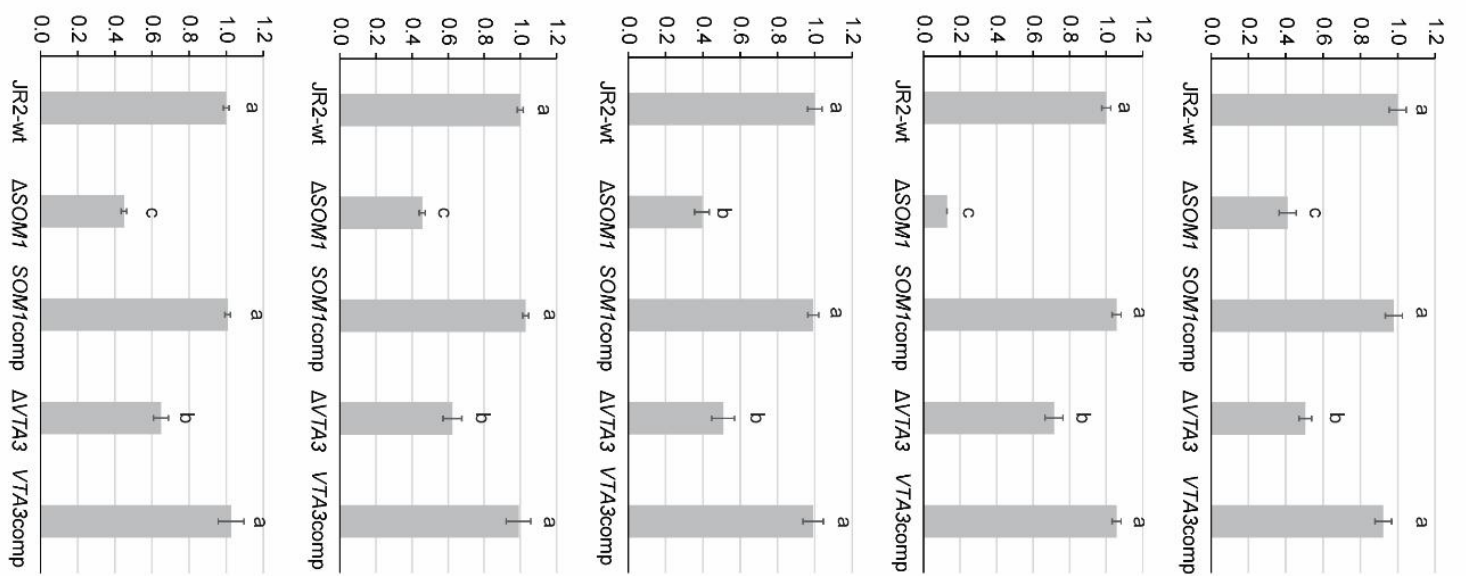

Figure 36. Som1 and Vta3 affect growth on different carbon sources. Czapek Dox agar was used for growth tests with different carbon sources as indicated (3\%). The same number of spores of indicated strains was dropped on agar plates and incubated in the dark at $25^{\circ} \mathrm{C}$. The diameter of the colony was measured after 11 days post inoculation. The mean values and standard deviations from three repetitions are displayed. The letters a-c are groups which are significantly different as calculated by Tukey-Kramer multiple comparison procedures, $\alpha=0.01$. 


\subsubsection{Som 1 is essential for hyphal development in $V$. dahliae}

Adhesion of cells to a surface and the clumping of hyphae in liquid medium might represent two aspects of hyphal development. For a more detailed analysis, the JR2-wt and SOM1 deletion and complementation strains were grown on agar plates and in liquid medium, and the hyphal development was further investigated.

The wild-type, deletion of SOM1, and complementation strains were grown on agar plates and in liquid SXM without shaking. The formation of aerial hyphae was investigated, as shown in Figure 37. Aerial hyphae are easily observed in the wild-type and complementation strains either on agar plates or in standing liquid SXM, whereas they are hardly detected in the SOM1 deletion strain (Figure 37).
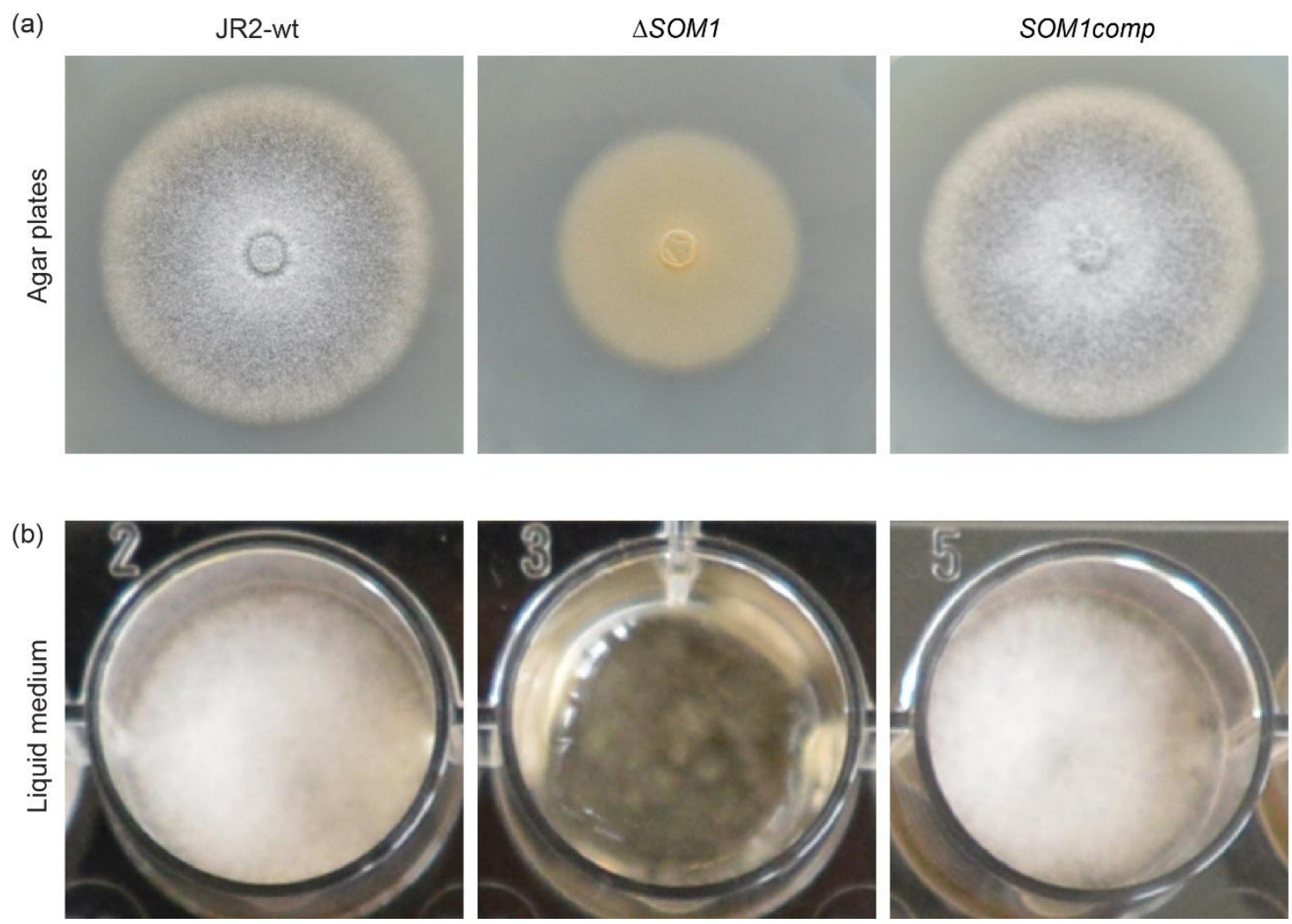

Figure 37. Som1 is necessary for aerial hyphae formation. (a) The same number of spores of the wild-type, SOM1 deletion, and complementation strains was dropped on agar plates and incubated in the dark at $25^{\circ} \mathrm{C}$ for seven days. (b) The indicated strains were grown in standing liquid SXM for seven days.

As mentioned in chapter 3.2.2.1, the biomass of the SOM1 deletion strain is higher than that of the wild-type and it grows slower on agar plates. Hyphal branching might be affected in the deletion of SOM1. Therefore, the branching of hyphae was further examined (Figure 38). As shown in Figure 38a, hyphal branches were observed more often in the SOM1 deletion strain than in the wild-type on agar 
plates. The formation of hyphal branches was further quantified by growing hyphae in devices with multiple channels (Stanley et al., 2014). The formation of hyphal branches was observed with a microscope. As seen in Figure 38b, the SOM1 deletion strain has on average eight branches whereas wild-type and complementation strains have only two branches in $750 \mu$ m hyphae measured from the hyphal tips (Figure 38b). Taken together, Som1 is required for hyphal branching.

(a)
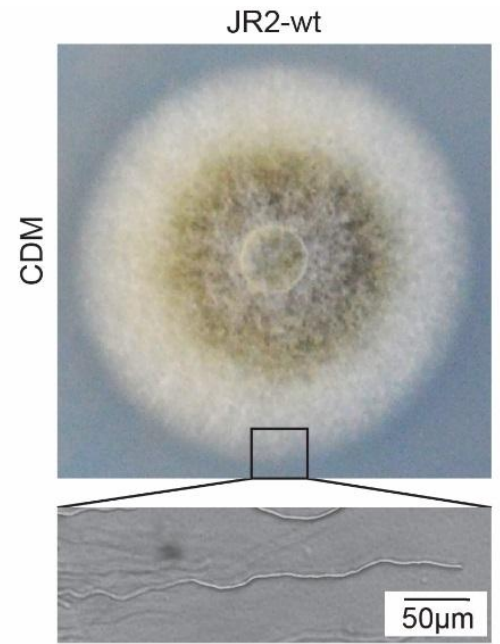

$\triangle S O M 1$

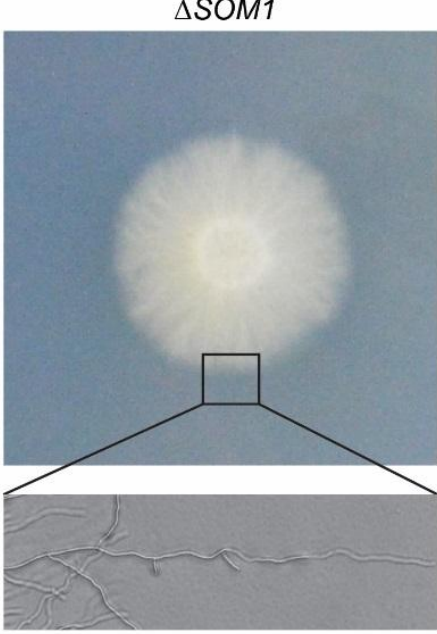

SOM1comp

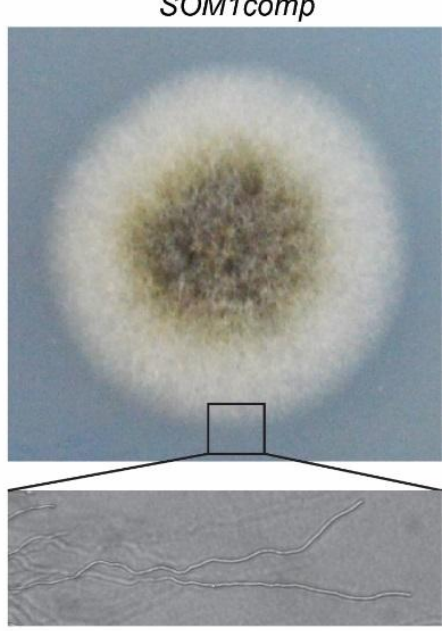

(b)

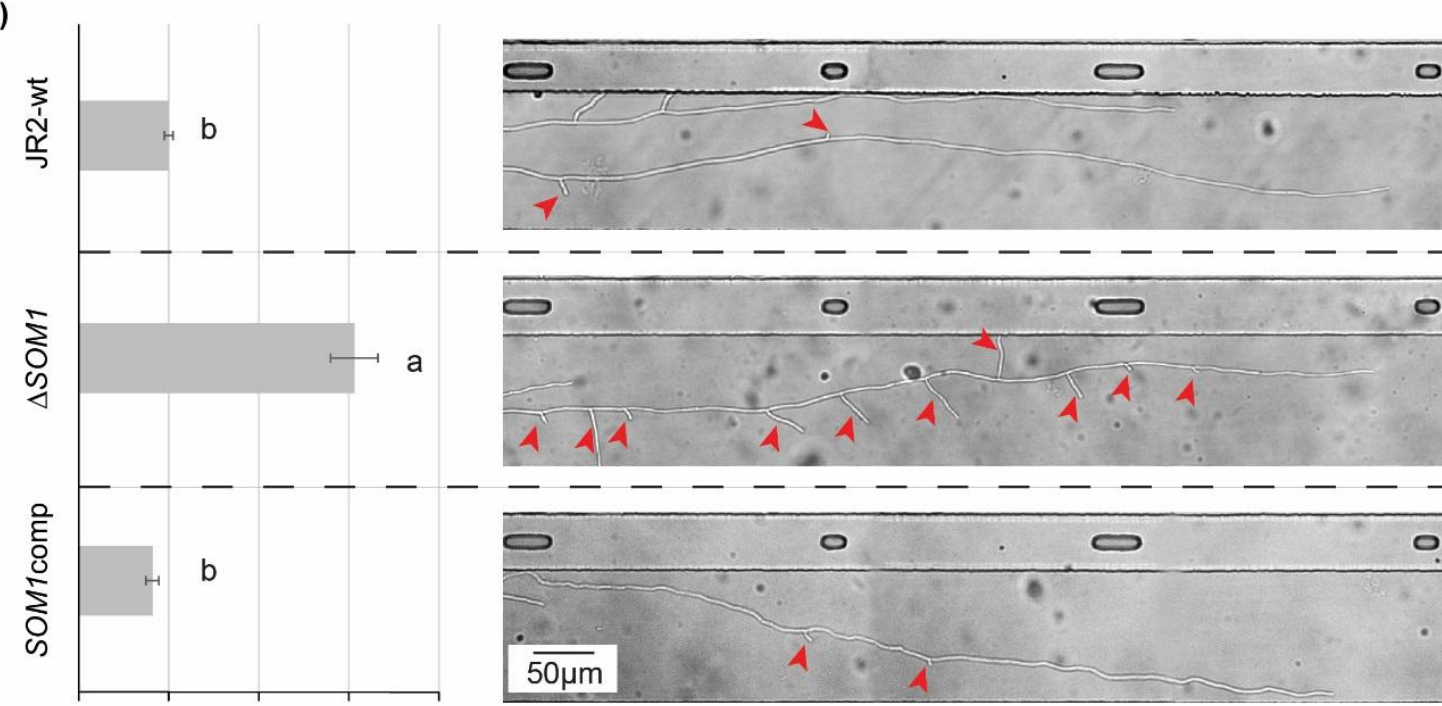

Number of branches relative to WT

Figure 38. Som 1 is required for aerial hyphae and hyphal branching of $V$. dahliae. (a) The same number of spores of JR2-wt, the SOM1 deletion, and complementation strains was dropped on CDM plates and grown in the dark at $25^{\circ} \mathrm{C}$ for ten days. The development of aerial hyphae and hyphal branching was observed on the surface of plates. (b) Quantification of hyphal branches. Single hyphae of indicated strains were grown in microchannels. Arrowheads indicates hyphal branches. The number of branches in $750 \mu \mathrm{m}$ hyphae was counted. The experiment was performed in triplicate. Average values and standard deviation are indicated. The letters $a$ and $b$ indicate the groups which are significantly different calculated by Tukey-Kramer multiple comparison procedures, $\alpha=0.01$. The arrowhead shows a hyphal branch. Scale bars are displayed. 
Cell morphology was also examined of the SOM1 deletion strain grown in liquid SXM for $24 \mathrm{~h}$. Cell walls and septa were stained with congo red and observed by a fluorescence microscope. The data in Figure 39a suggests that the SOM1 deletion strain has more septa than the wild-type in elongating hyphae. The distance between septa of the SOM1 deletion strain is approximately $50 \%$ shorter than that of the wild-type and complementation strains (Figure 39b).
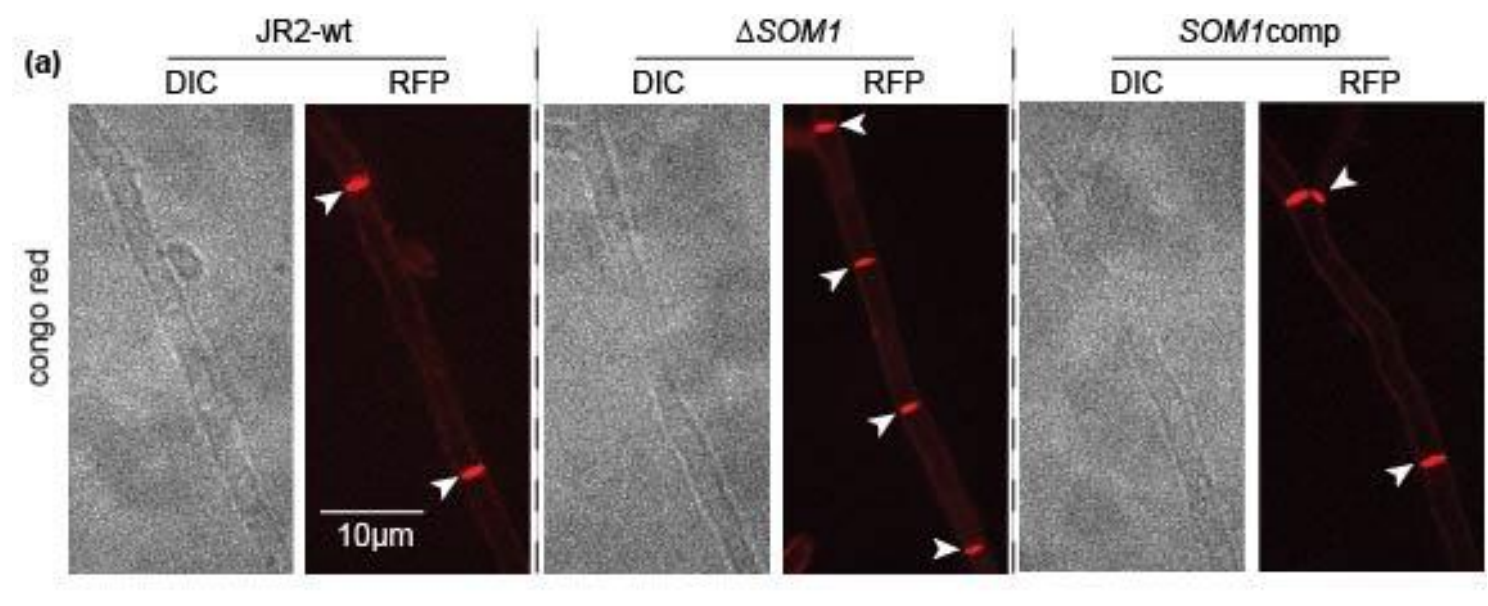

(b)

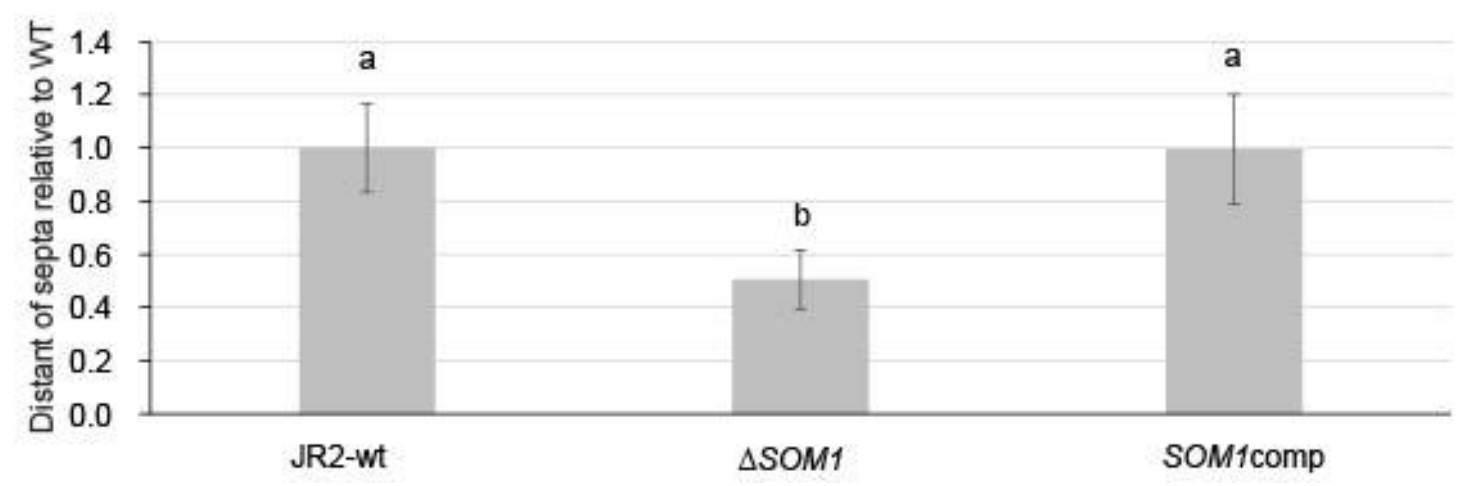

Figure 39. Som 1 is required for septa positioning in $\boldsymbol{V}$. dahliae. Spores of JR2-wt and SOM1 deletion and complementation strains were grown in SXM containing cefotaxime at $25^{\circ} \mathrm{C}$ for $24 \mathrm{~h}$. The pictures were taken by fluorescent microscopy with DIC and RFP filters. Septa and cell walls were stained with congo red. The distance between septa was measured (a) Septa positioning. White arrowheads indicate septa. (b) The diagram of septa distance in elongating hyphae. The experiments were performed in triplicate. One hundred cells were observed for each repetition. Mean values and standard deviation are represented. The letters $a$ and $b$ indicate groups which are significantly different as calculated by Tukey-Kramer multiple comparison procedures, $\alpha=0.01$. Scale bars are shown. 
Vacuoles in hyphae were dyed with FM4-64 and observed with a fluorescence microscope (Figure 40a). The diameter of vacuoles was also quantified as shown in Figure 40b. The vacuoles of SOM1 deletion strain were smaller than that of the wild-type strain. The diameter of vacuoles in the deletion strain was reduced by $60 \%$ compared to that of the wild-type strain. The vacuoles in wild-type are round and massive, whereas they are small and as dot-like structures in the SOM1 deletion strain (Figure 40b). In summary, Som1 is necessary for hyphal development including aerial hyphae formation, hyphal branching, septa positioning, and vacuole size.

(a)
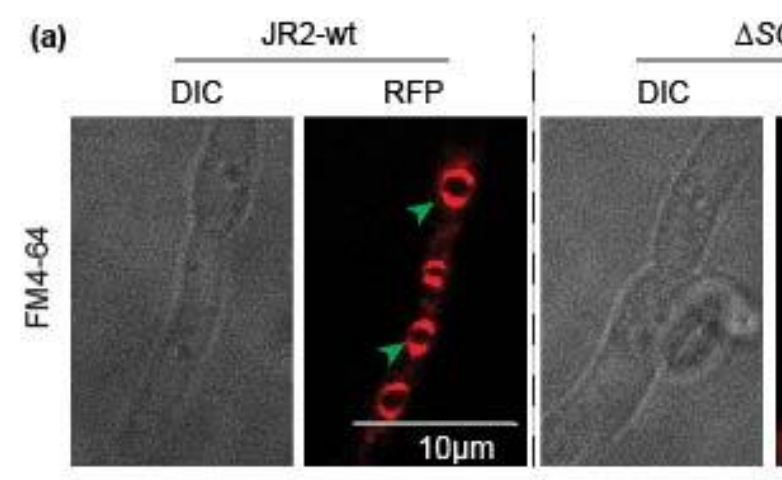

$\triangle S O M 1$
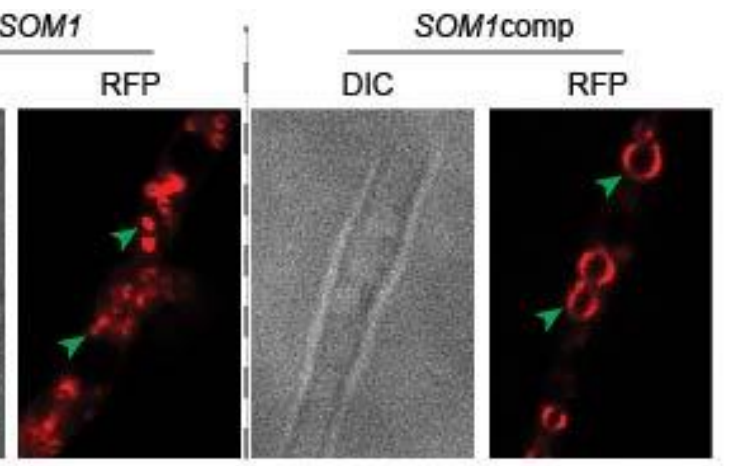

(b)

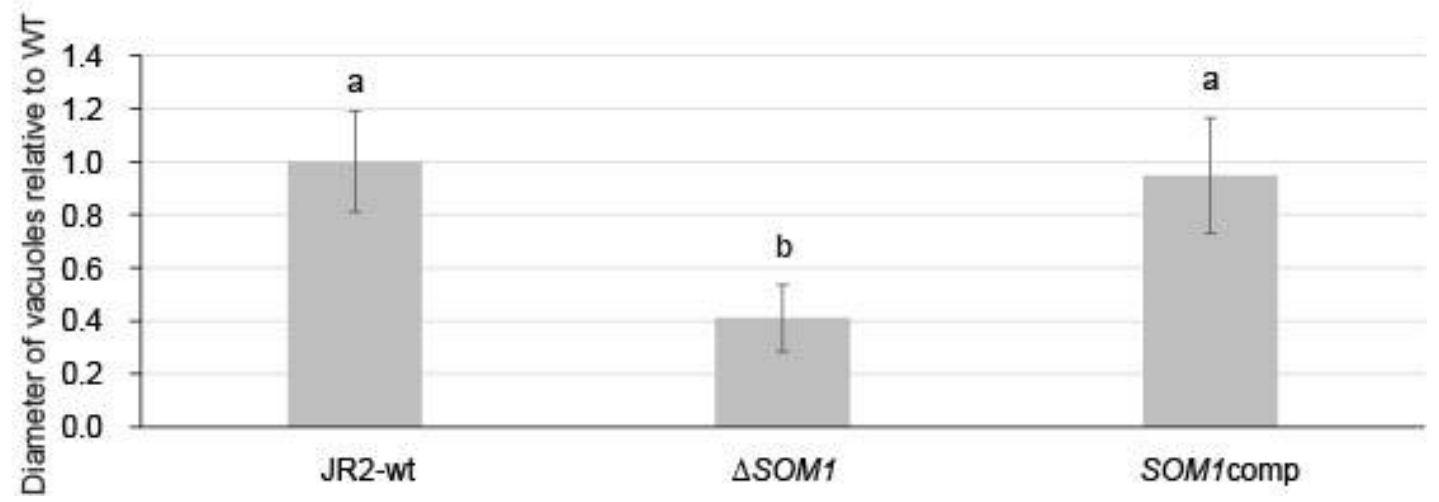

Figure 40. V. dahliae Som1 is essential for normal vacuole size. Spores of JR2-wt and SOM1 deletion and complementation strains were grown in SXM containing cefotaxime at $25^{\circ} \mathrm{C}$ for $24 \mathrm{~h}$. The pictures were taken by fluorescence microscopy with DIC and RFP filters. The vacuoles were dyed by FM4-64. The diameter of vacuoles was determined. (a) Stained vacuole in $V$. dahliae. The green arrowhead shows a vacuole. (b) Diagram of vacuole diameter. The experiments were performed in triplicates. One hundred cells were observed for each repetition. Mean values and standard deviation are shown. The letters $a$ and $b$ indicate groups which are significantly different as calculated by Tukey-Kramer multiple comparison procedures, $\alpha=0.01$. Scale bars are displayed. 


\subsubsection{Som1 and VTA3 regulate the expression of VTA genes and related adhesion, conidia and microsclerotia formation, and virulence genes}

Transcription factors for adhesion can reprogram adhesion in non-adhesive FLO8-defective S. cerevisiae strains. Therefore, Som1 might directly or indirectly regulate VTA genes. Additionally, the phenotypes are usually regulated by several genes. The expression of transcription factors for adhesion and genes which are involved in adhesion, conidiation, microsclerotia formation, oxidative stress response, and virulence was examined by real-time PCR in wild-type and the deletion strain.

\subsubsection{Som1 and Vta3 regulate the expression of VTA genes}

Verticillium transcription activator of adhesion (VTA) genes can rescue FLO8defected yeast strains as shown before (Tran et al., 2014). Som1 is a ortholog of Flo8 and therefore might regulate the expression of VTA genes in Verticillium. To test this hypothesis, the expression of six VTA genes was examined in SOM1 and VTA3 deletion strains (Figure 41). The expression of VTA1, VTA2, VTA3, VTA4, and VTA6 was significantly reduced in the deletion strain of SOM1. Interestingly, the expression of VTA2 and VTA3 is reduced by $90 \%$ and $94 \%$ in the SOM 1 deletion strain. In the VTA3 deletion strain, the expression of VTA1, VTA2, and VTA6 was significantly decreased, whereas the expression of VTA4 and VTA5 was unchanged (Figure 41). In summary, Som1 controls the expression of VTA3, and both, Som1 and Vta3, regulate the expression of VTA1, VTA2, and VTA6 genes.

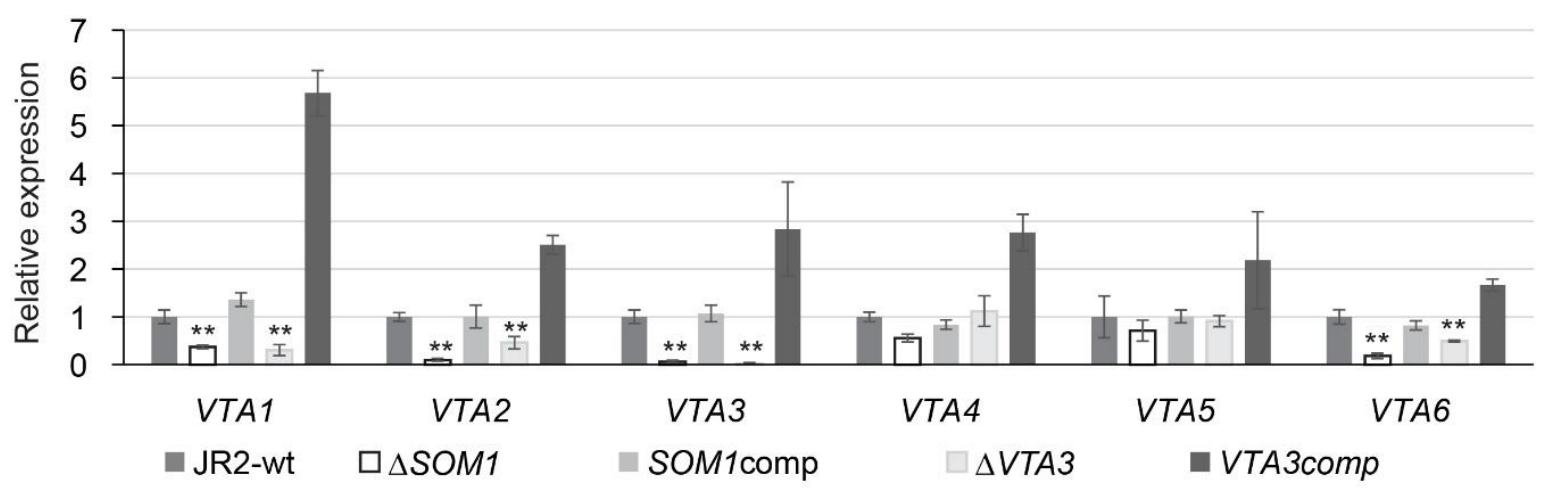

Figure 41. Som 1 and Vta3 control the expression of VTA genes. The RNAs were purified from three days old mycelia grown in $50 \mathrm{ml}$ liquid SXM. The same amount of RNA was used for cDNA synthesis. The expression of putative target genes was normalized to a housekeeping gene (GAPDH) and the wild-type strain. The mean values and standard deviations of four repetitions are represented. The asterisk $\left(^{* *}\right)$ indicates a significant decrease when compared to the JR2-wt as calculated by Student's t-test, $p<0.01$. 


\subsubsection{Som1 control expression of genes involved in adhesion}

Putative adhesion proteins are usually localised on the cell surface (Sundstrom, 2002). The deletion strain of SOM1 grown in liquid MM defects in adhesion on abiotic surfaces (Figure 27). Som1 might control the expression of some adhesive factors. Therefore, cell lysate protein of JR2-wt and SOM1 deletion and complementation strains, which were grown in liquid MM, was isolated and further analysed in 12\% SDS gel. Each band was separately digested with trypsin in gels. Peptides from each sample were independently evaluated by mass spectrometry and examined by the MaxQuant program. Proteins, which is significantly different in abundance, are shown in Table 4. The data show that twenty proteins were not found or rarely detected in the SOM1 deletion strain, whereas they are abundant in the wild-type and complementation strains (Table 4). 
Table 4. List of abundant proteins significantly changed in the SOM1 deletion strain

\begin{tabular}{|c|c|c|c|c|c|c|c|c|}
\hline 1 & 2 & 3 & 4 & 5 & 6 & 7 & 8 & 9 \\
\hline G2WW49 & $\begin{array}{l}\text { Fatty acid synthase S- } \\
\text { acetyltransferase }\end{array}$ & 24.7 & 24.5 & 1.0 & 26.3 & 86.3 & 0.3 & 303.7 \\
\hline G2XHAO & Serine 3-dehydrogenase & 24.2 & 25.9 & 1.0 & 24.9 & 120.7 & 0.5 & 63.5 \\
\hline G2XG83 & YcaC & 22.7 & 22.7 & 1.0 & 24.1 & 20.7 & 0.0 & 29.5 \\
\hline G2XK31 & Sulfite oxidase & 22.2 & 22.6 & 1.0 & 23.5 & 31.0 & 0.2 & 47.7 \\
\hline G2XH97 & Putative uncharacterized protein & 20.8 & 22.9 & 1.0 & 21.3 & 35.0 & 0.0 & 12.8 \\
\hline G2X049 & NADPH dehydrogenase & 20.5 & 21.3 & 1.0 & 21.6 & 8.3 & 0.0 & 10.5 \\
\hline G2XH96 & Putative uncharacterized protein & 20.4 & 22.4 & 1.0 & 20.8 & 28.7 & 0.0 & 8.0 \\
\hline G2XJ01 & Reticulon-4-interacting protein & 20.2 & 20.5 & 1.0 & 21.5 & 7.7 & 0.0 & 13.8 \\
\hline G2WS45 & Nitrate reductase & 19.2 & 20.4 & 1.0 & 20.0 & 9.3 & 0.2 & 4.7 \\
\hline G2WZ45 & Putative uncharacterized protein & 18.9 & 19.9 & 1.0 & 19.9 & 6.3 & 0.0 & 5.5 \\
\hline G2XAR8 & $\begin{array}{l}\mathrm{HpcH} / \mathrm{Hpal} \text { aldolase/citrate lyase } \\
\text { family protein }\end{array}$ & 18.0 & 22.0 & 4.0 & 21.9 & 15.7 & 0.5 & 12.3 \\
\hline G2X086 & $\begin{array}{l}\text { Tetrahydroxynaphthalene } \\
\text { reductase }\end{array}$ & 17.7 & 13.5 & 1.0 & 21.4 & 6.3 & 0.0 & 19.2 \\
\hline G2X5M1 & $\begin{array}{l}\text { Short-chain } \\
\text { dehydrogenase/reductase SDR }\end{array}$ & 16.0 & 19.6 & 3.9 & 20.1 & 4.0 & 1.0 & 6.3 \\
\hline G2XFZ6 & Acyl-CoA desaturase & 14.4 & 19.7 & 3.9 & 17.5 & 3.3 & 0.3 & 9.7 \\
\hline $\mathrm{G} 2 \mathrm{X} 1 \mathrm{CO}$ & Cap20 protein & 13.3 & 19.7 & 7.1 & 20.7 & 4.7 & 1.8 & 8.0 \\
\hline G2X9D9 & Ribitol kinase & 13.3 & 19.5 & 6.8 & 20.3 & 3.7 & 0.8 & 5.5 \\
\hline G2X8Q8 & SnodProt1 & 11.5 & 20.5 & 9.7 & 21.5 & 4.0 & 1.3 & 6.0 \\
\hline G2XGN9 & ThiJ/Pfpl family protein & 11.4 & 20.5 & 6.9 & 17.3 & 8.7 & 1.7 & 5.5 \\
\hline G2X061 & Alpha/beta hydrolase & 10.5 & 19.9 & 7.1 & 16.5 & 6.0 & 2.5 & 5.5 \\
\hline G2X1B6 & $\begin{array}{l}\text { Ribonucleoside-diphosphate } \\
\text { reductase }\end{array}$ & 10.4 & 20.1 & 9.9 & 20.3 & 7.3 & 1.3 & 9.5 \\
\hline
\end{tabular}

The same amount of cell lysate was run in 12\% SDS-PAGE and digested with trypsin. The protein list was identified by LC-MS/MS and MaxQuant analysis and further processed with Perseus. Contaminants, and log2 (fold change) lower than 10 were removed from the final analysis. Columns: 1-accession number, 2-cellular function, 3-log2 (fold change), 4mean of log2 (LFQ intensity of JR2-wt), 5-mean of log2 (LFQ intensity of $\triangle S O M 1$ ), 6-mean of log2 (LFQ intensity of SOM1comp), 7-mean of MS/MS count of JR2-wt, 8-mean MS/MS count of $\triangle S O M 1,9-$ mean of MS/MS count of SOM1comp. The table summarized the results from three independent biological repetitions and three technical replicates. 
The twenty proteins identified were grouped into three clusters according to their function: cell rescue, defence and virulence (10\%), metabolism (70\%), and unknown function (20\%). The clusters are shown in Figure 42 . The cluster cell rescue, defence and virulence included the proteins Cap20 and Snod1.

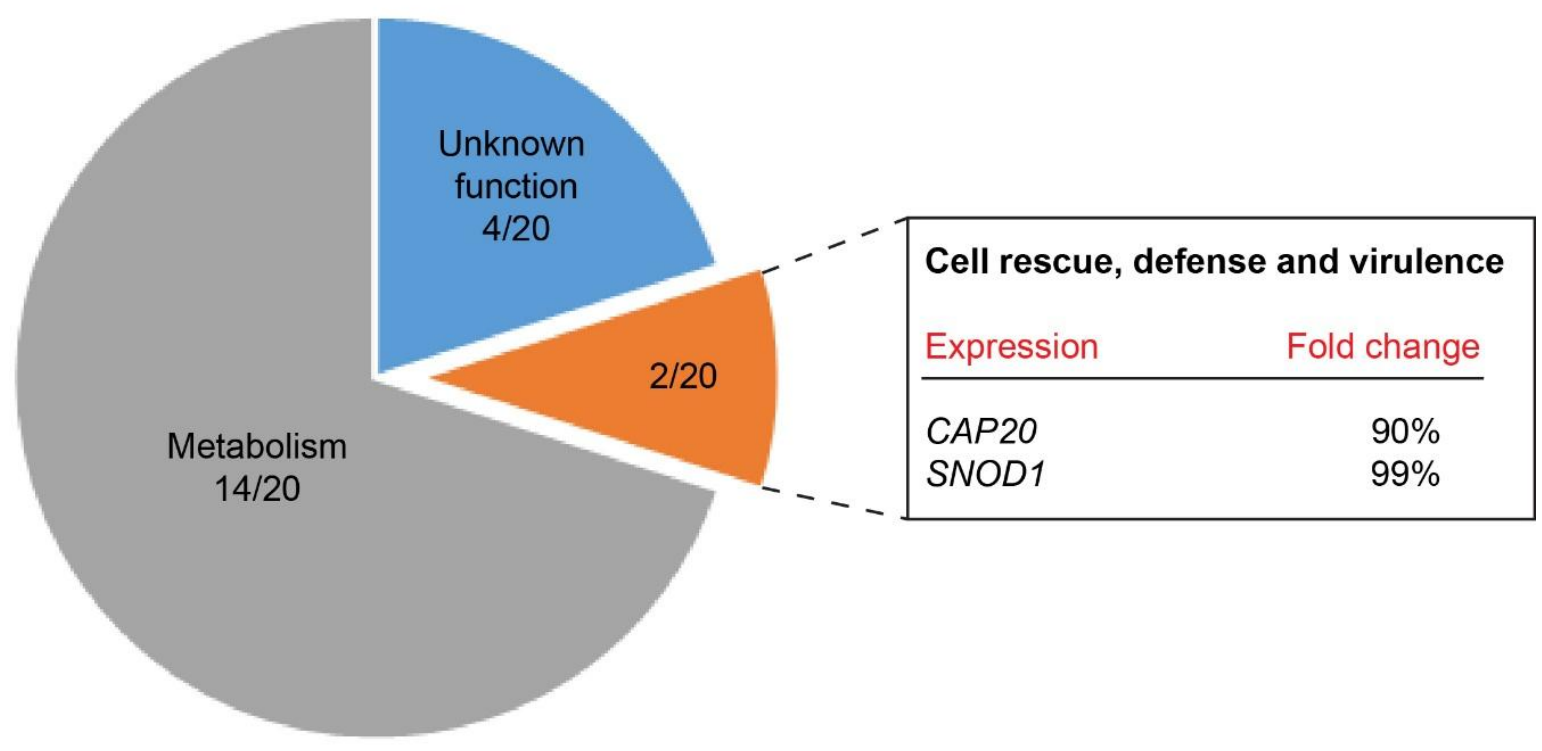

Figure 42. Functional categorisation analyses of twenty proteins significantly downregulated in the SOM1 deletion strain. The functional categorisation of proteins was done by using the Funcat version 2.0 (Ruepp et al., 2004). Main groups of protein functions are indicated. The expression of genes was confirmed by real-time PCR. The expression was normalized to a housekeeping gene (GAPDH) and the wild-type. The fold changes are shown.

The expression of genes required for adhesion was investigated by real-time PCR as seen in Figure 43. The expression of FAS1, MAD1, MAD2, CAP20, SNOD1, and PLS1 was significantly reduced in the SOM1 deletion strain by $80 \%, 94 \%, 90 \%$, $84 \%, 87 \%$, and $98 \%$, respectively (Figure 43 ). Taken together, Som 1 promotes adherence via controlling the expression of putative target genes for adhesion. 


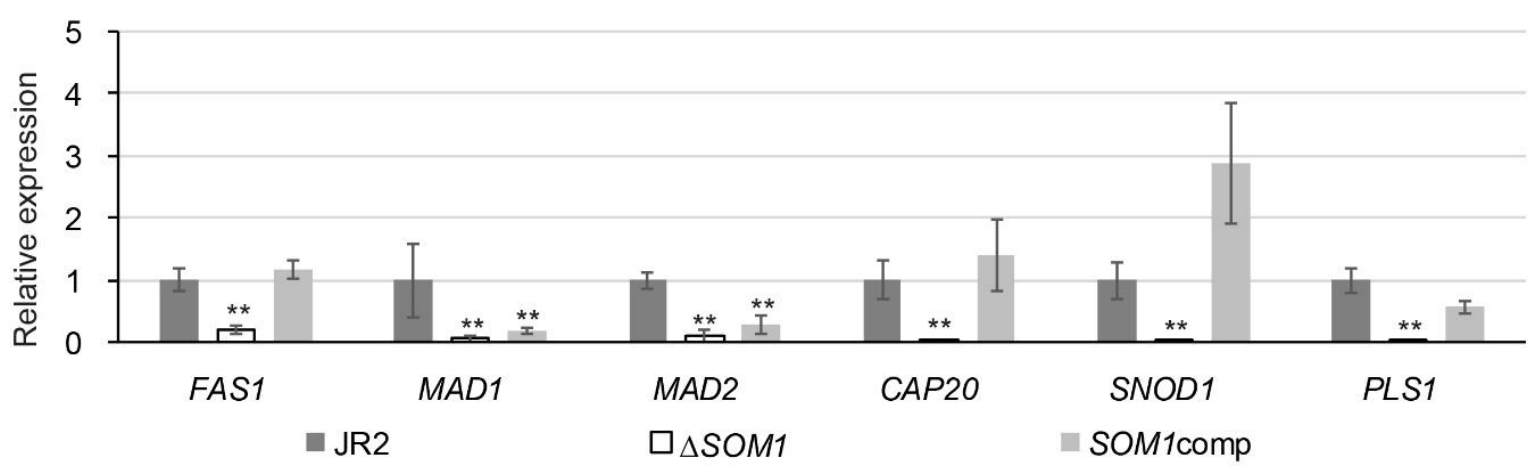

Figure 43. Som1 controls the expression of putative adhesion target genes. The RNAs have been purified from three days old mycelia grown in $50 \mathrm{ml} \mathrm{SXM}$. The same amount of RNA was used for cDNA synthesis. The expression of putative target genes was normalized to a housekeeping gene (GAPDH) and the wild-type strain. The mean values and standard deviations of four repetitions are represented. The asterisk $\left({ }^{* *}\right)$ indicates a significant decrease when compared to the JR2-wt as calculated by Student's t-test, $p<0.01$.

\subsubsection{Som1 and Vta3 control expression of genes involved in conidia and microsclerotia formation, oxidative stress response and virulence}

Som1 and Vta3 are required for conidia and microsclerotia formation (Figure 32, 33, 34), and virulence (Figure 28, 29, 30, 31), antagonised the oxidative stress response (Figure 35) and adhesion (Figure 26, 27), therefore, the expression of genes involved in these processes was investigated. The results are represented in Figure 44.

Five genes involved in conidia formation were investigated, namely VTA2, $C O N-6, C O N-8, C O N-10$, and $A B A 1$. The expression of genes involved in conidia formation was significantly reduced in both deletion strains. The transcript levels of VTA2, CON-6, CON-8, CON-10, and ABA1 in the SOM1 deletion strain were reduced by $90 \%, 64 \%, 70 \%, 92 \%$, and $98 \%$ compared to the wild-type, respectively (Figure 41, 44a). Reduced transcript levels were also observed for CON-8 and $A B A 1$ in the VTA3 deletion strain corresponding to $70 \%$ and $57 \%$ decrease, respectively (Figure 44b).

Significant decrease in the expression of $V D H 1$, required for microsclerotia formation, was also observed in SOM1 and VTA3 deletion strains when compared to the wild-type corresponding to $95 \%$ decrease. The expression of genes involved in oxidative stress response was also examined. While the expression of genes VEL1, CPX2 and SOD3 was significantly decreased in the SOM1 deletion strain by $93 \%, 96 \%$ and $94 \%$, respectively, the expression of INO1 in the VTA3 deletion strain was significantly enhanced. 
Furthermore, the expression of genes which are putatively involved in virulence was significantly lowered in the deletion strains of SOM1 and VTA3. The expression of VTA2, CAP20, SNOD1, NLP2, and PRY1 was significantly reduced in the SOM1 deletion strain by $90 \%, 90 \%, 99 \%, 71 \%$, and $99 \%$, respectively (Figure 41, 43, 44a). The expression of VTA2, CAP20, NLP2, and PRY1 was significantly decreased in VTA3 deletion strain by $54 \%, 60 \%, 67 \%$, and $83 \%$ (Figure 41, 44b), whereas expression of SNOD1 is unchanged. Taken together, Som1 and Vta3 control the expression of genes which might be involved in the control of conidia and microsclerotia formation, oxidative stress response, and virulence.

(a)

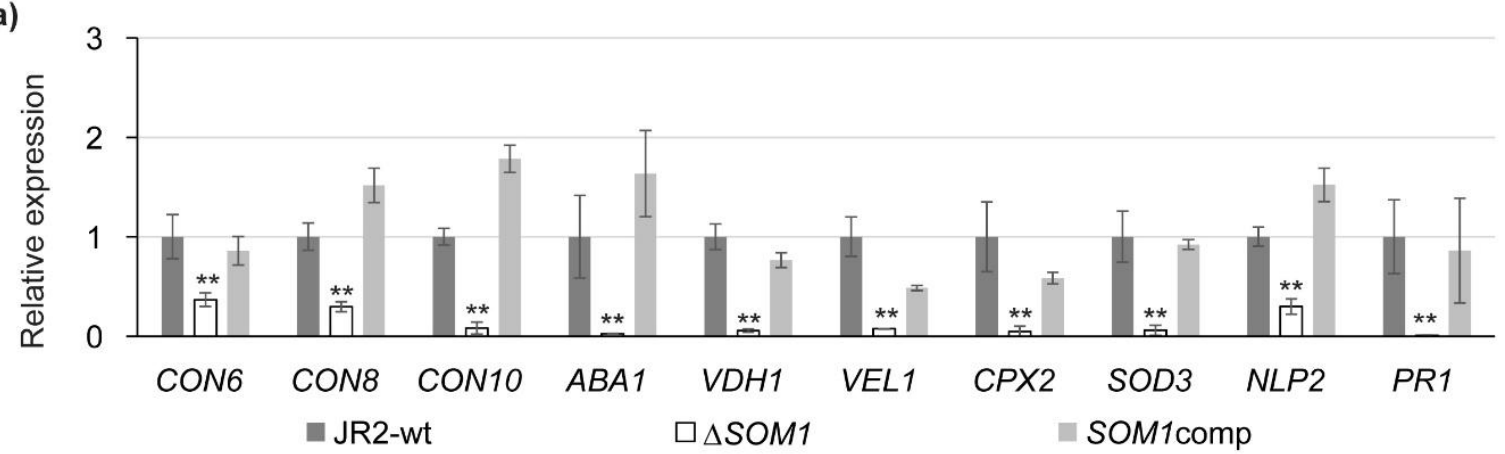

(b)

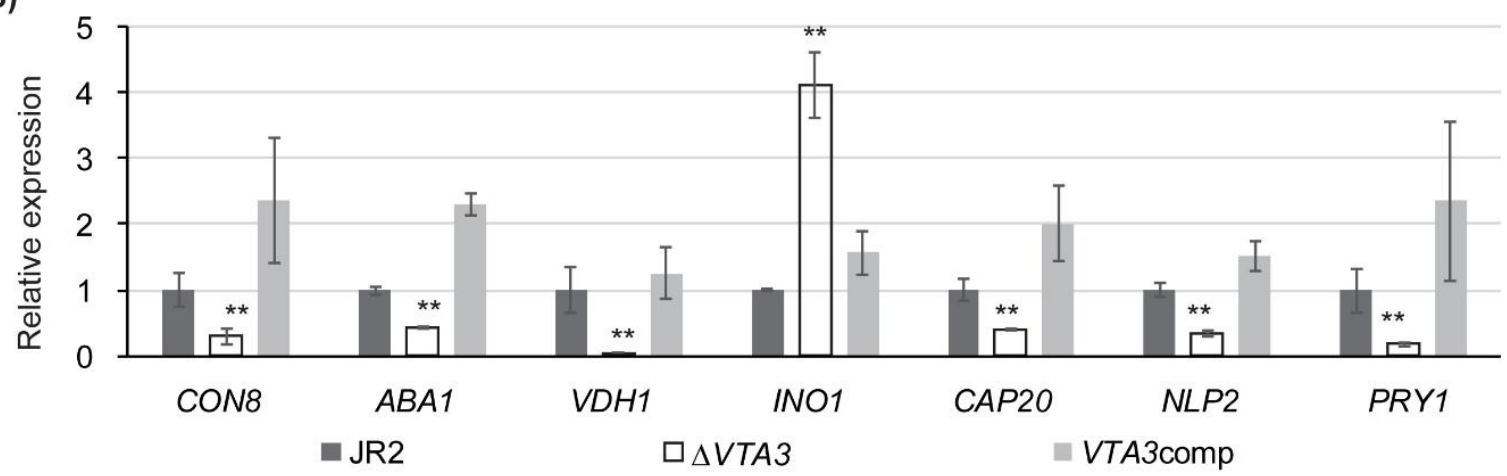

Figure 44. Som 1 and Vta3 control the expression of putative target genes of conidia and microsclerotia formation, oxidative stress response, and virulence. The RNAs were purified from three days old mycelia grown in $50 \mathrm{ml}$ SXM. The same amount of RNA was used for cDNA synthesis. The expression of putative target genes was normalized to a housekeeping gene (GAPDH) and the wild-type strain. The mean values and standard deviations of 4 repetitions are represented. (a) Som 1 regulates the expression of putative target genes for conidiation, microsclerotia formation, oxidative stress response, and virulence genes. (b) Vta3 controls the expression of assumed target genes for conidia and microsclerotia formation, stress response, and virulence. The asterisk $\left({ }^{* *}\right)$ indicates a significant decrease when compared to the JR2-wt as calculated by Student's t-test, $p<0.01$. 


\subsubsection{Som1 interacts with protein Ptab while Vta3 interacts with the transcriptional co-repressor Cyc8}

Often proteins interact with other protein to fulfil their functions (Phizicky \& Fields, 1995). The interaction partner of Som1 and Vta3 were investigated by pull- down assays and the results are displayed in Table 5 and Table 6. The Table 5 shows seven proteins which are only found in the Som1:GFP sample (Table 5). In which, Ptab was shown with high log2 (LFQ intensity) and high MS/MS count and appeared in all replication. Ptab is a homolog of Mfg1 in S. cerevisiae which is reported to be an interaction partner of Flo8.

Table 5. Som1 putative interaction partners identified by GFP-trap enrichment

\begin{tabular}{|c|l|c|c|c|c|}
\hline \multicolumn{1}{|c|}{$\mathbf{1}$} & \multicolumn{1}{|c|}{$\mathbf{3}$} & $\mathbf{4}$ & $\mathbf{5}$ & $\mathbf{6}$ \\
\hline G2X5W3 & Som1 protein & 17.2 & 18.2 & 6.4 & $6 / 6$ \\
\hline G2X127 & Ptab protein & 12.1 & 7.6 & 2.8 & $6 / 6$ \\
\hline G2X461 & Tyrosinase & 7.2 & 1.9 & 1.4 & $4 / 6$ \\
\hline G2WW49 & Fatty acid synthase S- & 7.2 & 20.9 & 13.9 & $3 / 6$ \\
\hline G2XK31 & Sulfite oxidase & 7.0 & 5.0 & 3.2 & $4 / 6$ \\
\hline G2WS88 & Glutathione S-transferase Gst3 & 6.1 & 3.1 & 2.4 & $3 / 6$ \\
\hline G2X411 & 26S protease regulatory subunit 4 & 4.7 & 1.1 & 0.9 & $3 / 6$ \\
\hline
\end{tabular}

Proteins, identified by LC-MS/MS after enrichment of SOM1:GFP by GFP-trap, were examined. Contaminants and proteins shown to interact with free GFP were filtered out. Proteins in this list were only found in the SOM1:GFP at least five times of log2 (intensity) higher than 11. Columns: 1-protein IDs, 2-cellular function, 3-mean of log2 (LFQ intensity), 4mean of MS/MS count, 5-min unique peptides, 6-appearance times. The table summarized the results from three independent biological pull downs and two technical replicates.

Table 6 displays five proteins which are only detected in the Vta3:GFP sample (Table 6). The Cyc8 protein was identified in the list of protein enriched by Vta3::GFP. Transcriptional co-repressor Cyc8 is a ortholog of Ssn6 in S. cerevisiae which was shown to be a interaction partner of Rfx1 and of SsnF which had been described in A. fumigatus (Huang et al., 1998; Johnk et al., 2016). Taken together, Som1 might interact with six proteins including Ptab while Vta3 might physically bind to four proteins including Ssn6. 
Table 6. Vta3 putative interaction partners identified by GFP-trap enrichment

\begin{tabular}{|l|l|c|c|c|c|}
\hline \multicolumn{1}{|c|}{$\mathbf{1}$} & \multicolumn{1}{c|}{$\mathbf{2}$} & $\mathbf{3}$ & $\mathbf{4}$ & $\mathbf{5}$ & $\mathbf{6}$ \\
\hline G2WUC4 & Sak1 & 15.4 & 26.5 & 16.0 & $9 / 9$ \\
\hline G2WR58 & Inositol-3-phosphate synthase & 6.6 & 5.0 & 8.0 & $3 / 9$ \\
\hline G2XH18 & $\begin{array}{l}\text { H/ACA ribonucleoprotein complex } \\
\text { subunit 1 }\end{array}$ & 5.1 & 2.4 & 2.0 & $3 / 9$ \\
\hline G2WXX5 & Sulfate adenylyltransferase & 5.1 & 1.8 & 4.0 & $4 / 9$ \\
\hline G2XAE9 & Transcriptional co-repressor Cyc8 & 4.1 & 1.3 & 5.0 & $3 / 9$ \\
\hline
\end{tabular}

Proteins, identified by LC-MS/MS analyses after enrichment of VTA3:GFP by GFP-trap, were investigated. Contaminants and proteins shown to interact with free GFP were filtered out. Proteins in this list consist of only proteins found in the VTA3:GFP at least three times of log2 (intensity) higher than 11. Columns: 1-protein IDs, 2-cellular function, 3-mean of log2 (LFQ intensity), 4-mean of MS/MS count, 5-mean unique peptides, 6-appearance times. The table summarized the results from three independent biological pull downs and three technical replicates.

\subsection{A. fumigatus SomA can rescue the deletion of SOM1 in $V$. dahliae}

Alignment of the plant pathogen $V$. dahliae SOM1 sequence and the opportunistic pathogen $A$. fumigatus AfSOMA shows $31 \%$ similarity. These proteins fulfill similar functions in different pathogenic fungi such as in adhesion, conidiation, aerial hyphae formation, and virulence (Lin et al., 2015). GFP trap results indicate that Som1 physically interacts with Ptab (Table 5) as AfSomA in the human pathogenic fungus (Lin et al., 2015). It was examined whether Som1 fulfills similar functions as AfSomA in A. fumigatus and/or vice versa. The ORF of AfSOMA under the control of the gdpA promoter was transformed into the SOM1 deletion strain (Table 3). The results of the phenotype analysis indicate that AfSom 1 can rescue growth defects of the SOM1 deletion strain. The strain expressing AfSOMA grows faster than the deletion strain, but it still grows slower than the wild-type strain. Its growth can reach approximately $77 \%$ of the wild-type strain (Figure 45 ). Additionally, AfSom 1 can rescue the formation of aerial hyphae of deletion strain. The colony of the strain expressing of AfSOMA appears with the black ring and has aerial hyphae. Therefore, it might restore conidia and microsclerotia formation. 
(a)

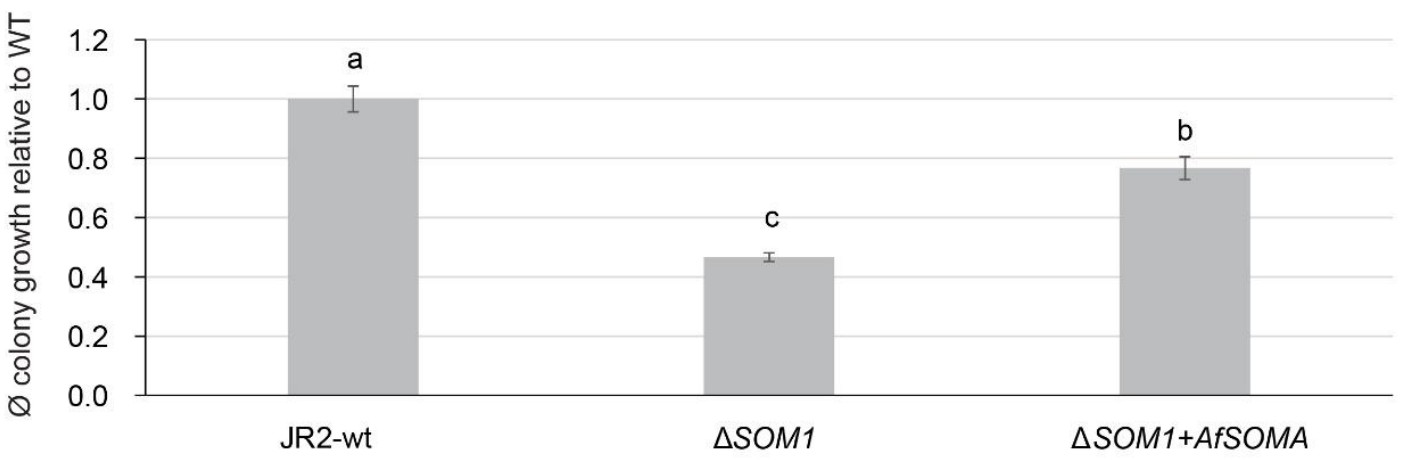

(b)

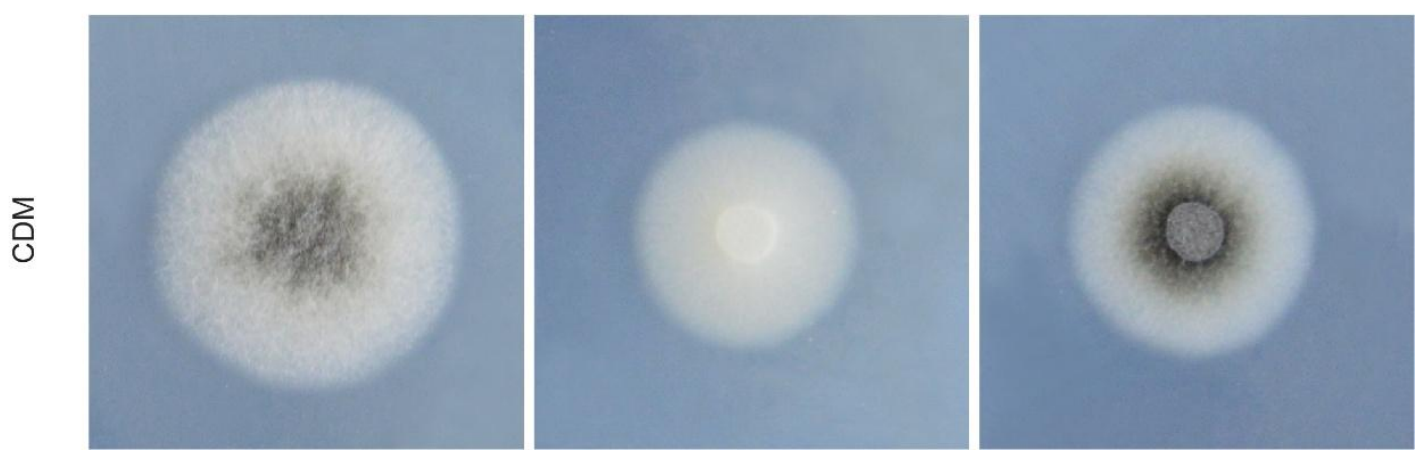

Figure 45. AfSomA can partly rescue the growth of the SOM1 deletion strain. The same spore numbers of the wild-type, SOM1 deletion, AfSOMA complementation strains were dropped on agar plates and incubated in the dark at $25^{\circ} \mathrm{C}$ for 11 days. (a) The diagram of the diameter of the colony at 11 dpi. (b) The colony growth on CDM plate at $11 \mathrm{dpi}$. The experiments were performed in triplicate. The mean values and standard deviations are shown. The letters a-c indicate groups which are significantly different as calculated by Tukey-Kramer multiple comparison procedures, $\alpha=0.01$.

The data of conidia and microsclerotia formation test indicate that AfSomA can rescue both, conidia and microsclerotia formation. Conidia formation of the wild-type and strain expression of AfSOMA are indistinguishable, whereas that of deletion strain is significantly reduced by $92 \%$ (Figure $46 \mathrm{a}$ ). The formation of microsclerotia of a strain expressing of AfSOM1 is not different than that of the wild-type strain. Microsclerotia are easy detected in strain expression of AfSomA and the wild-type, whereas they are unable to find in deletion of SOM1 (Figure 46b). In summary, Som 1 and AfSomA fulfill similar functions in the human pathogen A. fumigatus as in the plant pathogen $V$. dahliae. 
(a)

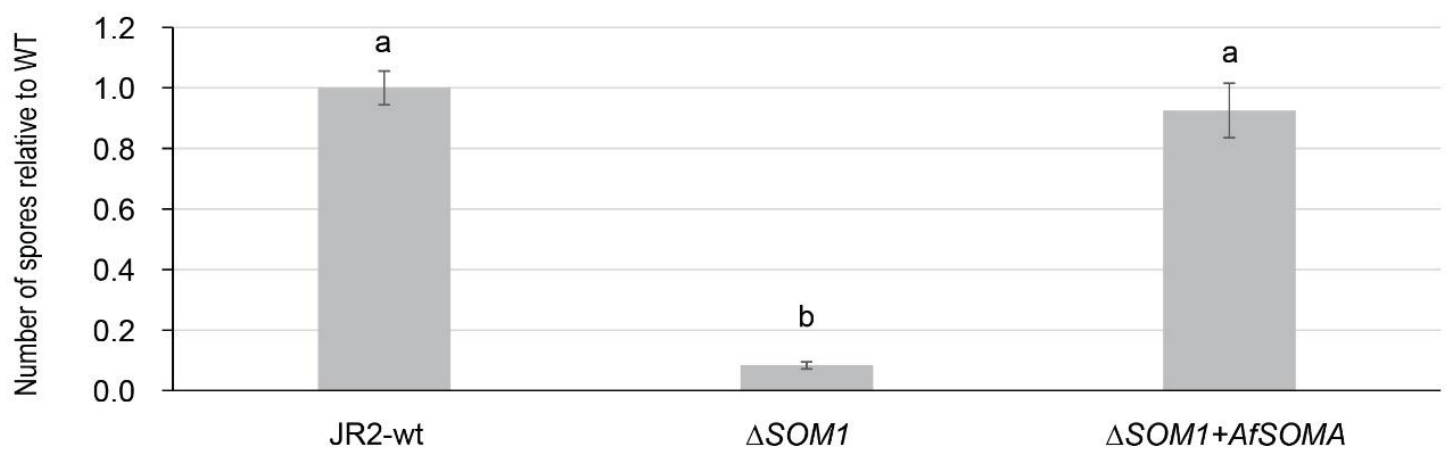

(b)
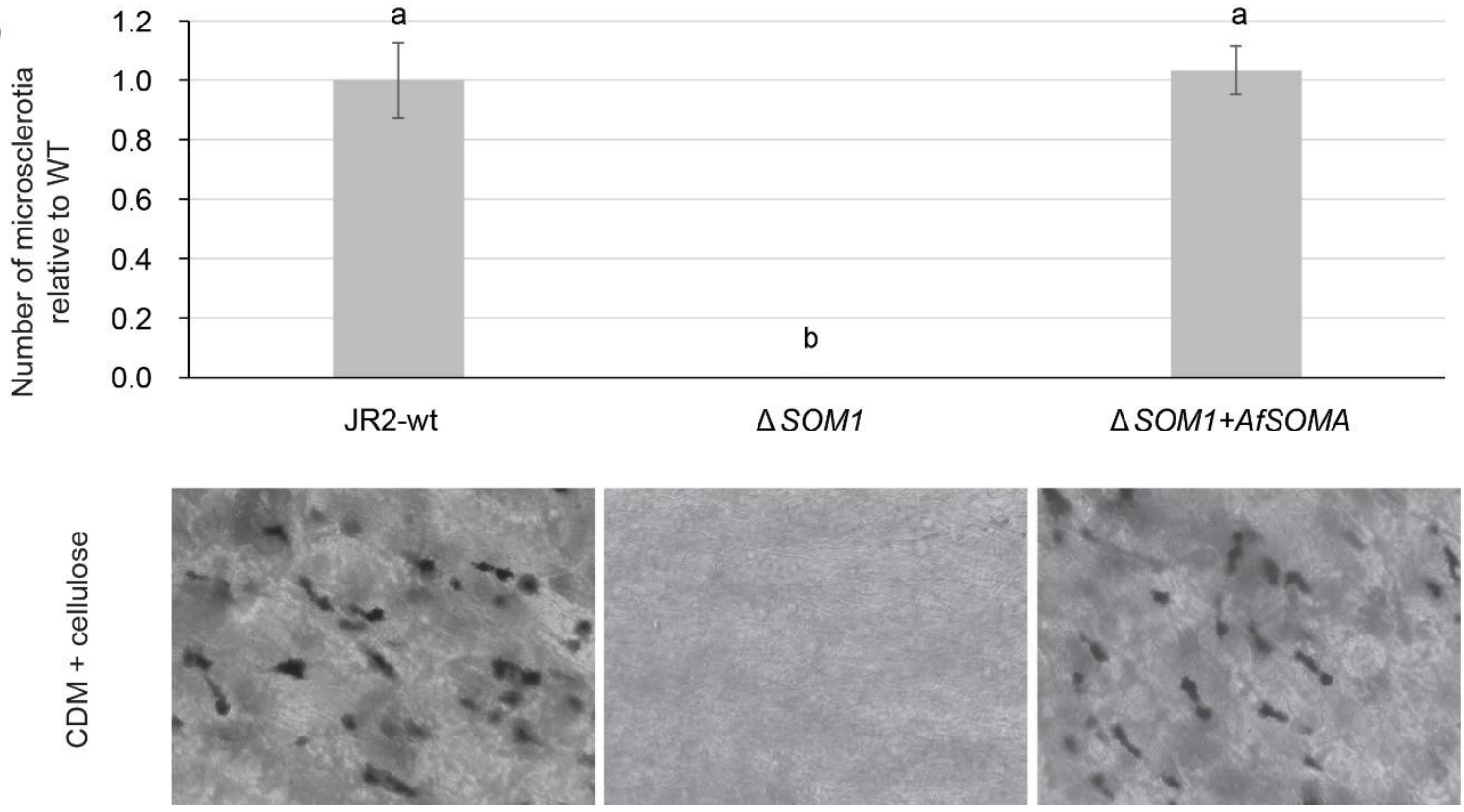

Figure 46. AfSomA can restore the conidia and microsclerotia formation in the SOM1 deletion strain of $\mathbf{V}$. dahliae. (a) Conidia formation was examined in $50 \mathrm{mI} \mathrm{SXM}$ on a shaker at $25^{\circ} \mathrm{C}$. The number of spores was counted at seven days post inoculation. (b) The same number of spores of indicated strains was dropped on CDM plates and incubated in the dark at $25^{\circ} \mathrm{C}$. Microsclerotia formation was tested on agar plates and incubated in the dark. The number of microsclerotia was counted after seven days. The experiments were performed in triplicates. The mean values and standard deviations are shown. The letters $a$ and $b$ indicate groups which are significantly different as calculated by Tukey-Kramer multiple comparison procedures, $\alpha=0.01$. 


\section{Discussion}

\subsection{The transcription factors Som1 and Vta3 support in adhesion of S. cerevisiae}

Adhesion is essential for fungal plant and human infections (Yan et al., 2011; Lin et al., 2015). This is also the case for the plant pathogenic fungus Verticillium for which it was shown that Verticillium transcriptional regulator of adhesion 2 (Vta2) is important for both, the infection and fungal development (Tran et al., 2014). In this study, we investigated the two proteins Som1 and Vta3, which are involved in fungal differentiation processes and infection and can restore the adhesion process in nonadherent $S$. cerevisiae. Both proteins can activate the expression of flocculation genes in S. cerevisiae using two distinct mechanisms.

\subsubsection{Som1 presumably binds to promoter regions of flocculation genes in $S$. cerevisiae for activation}

Som1, a homolog of Flo8, contains the LisH domain which is highly conserved. Low expression of Som 1 can rescue adhesion of non-adhesive FLO8 defective S. cerevisiae. Som1 might directly bind to promoter regions of flocculation genes for activation and interruption of the function of the inhibitor Sfl1 (Figure 47). It might act as its homolog in A. fumigatus and S. cerevisiae (Rupp et al., 1999; Lin et al., 2015). In S. cerevisiae, Flo8 regulates the expression of flocculation gene including FLO1 and FLO11 (Kobayashi et al., 1996; Fichtner et al., 2007). Flo1 is required for both, cell-cell and cell-substrate adhesion, whereas Flo11 is essential for adhesion of several layers of cells on agar plates (Fichtner et al., 2007). Flo11 is regulated by the cAMP/PKA and MAPK pathways. The MAPK pathway controls the expression of FLO11 via transcription factors Ste12 and Tec1, whereas the cAMP/PKA pathway regulates FLO11 through the activator Flo11 and the inhibitor Sfl1. Flo8 and Sfl1 directly bind and have an overlapping binding side on the promoter region of FLO11. Flo8, Ste12, and Tec1 directly bind to the promoter region of FLO11 for controlling the expression (Octavio et al., 2009). Disruption of FLO8, STE12, or TEC1 causes a block of FLO11 expression and leads to defects in adhesion (Rupp, Steffen et al., 1999; Fichtner et al., 2007). Expression of AfSOMA, a homolog of FLO8 in A. fumigatus, can activate FLO1 and FLO11 genes in FLO8-defective yeast. 
AfSomA of $A$. fumigatus can act similarly to yeast Flo8 in the promoter region of FLO11 (Lin et al., 2015).

Som1, under the control of the GAL promoter, was not identified in yeast screen experiments (Tran et al., 2014). In our study, we found the reason why Som1 was not distinguished in adhesive screen experiments. The high expression of SOM1 cannot reprogram adhesion and activate the expression of FLO1 and FLO11. Additionally, the data show that the expression of STE12 is significantly down-regulated in the yeast strain with strong expression of SOM1. The overexpression of SOM1 inhibits the expression of STE12 and might cause an inactivation of FLO11 expression. Som1 might bind to the promoter region of flocculation genes but lacks the function of STE12 and therefore it cannot activate the expression of FLO11. Flo8 and Ste12 are essential for expression of FLO11 (Rupp et al., 1999). Lacking either Flo8 or Ste12 leads to defective adhesion in S. cerevisiae (Rupp et al., 1999; Kim et al., 2014). Taken together, the low expression of SOM1 can rescue adhesion in non-adhesion yeast. It might directly bind to the promoter of FLO11 for activating. The binding sites of Flo8 and Sfl1 in the promoter of FLO11 are overlapping (Octavio et al., 2009). Sfl1 is essential for the repression of flocculation genes (Conlan \& Tzamarias, 2001; Ansanay Galeote et al., 2007).

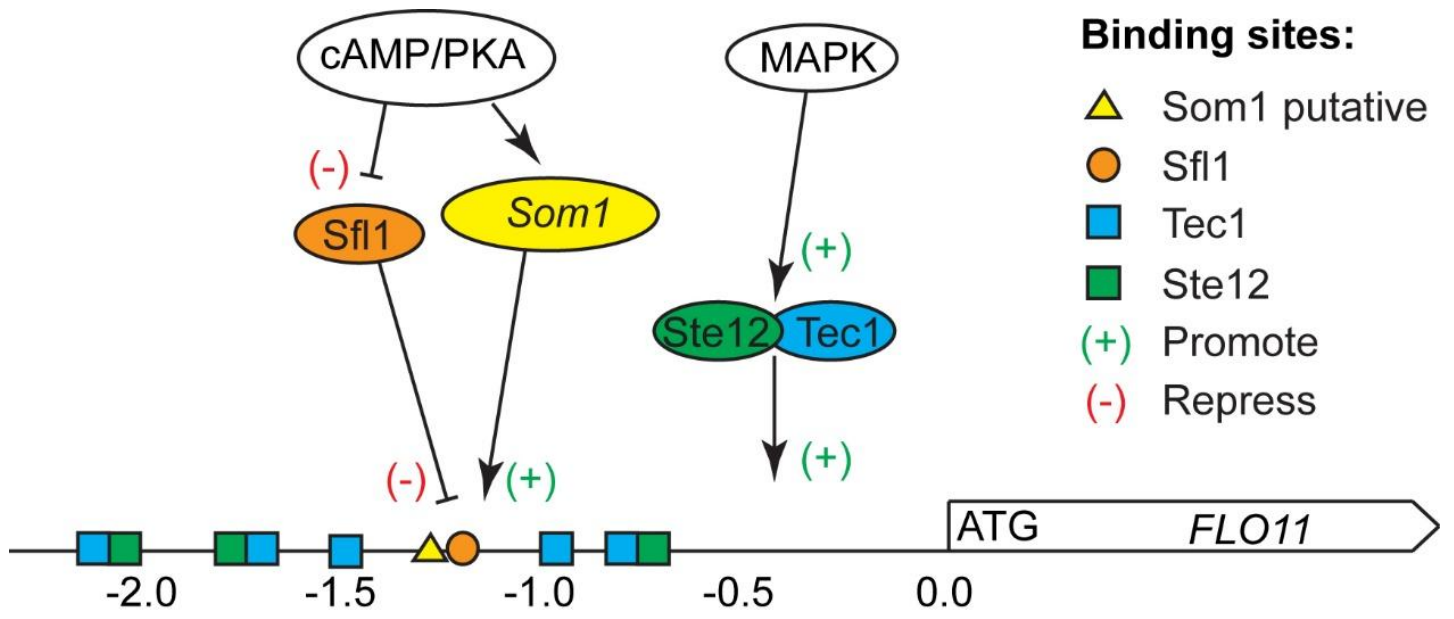

Figure 47. Som1 might directly bind to the promoter of FLO11 in S. cerevisiae. Adhesion of $S$. cerevisiae requires the expression of FLO11. The expression of FLO11 is controlled by the cAMP/PKA and MAPK pathways. The complex of Ste12 and Tec1 promotes the expression of FLO11, whereas Sfl1 represses the expression of it. In yeast, Som1 might directly bind to the promoter of FLO11 for activation. This figure is modified from (Octavio et al., 2009). 


\subsubsection{Vta3 activate adhesion through repressing the negatively acting SFL1 in S. cerevisiae}

The transcription factor Vta3 harbours the winged helix-turn-helix domain which is highly conserved between filamentous fungi. Vta3 together with Ssn6 and Tup1 might indirectly activate FLO1 and FLO11 genes via repressing the expression of SFL1. Therefore, the expression of FLO1 and FLO11 is rescued in the yeast strain expressing VTA3. In S. cerevisiae, FLO11 is directly controlled by Flo8, Ste12, Tec1, and Sfl1. Flo8, Ste12, and Tec1 play a role in activation, whereas Sfl1 is involved in inhibiting the expression of FLO11. Ste12 together with Tec1 regulated by the MAPK pathway cannot activate FLO11 when lacking FLO8. However, this complex could stimulate the expression of FLO11 when both, FLO8 and SFL1, are absent. Sfl1 is involved in the repression of related flocculation genes and binds to the promoter region of FLO11 (Conlan \& Tzamarias, 2001; Ansanay et al., 2007). Rfx1 physically interacts with the composite of Ssn6 and Tup1. This complex inhibits the expression of SFL1. (Huang et al., 1998; Conlan \& Tzamarias, 2001). Additionally, Flo8 and the complex of Ste12 and Tec1 bind to the promoter of FLO11 for activating, whereas Sfl1 binds to that promoter for suppressing the expression (Rupp et al., 1999; Conlan \& Tzamarias, 2001; Octavio et al., 2009). Defects in either Flo8 or Ste12/Tec1 prevent the expression of FLO11 because of the function of Sfl1 (Rupp et al., 1999). Lacking Sfl1 in the deletion of FLO8 might explain why the yeast strain expressing VTA3 can reprogram adhesion (Figure 48). 


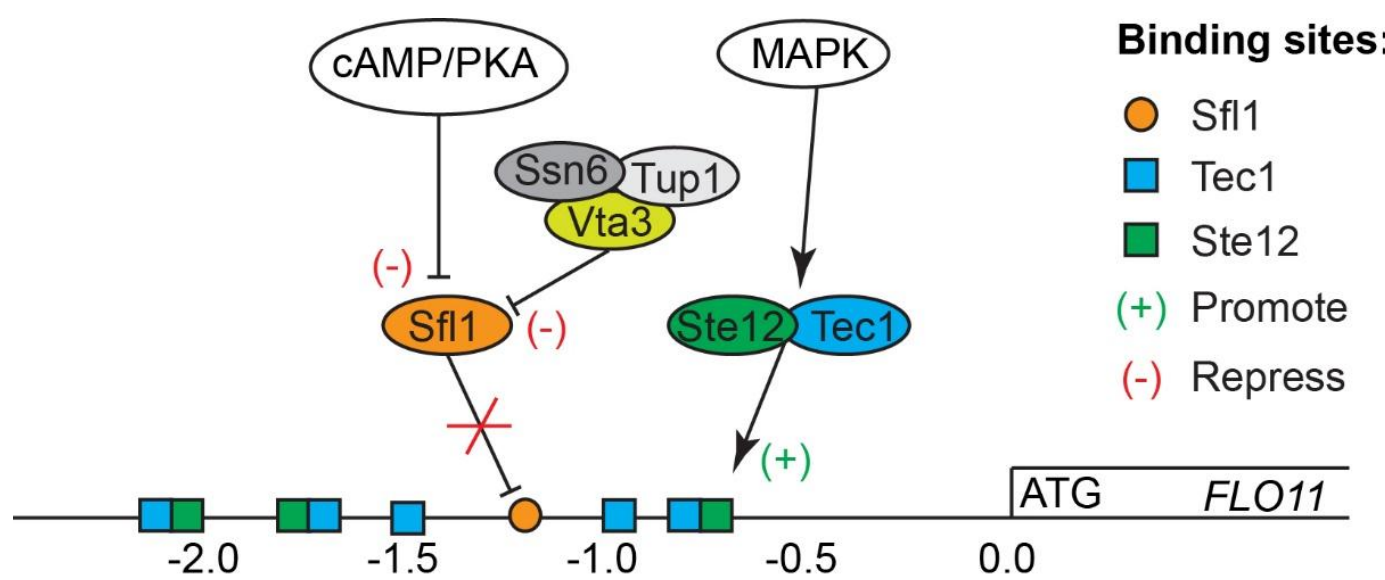

Figure 48. Vta3 activates FLO11 via repressing the expression of SFL1. Adhesion of $S$. cerevisiae requires the expression of FLO11. The expression of FLO11 is controlled by cAMP/PKA and MAPK pathways. Flo8, Sfl1, and Ste12 directly bind to the promoter of FLO11. The complex of Vta3, Ssn6, and Tup1 might repress the expression of SFL1. The expression of FLO1 can be activated by the complex of Ste12 and Tec1. This figure is modified from (Octavio et al., 2009).

\subsection{The Transcription factors Som1 and Vta3 promote fungal development and virulence}

Plant infection requires adhesive proteins in several stages of the host-fungus interaction (Braun \& Howard, 1994; Hostetter, 2000). In this study, we show that the transcription factors Som1 and Vta3 are important for morphologies and plant infection at different stages and control the expression of putative transcription factors for adhesion.

\subsubsection{Som1 and Vta3 control transcription factors for adhesion}

Som1 controls adhesion and root penetration. Both, Som1 and Vta3, promote conidiation, microsclerotia formation, and virulence. They regulate transcription factors which are involved in fungal development, virulence and adhesion in $V$. dahliae. These factors were also shown to be able to restore adhesion in the FLO8-deficient S. cerevisiae (Tran et al., 2014). Phenotypes of Som 1 and Vta3 could be explained by the functions of VTA gene. Som 1 and Vta3 might control microsclerotia and pigmentation via the regulation of VTA1. The transcription factor Vta1 encodes a putative Zn(II)2Cys6 domain. Its homolog in Fusarium fujikoroi, Fsr6, is essential for pigmentation of perithecia (Studt et al., 2012). In Verticillium, Vta1 is located in melanin biosynthetic cluster of genes. It is upregulated in microsclerotia activation medium (Duressa et al., 2013). Som1 and 
Vta3 might control conidiation, virulence via promoting VTA2. Vta2 is highly conserved in filamentous fungi (Tran et al., 2014). Its homolog in M. grisea plays important roles in conidiation, appressorium formation, and pathogenicity (Odenbach et al., 2007). In Verticillium, the transcription factor for adherence Vta2 is involved in conidia formation, stress response, virulence. It also controls the expression of 270 genes including putative target genes for adhesion, conidiation, oxidative stress response, and virulence. Especially, Vta2 controls the expression of $A B A 1$ and CON8 which are essential for conidia formation (Tran et al., 2014). It also promotes the expression of virulence factor NLP2 which is reported to play a role in growth, sporulation, and virulence (Santhanam et al., 2013; Tran et al., 2014). These conidial and virulence factors are down-regulated in deletions of VTA3 and SOM1. Som1 might regulate oxidative stress response via controlling Vta2. Moreover, Vta2 is needed for oxidative stress response and regulate the expression of genes which are related to oxidative stress response such as CPX2 and SOD3 (Tran et al., 2014), which are also regulated by Som1. Som1 perhaps promotes growth in medium with galactose as carbon-source though controlling the expression of VTA6, a putative CreA regulator, which is the negative regulator mediating carbon catabolism repression in A. nidulans (Strauss et al., 1999). CreA is supposed to act in a similar way to the general repressor Tup1 and Ssn6 to suppress the synthesis of enzymes which are involved in the catabolism of carbon sources (Panozzo et al., 1998). Taken together, Som1 and Vta3 might promote fungal development and virulence by regulating VTA genes (Figure 49). 


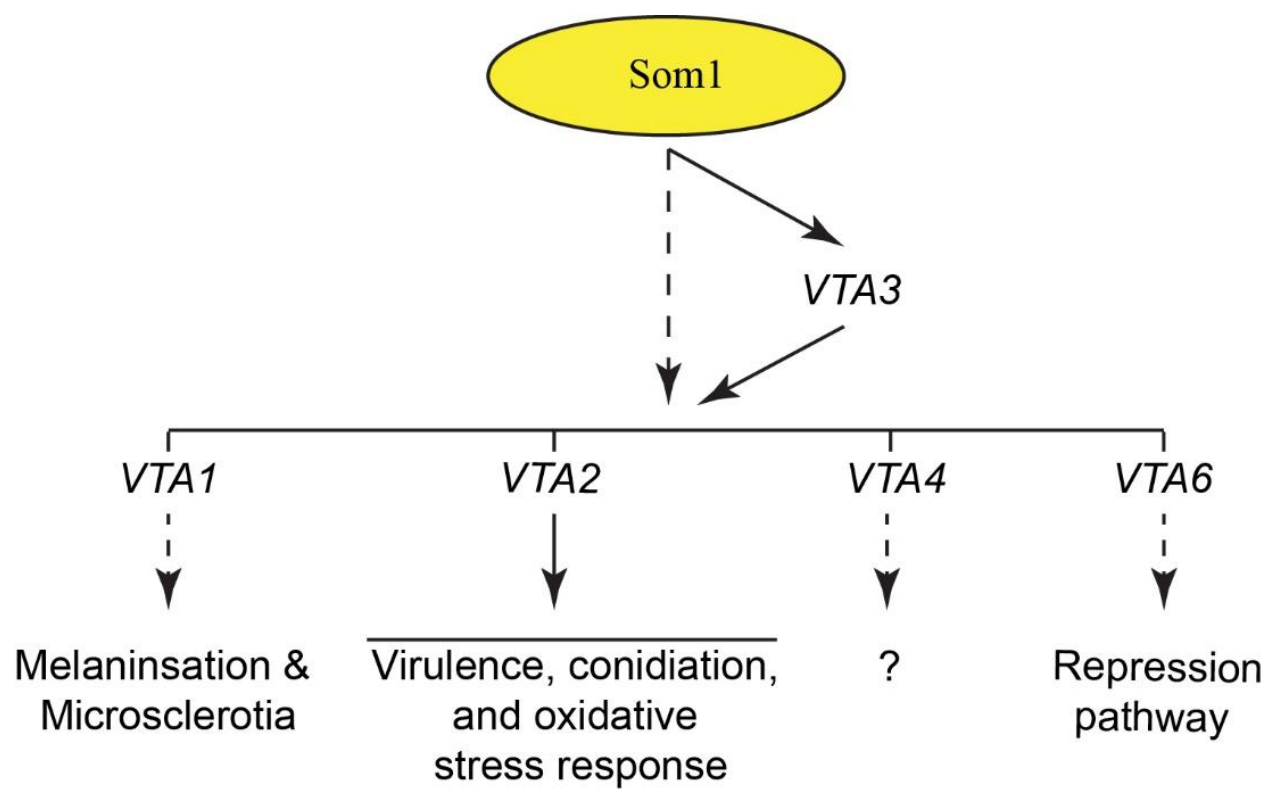

Figure 49. Som 1 and Vta3 control fungal development and virulence by regulation of VTA genes. Som1 regulates the expression of VTA3. Both, Som1 and Vta3, control the expression of VTA1, VTA2, VTA4, and VTA6 genes. Som1 might regulate the VTA genes directly or indirectly via VTA3. Vta1 might be involved in microsclerotia formation and melanisation. Vta2 is essential for conidiation and virulence. Vta6 might join the repression pathway to control genes which are involved in the catabolism of carbon sources. The solid arrows show the presented data and the result of previous studies. The dashed lines indicate putative function.

\subsubsection{Som 1 controls adhesion and penetration in V. dahliae}

In $V$. dahliae, Som1 is essential for adhesion and penetration on plant roots and regulates the expression of adhesion factors such as FAS1, MAD1, MAD2, $S N O D 1$, and PLS1. A lack of these proteins might explain why the deletion of SOM1 is defective in adhesion and penetration on plant roots. Homologs of FAS1 in Drosophila, M. oryzae, and Rhodobacter sphaeroides encode fasciclin domain proteins containing a GPI signal. They are required for conidial adhesion and appressorium turgor formation (Clout et al., 2003; Liu et al., 2009; Moody \& Williamson, 2013). Mad1 and Mad2 in Metarhizium anisopliae are involved in adhesion. The disruption of MAD1 blocks adhesion of $M$. anisopliae to insects, whereas MAD2 mutant impedes the adherence to plant surfaces (Wang \& St Leger, 2007). The counterpart of Snod1 in M. grisea is essential for the formation of adhesion structures such as appressoria. The SNODPROT1 deletion strain is impaired in the formation of appressoria which are necessary for adhesion in Magnaporthe (Jeong et al., 2007). In V. dahliae, Pls1 was reported to play a role in formation of penetration pegs. It is required for the initial colonisation of cotton roots. The deletion 
strain of PLS1 is unable to produce hyphopodia and penetration pegs and loses virulence (Zhao et al., 2016). Studies of Pls1 homologs in other fungi also show that homologs of Pls1 are required for appressoria formation and penetration of plant surfaces (Clergeot et al., 2001; Gourgues et al., 2004). It is not clear if the inability of the Som1 deletion strain to adhere to plants is caused by the down-regulation of one of these factors or if it is a combination of different factors involved in fungal adhesion. To answer this question, further studies on the different proteins and their function in Verticillium would be required.

\subsubsection{Som1 and Vta3 promote pathogenicity}

Som1 and Vta3 are required for virulence and sequential steps of root penetration and colonisation. Som1 promotes the expression of adhesion factors which might be involved in the penetration of plant roots. Additionally, Som1 controls the expression of VTA3 and together with Vta3 regulates the expression of VTA2 and several virulence factors as $N L P 2, C A P 20$, and $P R Y 1$. Therefore, the lack of Vta2 and these virulence factors might explain why the deletion strains of SOM1 and VTA3 are defective in plant infection. Vta2 is needed for root colonisation and tomato infection. The absence of VTA2 causes a block in root colonisation and pathogenicity in V. dahliae (Tran et al., 2014). Even though Vta2 and Vta3 have a different function in microsclerotia formation, they play a similar function in root colonisation. Both deletion strains of SOM1 and VTA3 show defects in tomato root colonisation as observed in the deletion of Vta2.

NLP2 encodes a cytotoxic necrosis and ethylene-like protein which is expressed during the colonisation of plant roots by the fungus. It was reported to contribute to the pathogenicity and disease symptoms of $V$. dahliae on tomatoes (Santhanam et al., 2013). Plants infected by deletion strains of SOM1 and VTA3 have no disease symptom. The homolog of Cap20 in Colletotrichum gloeosporioides is essential for appressoria formation and virulence. CAP2O is expressed during appressoria formation. Deletion of CAP20 causes defects in the colonisation of tomato fruits (Hwang et al., 1995). Both Som1 and Vta3 are required for root infection. Deletion strains of SOM1 and VTA3 were not isolated from plant hypocotyl. The deletion strains might not be able to enter plant root or spread in the plant. 
The expression of $P R Y 1$ is significantly down-regulated in the deletions of SOM1, VTA3, and VTA2. The Pry1 counterpart in F. oxysporum is required for virulence in mammalian hosts (Prados-Rosales et al., 2012). Som1 and Vta3 may possibly regulate pathogenicity via controlling genes which are related to virulence.

Moreover, our study show that Cyc8 was identified by LC-MS/MS analyses after enrichment of VTA3:GFP by GFP-trap. Vta3 might physically interact with Cyc8 to control virulence in $V$. dahliae. The homolog of Vta3 in S. cerevisiae physically interacts with Ssn6 which is a counterpart of Cyc8 (Huang et al., 1998). SsnF, a homolog of Cyc8 in A. fumigatus, was reported to interact with Fbx15 which is essential for virulence (Johnk et al., 2016). In V. dahliae, Cyc8 is reported to play a major role in virulence. The deletion strain of $\mathrm{CYC8}$ is impaired in virulence in cotton (Li et al., 2015b). It is not clear whether the complex of Vta3 and Cyc8 controls directly or indirectly the virulence by regulating growth and microsclerotia formation. The function of virulence factors which were found in protein assays has not been studied yet. To understand more about the virulence mechanism of $V$. dahliae, the roles of putative virulence factors should be investigated.

\subsubsection{Som1 and Vta3 are essential for conidia and microsclerotia formation}

After plant root infection, pathogenic fungi can distribute within the plant by the formation of conidia which are also required to spread the pathogen on the field (Pegg \& Brady, 2002; Berlanger \& Powelson, 2005). Conidia are formed in a specialised structure, called the conidiophore. Each conidiophore produces several phialides on which a number of conidia develop. This unit of conidia on a phialide, we called conidiospore clusters. We found that Som1 and Vta3 are necessary for conidiation in general and also for conidiospore cluster formation. Conidia formation is reduced in both deletions, whereas the number of conidiospore clusters is only decreased in the deletion strain of SOM1.

The counterpart of Vta3 in F. graminearum is essential for spore shape (Min et al., 2014). However, in this study, the shape of conidia of the VTA3 deletion strain was not changed, whereas conidiospore clusters had an abnormal shape. Both, Som1 and Vta3, regulate the expression of conidiation factors such as VTA2, CON8, and $A B A 1$. Vta2, a homolog of Con7, is essential for conidia formation and promotes the expression of CON8 and ABA1 (Tran et al., 2014). The homolog of Con8 
in Neurospora crassa was shown to be involved in sporulation and is expressed early during this process (Roberts \& Yanofsky, 1989). The counterparts of Aba1 in A. nidulans and $A$. fumigatus are needed for differentiation of conidia from vegetative hyphae (Tao \& Yu, 2011; Son et al., 2013). The absence of Con8 and Aba1 might be the cause of reduction of spore amount in deletion strains of SOM1 and VTA3. Additionally, the expression of CON6 and CON10 is significantly declined in the deletion of SOM1, whereas it does not change if VTA3 is deleted. The homolog of Con6 in $N$. crassa is required for early conidia differentiation, whereas the counterpart of Con10 is essential for later stages (Roberts \& Yanofsky, 1989). Lacking Con6 and Con10 might explain why the deletion strain of SOM1 have fewer conidiospore clusters than the wild-type.

Som1 and Vta3 do not regulate the same genes. So there seem to be overlapping regulatory functions but also divergent. Som1 controls VTA3 and regulated genes in the VTA3 deletion strain. In contrast, Som1 might have additional functions which are independent of Vta3 as the function encoded by other genes only regulated by Som1 (Figure 50).

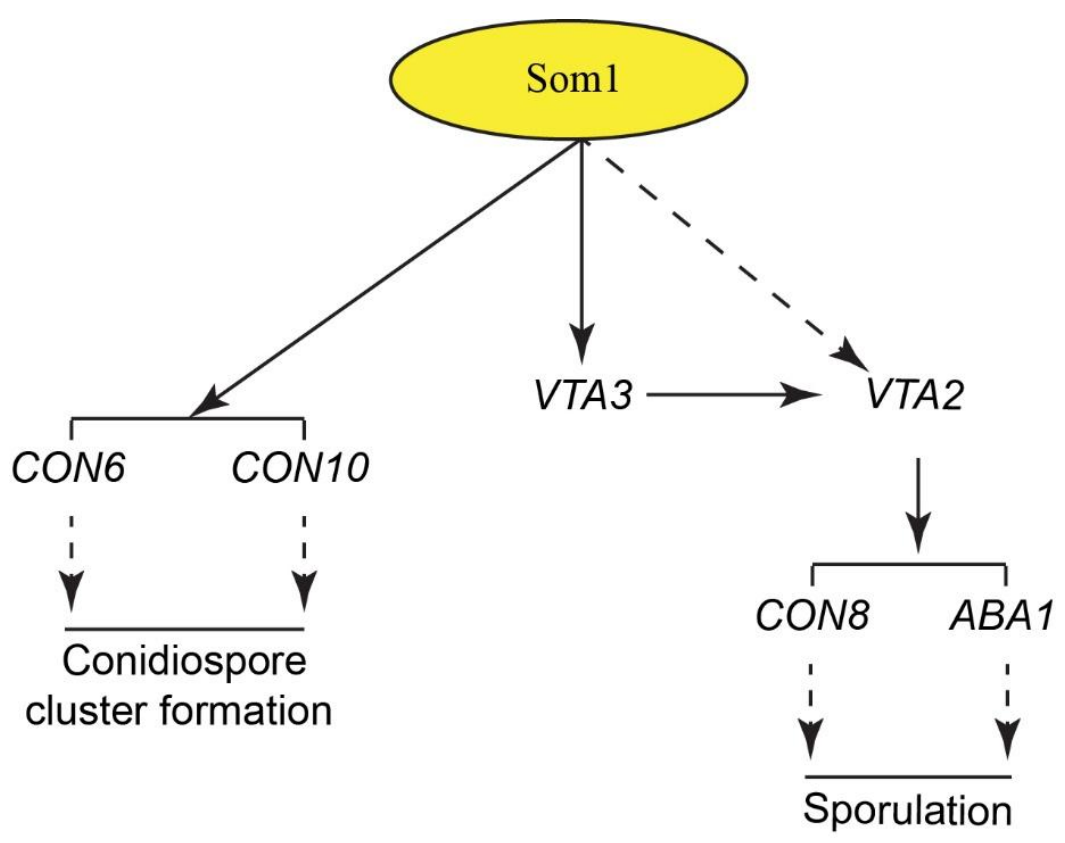

Figure 50. Model illustrating how Som1 and Vta3 can control conidia formation. Som1 regulates the expression of VTA3. Both, Som1 and Vta3, control the expression of VTA2, CON8, and $A B A 1$ genes which are essential for conidia formation. Som1 might regulate the conidia formation directly or indirectly via VTA3. CON8 and $A B A 1$ are regulated by transcription factor Vta2. Additionally, Som1 might regulate conidiospore clusters formation via controlling the expression of CON6 and CON10. 
Microsclerotia are resting structures to overwinter and also to survive several years without appropriate hosts, which are usually formed in dead plant materials and necessary for the reinfection after the winter (Pegg \& Brady, 2002). In this study, we show that both, Som1 and Vta3, support melanised cell formation. Som1 and Vta3 might be a dual control system of microsclerotia development. The deletion strain of SOM1 was not able to form resting structures, whereas the number of microsclerotia was reduced in the VTA3 deletion strain. The lacking Vdh1 might explain why no or fewer microsclerotia were developed in the deletion strains of SOM1 or VTA3 compared to the wild-type. Vdh1 was described as a key factor which is involved in the formation of melanised cells, whereas it is not required for hyphal growth. Resting structures were not detected in the deletion strain of VDH1 (Klimes \& Dobinson, 2006). Additionally, Vta3 might function together with Cyc8 in microsclerotia formation. They might control the expression of genes which are required for microsclerotia formation and pigmentation. Cyc8, an interaction partner of Vta3, was shown to play a role in the formation of microsclerotia. The deletion strain of CYC8 shows lack of melanin and fewer microsclerotia (Li et al., 2015b). Cyc8 controls the expression of genes which are involved in melanin synthesis or microsclerotia formation such as encoding laccase (VDAG_00189), conidial yellow pigment biosynthesis polyketide synthase (VDAG_00190), tetrahydroxynaphthalene reductase (VDAG_03665), and scytalone dehydratase (VDAG_03393) (Li et al., 2015b). Lack of these proteins might explain why the deletion strains of VTA3 and CYC8 are defective in microsclerotia formation. Functions of these genes have not been studied yet. To know which of them are specific for microsclerotia formation, further experiments would have to be performed.

\subsubsection{Som1 and Vta3 antagonise the oxidative stress response}

When pathogens infect host plants, they are confronted with oxidative stress and a proper response to reactive oxygen species is important to successfully colonise the plant (Tanabe et al., 2011; Youseff et al., 2012). We found that Som1 and Vta3 play antagonising roles in oxidative stress response. Som1 positively regulates oxidative stress response, whereas Vta3 negatively influences it. Oxidative stress response of fungi is regulated by several factors. It requires not only the expression of encoding genes but also to stabilize of oxidative stress tolerance proteins (Nahlik et al., 2010; Tanabe et al., 2011; 
Youseff et al., 2012). Additionally, oxidative stress tolerance needs a transcription factor which is involved in secondary metabolism (Baidya et al., 2014). Som1 probably controls oxidative stress tolerance by two different mechanisms. On the one hand, Som1 might control the expression of VEL1, which encodes a key factor of secondary metabolism (Figure 51). The counterpart of Vel1 in A. flavus, a member of velvet complex, was shown to be involved in secondary metabolism and it was essential for oxidative stress response (Baidya et al., 2014). On the other hand, Som 1 could promote the expression of genes encoding enzymes which are required for stress response (Figure 51). For example, the homolog of CPX2 in M. oryzae encodes a secreted catalase-peroxidase, whereas the counterpart of SOD3 in Histoplasma capsulatum encrypts a superoxide dismutase. Both, Cpx2 and Sod3, are controlled by Vta2 (Tran et al., 2014) and have a function in fungal defense again the oxidative stress (Tanabe et al., 2011; Youseff et al., 2012). Furthermore, the stability of transcription factors is often controlled by the COP9 signalosome (CSN) including the Ubiquitin-Proteasome system (Chamovitz, 2009; Braus et al., 2010; Nahlik et al., 2010). COP9, a multi-subunit complex, can regulate the stability of $\mathrm{VeA}$ protein and other proteins including transcription factors for oxidative stress response (Chamovitz, 2009; Braus et al., 2010; Nahlik et al., 2010). Fbx15 is induced by oxidative stress. In stress condition, Fbx15 protein is elevated 3 times when compared to normal condition (Johnk et al., 2016). It is reported to interact with three subunits of the CSN deneddylase (Nahlik et al., 2010; Johnk et al., 2016). The effect of Som 1 on the stability of proteins which are essential for oxidative stress response have not been studied yet. To understand better the oxidative stress response processes, the effect of Som1 on the stability of transcription factor and other functions of candidates for stress response have to be done. 


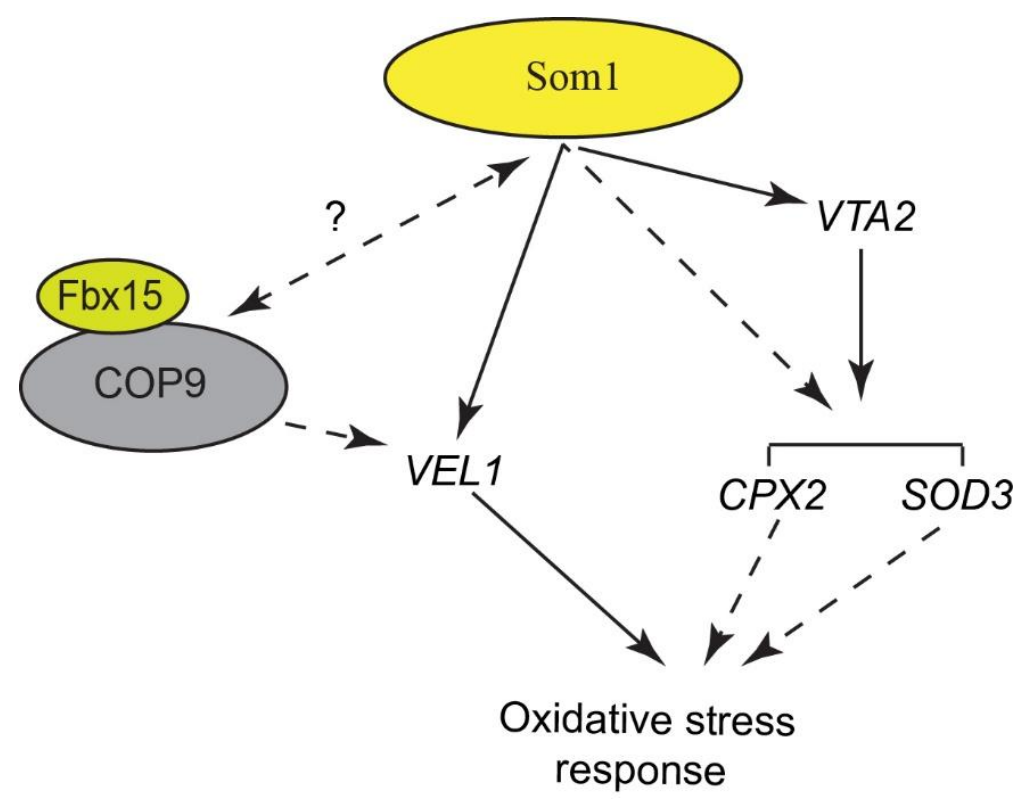

Figure 51. Model of Som1 reregulates the oxidative stress response. Som1 regulates directly or indirectly through Vta2 the expression of $C P X 2$ and $S O D 3$, required for oxidative stress response. On the other hand, Som1 might also regulate stress response and secondary metabolism pathway via synchronising VEL1.

Vta3 does not promote the expression of enzymes which are involved in oxidative stress response, however, it represses the expression of INO1. The homolog of Vta3 in S. cerevisiae, Rfx1, was reported to interact with Itc1 and Isw2 which form a multiplex to repress INO1 expression (Sugiyama \& Nikawa, 2001; Gavin et al., 2002). The disruption of either ITC1 or ISW2 results in de-repression of the INO1 expression (Sugiyama \& Nikawa, 2001). INO1 encodes an inositol3-phosphate synthase which catalyses inositol synthase (Wang et al., 2015). The overexpression of INO1 enhances the tolerance to inhibitors (Wang et al., 2015). Vta3 might interact with Itc1 and Isw2. This compound might repress the expression of INO1 to antagonise oxidative stress response (Figure 52). Lacking of Vta3 and the function of the repressor complex of INO1 might explain why the expression of INO1 and oxidative stress response are significantly increase in the deletion of VTA3. Homologs of Itc1 and Isw2 were found in V. dahliae. However, Itc1 and Isw2 were not detected in our pull-down assays neither in the list of proteins interacted with free GFP nor with Vta3:GFP. Probably the interaction of the proteins with Vta3 is week and transient to get them pulled out. It is also possible that the expression of ITC1 and ISW2 requires certain conditions such as stress inducing media to be identified in the pull-downs of Vta3:GFP. Therefore, condition in which ITC1 and ISW2 are expressed need to be examined to check the possible interaction of Vta3 
with both, Itc1 and Isw2. When these conditions are determined chromatin immunoprecipitation can be also used to possibly identify interactions between Vta3, Itc1, and Isw2.

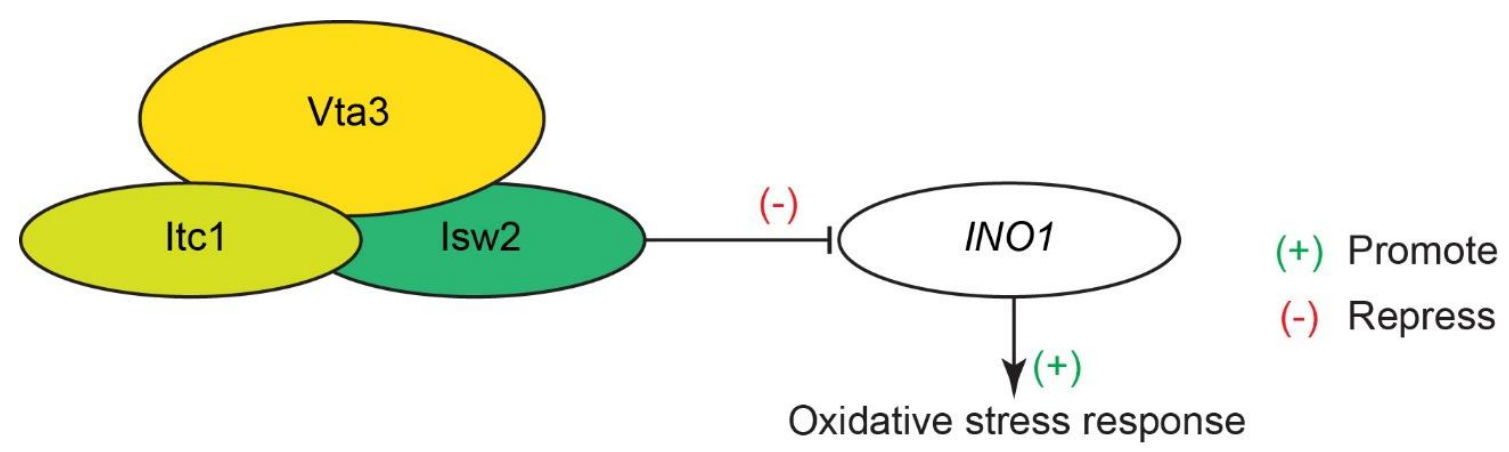

Figure 52. Vta3 can repress oxidative stress response via suppressing INO1. Vta3 might interact with Itc1 and Isw2. This complex usually suppresses the expression of INO1 which is required for oxidative stress response.

\subsubsection{Som1 and Vta3 are required for hyphal development}

Alterations in the colony phenotype might be directly connected to hyphal development and intracellular processes. We found that Som1 also functions in these processes. Som1 is required for normal hyphal branching, septum positioning, and vacuole size. The genetic mechanisms underlying hyphal branching are not well-studied in filamentous fungi. Septum formation is well studied in $A$. nidulans. Septa are produced from three major rings including actins, septin and formin (Harris, 2001). Formation of septa is a key step in hyphal differentiation (Harris, 1997; Harris, 2001). The formation of septa requires several factors such as SepH, actin, the septin AspB, and the formin SepA (Harris, 2001). Recently, DipA of $A$. nidulans was reported to be required for septa positioning as well (Schinke et al., 2016). The deletion strain of DIPA showed an increased number of septa compared to the wild-type (Schinke et al., 2016). This phenotype is similar to what was observed in the SOM1 deletion strain. The deletion strain had more septa than the wild-type strain. A homolog of DipA is present in $V$. dahliae but its functions have not been studied so far. It is possible that Som1 and Dip1 directly or indirectly interact and/or act as a dual control system for septum positioning.

Vacuoles are essential for cellular differentiation. They were reported to play a major role in cell differentiation during the formation of appressoria which are specialised penetration structures in C. albicans and U. maydis (Weber, 2002). 
Vacuoles help to expand the surface of fungal tips in order to support appressorium formation via degrading lipid stores which generate osmotically active metabolites (Veses et al., 2008). This process supports the forced entry of the fungal penetration peg into the plant epidermis (Weber et al., 2001). Additionally, vacuoles are essential for stress response (Weber, 2002; Palmer et al., 2003; Veses et al., 2008). In this study, we found that Som1 is required for microsclerotia formation, hyphopodia formation, root penetration, and virulence in $V$. dahliae. These phenotypes might be explained by the defect of vacuole size. The small vacuole size might be related to the inability of the SOM1 deletion strain to form hyphopodia and microsclerotia and to respond to oxidative stress and virulence. Fatty acids are major components of phospholipid and glycolipid compounds which play a key role in the structure and functioning of membranes such as vacuoles and plasma membranes (Michaillat \& Mayer, 2013; van Zutphen et al., 2014). Lack of fatty acid might cause the defect in vacuole size. The expression of genes encoding fatty acid synthase S-acetyltransferase which catalyses fatty acid synthesis is significantly down-regulated in the deletion of SOM1 (Table 4). The hyphal development in Verticillium has not yet been studied in detail. To understand more about that process further studies on hyphal development and protein regulated hyphal development would have to be performed.

\subsection{AfSom1 and VdSom1 fulfil similar functions in plant and human pathogens}

Plant and human pathogenic fungi have massively attracted attention because they have some common pathogenicity factors. Studies of the function of these factors in both, plant and human, pathogens help to understand aspects of infection and virulence ( $\mathrm{Yu}$ et al., 2017). There are several factors which are reported to play a major role in virulence in plant pathogens and counterparts are involved in pathogenicity in human pathogenic fungi (Yu et al., 2017). They are useful for antifungal strategies and development of new fungicides to control pathogens (Fones et al., 2017; Yu et al., 2017). We recently described that the transcription factor AfSomA in A. fumigatus plays important roles in adhesion, conidia formation and virulence in the human pathogenic fungus (Lin et al., 2015). 
In this study, we show that AfSomA and VdSom1 fulfil similar functions in the human pathogenic $A$. fumigatus as in the plant pathogen $V$. dahliae. AfSom 1 does not only rescues growth, adhesion, conidia formation, and virulence but also reprograms microsclerotia formation in $V$. dahliae, a process which does not exist in A. fumigatus. This pathogenicity factor of a plant invader and its counterpart of human pathogenic fungi is highly conserved. The result of this study could help to understand penetration and virulence pathways not only in plant pathogenic fungi but also in human pathogens. Nowadays, antifungal azole is widely used in agriculture and also the clinic to control pathogenic fungi (Fones et al., 2017). However, some new pathogens became resistant against this fungicide. A. fumigatus, which causes lung disease, does not respond to azole treatment (Fones et al., 2017). Furthermore, infection of this fungus is often underdiagnosed and treated rather late. Therefore, the effect of fungicides is often limited, and up to $80 \%$ of patients infected by $A$. fumigatus die (Fones et al., 2017). It is necessary to develop new fungicides to control this pathogenic fungus. In $V$. dahliae, controlling the expression of SOM1 might stop the fungus from penetrating plant roots and block virulence, whereas manipulation of the expression of VTA3 could prevent the development of the pathogen in planta to reduce disease. Som1 and Vta3 might be putative targets for antifungal intervention. A new effective fungicide which can treat new pathogenic fungi and $A$. fumigatus can help us save million euro yearly.

In summary, Som1 is required for adhesion on abiotic surfaces, penetration, and colonisation of the root surface, whereas Vta3 is needed for colonisation only. Both, Som1 and Vta3, are essential for conidia and microsclerotia formation in $V$. dahliae. Especially, the SOM1 deletion strain was unable to produce microsclerotia. Som1 promotes oxidative stress response, whereas Vta3 inhibits this reaction. Additionally, Som1 is required for hyphal development including aerial hyphae, hyphal growth on agar plates, hyphal branching, septum position, and normal vacuole size where Vta3 is essential for hyphal growth on agar plates. (Figure 53). 


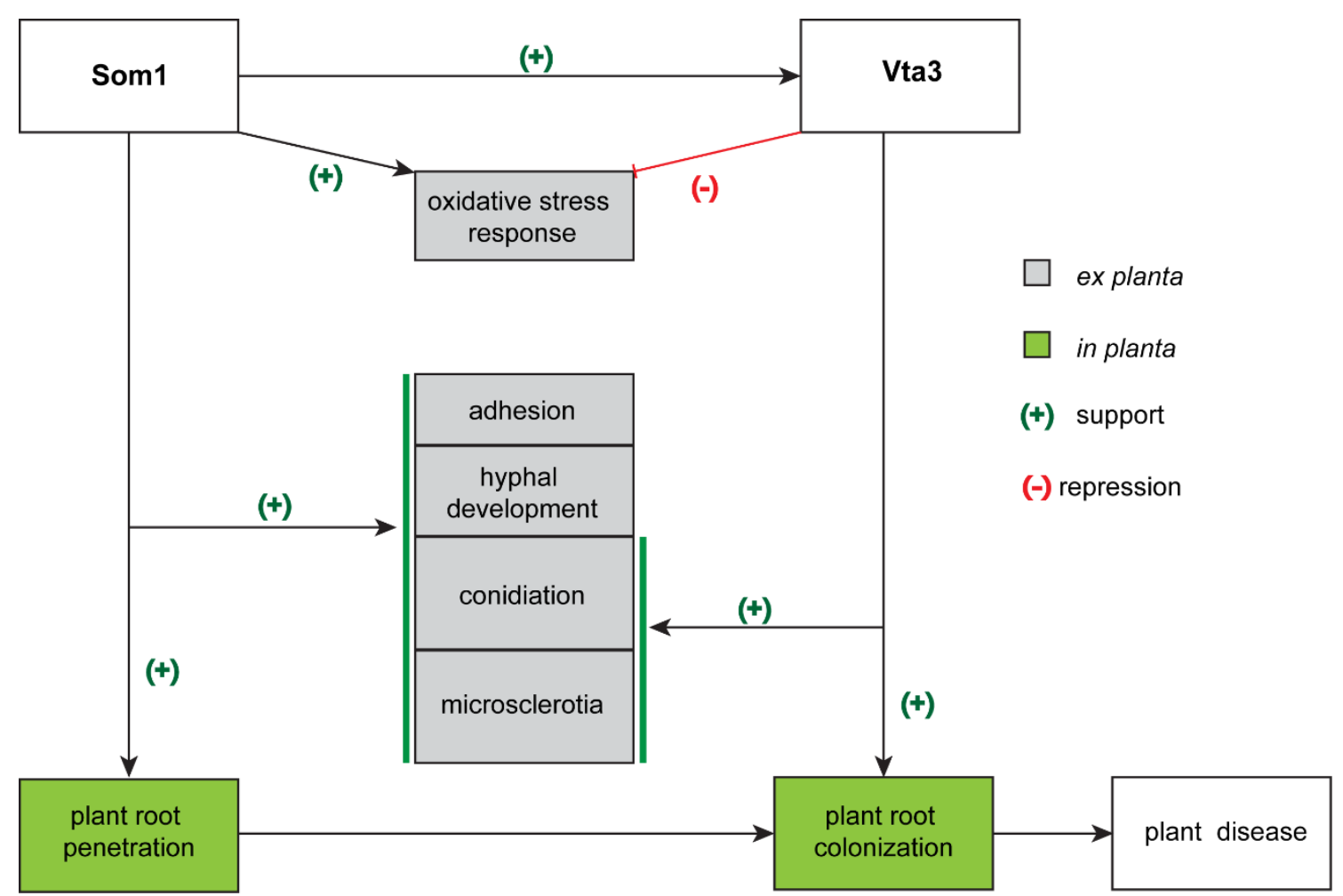

Figure 53. The functions of Som 1 and Vta3 in V. dahliae. Som 1 is a transcription factor which supports adhesion, conidiation, microsclerotia formation, oxidative stress response, and plant root penetration. It controls the expression of VTA3 which encodes a protein that promotes conidiation, microsclerotia formation, and plant root colonization and represses oxidative stress response.

\subsection{Outlook}

In this study, we found that the nuclear transcription factors of Verticillium dahliae Som1 and Vta3, which can rescue adhesion in a flo8-deficient Saccharomyces cerevisiae strain, are controlling sequential steps in the infection of a plant host. Som1 might directly bind to the promoter of adhesive genes for activation, whereas Vta3 indirectly induces FLO1 and FLO11 genes by repressing the expression of SFL1. However, the mechanisms of other adhesion candidates of the yeast screen which can reprogram adherence in non-adhesion yeast and their functions in V. dahliae are unclear (Tran et al., 2014). Especially, the transcription factors of adhesion such as VTA1, VTA4, and VTA6 which were down-regulated in deletion strains of SOM1 and VTA3 have not been studied yet.

Both, Som1 and Vta3, control several similar phenotypes and virulence. Som1 regulates the expression of VTA3. Therefore, Som1 might indirectly promote cellular development and virulence via Vta3. To better understand the relation of 
Vta3 to Som1, the expression of VTA3 under the control of a constitutive promoter in the deletion strain of SOM1 could be examined. In such an experiment Vta3independent functions of Som1 could be discriminated from effects resulting from a reduced expression of VTA3 in the SOM1 deletion strain.

Our proteomic data indicate that twenty abundant proteins were significantly reduced in the deletion of SOM1 including Cap20 and Snodprot1. They have not been studied yet and might play important roles in $V$. dahliae. Additionally, three putative uncharacterized proteins were also abundant in the wild-type, but they are not found in deletion of SOM1. They might fulfil critical functions in $V$. dahliae. The function of Cap20, Snod1, and unknown proteins which were significantly reduced in the SOM1 deletion strain need to be investigated further.

Therefore, future work will focus on adhesion factors, cellular connections and target genes which are controlled by Som 1 and Vta3 and are needed to better understand and control plant and human pathogens more efficiently. 


\section{References}

Alberti-Segui C, Morales AJ, Xing H, Kessler MM, Willins DA, Weinstock KG, Cottarel G, Fechtel K, Rogers B (2004) Identification of potential cell-surface proteins in Candida albicans and investigation of the role of a putative cellsurface glycosidase in adhesion and virulence. Yeast 21: 285-302.

Amberg DC, Burke D, Strathern JN, Burke D, Cold Spring Harbor Laboratory (2005) Methods in yeast genetics : a Cold Spring Harbor Laboratory course manual. Cold Spring Harbor, N.Y.: Cold Spring Harbor Laboratory Press.

Ansanay Galeote V, Alexandre H, Bach B, Delobel P, Dequin S, Blondin B (2007) Sfl1p acts as an activator of the HSP30 gene in Saccharomyces cerevisiae. Current Genetics 52: 55-63.

Baidya S, Duran RM, Lohmar JM, Harris-Coward PY, Cary JW, Hong SY, Roze LV, Linz JE, Calvo AM (2014) VeA Is Associated with the Response to Oxidative Stress in the Aflatoxin Producer Aspergillus flavus. Eukaryotic Cell 13: 1095-1103.

Beckerman JL, Ebbole DJ (1996) MPG1, a gene encoding a fungal hydrophobin of Magnaporthe grisea, is involved in surface recognition. Mol Plant Microbe Interact 9: 450-456.

Bennett JW, Lasure LL (1991) Growth media. San Diego: Academic.

Berlanger I and Powelson ML (2005) Verticillium wilt. The Plant Health Instructor. doi: 10.1094/PHI-I-2000-0801-01.

Bonfim-Mendonca PS, Tobaldini FK, Capoci IRG, Negri M, Kioshima ES, Svidzinski TIE (2015) Quantitative expression of ALS family genes of Candida albicans in the experimental cervical epithelial infections. Mycoses 58: 213-213.

Brandt WH (1967) Influence of near-ultraviolet light on hyphal elongation in Verticillium. Mycologia 59: 736-739.

Braun EJ, Howard RJ (1994) Adhesion of Fungal Spores and Germlings to HostPlant Surfaces. Protoplasma 181: 202-212.

Braus GH, Grundmann O, Bruckner S, Mosch HU (2003) Amino acid starvation and Gcn4p regulate adhesive growth and FLO11 gene expression in Saccharomyces cerevisiae. Mol Biol Cell 14: 4272-4284. 
Braus GH, Irniger S, Bayram Ö (2010) Fungal development and the COP9 signalosome. Curr Opin Microbiol 13: 672-676.

Bugeja HE, Hynes MJ, Andrianopoulos A (2010) The RFX protein RfXA is an essential regulator of growth and morphogenesis in Penicillium marneffei. Eukaryot Cell 9: 578-591.

Calderone R (1998) The INT1 of Candida albicans. Trends in Microbiology 6(8): 300-301; discussion 302-303.

Chamovitz DA (2009) Revisiting the COP9 signalosome as a transcriptional regulator. Embo Reports 10: 352-358.

Clergeot PH, Gourgues M, Cots J, Laurans F, Latorse MP, Pepin R, Tharreau D, Notteghem JL, Lebrun MH (2001) PLS1, a gene encoding a tetraspanin-like protein, is required for penetration of rice leaf by the fungal pathogen Magnaporthe grisea. Proc Natl Acad Sci U S A 98: 6963-6968.

Clout NJ, Tisi D, Hohenester E (2003) Novel Fold Revealed by the Structure of a FAS1 Domain Pair from the Insect Cell Adhesion Molecule Fasciclin I. Structure 11: 197-203.

Conlan RS, Tzamarias D (2001) Sfl1 functions via the co-repressor Ssn6-Tup1 and the cAMP-dependent protein kinase Tpk21. Journal of Molecular Biology 309: 1007-1015.

Cotter G, Kavanagh K (2000) Adherence mechanisms of Candida albicans. Br J Biomed Sci 57: 241-249.

Covert SF, Kapoor P, Lee M-h, Briley A, Nairn CJ (2001) Agrobacterium tumefaciens-mediated transformation of Fusarium circinatum. Mycological Research 105: 259-264.

Dranginis AM, Rauceo JM, Coronado JE, Lipke PN (2007) A biochemical guide to yeast adhesins: Glycoproteins for social and antisocial occasions. Microbiology and Molecular Biology Reviews 71: 282-294.

Duressa D, Anchieta A, Chen D, Klimes A, Garcia-Pedrajas MD, Dobinson KF, Klosterman SJ (2013) RNA-seq analyses of gene expression in the microsclerotia of Verticillium dahliae. BMC Genomics 14: 607. 
Emery P, Durand B, Mach B, Reith W (1996) RFX proteins, a novel family of DNA binding proteins conserved in the eukaryotic kingdom. Nucleic Acids Res 24: 803-807.

Epstein L, Nicholson R (2016) Adhesion and Adhesives of Fungi and Oomycetes. In: Smith AM ed. Biological Adhesives. Cham: Springer International Publishing, 25-55.

Eynck C, Koopmann B, Grunewaldt-Stoecker G, Karlovsky P, von Tiedemann A (2007) Differential interactions of Verticillium longisporum and $V$-dahliae with Brassica napus detected with molecular and histological techniques. European Journal of Plant Pathology 118: 259-274.

Fan Y, He H, Dong Y, Pan H (2013) Hyphae-specific genes HGC1, ALS3, HWP1, and ECE1 and relevant signaling pathways in Candida albicans. Mycopathologia 176: 329-335.

Fichtner L, Schulze F, Braus GH (2007) Differential Flo8p-dependent regulation of FLO1 and FLO11 for cell-cell and cell-substrate adherence of S-cerevisiae S288c. Molecular microbiology 66: 1276-1289.

Finn RD, Coggill P, Eberhardt RY, Eddy SR, Mistry J, Mitchell AL, Potter SC, Punta M, Qureshi M, Sangrador-Vegas A (2016) The Pfam protein families database: towards a more sustainable future. Nucleic Acids Res 44: 279-285.

Fones HN, Fisher MC, Gurr SJ (2017) Emerging Fungal Threats to Plants and Animals Challenge Agriculture and Ecosystem Resilience. Microbiology Spectrum 5.

Fradin EF, Zhang Z, Juarez Ayala JC, Castroverde CD, Nazar RN, Robb J, Liu CM, Thomma BP (2009) Genetic dissection of Verticillium wilt resistance mediated by tomato Ve1. Plant Physiol 150: 320-332.

Fu Y, Ibrahim AS, Sheppard DC, Chen YC, French SW, Cutler JE, Filler SG, Edwards JE, Jr (2002) Candida albicans Als1p: an adhesin that is a downstream effector of the EFG1 filamentation pathway. Mol Microbiol 44: 61-72.

Gajiwala KS, Burley SK (2000) Winged helix proteins. Curr Opin Struct Biol 10: 110-116. 
Gale CA, Bendel CM, McClellan M, Hauser M, Becker JM, Berman J, Hostetter MK (1998) Linkage of adhesion, filamentous growth, and virulence in Candida albicans to a single gene, INT1. Science (New York, N.Y.) 279: 1355-1358.

Gavin AC, Bosche M, Krause R, Grandi P, Marzioch M, Bauer A, Schultz J, Leutwein C, Bouwmeester T, Kuster B (2002) Functional organization of the yeast proteome by systematic analysis of protein complexes. Faseb Journal 16: A523-A523.

Geoghegan IA, Gurr SJ (2016) Chitosan Mediates Germling Adhesion in Magnaporthe oryzae and Is Required for Surface Sensing and Germling Morphogenesis. PLoS Pathog 12: e1005703.

Gietz RD, Schiestl RH (2007) High-efficiency yeast transformation using the LiAc/SS carrier DNA/PEG method. Nat Protoc 2: 31-34.

Gourgues M, Brunet-Simon A, Lebrun MH, Levis C (2004) The tetraspanin BcPls1 is required for appressorium-mediated penetration of Botrytis cinerea into host plant leaves. Mol Microbiol 51: 619-629.

Guebel DV, Nudel CB (1994) Antagonism between growth and flocculation inPichia stipitis NRRL Y-7124:Influence of $\mathrm{Ca}+2$ and $\mathrm{Mg}+2$ ions. Biotechnology Letters 16: 143-148.

Haimes J, Kelley M (2014) Demonstration of $\Delta \Delta \mathrm{Cq}$ calculation method to computer relative gene expression from qPCR data. [WWW document] URL http://dharmacon.gelifesciences.com/uploadedfiles/resources/delta-cqsolaris-technote.pdf. [published August 2014].

Harris SD (1997) The duplication cycle in Aspergillus nidulans. Fungal Genet Biol 22: $1-12$.

Harris SD (2001) Septum formation in Aspergillus nidulans. Curr Opin Microbiol 4: 736-739.

Hostetter MK (2000) RGD-mediated adhesion in fungal pathogens of humans, plants and insects. Curr Opin Microbiol 3: 344-348.

Hoyer LL (2001) The ALS gene family of Candida albicans. Trends in Microbiology 9: $176-180$.

Hoyer LL, Payne TL, Bell M, Myers AM, Scherer S (1998) Candida albicans ALS3 and insights into the nature of the ALS gene family. Current Genetics 33: 451-459. 
Huang MX, Zhou Z, Elledge SJ (1998) The DNA replication and damage checkpoint pathways induce transcription by inhibition of the Crt1 repressor. Cell 94: 595-605.

Hwang CS, Flaishman MA, Kolattukudy PE (1995) Cloning of a gene expressed during appressorium formation by Colletotrichum gloeosporioides and a marked decrease in virulence by disruption of this gene. Plant Cell 7: 183-193.

Inderbitzin P, Bostock RM, Davis RM, Usami T, Platt HW, Subbarao KV (2011a) Phylogenetics and taxonomy of the fungal vascular wilt pathogen Verticillium, with the descriptions of five new species. PLoS One 6: e28341.

Inderbitzin P, Davis RM, Bostock RM, Subbarao KV (2011b) The ascomycete Verticillium longisporum is a hybrid and a plant pathogen with an expanded host range. PLoS One 6: e18260.

Inoue H, Nojima H, Okayama H (1990) High efficiency transformation of Escherichia coli with plasmids. Gene 96: 23-28.

Isaac I (1947) A comparative study of pathogenic isolates of Verticillium. . Trans. Br. Mycol. Soc 32: 137-157.

Janus D, Hoff B, Hofmann E, Kück U (2007) An efficient fungal RNA-silencing system using the DsRed reporter gene. Applied and Environmental Microbiology 73: 962-970.

Jeong JS, Mitchell TK, Dean RA (2007). The Magnaporthe grisea snodprot1 homolog, MSP1, is required for virulence. FEMS Microbiol Lett 273: 157-165.

Johnk B, Bayram Ö, Abelmann A, Heinekamp T, Mattern DJ, Brakhage AA, Jacobsen ID, Valerius O, Braus GH (2016) SCF Ubiquitin Ligase F-box Protein Fbx15 Controls Nuclear Co-repressor Localization, Stress Response and Virulence of the Human Pathogen Aspergillus fumigatus. PLoS Pathog 12: e1005899.

Jyothishwaran G, Kotresha D, Selvaraj T, Srideshikan SM, Rajvanshi PK, Jayabaskaran C (2007) A modified freeze-thaw method for efficient transformation of Agrobacterium tumefaciens. Current Science 93: 770-772.

Kim HY, Lee SB, Kang HS, Oh GT, Kim T (2014) Two distinct domains of Flo8 activator mediates its role in transcriptional activation and the physical interaction with Mss11. Biochemical and Biophysical Research Communications 449: 202-207. 
Klimes A, Dobinson KF (2006) A hydrophobin gene, VDH1, is involved in microsclerotial development and spore viability in the plant pathogen Verticillium dahliae. Fungal Genet Biol 43: 283-294.

Kobayashi O, Hayashi N, Kuroki R, Sone H (1998). Region of FLO1 proteins responsible for sugar recognition. J Bacteriol 180: 6503-6510.

Kobayashi O, Suda H, Ohtani T, Sone H (1996) Molecular cloning and analysis of the dominant flocculation gene FLO8 from Saccharomyces cerevisiae. Molecular \& General Genetics 251: 707-715.

Kolar M, Punt PJ, vandenHondel CAMJJ, Schwab H (1988) Transformation of Penicillium-Chrysogenum Using Dominant Selection Markers and Expression of an Escherichia-Coli Lacz Fusion Gene. Biological Chemistry Hoppe-Seyler 369: 852-852.

Lazo GR, Stein PA, Ludwig RA (1991) A DNA Transformation-Competent Arabidopsis Genomic Library in Agrobacterium. Bio-Technology 9: 963-967.

Lee YH, Dean RA (1993) cAMP Regulates Infection Structure Formation in the Plant Pathogenic Fungus Magnaporthe grisea. The Plant Cell 5: 693-700.

Li F, Palecek SP (2003) EAP1, a Candida albicans gene involved in binding human epithelial cells. Eukaryot Cell 2: 1266-1273.

Li Y, Que Y, Liu Y, Yue X, Meng X, Zhang Z, Wang Z (2015a) The putative Ggamma subunit gene MGG1 is required for conidiation, appressorium formation, mating and pathogenicity in Magnaporthe oryzae. Current Genetics 61: 641-651.

Li ZF, Liu YJ, Feng ZL, Feng HJ, Klosterman SJ, Zhou FF, Zhao LH, Shi YQ, Zhu HQ (2015b) VdCYC8, Encoding CYC8 Glucose Repression Mediator Protein, Is Required for Microsclerotia Formation and Full Virulence in Verticillium dahliae. PLoS One 10: e0144020.

Lin CJ, Sasse C, Gerke J, Valerius O, Irmer H, Frauendorf H, Heinekamp T, Straßburger M, Tran VT, Herzog B (2015) Transcription Factor SomA Is Required for Adhesion, Development and Virulence of the Human Pathogen Aspergillus fumigatus. PLoS Pathog 11: e1005205.

Lin JR, Hu J (2013) SeqNLS: nuclear localization signal prediction based on frequent pattern mining and linear motif scoring. PLoS One 8: e76864. 
Linder MB, Szilvay GR, Nakari-Setala T, Penttila ME (2005) Hydrophobins: the protein-amphiphiles of filamentous fungi. FEMS Microbiol Rev 29: 877-896.

Liu HP, Styles CA, Fink GR (1996) Saccharomyces cerevisiae S288C has a mutation in FL08 a gene required for filamentous growth. Genetics 144: 967-978.

Liu TB, Chen GQ, Min H, Lin FC (2009) MoFLP1, encoding a novel fungal fasciclinlike protein, is involved in conidiation and pathogenicity in Magnaporthe oryzae. J Zhejiang Univ Sci B 10: 434-444.

Lu J-P, Liu T-B, Lin F-C (2005) Identification of mature appressorium-enriched transcripts in Magnaporthe grisea, the rice blast fungus, using suppression subtractive hybridization. FEMS Microbiology Letters 245: 131-137.

Michaillat L, Mayer A (2013) Identification of genes affecting vacuole membrane fragmentation in Saccharomyces cerevisiae. PLoS One 8: e54160.

Min K, Son H, Lim JY, Choi GJ, Kim JC, Harris SD, Lee YW (2014) Transcription factor RFX1 is crucial for maintenance of genome integrity in Fusarium graminearum. Eukaryot Cell 13: 427-436.

Mittler T, Levy M, Chad F, Karen S (2010) MULTBLAST: A web application for multiple BLAST searches. Bioinformation 5: 224-226.

Moody RG, Williamson MP (2013) Structure and function of a bacterial Fasciclin I Domain Protein elucidates function of related cell adhesion proteins such as TGFBIp and periostin. FEBS Open Bio 3: 71-77.

Mulder N, Apweiler R (2007) InterPro and InterProScan: tools for protein sequence classification and comparison. Methods Mol Biol 396: 59-70.

Mumberg D, Muller R, Funk M (1994) Regulatable Promoters of SaccharomycesCerevisiae - Comparison of Transcriptional Activity and Their Use for Heterologous Expression. Nucleic Acids Research 22: 5767-5768.

Munro CA, Bates S, Buurman ET, Hughes HB, Maccallum DM, Bertram G, Atrih A, Ferguson MA, Bain JM, Brand A (2005) Mnt1p and Mnt2p of Candida albicans are partially redundant alpha-1,2-mannosyltransferases that participate in O-linked mannosylation and are required for adhesion and virulence. J Biol Chem 280: 1051-1060.

Naglik J, Albrecht A, Bader O, Hube B (2004) Candida albicans proteinases and host/pathogen interactions. Cell Microbiol 6: 915-926. 
Nahlik K, Dumkow M, Bayram O, Helmstaedt K, Busch S, Valerius O, Gerke J, Hoppert M, Schwier E, Opitz L (2010) The COP9 signalosome mediates transcriptional and metabolic response to hormones, oxidative stress protection and cell wall rearrangement during fungal development. Mol Microbiol 78: 964-979.

Neuhoff V, Arold N, Taube D, Ehrhardt W (1988) Improved Staining of Proteins in Polyacrylamide Gels Including Isoelectric-Focusing Gels with Clear Background at Nanogram Sensitivity Using Coomassie Brilliant Blue G-250 and R-250. Electrophoresis 9: 255-262.

Neuhoff V, Stamm R, Eibl H (1985) Clear Background and Highly Sensitive Protein Staining with Coomassie Blue Dyes in Polyacrylamide Gels - a Systematic Analysis. Electrophoresis 6: 427-448.

Neumann MJ, Dobinson KF (2003) Sequence tag analysis of gene expression during pathogenic growth and microsclerotia development in the vascular wilt pathogen Verticillium dahliae. Fungal Genet. Biol. 38: 54-62.

Octavio LM, Gedeon K, Maheshri N (2009) Epigenetic and Conventional Regulation Is Distributed among Activators of FLO11 Allowing Tuning of PopulationLevel Heterogeneity in Its Expression. PLoS Genetics 5: e1000673.

Odenbach D, Breth B, Thines E, Weber RW, Anke H, Foster AJ (2007) The transcription factor Con $7 p$ is a central regulator of infection-related morphogenesis in the rice blast fungus Magnaporthe grisea. Mol Microbiol 64: 293-307.

Padovan AC, Chaves GM, Colombo AL, Briones MR (2009) A novel allele of HWP1, isolated from a clinical strain of Candida albicans with defective hyphal growth and biofilm formation, has deletions of GIn/Pro and Ser/Thr repeats involved in cellular adhesion. Medical Mycology 47: 824-835.

Palmer GE, Cashmore A, Sturtevant J (2003) Candida albicans VPS11 is required for vacuole biogenesis and germ tube formation. Eukaryot Cell 2: 411-421.

Panozzo C, Cornillot E, Felenbok B (1998) The CreA repressor is the sole DNAbinding protein responsible for carbon catabolite repression of the alcA gene in Aspergillus nidulans via its binding to a couple of specific sites. J Biol Chem 273: 6367-6372. 
Pegg GF, Brady BL (2002) Verticillium Wilts. Wallingford N. Y.: CABI Publishing. doi: 10.1079/9780851995298.0000

Pham CL, Rey A, Lo V, Soules M, Ren Q, Meisl G, Knowles TP, Kwan AH, Sunde $M$ (2016) Self-assembly of MPG1, a hydrophobin protein from the rice blast fungus that forms functional amyloid coatings, occurs by a surface-driven mechanism. Sci Rep 6: 25288.

Phizicky EM, Fields S (1995) Protein-protein interactions: methods for detection and analysis. Microbiological Reviews 59: 94-123.

Pochanavanich P, Suntornsuk W (2002) Fungal chitosan production and its characterization. Lett Appl Microbiol 35: 17-21.

Prados-Rosales RC, Roldan-Rodriguez R, Serena C, Lopez-Berges MS, Guarro J, Martinez-del-Pozo A, di Pietro A (2012) A PR-1-like protein of Fusarium oxysporum functions in virulence on mammalian hosts. J Biol Chem 287: 21970-21979.

Rappsilber J, Mann M, Ishihama Y (2007) Protocol for micro-purification, enrichment, pre-fractionation and storage of peptides for proteomics using StageTips. Nat Protoc 2: 1896-1906.

Rauyaree P, Ospina-Giraldo MD, Kang S, Bhat RG, Subbarao KV, Grant SJ, Dobinson KF (2005) Mutations in VMK1, a mitogen-activated protein kinase gene, affect microsclerotia formation and pathogenicity in Verticillium dahliae. Current Genetics 48: 109-116.

Reinke J, Berthold G (1879) Die Zersetzung der Kartoffel durch Pilze. Untersuchungen aus dem Botanischen Laboratorium der Universität Göttingen. 1-100.

Rigden DJ, Mello LV, Galperin MY (2004) The PA14 domain, a conserved all-beta domain in bacterial toxins, enzymes, adhesins and signaling molecules. Trends in Biochemical Sciences 29: 335-339.

Roberts AN, Yanofsky C (1989) Genes expressed during conidiation in Neurospora crassa: characterization of con-8. Nucleic Acids Research 17(1): 197-214.

Ruepp A, Zollner A, Maier D, Albermann K, Hani J, Mokrejs M, Tetko I, Guldener U, Mannhaupt G, Munsterkotter M (2004) The FunCat, a functional annotation scheme for systematic classification of proteins from whole genomes. Nucleic Acids Res 32: 5539-5545. 
Rupp S, Summers E, Lo HJ, Madhani H, Fink G (1999) MAP kinase and cAMP filamentation signaling pathways converge on the unusually large promoter of the yeast FLO11 gene. Embo Journal 18: 1257-1269.

Sandini S, La Valle R, De Bernardis F, Macri C, Cassone A (2007) The 65 kDa mannoprotein gene of Candida albicans encodes a putative beta-glucanase adhesin required for hyphal morphogenesis and experimental pathogenicity. Cell Microbiol 9: 1223-1238.

Santhanam P, van Esse HP, Albert I, Faino L, Nurnberger T, Thomma BPHJ (2013) Evidence for Functional Diversification Within a Fungal NEP1-Like Protein Family. Molecular Plant-Microbe Interactions 26: 278-286.

Schinke J, Kolog Gulko M, Christmann M, Valerius O, Stumpf SK, Stirz M, Braus GH (2016) The DenA/DEN1 Interacting Phosphatase DipA Controls Septa Positioning and Phosphorylation-Dependent Stability of Cytoplasmatic DenA/DEN1 during Fungal Development. PLoS Genetics 12: e1005949.

Schnathorst WC (1982) The Relation of Verticillium dahliae Strains and Cotton Plantings to the Epidemic of Wilt Disease in Pistachio Nut Trees. Phytopathology 72: 960-960.

Sharkey LL, McNemar MD, Saporito-Irwin SM, Sypherd PS, Fonzi WA (1999) HWP1 functions in the morphological development of Candida albicans downstream of EFG1, TUP1, and RBF1. J Bacteriol 181: 5273-5279.

Shevchenko A, Wilm M, Vorm O, Mann M (1996) Mass spectrometric sequencing of proteins from silver stained polyacrylamide gels. Analytical Chemistry 68: 850-858.

Shi ZX, Leung H (1995) Genetic analysis of sporulation in Magnaporthe grisea by chemical and insertional mutagenesis. Molecular Plant-Microbe Interactions 8: 949-959.

Sievers F, Higgins DG (2014) Clustal Omega, accurate alignment of very large numbers of sequences. Methods Mol Biol 1079: 105-116.

Skamnioti P, Gurr SJ (2007) Magnaporthe grisea cutinase2 mediates appressorium differentiation and host penetration and is required for full virulence. Plant Cell 19: 2674-2689. 
Smith G (1948) The effect of adding trace elements to Czapek-Dox medium. Trans Br Mycol Soc 32: 280-283.

Son H, Kim MG, Min K, Seo YS, Lim JY, Choi GJ, Kim JC, Chae SK, Lee YW (2013) AbaA regulates conidiogenesis in the ascomycete fungus Fusarium graminearum. PLoS One 8: e72915.

Stanley CE, Stockli M, van Swaay D, Sabotic J, Kallio PT, Kunzler M, deMello AJ, Aebi M (2014). Probing bacterial-fungal interactions at the single cell level. Integrative Biology 6: 935-945.

Strauss J, Horvath HK, Abdallah BM, Kindermann J, Mach RL, Kubicek CP (1999) The function of CreA, the carbon catabolite repressor of Aspergillus nidulans, is regulated at the transcriptional and post-transcriptional level. Mol Microbiol 32: 169-178.

Studt L, Wiemann P, Kleigrewe K, Humpf HU, Tudzynski B (2012) Biosynthesis of fusarubins accounts for pigmentation of Fusarium fujikuroi perithecia. Appl Environ Microbiol 78: 4468-4480.

Sugiyama M, Nikawa J (2001) The Saccharomyces cerevisiae Isw2p-Itc1p complex represses INO1 expression and maintains cell morphology. Journal of Bacteriology 183: 4985-4993.

Sundstrom P (2002) Adhesion in Candida spp. Cell Microbiol 4(8): 461-469.

Tanabe S, Ishii-Minami N, Saitoh KI, Otake Y, Kaku H, Shibuya N, Nishizawa Y, Minami E (2011) The Role of Catalase-Peroxidase Secreted by Magnaporthe oryzae During Early Infection of Rice Cells. Molecular Plant-Microbe Interactions 24: 163-171.

Tao L, Yu JH (2011) AbaA and WetA govern distinct stages of Aspergillus fumigatus development. Microbiology-Sgm 157: 313-326.

Tian L, Wang Y, Yu J, Xiong D, Zhao H, Tian C (2016) The Mitogen-Activated Protein Kinase Kinase VdPbs2 of Verticillium dahliae Regulates Microsclerotia Formation, Stress Response, and Plant Infection. Front Microbiol 7: 1532.

Tian L, Xu J, Zhou L, Guo W (2014) VdMsb regulates virulence and microsclerotia production in the fungal plant pathogen Verticillium dahliae. Gene 550(2): 238-244. 
Timpel C, Zink S, Strahl-Bolsinger S, Schroppel K, Ernst J (2000) Morphogenesis, adhesive properties, and antifungal resistance depend on the Pmt6 protein mannosyltransferase in the fungal pathogen Candida albicans. J Bacteriol 182: 3063-3071.

Timpner C, Braus-Stromeyer SA, Tran VT, Braus GH (2013) The Cpc1 Regulator of the Cross-Pathway Control of Amino Acid Biosynthesis Is Required for Pathogenicity of the Vascular Pathogen Verticillium longisporum. Molecular Plant-Microbe Interactions 26: 1312-1324.

Tran VT, Braus-Stromeyer SA, Kusch H, Reusche M, Kaever A, Kuhn A, Valerius O, Landesfeind M, Asshauer K, Tech M (2014) Verticillium transcription activator of adhesion Vta2 suppresses microsclerotia formation and is required for systemic infection of plant roots. New Phytol 202: 565-581.

Tran VT, Braus-Stromeyer SA, Timpner C, Braus GH (2013) Molecular diagnosis to discriminate pathogen and apathogen species of the hybrid Verticillium longisporum on the oilseed crop Brassica napus. Appl Microbiol Biotechnol 97: 4467-4483.

Van Mulders SE, Christianen E, Saerens SMG, Daenen L, Verbelen PJ, Willaert R, Verstrepen KJ, Delvaux FR (2009) Phenotypic diversity of Flo protein familymediated adhesion in Saccharomyces cerevisiae. Fems Yeast Research 9: 178-190.

van Zutphen T, Todde V, de Boer R, Kreim M, Hofbauer HF, Wolinski H, Veenhuis M, van der Klei IJ, Kohlwein SD (2014) Lipid droplet autophagy in the yeast Saccharomyces cerevisiae. Mol Biol Cell 25: 290-301.

Verstrepen KJ, Derdelinckx G, Verachtert H, Delvaux FR (2003) Yeast flocculation: what brewers should know. Appl Microbiol Biotechnol 61: 197-205.

Verstrepen KJ, Klis FM (2006) Flocculation, adhesion and biofilm formation in yeasts. Molecular microbiology 60: 5-15.

Veses V, Richards A, Gow NA (2008) Vacuoles and fungal biology. Curr Opin Microbiol 11: 503-510.

Vitenshtein A, Charpak-Amikam Y, Yamin R, Bauman Y, Isaacson B, Stein N, Berhani O, Dassa L, Gamliel M, Gur C (2016) NK Cell Recognition of Candida glabrata through Binding of NKp46 and NCR1 to Fungal Ligands Epa1, Epa6, and Epa7. Cell Host Microbe 20: 527-534. 
Voegele RT, Hahn M, Lohaus G, Link T, Heiser I, Mendgen K (2005) Possible roles for mannitol and mannitol dehydrogenase in the biotrophic plant pathogen Uromyces fabae. Plant Physiol 137: 190-198.

Wang CS, St Leger RJ (2007) The MAD1 adhesin of Metarhizium anisopliae links adhesion with blastospore production and virulence to insects, and the MAD2 adhesin enables attachment to plants. Eukaryotic Cell 6: 808-816.

Wang X, Bai X, Chen DF, Chen FZ, Li BZ, Yuan YJ (2015) Increasing proline and myoinositol improves tolerance of Saccharomyces cerevisiae to the mixture of multiple lignocellulose-derived inhibitors. Biotechnology for Biofuels 8:142-155.

Wang Y, Tian L, Xiong D, Klosterman SJ, Xiao S, Tian C (2016) The mitogenactivated protein kinase gene, VdHog1, regulates osmotic stress response, microsclerotia formation and virulence in Verticillium dahliae. Fungal Genet Biol 88: 13-23.

Weber RW, Wakley GE, Thines E, Talbot NJ (2001) The vacuole as central element of the lytic system and sink for lipid droplets in maturing appressoria of Magnaporthe grisea. Protoplasma 216: 101-112.

Weber RWS (2002) Vacuoles and the fungal lifestyle. Mycologist 16(1): 10-20.

Wilhelm S (1955) Longevity of the Verticillium Wilt Fungus in the Laboratory and Field. Phytopathology 45: 180-181.

Wilson RA, Talbot NJ (2009) Under pressure: investigating the biology of plant infection by Magnaporthe oryzae. Nat Rev Microbiol 7: 185-195.

Xiao CL, Subbarao KV, Schulbach KF, Koike ST (1998) Effects of crop rotation and irrigation on Verticillium dahliae microsclerotia in soil and wilt in cauliflower. Phytopathology 88: 1046-1055.

Xiong D, Wang Y, Tang C, Fang Y, Zou J, Tian C (2015) VdCrz1 is involved in microsclerotia formation and required for full virulence in Verticillium dahliae. Fungal Genet Biol 82: 201-212.

Xiong D, Wang Y, Tian L, Tian C (2016) MADS-Box Transcription Factor VdMcm1 Regulates Conidiation, Microsclerotia Formation, Pathogenicity, and Secondary Metabolism of Verticillium dahliae. Front Microbiol 7: 1192. 
Xue C, Park G, Choi W, Zheng L, Dean RA, Xu JR (2002) Two novel fungal virulence genes specifically expressed in appressoria of the rice blast fungus. Plant Cell 14: 2107-2119.

Yan X, Li Y, Yue X, Wang C, Que Y, Kong D, Ma Z, Talbot NJ, Wang Z (2011) Two novel transcriptional regulators are essential for infection-related morphogenesis and pathogenicity of the rice blast fungus Magnaporthe oryzae. PLoS Pathog 7: e1002385.

Youseff BH, Holbrook ED, Smolnycki KA, Rappleye CA (2012) Extracellular superoxide dismutase protects Histoplasma yeast cells from host-derived oxidative stress. PLoS Pathog 8: e1002713.

Yu Y, Hube B, Kamper J, Meyer V, Krappmann S (2017) When green and red mycology meet: Impressions from an interdisciplinary forum on virulence mechanisms of phyto- and human-pathogenic fungi. Virulence: 1-10.

Zajac D, Karkowska-Kuleta J, Bochenska O, Rapala-Kozik M, Kozik A (2016) Interaction of human fibronectin with Candida glabrata epithelial adhesin 6 (Epa6). Acta Biochim Pol 63: 417-426.

Zeise K, von Tiedemann A (2001) Morphological and physiological differentiation among vegetative compatibility groups of Verticillium dahliae in relation to $V$. longisporum. J. Phytopathol. 149: 469-475.

Zhang Y, Zhao J, Fang W, Zhang J, Luo Z, Zhang M, Fan Y, Pei Y (2009) Mitogenactivated protein kinase hog1 in the entomopathogenic fungus Beauveria bassiana regulates environmental stress responses and virulence to insects. Appl Environ Microbiol 75: 3787-3795.

Zhao YL, Zhou TT, Guo HS (2016) Hyphopodium-Specific VdNoxB/VdPIs1Dependent ROS-Ca2+ Signaling Is Required for Plant Infection by Verticillium dahliae. PLoS Pathog 12: e1005793.

Zhou X, Zhang H, Li G, Shaw B, Xu JR (2012) The Cyclase-associated protein Cap1 is important for proper regulation of infection-related morphogenesis in Magnaporthe oryzae. PLoS Pathog 8: e1002911.

Zupancic ML, Frieman M, Smith D, Alvarez RA, Cummings RD, Cormack BP (2008) Glycan microarray analysis of Candida glabrata adhesin ligand specificity. Mol Microbiol 68: 547-559. 


\section{Abbreviations}

${ }^{\circ} \mathrm{C}$

$\Delta$

$\mathrm{Amp}^{\mathrm{R}}$

BLAST

bp

cAMP

CDM

cDNA

$\mathrm{cm}$

C-terminus

DMSO

DNA

DAPI

EDTA

Flo

GFP

GPI

g

h

$\mathrm{H}_{2} \mathrm{O}_{2}$

$\mathrm{HPH}$

$\mathrm{Kan}^{\mathrm{R}}$

$\mathrm{kb}$

$\mathrm{kDa}$

I

LB

LiAc

LCMS

$\mathrm{LisH}$

M

MAPK

mg degree Celsius

deletion

ampicillin resistance

basic local alignment search tool

base pair

cyclic adenosine monophosphate

Czapek-Dox medium

complementary DNA

centimeter

carboxyl terminus

dimethyl sulfoxide

deoxyribonucleic acid

4',6-diamidino-2-phenylindole, dilactate

ethylenediaminetetraacetic acid

flocculin

green fluorescent protein

glycosyl phosphatidylinositol

gram

hour

hydrogen peroxide

hygromycin phosphotransferase

kanamycin resistance

kilobase

kilo Dalton

liter

left border/ Luria Bertani medium

lithium acetate

liquid chromatography mass spectrometry

Lis homology

molar

mitogen-activated protein kinases

milli-gram 


\begin{tabular}{|c|c|}
\hline $\min$ & minute \\
\hline $\mathrm{ml}$ & milliliter \\
\hline$\mu g$ & micro-gram \\
\hline$\mu l$ & micro-liter \\
\hline $\mathrm{mM}$ & millimolar \\
\hline$\mu \mathrm{m}$ & micro-meter \\
\hline MM & minimal medium \\
\hline NAT & nourseothricin acetyltransferease \\
\hline NLS & nuclear localisation signal \\
\hline N-terminus & $\mathrm{NH}_{2}$ terminus \\
\hline OD & optical density \\
\hline ORF & open reading frame \\
\hline PBS & phosphate buffer saline \\
\hline PCR & polymerase chain reaction \\
\hline PDA & potato dextrose agar \\
\hline PDM & potato dextrose broth medium \\
\hline PEG & polyethylene glycol \\
\hline $\mathrm{RB}$ & right border \\
\hline RNA & ribonucleic acid \\
\hline rpm & revolutions per minute \\
\hline $\mathrm{S}$ & second \\
\hline SC-Ura & synthetic complete minus uracil medium \\
\hline SDS & sodium dodecyl sulphate \\
\hline SXM & simulated xylem medium \\
\hline TE & Tris- $\mathrm{HCl}$ and EDTA \\
\hline UV & ultraviolet \\
\hline Va & Verticillium albo-atrum \\
\hline$V d$ & Verticillium dahliae \\
\hline WT & wild-type \\
\hline YPD & yeast extract peptone dextrose \\
\hline
\end{tabular}




\section{List of Figures}

Figure 1. V. dahliae distribution 4

Figure 2. Conidia and microsclerotia of $V$. dahliae 6

Figure 3. Wilt disease symptoms of $V$. dahliae 7

Figure 4. V. dahliae life cycle 8

Figure 5. Expression of FLO11 is controlled by cAMP/PKA and MAPK $\begin{array}{ll}\text { pathways } & 11\end{array}$

Figure 6. Adhesion of $S$. cerevisiae on agar plates and in a liquid medium 12

Figure 7. Appressoria are required for the rice blast fungus $\begin{array}{ll}\text { Magnaporthe oryzae infection } & 15\end{array}$

Figure 8. NoxB and PIs1 are essential for hyphopodia peg formation 17

Figure 9. A FLO8 defective $S$. cerevisiae strain was used as a tool to screen for $\begin{array}{ll}\text { adhesion genes in Verticillium } & 18\end{array}$

Figure 10. Topology of the winged helix fold 19

Figure 11. The central developmental pathway of conidia formation in $\begin{array}{ll}\text { A. nidulans } & 21\end{array}$

Figure 12. Strategies of SOM1 deletion and confirmation in V. dahliae 34

Figure 13. Deletion and confirmation strategies of VTA3 gene in V. dahliae 35

Figure 14. Complementation of SOM1 and VTA3 in V. dahliae and confirmation strategies $\quad 36$

Figure 15. Gene locus and structure of SOM1 50

Figure 16. Gene locus and structure of VTA3 51

Figure 17. Som1 and Vta3 are nuclear proteins 52

Figure 18. SOM1 and VTA3 can reprogram non-adhesive FLO8-deficient

$S$. cerevisiae strains to adhesion on agar plates

Figure 19. Som1 and Vta3 can activate flocculation of FLO8-defective

S. cerevisiae in liquid medium

Figure 20. Som1 promotes the expression of FLO1 and FLO11 in FLO8-deficient $S$. cerevisiae

Figure 21. Vta3 stimulates the expression of FLO1 and FLO11 in FLO8-defective S. cerevisiae

Figure 22. Confirmation of deletion and complementation strains of 
Figure 23. Confirmation of deletion and complementation strain of VTA3 in V. dahliae 58

Figure 24. Som 1 is required for $V$. dahliae hyphal clumping 59

Figure 25. Som 1 suppresses $V$. dahliae biomass formation 60

Figure 26. Som 1 is necessary for adhesion of $V$. dahliae on polystyrene plates 61

Figure 27. Som 1 is necessary for $V$. dahliae adhesion to GelBond film 62

Figure 28. Som 1 and $V t a 3$ are required for introduction of disease symptoms in tomatoes

Figure 29. V. dahliae Som 1 and Vta3 are essential for plant infection

Figure 30. Fungal Som1 and Vta3 are sequentially required for root penetration and root colonisation

Figure 31. Som1 and Vta3 are required for successful root colonisation 67

Figure 32. Som 1 and Vta3 promote conidia formation 68

Figure 33. Som 1 and Vta3 control microsclerotia formation 70

Figure 34. The overexpression of SOM1 enhances the number of microsclerotia

Figure 35. Som1 and Vta3 antagonise in oxidative stress response 72

Figure 36. Som1 and Vta3 affect growth on different carbon sources 74

Figure 37. Som 1 is necessary for aerial hyphae formation 75

Figure 38. Som1 is required for aerial hyphae and hyphal branching of V. dahliae 76

Figure 39. Som1 is required for septa positioning in $V$. dahliae 77

Figure 40. $V$. dahliae Som1 is essential for normal vacuole size 78

Figure 41. Som 1 and Vta3 control the expression of VTA genes 79

Figure 42. Functional categorisation analyses of twenty proteins significantly down regulated in the SOM1 deletion strain

Figure 43. Som 1 controls the expression of putative adhesion target genes

Figure 44. Som 1 and Vta3 control the expression of putative target genes of conidia and microsclerotia formation, oxidative stress response, and virulence

Figure 45. AfSomA can partly rescue the growth of the SOM1 deletion strain

Figure 46. AfSomA can restore the conidia and microsclerotia formation in the SOM1 deletion strain of $V$. dahliae

Figure 47. Som1 might directly bind to the promoter of FLO11

Figure 48. Vta3 activate FLO11 via repressing the expression of SFL1 
Figure 49. Model of Som1 and Vta3 control fungal development and virulence by regulation of VTA genes

Figure 50. Model of Som1 and Vta3 control of conidia formation 97

Figure 51. Model of Som1 reregulates the oxidative stress response 100

Figure 52. Model of Vta3 repression of the oxidative stress response via $\begin{array}{ll}\text { suppressing INO1 } & 101\end{array}$

Figure 53. Model of functions of Som1 and Vta3 in V. dahliae 104 


\section{List of Tables}

Table 1. Primers used in this study $\quad 24$

$\begin{array}{ll}\text { Table 2. Plasmids used in this study } & 29\end{array}$

Table 3. Fungal strains used in this study 31

Table 4. List of abundant proteins significantly changed in the SOM1 deletion strain 81

Table 5. Som 1 putative interaction partners identified by GFP-trap enrichment 85

Table 6. Vta3 putative interaction partners identified by GFP-trap enrichment 86 


\section{Acknowledgements}

Firstly, I would like to express my sincere gratitude to my main supervisor, Prof. Dr. Gerhard H. Braus for giving me the opportunity to do my PhD training in his laboratory and his kind support, helpful pieces of advice and continuous inspiration under his supervisorship.

I would also like to thank my supervisor Dr. Susanna Braus-Stromeyer for the good guidance both in science and general life situations in Göttingen. Without her recommendation, my family could not have suitably reunited with me here in Germany.

I wish to thank Prof. Dr. Stefanie Pöggeler for being on my thesis committee and her insightful suggesion in all progress reports and thesis committee meetings.

My appreciation also goes to Prof. Dr. Ivo Feussner, Prof. Dr. Kai Haimel, PD Dr. Michael Hoppert, and Prof. Dr. Rolf Daniel for being members of my examination board.

I am very thankful to Dr. Rabea Schlüter from the Imaging Center of the Department of Biology, University of Greifswald for scanning electron microscopy. I also want to thank Dr. Claire E. Stanley from Plant-Soil Interactions, Agroecology and Environment Research Division, Agroscope, Switzerland for providing microfluidic devices which were used for hyphal branching experiments.

I would like to thank Dr. Rebekka Harting, Dr. Mirit Kolog Gulko, Dr. Razieh Karimi, Dr. Alexandra Kleinknecht, Godwin Sokpor, Benedict Dirnberger and Annalena Höfer for proofreading and correcting this thesis. Similarly, I want to thank other members of the department such as Dr. Oliver Valerius, Dr. Blaga Popova, Dr. VanTuan Tran, Dr. Alinne Ambrósio, Dr. Kerstin Schmitt, and Kai Nesemann who helped me during my doctoral studies.

I thank all the members of my department for providing the friendly working environment, discussing, and giving excellent advice for my experiments.

My appreciation also goes to Heidi Northemann and Nicole Scheiter for official documents and chemical supply, Maria Meyer for technical support in yeast genetics, Andrea Wäge and Gaby Heinrich for preparing buffers. 
I wish to thank Prof. Dr. Xuan-Binh Ngo who supported my bachelor and master thesis. He always encouraged me to going abroad for PhD training.

Special thanks to my parents and sisters for their endless support in my daily living and also taking care of my son during the time my wife and I were not in Vietnam.

Finally, I would like to thank my wife and son for their unique encouragement and support in my life. Without them, this valuable piece of work would not have been accomplished. 


\title{
Curriculum vitae
}

\author{
Personal information \\ Tri-Thuc Bui \\ Born on May 29th 1984 \\ In Thai Nguyen, Vietnam
}

\section{Education}

1990 - 1999 Elementary and junior education in Thai Nguyen, Vietnam

2000 - 2002 High school education in Thai Nguyen, Vietnam

\section{Scientific background}

2003 - 2007 Bachelor of Science at Thai Nguyen University of Agriculture and Forestry, Thai Nguyen, Vietnam

2007 - 2008 Assistant researcher at Agricultural Genetics Institute

2008 - 2013 Assistant researcher and assistant lecturer at Thai Nguyen University of Agriculture and Forestry, Thai Nguyen, Vietnam

2009 - 2012 Master of Science in Experimental Biotechnology at Thai Nguyen University, Thai Nguyen, Vietnam

2013 - 2017 Scientific assistant and PhD student in the lab of Prof. Dr. Gerhard Braus at Department of Molecular Microbiology and Genetics, Institute of Microbiology and Genetics, Georg-August University Göttingen 